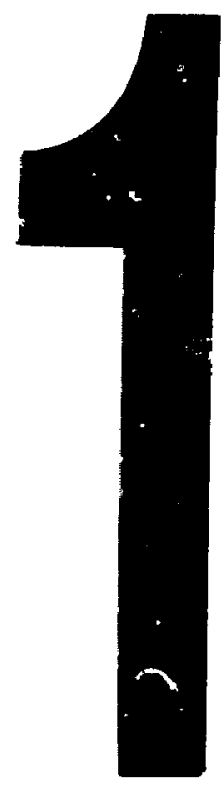

PM-1 31/2" $\times$ 4" PHOTOGRAPHIC MICROCOPY TARGET : NBS 1010a ANSI/ISO \#2 EOLIV'ALENT

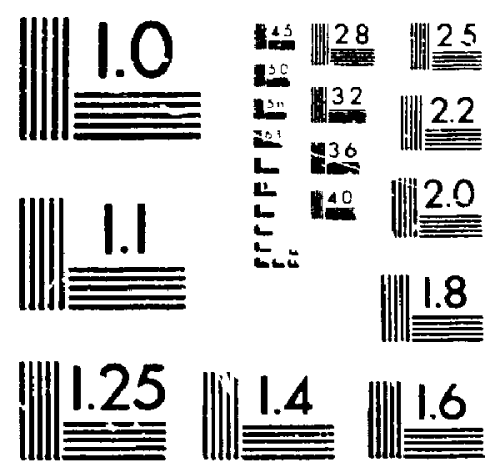


National Library

of Canada

Canadian Theses Service

Ottawa, Canada

KIA ON4
Biblıothèque nationaie du Canada

Service des thèses canadiennes

\section{NOTICE}

The quality of this microform is heavily dependent upon the quality of the original thesis submitted for microfilming. Every effort has been made to ensure the highest quality 0 . reproduction possible.

If pages are missing, contact the university which granted the degree.

Some pages may have indistinct print especially it the original pages were typed with a poor typewriter ribbon or if the university sent us an inferior photocopy.

Heproduction in full or in part of this microform is governed by the Canadian Copyris! It Act. R.S.C. 1970, C. C-30, and subsequent amendments.

\begin{abstract}
AVIS
La qual'té de celte microlorme dépend grandement de la qualité de la thèse soumıse au micrulilmage Nous avons tout fait pour assurer une qualité supérieure de reproduc. tion.

Sil manque des pages, veuillez communiquer avec l'université qui a contéré le grade.

La qualité d'ımpressıon de certaınes pages peul laisser à désırer, surtout sı les pages orıginales ont été dactylographiées à l'aide d'un ruban usé ou si l'université nous a fatt parvenir une photocopie de qualité intérieure

La reproduction, méme partielle, de celte m.croforme est soumise a la Loi canadienne sur le drolt dauteur. SRC 1970. c. C-30, et ses amendements subséquents
\end{abstract}




\title{
DEVELOPMENT OF A COMPUTATIONAL MODEL FOR THE LM-1600 INDUSTRIAL GAS TURBINE
}

\author{
by \\ Ping Zhu \\ B.Eng., Eastern China Petroleum In., 1982
}

A thesis submitted to the Faculty of Graduate

Studies and Research in partial fulfillment of the requirements for the Degree of

Master of Engineering

\author{
Department of \\ Mechanical and Aerospace Engineering \\ Carleton University \\ Ottawa, Ontario \\ Canada
}

February, 1991 
The author has granted an irrevocable monexclusive licence allowing the National Library of Canada to reproduce, loan, distribute or sell copies of his/her thesis by any means and in any form or format, making this thesis available to interesied persons.

The author retains ownership of the copyright in his/her thesis. Neither the thesis nor substantial extracts from it may be printed or otherwise reproduced without his/her permission.
L'auteur a accordé une licence irrévocable et non exclusive permettant à la Bibliothèque nationale du Canada de reproduire, préter. distribuer ou vendre des copies de sa these de quelque manière et sous quelque forme que ce soit pour mettre des exemplaires de cette thèse à la disposition des personnes intéressées.

L'auteur conserve la proprièté du droit d'auteur qui protège sa thèse. Ni ta thèse ni des extraits substantiels de celle-ci ne doivent être imprimés ou autrement reproduits sans son autorisation. 


\section{Carleton University}

Ottawa, Canada K1S5B6

Thesis contains black \& white photographs \&/or explanatory tables which when microfilmed may lose their significance. The hardcopy of the thesis is available upon request from Carleton Unxversity Lubrary. 


\section{Acceptance Sheet}

The undersigned recommend to the Faculty of Graduate Studies and Kesearch, acceptance of the thesis: Development of a Computational Model for The LM-1600 Industrial Gas Turbine, submitted by Ping Zhu, in partial fulfillment of the Degree of Master of Engineering.
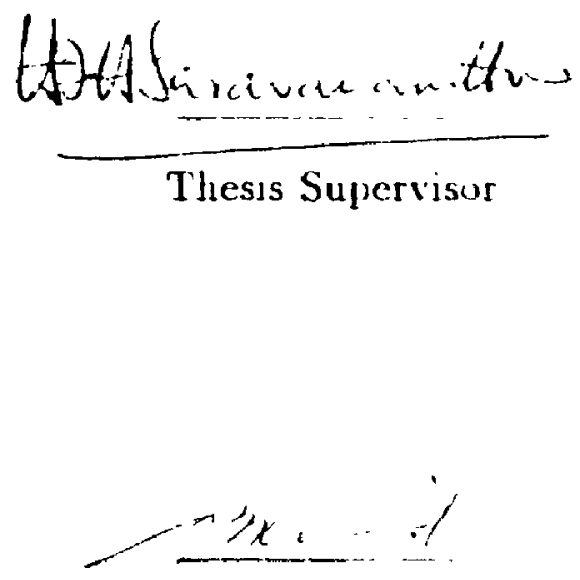

Chairman

Department of Mechanical

\& Aerospace Engıneering 


\section{Abstract}

The LM-1600, an advanced twin-spool industrial gas turbine, is being used in the gas pipeline system of NOVA Corporation of Alberta to function as pipeline compressor divivers. As part of an Engine Health Monitoring system, a computer model of the LM-1600 has been develoyed to simulate the performance of the engine.

The thermodynamic model is based on engine component matching calculations, using two different types of component model. The first one is based on a generalized turbine model, which is adequate for overall performance calculation; the second one uses a generalized compressor characteristic, giving the user considerably more insight into the engine behaviour.

A new solution method of the component matching has been developed during this work. In order to avoid unnecessary iteration and graphic calculation as well as the solving of a nonlinear equation set, a hot end solution procedure and a one-dimensional seaiching technique are combined to derive a Hot End Method. Using this melhod, the pre-assumption of component working condition is not necessary, and coupling the model with the existing field environment is facilitated.

Comparison between model predictions and field measurements has shown good agreement. The model is designed to be readily usable by an engine operator using the normal station PC computer. 


\section{Dedication}

To my Parents 


\section{Acknowledgements}

The woik introduced in this thesis was done by the author while studying at the Department of Mechanical and Aercspacc Eng neering of Carleton University, Ottawa. I would like to use this opportunity to express my deep gratitude to all my teachers in the department for their teaching and patient guidance during the course of my graduate study. Specially I would like to thank Prof. S.A. Sjolander for his initial help when I started this program.

I would like to thank all my friends and classmates in the department. Their friendship and help are always the source of encouragement.

I would also like to thank NOVA Corporation of Alberta for providing their field measurement data.

Finally I very sincerely thank my thesis supervisor, Prof. H.I.H. Saravanamuttoo, for his support, his valuable supervising and his help. His working attitude always encourages me towards the best. 


\section{CONTENTS}

Abstract iii

Acknowledgements v

Contents vi

List of Tateles $\quad x$

List of Figures

Nomenclaîure $\quad x v$

Chapter 1. Introduction 1

1.1 General 1

1.2 Thesis Objective $\quad 2$

1.3 Brief Review of Previous Work 3

Chapter 2. The LM-1600 6

2.1 Components 6

2.2 Cooling Air Flow Pattern 8

2.3 Variable Bleed Valve $\quad 8$

Chapter 3. Design Point Calculation 9

3.1 Basic Assumptions 10

3.2 General 10

3.2.1 Concepts of Design Point Calculation 10

3.2.2 Known Design Point Parameters 11 
3.2.3 PL. pose of DPC for the Model of the LM-1600 12

3.3 The DPC Model 13

3.4 Solution of The DPC Model 16

3.4.1 Model Analysis 16

3.4.2 Solution Procedures $\quad 17$

3.4.3 Validating Analysis 18

3.5 Conclusions $\quad 22$

Chapter 4. Hot End Method 23

4.1 Requirements for Engine Modelling 23

4.2 Mathematic Model and Component Matching Process 24

4.2.1 Governing Equations and

Component Matching Calculation 24

4.2.2 Governing Equation Analysis 27

4.3 Hot End Solution Process 29

4.3.1 Generalized Turbine Model 29

4.3.2 Power Turbine Pressure Ratio, $P_{6} / P_{7} \quad 31$

4.3.3 Initrate the Solution Process $\quad 32$

4.3.4 Solution Procedure 33

4.4 One-Dimensional Searching 34

4.4.1 Relationships between $P_{6} / P_{7}$, and Other Parameters 34

4.4.2 One-Dimensional Searchıng Technique 36

4.5 Overview of Hot End Method 37

Chapter 5. The LM-1600 Model and Program Analysis 41

5.1 Mathematic Model of The LM-1600 42 
5.1.1 Flow Compatibility 42

5.1.2 Governing Equations 42

5.1.3 Generalized Turbine Model 44

5.2 Component Models $\quad 45$

5.2.1 Blow off Air Flow Modelling 45

5.2.2 Fuel Flow and Combustion Process Modelling 46

5.2.3 Cooling Air Flow Modelling 47

5.2.4 Consideration of Inlet Guide Vanes and Variable Stator Vanes 47

5.3 Compressor Modelling 48

5.3.1 The Generalized Compressor Characteristic $\quad 48$

5.3.2 Esiimation of Compressor Efficiencies $\quad 49$

5.4 Program Structure 51

5.4.1 Overall Calculation Flow Path 51

5.4.2 The Main Calculation 52

5.5 Convergence Analysis $\quad 53$

5.5.1 Iterating Convergence $\quad 53$

5.5.2 One-Dimensional Searching Convergence $\quad 54$

5.6 Comparison with Field Measurements 55

Chapter 6. The Model Applications 57

6.1 DPC and Engine Modification 57

6.2 Selection of The Fixed Parameter 60

6.3 Specification of Runn.ng Conditions 60

6.3.1 Effect of Inlet Conditions $\quad 60$

6.3.2 Deteriorated Engine Conditions $\quad 66$

6.4 The Model Outputs $\quad 70$ 
6.4.1 Table Outputs $\quad 70$

6.4.2 Mapping Calculation $\quad 74$

6.5 Coupling with Field Operation $\quad 75$

6.5.1 Fault Matrices $\quad 75$

6.5.2 Fault Detection $\quad 78$

6.5.3 Other Functions $\quad 79$

6.6 Hardware Requirement $\quad 80$

Chapter 7. Recommendations for Further Work and Conclusions 81

7.1 Research Of Component Mathematic Models 81

7.2 Conclusions $\quad 82$

$\begin{array}{ll}\text { References } & 84\end{array}$

$\begin{array}{ll}\text { Appendix: Computer Program } & 87\end{array}$ 


\section{List of Tables}

Table 1. Effect of Compressor Polytropic Efficiency on Cooling Air Flow 19

Table 2. Analysis of Cooling Air Distribution 20

Table 3. Analysis of DPC Results 21

Table 4. Comparison of Different Turbine Simulation Methods 31

Table 5. Relationships between $\frac{P_{6}}{P_{70}}$ and Other Parameters 35

Table 6. Table Output 1: Design Point Calculation 58

Table 7. Selection of The Fixed Parameter and Valid Range 59

Table 8. Effect of Inlet Temperature $\left(H_{\text {out }}=\right.$ Constant $)$

Table 9. Effect of Inlet Temperature

(LP-spool speed $=$ Constant $) \quad 62$

Table 10. Effect of Inlei Pressure $\left(H_{\text {out }}=\right.$ Constant $)$

Table 11. Effect of Inlet Pressure (LP-spool speed = Constant) 64

Tabie 12. Effect of Deteriorated Engine

$\left(W_{\text {out }}=\right.$ Independent Variable $)$

Tabje 13. Effect of Deteriorated Engine (LP-spool Speed $=$ Independent Variable) $\quad 69$

Table 14. Table Uutput 2: Main Calculation Results 71

Table 15. Table Output 3: Main Calculation Results (continue) 72

Table 16. Fault Matrix ( $W_{\text {out }}=$ Independent Variable) $\quad 76$

Table 17. Fault Matrix (LP-speed $=$ Independent Variable) $\quad 77$ 


\section{List of Figures}

Figure 1. The LM-1600

Figure 2. The General Flow Pattern and Station Numbering System 111

Figure 3. Flow Pattern at Design Point 112

Figure 4. Cooling Air Flow Rate vs Polytropic Efficiency 113

Figure 5. Trends of Cooling Air Flow Rates vs $T_{5}$

$\left(\eta_{c \infty}=0.88\right)$

Figure 6. Trends of Cooling Air Flow Rates vs $T_{5}$

$\left(\eta_{c \infty}=0.90\right)$

Figure 7. Trends of Cooling Air Flow Rates vs $T_{5}$ $\left(\eta_{c \infty}=0.92\right)$

Figure 8. Eligible $T_{5}$ Region $\left(\eta_{c \infty}=0.88\right)$

Figure 9. Eligible $T_{5}$ Region $\left(\eta_{c \infty}=0.90\right)$

Figure 10. Eligible $T_{5}$ Region $\left(\eta_{c \infty}=0.92\right)$

Figure 11. Twin-spool Land Based Gas Generator and Station Numbering System

Figure 12. Component Characteristics

Figure 13. Relation of Component Matching Governing Equations and Unknowns

Figure 14. Modified Equation and Unknown Relation

Figure 15. Mathematic Model of Twin-spool Gas Turbine

Figure 16. Relaticrships between $P_{6} / P_{78}$ and Other Parameters

Figure 17. LPC Characteristic of LM-1600 
and Comparison of Operating Lines

Figure 18. HPC Characteristic of LM-1600

and Comparison of Operating Lines

Figure 19. Compressor Efficiency Analysis (1':

LPC Operating Region ( $\eta_{l p c}=\eta_{l p c-\text { deatgn }}$ )

Figure 20. Compressor Efficiency Analysis (2):

HPC Operating Region ( $\eta_{l p c}=\eta_{l p c-\text { deagn }}$ )

Figure 21. Compressor Efficiency Analysis (3):

LPC Operating Region ( $\eta_{h p c}=\eta_{h p c-\text { design }}$ )

Figure 22. Compressor Efficiency Analysis (4):

HPC Operating Region ( $\eta_{h p c}=\eta_{h p c-d e s g n}$ )

1311

Figure 23. Overall Program Structure of LM16

Figure 24. Flow Path of The Main Calculation of LM16

Figure 25. Comparison between Model Prediction and

Field Measurement ( $N_{l}$ vs $\|_{\text {out }}$ )

134

Figure 26. Comparson between Model Prediction and

Field Measurement ( $N_{h}$ vs $H_{\text {out }}^{\circ}$ )

Figure 27. Comparison between Model Prediction and

Field Measurement $\left(N_{l}\right.$ vs $\left.N_{h}\right)$

Figure 28. Comparison between Model Prediction and

Field Measurement $\left(P_{3 \text { static }} / P_{1}\right.$ vs $\left.N_{h}\right)$

Figure 29. Comparison between Model Prediction and

Field Measurement (EGT vs $N_{h}$ )

Figure 30. Comparison of SFC

Figure 31. Effect of Inlet Conditions on LPC Running Line

Figure 32. Effect of Inlet Conditions on HPC Running Line

Figure 33. LPC Mechanical Damage 


$$
\text { ( } \left.W_{\text {out }}=\text { independent variable }\right)
$$

Figure 34. Compressur Excessive Leakage

$$
\text { ( } W_{\text {out }}=\text { independent variable) }
$$

Figure 35. HPT Mechanical Damage

$$
\text { ( } W_{\text {out }}=\text { independent variable) }
$$

Figure 36. HPT Erosion ( $W_{\text {out }}=$ independent variable)

Figure 37. LPT Erosion ( $W_{\text {out }}=$ independent variable)

Figure 38. LPC Mechanical Damage

$$
\text { (LP-spool speed }=\text { independent variable) }
$$

Figure 39. Compressor Excessive Leakage

$$
\text { (LP-spool speed }=\text { independent variable) }
$$

Figure 40. HPT Mechanical Damage

$$
\text { (LP-spool speed }=\text { independent variable) }
$$

Figure 41. HPT Erosion

Figure 42. LPT Erosion

$$
\text { (LP-spool speed }=\text { independent variable) }
$$

Figure 4:̈. Effect of Deteriorated Engine

on LPC Running Line

Figure 44. Effect of Deteriorated Engine

on HPC Running Line

Figure 45. Graphic Output 1, LPC Running Line

Figure 46. Graphic Output 2, HPC Running Line

Figure 47. Graphic Output 3, LP-spool Mechanical Speed vs. HP-spool Mechanical Speed

Figure 48. Graphic Output 4, LP-spool Mechanical Speed vs. LPC Pressure Ratio 
Figure 49. Graphic Output 5, HP-spool Mechanical Speed vs. HPC Pressure Ratio

Figure 50. Graphic Output 6, OPR (static) vs. HP-spool Mechanical Speed

Figure 51. Graphic Output 7, HP-spool Mechanical Speed vs. Work Output

Figure 52. Graphic Output 8, LP-spool Mechanical Speed vs. Work Output

Figure 53. Graphic Output 9, Compressor Efficiencies vs. Work Output

Figure 54. Graphic Output 10, Gas Generator EGT vs. HP-spool Mechanical Speed

Figure 55. Graphic Output 11, Mass Flow Ratio, $\frac{M_{2}}{M_{1}}$, vs. LP-spool Mechanical Speed

Figure 56. Graphic Output 12, SFC vs. Work Output 


\section{Nomenclature}

$$
\begin{aligned}
& \text { A - flow area } \\
& C_{p}-\text { specific heat } \\
& \mathrm{f} \text { - fuel / air ratio } \\
& \mathrm{h}-\text { enthalpy } \\
& \mathrm{M} \text { - mass flow } \\
& \mathrm{m}-\text { cooling air flow } \\
& \mathrm{P}-\text { pressure } \\
& \mathrm{R}-\text { gas constant } \\
& \mathrm{T}-\text { temperature } \\
& \mathrm{W}-\text { work } \\
& \text { Wout - work output } \\
& \eta-\text { efficiency } \\
& \gamma-\text { opecific heat ratio } \\
& \epsilon_{\boldsymbol{a}}=\left(\frac{\gamma-1}{\gamma}\right) \text { air } \\
& \epsilon_{g}=\left(\frac{\gamma-1}{\gamma}\right)_{g a s} \\
& \frac{M \sqrt{T}}{P} \text { (or } \mathrm{Q} " \text { in graphics) - "non-dimensional mass flow" } \\
& \text { (for short: non-d mass flow.) }
\end{aligned}
$$




\section{Subscripts}

$$
\begin{aligned}
& \mathrm{a} \text { - air } \\
& \mathrm{b}-\text { blown off } \\
& \mathrm{c} \text { - compressor } \\
& \mathrm{f} \text { - fuel } \\
& \mathrm{g} \text { - gas } \\
& \mathrm{m}-\text { mechanical } \\
& \mathrm{s} \text { - static } \\
& \mathrm{t} \text { - turbine } \\
& \text { therm - thermal } \\
& \infty-\text { polytropic }
\end{aligned}
$$

\section{Abbreviations}

$$
\begin{aligned}
& \text { DPC - Design Point Calculation } \\
& \text { ECV - Effective Calorific Value } \\
& \text { EGT - Exhaust Gas Temperature } \\
& \text { EHM - Engine Health Monitoring } \\
& \text { GPA - Gas Path Analysis } \\
& \text { HPC - High Pressure Compressor } \\
& \text { HPT - High Pressure Turbine } \\
& \text { IGV - Inlet Guide Vanes } \\
& \text { IGHP - Isentropic Gas Horsepower } \\
& \text { ISO - International Standard Organization } \\
& \text { LPC - Low Pressure Compressor } \\
& \text { LPT - Low Pressure Turbine } \\
& \text { OPR - Overall Pressure Ratio }
\end{aligned}
$$




$$
\begin{aligned}
& \text { PR - Pressure Ratio } \\
& \text { TIT - Turbine Inlet Temperature } \\
& \text { VBV - Variable Bleed Vaive } \\
& \text { VSV - Variable Stator Vanes } \\
& \text { sfc - specific fuel consumption }
\end{aligned}
$$

\section{Station Numbers}

$$
\begin{aligned}
& 1 \text { - LPC inlet } \\
& 2 \text { - HPC inlet } \\
& 23 \text { - Exit from the } 4^{\text {th }} \text { stage of the HPC } \\
& 3 \text { - Exit from HPC } \\
& 4-\text { HPT inlet } \\
& 45 \text { - Gap between HPT stator and rotor } \\
& 5 \text { - LPT inlet } \\
& 6 \text { - Power Turbine inlet } \\
& 7 \text { - Exic from Power Turbine }
\end{aligned}
$$




\section{Chapter 1}

\section{INTRODUCTION}

\subsection{General}

As the prices of mechanical manufacturing and fuel grow, the importance of gas turbine modelling and performance monitoring has been realized by more and more gas turbine engine designers and users. The rapid development of computational technique makes this possible.

In the gas turbine design field, the significance of engine modelling is that it can generate engine performance information at the preliminary design stage. This helps the designer physically foreseeing his design before committing to actual hardware. An engine model based on detailed component models can also help the designer to make suitable adjustments of the design to obtain a better engine with lower cost. In order to make a competitive engine, the designer should have a good understanding of competing engines; engine modelling is a powerful tool at the disposal of the designer.

In the field of gas turbine operations, an Engine Health Monitoring (EHM) system based on instrumentation, operating parameter analysis, thermodynamic analysis and component modelling indicates the working status of a gas turbine. This not only gives the engine operator sufficient information to find any deteriorated or abnormal engine running condition before it grows to a real disaster, but als', provides some physical insight of the gas turbine to the user, adding to his pool of 
knowledge. The latter is especially important when a new model of gas turbine is initially introduced.

Engine modelling and performance monitoring also have economic benefits. From the limited natural resources in the world and the unlimited use of energy, the tendency for rising fuel prices is inevitable. The cost ratio of fuel to maintenance has reached the level which indicates that, regardless of the mechanical health of a gas turbine, an early overhaul for fuel saving reasons, according to certain engine deteriorated conditions, is becoming necessary. Performance simulation and monitoring can provide good information on engine condition to the overhaul scheduler.

The meaning of engine modelling and performance monitoring is usually more inan engine protection itself. It is well known that an aircraft engine failure could cause loss of life. It might also be known that industrial gas turbine failure could also cause severe problems. Examples of this could be the gas turbines used for electrical power generation and energy transportation; any failures of these engines would weaken the existing operating and utility circle. The resulting economic losses could be very high. All these losses can be largely protectea by engine monitoring and control systems.

\subsection{Thesis Objective}

The aim of ihis thesis is to develop a computational model of an advanced industrial gas turbine, the LM-1600, recently introduced by General Electric, and ordered by a number of pipeline companies. Among the users, NOVA Corporation of Alberta, an Alberta based natural gas shipping company, has used the engine as pipeline compressor drivers in its natural gas pipeline system, and provided some field measurement data obtained by the MHEALTH health monitoring system.

Functioning as the engine performance simulator, the model would be a part of the Engine Health Monitoring (EHM) system, and could also be independently used by the field operators for the purposes of operating practice, performance prediction and on-site performance analysis. To be useful in the field, the following requirements should be met:

1) It should be able to generate all the necessary engire Iunning information 
to help field operators judge the engine working conditions and determine their operating strategies.

2) The use of this model should not cause any significant increase of field instruments and equipment, as well as operator work load. Since Personal Computers have been installed at all pipeline compressor siations, this model should be a portable PC model.

3) The model should be user friendly, i.e. the operation of the model should be in some sense similar to the operation of the real engine, and users do not need special training.

4) Although the field users, pipeline operators, are experts on natural gas transportation, they would not be experts on gas turbines. The model should not only be a mathematical representation of the real engine, but also help the users to understand the physical meaning of the results and gain some operating experience.

5) The model should be able to simulate certain deteriorated engine conditions to create fault matrices and help the users to predict and detect faults.

\subsection{Brief Review of Previous Work}

From the thermodynamic point of view, the performance of a gas turbine is the combination of several continuous thermodynamic processes of the working flow Theprocesses obey the laws of thermodynamics and can be mathematically expressed; component efficiencies can be introduced to represent the difference betwecn real processes and ideal ones.

If the thermodynamic parameters of the working flow at inlet and outlet of each component are measured, the process can be evaluated by using the laws of thermo. dynamics and the definition of the component efficiency. A large drop of efficiency usually means that severe deterioration or mechanical damage of the component has occured, and mechanical overhaul or changing the component is then required

One example of perfurmance monitoring systems based on this consideration is Gas Path Analysis (GPA). The concept of GPA on gas turbine engines was intro. duced by Urban (1973). Development and the application to pipeline gas turbunes had been introduced by Saravanamuttoo (1974) and has been widely used since 
then. By this method, some important un-measured parameters, such as Turbine Inlet Temperature (TIT), Inlet Mass Flow and Component Efficiencies, can be deduced from the measured parameters by using the conservation of mass and energy. Comparing the present engine running information with that of a normal engine, the degree of deterioration can be found. The problem investigation could be detailed to the component level. The GPA introduced by Saravanamut too does not require new equipment and instruments, has only minimum computational work and the concept is valid for any configuration of gas turbine, so it is potentially a powerful tcol for performance monitoring.

In an EHM system, instrumentation faults should be separated from engine faults. Otherwise, the instrument errors appearing in measurement will be interpreted as the errors of performance, hence engine deterioration.

Agrawal, Maclsaac and Saravanamuttoo (1979) reconmended a data validation package for on-site performance measurements of gas turbines. Basically it compares the measured and GPA calculated parameters with the pre-established baseline data of a normal engine, checks the deviations of so called Key Engine Variables and determines whether the deviations are caused by the instrumentation or the engine faults. Tolerable engine deterioration can be traced by shifting the baseline according to the newly measured value of fuel flow rate.

From the pipeline user's point of view, Williams (1981) made some modifications to GPA and reduced the number of faults to be examined to a list of Gas Turbine Modelling Parameters. Similar to the baseline setting in GPA and Design Point Calculation introduced later, it first calculates the Gas Turbine Modelling Parameters by using engine testing parameters after each overhanl. Then the measured operating pararneters can be processed by simple component matching with certain assumptions based on the consideration of the limited operating range of gas pipeline compressors. The results are compared with the Gas Turbine Modelling Parameters and the separation of engine faults and instrument faults can be done by a routine analysis procedure. A good background of experience in testing engines in good condition is essential for successiul fault analysis.

The work described above mainly focus on the thermodynamic processes in gas turbine. Components of the engine have not been modelled. The system can only 
monitor engine performance by on-site measurements of the operating parameters, but can not independently sinulate the performance of the engine.

Saravanamuttoo and Maclsaac (198j) introduced an engine thermodynamic modelling method for pipeline gas turbine diagnostics. To overcom: the difficulty of lack of component characteristic maps, especially the compressor maps, the authors made use of relative scaling of several compressor characteristic maps. They found that since each compressor is designed to meet the performance specification at engine design point, if the changes of relative flow and pressure ratio from surge to choke are plotted as a function of relative speed, these data collapse well enough to use an average curve for each. The significance of this paper is that it not only recommends using detailed engine component models for performance monitoring and engine diagnostics, but also gives the generalized component maps and shows the expected trends for particular types of engine deterioration.

A component-based engine nodel built by this method could be used to simulate the performance of an engine, and run over the entire operating range. It would be a computational representation of a real engine, and can easily simulate the performance at either design or off-design conditions, normal or deteriorated conditions It can be used for the purposes of validating EHM models, generating the baseline performance of a normal engine, creating fault matrices etc. It can provide an illdepth understanding of engine problems, which was not previously available to gas turbine operators. 


\section{Chapter 2}

\section{THE LM-1600}

The LM-1600, a product of General Electric's Aircraft Engine Business Group, is a simple cycle gas turbine with a twin-spool gas generator and a two-stage free power turbine. It is an industrial version of the F404, a highly reliable aircraft engine, with minimum change. As a high temperature and high pressure ratio gas turbine, the LM-1600 has cooled turbine nozzle and rotor blades, as well as variable compressor geometries. It is one of the highest efficiency gas turbines in the 12,000 to 18,000 shaft horse power range, and an excellent power plant for a variety of mechanical driving applications such as marine propulsion, electrical generation, compressor, pump and other loads.

\subsection{Components}

\section{Low Pressure Compressor}

The Low Pressure Compressor (LPC) is a three stage axial flow compressor. It is modified from the F404 by clipping its three fan stages by approximately four inches to reduce the flow path diameter. The pressure ratio across the LPC is about 4:1. Twenty-four variable Inlet Guide Vanes (IGV) are incorporated in the front frame 
to direct the inlet air into the LPC at the optimum angle for the particular engine operating condition.

\section{High Pressure Compressor}

The High Pressure Compressor (HPC) is identical to that of the F404. It has seven axial flow stages and gives a pressure ratio $5.5: 1$. Each of the first three stages cf the HPC is followed by a Variable Stator Vane stage (VSV). Sump pressurizing air and turbine cooling air are drawn from the inlet, middle and exit of the HPC.

\section{Combustor}

The annular combustor is identical to that of the F404. Fuel is distributed through eighteen fuel nozzles. The combustor case provides the airflow distribution around the combustor and the cooling air source for the High Pressure Turbine.

\section{High Pressure Turbine}

The High Pressure Turbine (HPT) is identical to that of the F404 except for minor changes to improve performance and for operation in the environmental conditions of industrial and marine applications. The HPT has a single axial stage with fully air cooled nozzle and rotor blades. The nozzle blades are cooled by a combination of convection, impingement and film cooling. The rotor blades are cooled by convection and film cooling.

\section{Low Pressure Turbine}

The Low Pressure Turbine (LPT) is modified from the F404 with the nozzle area reduced for better component matching. Like the HPT, the LPT also has only onc stage and air cooled nozzle and rotor blades. The cooling air passes through the LPT nozzle and rotor blades to perform convective cooling. 


\subsection{Cooling Air Flow Pattern}

The LPT nozzle and rotor blades are cooled by bleed air from the fourth stage of the HPC. The air enters through the top of each nozzle airfoil and exits at the root, then enters rotor blades through holes in the LPT blade dovetails, and exits at blade tip holes. This portion of the cooling air is a part of the working flow through the Power Turbine, but makes no work contribution to the gas generator turbines.

The cooling air for the HPT nozzle and rotor is drawn from the exit of the HPC. The nozzle cooling air exits at the surface of nozzle blades, and continuously expands through the HPT, the LPT and the Power Turbine, contributing work tc, all the three turbines. The rotor blade cooling air exits at the surface and tip holes of the HPT, contributing work output to the LPT and the Power Turbine, but not the HPT.

The sump pressurizing air, drawn from the inlet of $\mathrm{HPC}$, takes only a maximum of one percent of the inlet mass flow. Since it is a small quantity, non-continuous flow, it has been neglected in the model.

\subsection{Variable Bleed Valve}

Besides the IGV and VSV, the LPC discharge Yariable Bleed Valve (VBV) is another variable compressor geometry. The blow off manifolding is mounted in between the LPC and HPC. When the VBV opens, air blows off into the atmosphere to protect the compressor from surge. According to NOVA's operating data, the VRV is fully opened at engine starting, starts to close near half of the design load and is fully closed near the design point.

'The layout of the LM-1600 is shown in Fig. 1. The simplified flow pattern is sketched in Fig. 2. Station ${ }_{23}$ is the plane right after the $4^{\text {th }}$ stage of the HPC, where the cooling air for the LPT, $m_{1}$, is drawn. Station 45 is the gap between the stator and the rotor of the HPT, where the cooling air for the stator, $\boldsymbol{m}_{21}$, is completely mixed with the main stream working flow. 


\section{Chapter 3}

\section{DESIGN POINT CALCULATION}

The Design Point Calculation (DPC) is the first step in building the computational engine model. The main purpose of the DPC is to re-establish the design point of the engine from available information. Since the results will be incorporated int," the off-design calculation, the accuracy of the DPC will affect the accuracy of the engine model. So it is very important to ensure the DPC is done properly.

To re-establish the design point of the LM-1600, a DPC mathematical model has been set up first to work out the unknown design point parameters. Since there are more unknowns than valid equations, two parameters have to be empirically assumed to solve the model. The first assumed parameter is the compressor polytropic efficiency $\left(\eta_{c_{\infty}}\right)$. The second one is the High Pressure lurbine exit temperature $\left(T_{5}\right)$. Through a validating analysis, it is found that since the overall engine performance and some design point parameters are fixed, the adjustment of $\eta_{c \infty}$ will affect the total cooling air flow rate, and the adjustment of $T_{5}$ will affect the cooling air distribution. The validities of polytropic efficiency and the cooling air flow rates locate the DPC solution in a very narrow two-dimensional region. This means the solved design point is very close to the real one. 


\subsection{Basic Assumptions}

The following assumptions are used not only in the Design Point Calculation, but also throughout all the discussions and model programming in this thesis:

1). The flow in the gas turbine is one-dimensional.

2). The flow is steady.

3). The thermodynamic processes may be considered as adiabatic.

4). The flow behaves as a perfect gas with constant specific heat $\left(C_{p}\right)$ and specific heat ratio $(\gamma)$. The variation of these properties with temperature is considered as a less important factor and can be neglected.

5 ). The pressure loss in the combustion chamber is assumed to be constant as $5 \%$ of the combustor inlet pressure, i.e. $P_{4} / P_{3}=0.95$.

6). The mechanical transmission efficiencies of the LP-spool and the HP-spool are 0.99 .

\subsection{General}

The procedure of gas turbine design has been described in Chapter 1 of Gas Turbine Theory (Cohen, Rogers and Saravanamuttoo, 1987). The DPC discussed here is mainly covered within the Thermodynamic Design Point Studies.

\subsubsection{Concepts of Design Point Calculatıon}

When a new engine is being designed, the object of the (thermodynamic) Design Point Calculation (DPC) is to find the suitable design parameters to give the required power output with specified engine working conditions u..jer certain design limits.

For aircraft engines, the power output may be rated as thrust and/or shaft power at the cruise or sea level take-off condition. For marine and industrial engines, the power is ofter given as shaft power at sea level or International Standards Organization (ISO) standard condition. For both cases, factors such as maximum allowable 
turbine inlet temperature, and attainable rotational speed provide the design limits. With turbine inlet temperature specified, the designer can select a suitable pressure ratio to obtain the required level of $\mathrm{sfc}$, and this in turn will determine the mass flow. From these parameters, the component design, which includes aerodynamic analysis, stress analysis, geometry layout and material selection of the component elements, and further off-design analysis can be carried out. Design point optimization would be based on the considerations of engine life, weight, cost, overall efficiency and off-design behaviour. This type of DPC is referred to as the Real Engine DPC.

To build a computational model for an existing gas turbine, another type of DPC; has to be done, it may be referred to as the Model Engine DPC. In this case, the overall engine performance is usually known and some of the design parameters, but not all of them, can be found from literature published by the manufacturer. The object of this DPC is to determine those unknown parameters and mathematically define the engine. Since the solved parameters will be fed into the computational model for off-design calculation, it is very important to ensure it is done properly.

A design point calculation is based on thermodynamic relations of the working flow, and compatibility relations of work and flow between the components. If we set these relations as a DPC mathematical model, then the model would contain all the thermodynamic parameters, i.e. pressures, temperatures and mass flow rates at the inlet and outlet of each component.

In a Real Engine DPC, the designer can specify any of the unknowns needed for solving the model. In a Model Engine DPC, however, everything about the engine is set, and no modifications are necessary. But the analyst may face the difficulty that there are not enough valid parameters on hand to solve the model. In this case, some unknown parameters need to be estimated, trial and error or assumptions based un analysis and experience may be required.

\subsubsection{Known Design Point Parameters}

The known design point parameters of the LM-1600 come mainly from the manufacturer's sales brochure (GE, 1985). The engine is initially introduced with an ISO industrial base rating of 19,000 isentropic gas horsepower (IGHP), which corresponds to a gas turbine rating of 16,500 shaft horsepower. When sufficient operating 
experience is obtained, it is planned to uprate the LM-1600 to 20,700 IGHP $(18,000$ shaft horsepower). The flow pattern and station numbering of the engine are shown in Fig. 2. The DPC here is based on the introductory rating:

LPC Pressure Ratio 4.0 ;

IIPC Pressure Ratio 5.5 ;

HPT Inlet Temperture (TIT or $T_{4}$ ) $1,483 \mathrm{~K}$;

Exit Gas Temperature (EGT) of Gas Generator $\left(T_{6}\right) 1,014 \mathrm{~K}$;

Inlet Air Mass Flow $95 \mathrm{lb} / \mathrm{s}$;

Work Output 19,000 IGHP ;

SFC $0.343 \mathrm{lb} / \mathrm{IGHP}-\mathrm{hr}$;

Fuel Flow $\left(M_{f}\right) 6516 \mathrm{lb} / \mathrm{hr}$;

LP spool speed $12,500 \mathrm{rpm}$;

HP spool speed $15,600 \mathrm{rpm}$.

The positions of the variable conipressor geometries, IGV, VSV and VBV, vary with operating conditions. At the design point, the IGV and VSV are set at the normal open positions. The VBV is closed, hence no air is blown off. The simplified flow pattern is reduced from Fig. 2 to Fig. 3. The basic flows coilsidered here are the main stream working flow and the cooling air.

\subsubsection{Purpose of DPC for the Model of the LM-1600}

It will be explained in the next chapter that in the model of the LM-1600, a Hot End Method is used in component matching. Since the process is started from the turbine (hot) end, the turbine characteristics are initially required. Although a generalized turbine characteristic curve has been used, more detailed data of turbine swallowing capacities are required for scaling the curve to fit each individual turbine. Because no information on the HPT, LPT and Power Turbine characteristics has been published, this has to be determined in the DPC. In other words, one purpose of the DPC is to find the swallowing capacity of the engine by the know'n overall engine performance and operating parameters at the design point. As the results are fed into the engine model, off-design calculation can then be done.

The LM-1600 has cooled turbine blades. Though the cooling air flow pattern has 
been described by the manufacturer, accurate cooling air flow rates have not been found from any available information. Neglecting the cooling flows could cause large modelling error; so another goal of the DPC is to determine the cooling air flow rates at the design point, and set references for off-design cooling air flow simulation.

\subsection{DPC Model}

\section{Flm: Compatibility}

Keferring to Fig. 3, by the conservation of mass, we can get the following flow: compatibility relations at the design point:

$$
\begin{gathered}
M_{1}=M_{2} \\
M_{2}-m_{1}=M_{23} \\
M_{23}-m_{21}-m_{22}=M 3, \text { or } M_{1}-m_{1}-m_{21}-m_{22}=M_{3} \\
M_{3}+M_{f}=M_{4} \\
M_{4}+m_{21}+m_{22}=M_{5}, \text { or } M_{23}+M_{f}=M_{5} \\
M_{5}+m_{1}=M_{6} \text { or } M_{1}+M_{f}=M_{6} \\
M_{6}=M_{7}
\end{gathered}
$$

In this flow pattern, the HPT nozzle cooling air, $m_{21}$, js part of the working flow through all three turbines; the HPT rotor cooling air, $m_{22}$, is part of the working flow through LPT and Power Turbine; LPT cooling air, $m_{1}$, is part of working flow for the Power Turbine only.

\section{Work Compatibility}

The work compatibility equations at the design point would take the following forms:

LP Spool:

$$
W_{i p c}=W_{i p t}
$$


HP Spool:

$$
W_{h p c}=W_{h p t}
$$

Where:

$$
\begin{gathered}
W_{l p c}=M_{1} C_{p_{a}}\left(T_{2}-T_{1}\right) \\
W_{h p c}=M_{2} C_{p_{a}}\left(T_{23}-T_{2}\right)+\left(M_{2}-m_{1}\right) C_{p_{a}}\left(T_{3}-T_{23}\right) \\
W_{h p t}=\eta_{m}\left[m_{21} C_{p_{a}} T_{s}+M_{4} C_{p_{g}} T_{4}-\left(M_{4}+m_{21}\right) C_{p_{g}} T_{5}\right] \\
W_{l p t}=\eta_{m} M_{5} C_{p_{g}}\left(T_{5}-T_{6}\right)
\end{gathered}
$$

Considerations about the flow path and the temperatures used in these work equations are:

- The combustor exit gas enters the HPT with high temperature TIT, which is the firing temperature and has been given as $1483 \mathrm{~K}$.

- The HPT nozzle cooling air, $m_{21}$, exiting from the surface of the nozzle airfoil will be mixed with the main stream high temperature gas and will cool the temperature down to $T_{45}$. This mixed flow can be assumed to have the same specific heat as the combustion gas because $m_{21}$ is only a very small amount of flow.

- The mixed gas expands through the HPT rotor and is again mixed with the HPT rotor cooling air, $m_{22}$. The combined effect of expansion and mixing brings the temperature down to $T_{5}$ at exit of the HPT. The mixed gas will still have the property of the combustion gas.

- The HPT exit gas expands through the LPT and is once more mixed with the LPT cooling air, $m_{1}$, in the process. The temperature drops to $T_{6}$, which has been given as $1014 \mathrm{~K}$.

- All the gases expand through the Power Turbine. The temperature drops from $T_{6}$ to $T_{7}$

It is clear that different assumptions would lead to different forms of the work compatibility equations. But more accurate dividing of the mass flows and the temperatures does not necessarily give better results, even though the equations appear more complex.

\section{Combustion Energy Balance}

The combustion energy balance equation used in the DPC model is from Chappell and Cockshutt (1974) and written in the following form: 


$$
f=\frac{M_{f}}{M_{3}}=\frac{h_{4}-h_{3}}{E C I_{4}}=\frac{f\left(T_{4}\right)-f\left(T_{3}\right)}{E C l_{4}}
$$

where $h_{4}=f\left(T_{4}\right)$ and $h_{3}=f\left(T_{3}\right)$ are polynomial relationships between temperature and enthalpy. It may be worth pointing out that ihe accuracy of DPC is very sensitive $t$, the accuracy of combustion energy balance equation.

\section{Polytropic Compression}

Since the inlet air flow and fuel flow rates at the design point have been given, Equation (3.7) can be used to solve the mass flow rate at the exit of the HPC, $\mathrm{Mt}_{3}$, hence the total cooling air flow rate, which is the difference between inlet air flow, $M_{1}$, and $M_{3}$. But before applying Equation (3.7), the temperature at the exit of the $\mathrm{HPC}, T_{3}$, has to be found.

Assuming polytropic efficiency of the LPC and the HPC are same, $T_{3}$ can be found by polytropic compression equation:

$$
T_{2}=T_{1}\left(\frac{P_{2}}{P_{1}}\right)^{\frac{(n-1)}{n}}
$$

and

$$
T_{3}=T_{2}\left(\frac{P_{3}}{P_{2}}\right)^{\frac{(n-1)}{n}}
$$

where $\frac{(n-1)}{n}=\frac{1}{\eta_{c x}}\left(\frac{\gamma-1}{\gamma}\right)$.

To find $T_{23}$ in Equation (3.4), which is the temperature after the fourth stage of the HPC where the cooling air for the LPT is drawn, it is assumed that the total temperature of compressed air is uniformly raised through each of the seven LPC' compressor stages. So, $T_{23}$ can be estimated by:

$$
T_{23}=T_{2}+\frac{4}{7}\left(T_{3}-T_{2}\right)
$$




\subsection{Solution o: the DPC Model}

\subsubsection{Mr del Analysis}

The equaisons introduced above are the DPC model equations. The known parameters in this model are the design point parameters and overall engine performance given in Section 3.2. Since the consideration of flow compatibility has been built into the relation of work compatibility, we can think the valid model equations are Equation (3.1), (3.2) (which combine Equation (3.3) to (3.6)) and (3.7). According to the basic assumptions, the unknown terms contained in these model equations are mass flow rates and temperatures.

To solve the mass flows, the cooling air flow rates have to be found first. Since the inlet mass flow rate and the fuel flow rate at the design point are known, all the unknown mass flow rates in Equation (3.1) and (3.2) can be solved with the knowledge of $m_{1}, m_{21}$ and $m_{22}$, as well as flow compatibility relations.

As mentioned previously, the swallowing capacity of the engine has also to be determined at the DPC. One way to express the swallowing capacity is by the effective flow areas of the turbines. If we use a modified nozzle equation to simulate the generalized turbine characteristic, then the effective flow area could be found by total pressure ratio (or total temperature ratio) and mass flow rate across the turbine. If it is assumed that the HPT and the LPT have the same polytropic efficiency, by the help of the solved mass flow rates, the effective flow areas of the HPT and LPT can actually be solved by the HPT exhaust temperature $T_{6}$, and known temperature $T_{4}$ and $T_{6}$.

The LPC and HPC exit temperature, $T_{2}$ and $T_{3}$, can be determined by Equation (3.8) provided that the polytropic efficiency of the compressor is specified, and the $T_{23}$ can be found from Equation (3.9).

So in this DPC model, there are 5 independent unknowns, which are $m_{1}, m_{21}$, $m_{22}$ (or $\left.M_{3}\right), T_{5}$ and $\eta_{c \infty}$, within 3 equations. Theoretically if we can specify any two of the unknowns, the DPC model can be solved. 


\subsubsection{Solution Procedures}

By assuming $\eta_{c \infty}$ and $T_{5}$, the solution is carried out as follows:

(a). Assuming the same polytropic efficiency $\left(\eta_{c \infty}\right)$ for both the LPC and the $\mathrm{HPC}$, use Equation (3.8) to find $T_{2}$ and $T_{3}$;

(b). Find $M_{3}$ by Equation (3.7);

(c). Find $T_{23}$ by Equation (3.9);

(d). Substitute Equation (3.3) to (3.6) into (3.1) and (3.2), and assume $T_{5}$ to solve $m_{1}$ and $m_{21}$;

(e). Validating analysis: If the results obtained are not reasonable, modify the assumptions of $T_{5}$ and the polytropic efficiency, and repeat the calculation from step (a) to (d). The details of this analysis are given in the following section.

(f). Find $P_{4}$ by the known overal' pressure ratio and the assumption of constant pressure loss ratio across the combustor.

(g). Since the work output is given by isentropic gas horsepower, find $P_{6}$ by the isentropic expansion relation:

$$
P_{6}=P_{7}\left(\frac{T_{6}}{T_{7}}\right)^{\frac{\gamma}{(\gamma-1)}}=P_{1}\left(\frac{T_{6}}{T_{7}}\right)^{\left(\frac{\gamma}{\gamma-1)}\right.}
$$

where $T_{7}$ can be found by:

$$
\Psi_{\text {out }}=M_{7} C_{p_{g}}\left(T_{6}-T_{7}\right) \quad I G H P
$$

(h). Find polytropic efficiency of the HPT and the LPT by:

$$
\frac{(n-1)}{n}=\frac{\ln \left(T_{4} / T_{6}\right)}{\ln \left(P_{4} / P_{6}\right)}
$$

and

$$
\eta_{t \infty}=\frac{(n-1) / n}{(\gamma-1) / \gamma}
$$

(i). Find the effective flow areas of the turbines by turbine pressure ratios and the modified nozzle equation:

$$
P R_{h p t}=\frac{P_{4}}{P_{5}}=\left(\frac{T_{4}}{T_{5}}\right)^{\frac{n}{(n-1)}}
$$




$$
P R_{l p t}=\frac{P_{5}}{P_{6}}=\left(\frac{T_{5}}{T_{6}}\right)^{\frac{n}{(n-1)}}
$$

For unchoked turbine:

$$
\frac{M \sqrt{R T_{i n}}}{P_{i n} A}=\frac{1}{P R} \frac{\sqrt{\tau_{\gamma} \frac{2 \gamma}{-1)}\left(1-(1 / P R)^{(\gamma-1) / \tau}\right)}}{(1 / P R)^{(\gamma-1) / \gamma}}
$$

For choked turbine:

$$
\frac{M \sqrt{R T_{\text {in }}}}{P_{\mathrm{in}} A}=\left(1-\frac{\gamma-1}{\gamma+1}\right)^{\frac{\gamma}{(\gamma-1)}} \sqrt{\gamma \frac{(\gamma+1)}{2}}
$$

where $P R$ is the pressure ratio across the turbine, and $A$ is the effective flow area.

In this solution process, all the independent unknowns are found from Step (a) to (e). After Step (e), the geometry of the engine is actually fixed. To calculate the effective flow areas by the equations in the following steps is straightforward. It should be mentioned that since the nozzle characteristic is not an exact simulation of turbine, the calculated flow area may not be exactly the same as the real turbine especially when the turbine is unchoked; during most of the operation, however, the turbines are choked.

\subsubsection{Validating Analysis}

(Analysis explained in this section is carried on the solution step (e).)

Results obtained in Step (e) are based on assumed values of $T_{5}$ and $\eta_{c \infty}$. Since some operating parameters and overall engine performance at the design point are prescribed, the results will certainly match the specification. But not all of them are meaningful.

It is known that if a mathematical model contains two freely specified variables, the solution point is any point within a twc-dimensional domain; all the other calculated parameters will have different values corresponding to each different solution point. But for real gas turbine operation, every parameter has a valid value range; out of the range, the parameter would be meaningless. To establish the validity of the assumed parameters, the model initially locates the solution point within a linited 2-D domain. 


\begin{tabular}{||l||c|c|c|c|c||}
\hline Compressor Polytropic Efficiency & 0.86 & 0.88 & 0.90 & 0.92 & 0.94 \\
\hline \hline Total Cooling Air Flow (Ib/8) & 4.74 & 7.01 & 9.02 & 10.82 & 1243 \\
\hline Cooling Air/Inlet Air (\%) & 4.98 & 7.38 & 9.49 & 11.38 & 13.08 \\
\hline
\end{tabular}

\section{Table 1. Effect of Compressor Polytropic Efficiency on Cooling Air Flow}

\section{Restriction on $\eta_{\text {coo }}$}

Experience show's that the polytropic efficiency of a compressor varies around 0.88 to 0.92 . In this region, if the polytropic efficiency is high, the work required to drive the compressors would be low. Since the compressor pressure ratio and the engine work output are specified at the design point, this work reducion can only be realized by decrease of main stream working fow in the DPC model. Again, since the inlet air flow rate is also fixed at the design point, the reduction of the working flow would only mean that more inlet air is bled as the cooling air to match the given design condition. So, if a high polytropic efficiency is assumed, the total cooling air flow rate would also be high at the design point. This is shown in Fig. 4 and Table 1. For most high temperature gas turbines in service, the ratio of total cooling air flow to inlet air flow is around $10 \%$, which corresponds to $\eta_{\text {cos }}$ between 0.9 and 0.92 .

As the compressor polytropic efficiency is fixed, the total cooling air flow rate is then fixed in this DPC. Assumption of the HPT exit temperature, $T_{5}$, will not affect the total cooling air flow rate, but will affect its distribution.

Restriction on $T_{5}$

The initial $T_{5}$ searching domain is $\left(T_{6}, T_{4}\right)$. For steady state operation, cocling air flows for a high temperature gas turbine must be positive. For the LM-1600, the trends of cooling air flow rates versus $T_{5}$ are plotted on Fig. 5 to 7 with fixed values of $\eta_{c \infty}$ on each. It can be seen that only a very limited region is valid.

Firstly these figures show the effect of $T_{5}$ on cooling air distribution. With $T_{4}$ 
and $T_{6}$ fixed on both sides and the total cooling air flow rate fixed by $\eta_{c \infty}$, increase of $T_{b}$ woula lead to more cooling air being used for the LPT cooling $\left(m_{1}\right)$ ind less for HPT cooling ( $m_{21}$ and $m_{22}$ ). Decrease of $T_{5}$ would have the opposite effect. This trend is valid for all the values of $\eta_{c \infty}$, and the physical meaning is clear.

Secondly if $T_{5}$ is too low, the LPT cooling air appears to have negative flow rate; and if $T_{5}$ is too high, the HPT cooling air appears to have negative flow rates. This is clearly impossible. Since all three cooling air flows must be positive, the location of the valid $T_{8}$ domain should be in the corresponding region which is actually very narrow on each graph. This shrinks tle initial $T_{5}$ searching domain, $\left(T_{6}, T_{4}\right)$, dramatically.

The enlarged pictures of eligible $T_{5}$ regions are shown on Fig.8, 9 and 10. The valid $T_{5}$ region in each graph is about 6 to $8 \mathrm{~K}$ in width. When compressor polytropic efficiency is high, the valid $T_{5}$ region tends to be wider but the average temperature goes down.

Considering the HPT nozzle cooling air $m_{21}$, it is found that for each different compressor polytropic efficiency, the values of $m_{21}$ are different. The higher the polytropic efficiency, the higher the flow rate of $m_{21}$ will be. But within the valid $T_{5}$ region, $m_{21}$ does not change very much. The change of $T_{5}$ mainly affects the flow rates of $m_{1}$ and $m_{22}$. As $T_{5}$ increases, the variations of $m_{1}$ increasing and $m_{22}$ decreasing are nearly linear.

\begin{tabular}{||l||c|c|c|c||}
\hline \hline Cooling Air/Inlet Air & SPEY & TAY & RB211 & Average Ratio \\
\hline \hline HPT Nozzle (\%) & 6.5 & 6.0 & 8.0 & 2 \\
\hline HPT Rotor (\%) & 3.5 & 2.5 & 4.5 & 1 \\
\hline
\end{tabular}

Table 2. Analysis of Cooling Air Distribution

\section{Empirical Assumption}

In a gas turbine engine, most cooling air should be used to cool the high tem- 
perature parts, which are the HPT nozzle airfoils. The cooling air distributions in HPT for three types of gas turbines have been listed on Table 2 which comes from a personal communication to Prof. H.I.H. Saravanamuttoo at an ASME meeting. The average flow ratio of HPT nozzle cooling air to HPT rotor cooling air is about $2: 1$. If we use this ratio and make a further assumption that the cooling air flow for the HPT rotor and the LPT are nearly equal because of the lower temperature level for the LPT, then it will give us a ratio:

$$
m_{1}: m_{21}: m_{22}=1: 2: 1 \text {. }
$$

So the solution for $T_{5}$ will be the temperature corresponding to the cross point of $m_{1}$ line and $m_{22}$ line in Fig. 8, 9 and 10.

\begin{tabular}{||l|c|c|c||}
\hline \multicolumn{1}{|c|}{ Polytropic Efficiency } & 0.88 & 0.90 & 0.92 \\
\hline$T_{5}(\mathbf{K})$ & 1158.6 & 1155.1 & 1151.6 \\
\hline \hline Total Cooling Air (lb/s) & 7.01 & 9.02 & 10.82 \\
\hline$m_{1}(\mathbf{l b} / \mathbf{s})$ & 1.99 & 2.28 & 2.50 \\
\hline$m_{21}(\mathbf{l b} / \mathbf{s})$ & 2.99 & 4.49 & 5.85 \\
\hline$m_{22}(\mathbf{l b} / \mathbf{s})$ & 2.02 & 2.26 & 2.47 \\
\hline$m_{1}: m_{21}: m_{22}$ & $1: 1.5: 1$ & $1: 2: 1$ & $1: 2.3 \cdot 1$ \\
\hline
\end{tabular}

Table 3. Analysis of DPC Results

For different compressor polytropic efficiencies, the results of cooling air flow rates are listed in Table 3. It shows that the one with polytropic efficiency equals (0.90) and $T_{5}$ equals $1155^{\circ} \mathrm{K}$ gives the best agreement with the assumed flow distribution. 


\subsection{Conclusions}

The Design Point Calculation of the LM-1600 is based on published information about this engine. The main reference is the manufacture-'s sales brochure (GE, 1987).

Basic equatirms used in the DPC are thermodynamic relations of the working flow, compatibility elations of flow and work between the components, and energy balance equation of combustion. Because there are two more unknowns, this equation set only locates the design point in a two-dimensional domain which is restricted by the validities of the two parameters.

Due to the requirement for positive cooling air flow, this restricted 2-D domain shrinks and becomes very small. Variation of design point in this domain would not change the specified engine performance and design point parameters, but would affect the total cooling air flov: rate, the cooling flow distribution, and slightly the turbine flow areas. On the one hand, this might affect the predictions of the offdesign calculation. On the other hand, any meaningful selection of the design point in this domai:- hnuld not cause large difference because this domain is very small. The final solution here is derived from analysis and an empirical assumption. Although the computer model of the LM-1600 based on this DPC has shown fairly good agreemen: with the real engine, as shown in Chapter 5 , the program still leaves room of improvement of the $\mathrm{DPC}$ if more information became available. 


\section{Chapter 4}

\section{HOT END METHOD}

For a twin-spool land based gas turbine, the steady state engine model contains 10 equations which are governing equations of the component matching process and the component model equations of the turbines. There are 11 independent variables in this mathematical model, which represent the properties of the working flow at inlet and outlet of each of the engine components. Theoretically if one of the variables is given or known, the mathematical model can be uniquely solved. Physically this means that corresponding to each steady state operating point of a gas turbine, there is a unique set of gas property data, or operating parameters. Since these ten equations are highly nonlinear, it is very hard to solve them directly. Numerical solution starting at the Hot End has been successfully used, speeding up the component matching computation.

\subsection{Requirements for Engine Modelling}

As the engine design condition is mathematically set up, the next step is to find the mathematical engine model for off-design performance simulation. The requirements for this model would be:

1. The model should be able to describe the engine performance over the full operating range. 
2. The modelling equations should contain clear physical meaning.

3. The computational model should be similar to the real engine both in its performance and operation. It should be able to give the user enough engine running information and operating flexibility to analyze the real engine and couple the model with the existing field environment.

In general, the following work is involved in building an engine model:

1. To find the mathematical model. In doing this, the use of over complicated mathematical equations should be avoided, which will not only be hard for the solution process, but also make the physical meaning of the model unclear.

2. To find a solution method. The model should at least be solvable. The complexity of the solution would restrict the model application.

3. To program the model in the way of meeting user's requirements.

\subsection{Mathematical Model and Component Matching Process}

\subsubsection{Governing Equations and Component Matching Calculation}

A steady state gas turbine model is mainly based on the concept of component matching process. This process is governed by the equations of flow compatibilities and work compatibilities between the engine components. For a land based twinspool gas turbine, as sketched in Fig. 11, the governing equations are listed below. If the working condition of an engine satisfies all these equations, the engine operates in a steady state condition. Otherwise, it is in a trarient condition.

Flow compatibility between LPC and HPC:

$$
\frac{M_{a} \sqrt{T_{2}}}{P_{2}}=\frac{M_{a} \sqrt{T_{1}}}{P_{1}} \frac{P_{1}}{P_{2}} \sqrt{\frac{T_{2}}{T_{1}}}
$$


Flow compatibility between $\mathrm{HPC}$ and HPT:

$$
\frac{M_{g} \sqrt{T_{4}}}{P_{4}}=\frac{M_{g}}{M_{a}} \frac{M_{a} \sqrt{T_{2}}}{P_{2}} \frac{P_{2}}{P_{3}} \frac{P_{3}}{P_{4}} \sqrt{\frac{T_{4}}{T_{2}}}
$$

Flow compatibility between HPT and LPT:

$$
\frac{M_{g} \sqrt{T_{5}}}{P_{5}}=\frac{M I_{g} \sqrt{T_{4}}}{P_{4}} \frac{P_{4}}{P_{5}} \sqrt{\frac{T_{5}}{T_{4}}}
$$

Flow compatibility between LPT and Power Turbine:

$$
\frac{M_{g} \sqrt{T_{6}}}{P_{6}}=\frac{M_{g} \sqrt{T_{5}}}{P_{5}} \frac{P_{5}}{P_{6}} \sqrt{\frac{T_{6}}{T_{5}}}
$$

Work compatibility between LPC and LPT:

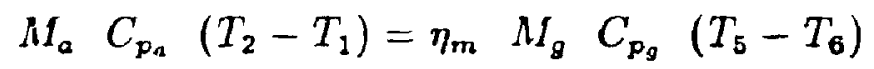

Work compatibility between HPC and HPT:

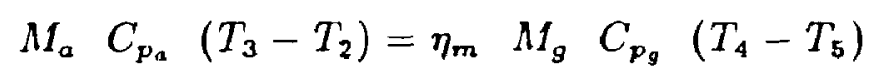

Component characteristics of a gas turbine can usually be expressed as Fig. 12. If the characteristic maps of a gas turbine are available, the component matching calculation can be done by using a trial and erroi procedure with the help of the governing equations and some graphic work. This method has been introduced by Cohen, Rogers and Saravanamuttoo (1987). To build an engine computational model by this method, the following work should be done:

1. To start the calculation by establishing the HPC running line, the assumption of LPT choking is necessary. Several one-dimensional iterations along each HPC: speed line are required. Cross point calculation and curve fit, which are computerized graphic work, are essential.

2. The compressor characteristic maps have to be incorporated into the computer program. To ensure the accuracy of the graphic calculation, the curves should be stored either in a detailed data file or by different polynomials.

3. The component matching starts by guessing the LPC operating point. With no knowledge of the LPC running line, this calculation will be a two-dimensional 
iteration if no operating parameter of the LPC, i.e. the pressure ratio, the nondimensional mass flow or the rotational speed, is specified. If it is wanted to create the LPC runing line to restrict the searching domain, several long loop iterations along each LPC speed line have to be done.

The trial and error method is an efficient method in component matching calculation, but it is originally designed for manual work with available component characteristic maps. It may be found that a gas turbine modelling program based on this method tends to be long, the requirement of memory space is large and computing time is long too. If the gas turbine is in multi-spool configuration and has complex flow pattern or variable geometry, the difficulty will be significantly increased. If the modelling of deteriorated engine conditions and variable inlet conditions are required, which will vary the compressor running lines, to carry out the work on a single PC floppy disc may even not be possible.

Another problem being faced here is that the component characteristics are usually unavailable because engine manufacturers often regard the maps as proprietary, especially the compressor characteristics. This difficulty led to some other methods being developed. Among them, a significant contribution made by Wittenberg (1976) is an example.

In Wittenberg's method, a generalized turbine curve and estimated component efficiencies are used instead of real component maps. The calculation is restricted to thermodynamic relationships. Like the trial and error method, choking of the LPT is also assumed for a twin-spool gas turbine. This assumption fixes the operating condition of the HPT, so the component matching is actually simplified to a solving of the LP-spool work compatibility relation, which is graphically expressed as a cross point of two curves for the LPC and LPT. The LPC curve varies with inlet condition. The cross point has to be found by a numerical procedure.

Wittenberg's method brings more mathematics into the calculation. This certainly makes the component matching calculation much easier. But as the solution method is still based on graphic process, it does not have the required flexibility for engine modelling, and is still not convenient for computation. A question arising here is could a further modified method be made to improve the model availability and make it more suitable for computing. 


\subsubsection{Governing Equation Analysis}

When an engine is run at a steady state condition, there is a unique set of operating parameters corresponding to it. These parameters are thermodynamically coupled together. An engine operator can control the engine by setting a value for one of the parameters, such as fuel flow rate, and observe the responses of others. But he can not set two values for two parameters without any physical change of the cugine, if the values disobey the existing thermodynamical and compatibility relations. For example, the operator can not change the LP-spool speed and HP-spool speed in different directions without change of turbine geometry; he can not decrease the fuel flow and increase the exhaust gas temperature with the power turbine flow area fixed. This physical engine feature can be mathematically expressed as a set of $N$ equations with $N+1$ unknowns. If one of the unknowns is specified, the equation set can be uniquely solved. The mathematical feature of this equation set represents the physical behavior of an engine. This equation set and the unknowns are the mathematical engine model we are seeking.

The existing equations are the governing equations of component matching. To analyze its structure, it can be found that:

- The pressure ratios, temperature ratios and non-dimensional mass flow rates (axial Mach Number) in the compressors are linked by Equation (4.1).

- The corresponding properties for the turbines are linked by Equation (4.3).

- The properties on both sides are interconnected by Equation (4.2), (4.5) and (4.6).

- If the operating condition of a gas generator is fixed, the operating condition of the power turbine then is fixed, or vice versa. This is partially expressed by Equation (4.4).

This structure simply tells us that if we can determine the gas properties in the compressors, we actually determine the properties in the turbines too. In other words, if we fix the working conditions of the turbines, we also fix the working conditions of the compressors. In the case of the power turbine, if we fix its working condition, we also fix the required working condition of the gas generator.

Recalling the thermodynamic expressions for compression (compressor) and expansion (turbine), the governing equations can be re-written as Equation (4.1.1) to (4.6.1). 


$$
\begin{gathered}
\frac{M_{a} \sqrt{T_{2}}}{P_{2}}=\frac{M_{a} \sqrt{T_{1}}}{P_{1}} \frac{P_{1}}{P_{2}} \sqrt{1+\frac{1}{\eta_{l p c}}\left[\left(\frac{P_{2}}{P_{1}}\right)^{\epsilon_{a}}-1\right]} \\
\frac{M_{g} \sqrt{T_{4}}}{P_{4}}=\frac{M_{g}}{M_{a}} \frac{M_{a} \sqrt{T_{2}}}{P_{2}} \frac{P_{2}}{P_{3}} \frac{P_{3}}{P_{4}} \sqrt{\frac{\left(T_{4} / T_{1}\right)}{1+\frac{1}{\eta_{l p c}}\left[\left(\frac{P_{2}}{P_{2}}\right)^{\epsilon_{a}}-1\right]}} \\
\frac{M_{g} \sqrt{T_{5}}}{P_{5}}=\frac{M_{g} \sqrt{T_{4}}}{P_{4}} \frac{P_{4}}{P_{5}} \sqrt{1-\eta_{h p t}\left[1-\left(\frac{1}{P_{4} / P_{5}}\right)^{\epsilon_{g}}\right]} \\
\frac{M_{g} \sqrt{T_{6}}}{P_{6}}=\frac{M_{g} \sqrt{T_{5}}}{P_{5}} \frac{P_{5}}{P_{6}} \sqrt{1-\eta_{l p t}\left[1-\left(\frac{1}{P_{5} / P_{6}}\right)^{\epsilon_{g}}\right]} \\
\frac{M_{a} C_{p_{a}}}{\eta_{m} M_{g} C_{p_{s}}} \frac{T_{1}}{\eta_{l p c}}\left[\left(\frac{P_{2}}{P_{1}}\right)^{\epsilon_{a}}-1\right]=\eta_{l p t} T_{4}\left(1-\eta_{h p t}\left[1-\left(\frac{1}{P_{4} / P_{5}}\right)^{\epsilon_{g}}\right]\right)\left[1-\left(\frac{1}{P_{5} / P_{6}}\right)^{\epsilon_{g}}\right] \\
\frac{M_{a} C_{p_{a}}}{\eta_{m} M_{g} C_{p_{s}}} \frac{T_{1}}{\eta_{h p c}}\left(1+\frac{1}{\eta_{l p c}}\left[\left(\frac{P_{2}}{P_{1}}\right)^{\epsilon_{a}}-1\right]\right)\left[\left(\frac{P_{3}}{P_{2}}\right)^{\epsilon_{a}}-1\right]=\eta_{h p t} T_{4}\left[1-\left(\frac{1}{P_{4} / P_{5}}\right)^{\epsilon_{s}}\right]
\end{gathered}
$$

Since the inlet conditions are known, if we assume that all the efficiencies can be found by some means, and fuel addition can be neglected, the relationship of these governing equations and unknowns can be sketched as Fig. 13. To analyze the figure, it can be found that:

- There are 10 independent variables in this component matching calculation, they are:

$$
\begin{gathered}
\frac{M_{a} \sqrt{T_{1}}}{P_{1}}, \frac{M_{a} \sqrt{T_{2}}}{P_{2}}, \frac{M_{g} \sqrt{T_{4}}}{P_{4}}, \frac{M_{g} \sqrt{T_{5}}}{P_{5}}, \frac{M_{g} \sqrt{T_{6}}}{P_{6}} \\
\frac{P_{2}}{P_{1}}, \frac{P_{3}}{P_{2}}, \frac{P_{4}}{P_{5}}, \frac{P_{5}}{P_{6}}, \quad \text { and } T_{4} .
\end{gathered}
$$


There are only 6 independent equations. To solve the model, we need 4 more equations, or 3 more equations and 1 specified unknown, or 2 more equations and 2 specified unknowns, or 1 more equation and 3 specified unknowns etc.

- From the above argument, if we specify more than one unknowns which are actually operating parameters of the engine, the specified unknowns should be thermodynamically related. In other words, the specified unknowns should correspond to the same possible engine working point. If not, the model would not give a valid solution.

- Even if we have enough equations, we may not necessarily be able to solve the model because these equations are highly non-linear and highly inter-connected. Solving a set of highly non-linear equations would be not easy.

- If two unknowns in one of the blocks of Fig. 13 are fixed by some means, physically it says the working condition of that component is known; the unknowns in adjacent blocks may still not be able to be solved because the problem of more unknowns and less equations still exists. For example, if the two unknowns in HPT block are fixed, to the left, there are three unknowns $\left(T_{4}, P_{3} / P_{2}\right.$ and $\left.M_{2} \sqrt{T_{2}} / P_{2}\right)$ in two equations $((4.2 .1)$ and (4.6.1)); to the rigit, Equation (4.3.1) can be used to solve $M_{5} \sqrt{T_{5}} / P_{5}$, but $P_{5} / P_{6}$ still can not be found if the relation between the two unknowns in the LPT is missing.

So the key things in developing this component matching model into a mathematical engine model seem to be: 1) how to find more equations; 2) where to start the solution process, and how to avoid solving the non-linear equation set.

\subsection{Hot End Solution Process}

\subsubsection{Generalized Turbine Model}

Previous research has shown that the characteristic of a turbine can be generalized as a single curve, $i . s$ the performance of a turbine is almost independent of its rotational speed. The significance of this turbine curve is not only its simplicity, but also it reduces the location domain of the turbine working point from a 2-D area to a 1-D curve. Although some shortcomings of this simple expression will be shown 
later, it is certainly convenient to gas turbine simulation.

This generalized turbine curve can be fitted by a polynomial or part of elliptical curve, or can be simulated by a modified nozzle equation. The polynomial and nozzle equation methods have some advantages and disadvantages as listed in Table 4. The elliptical curve fitting, i.e. so called Law of Ellipse, contains the shortcomings of both and has not been considered here. But no matter which method is used, as long as the pressure ratio across the turbine is known, the non-dimensional mass flow can be found, hence the working condition for that turbine is fixed. It should be realized that the reverse is not true, when the turbine is choking.

\begin{tabular}{|c|c|}
\hline Modified Nozzle Equation & Polyromial Curve Fit \\
\hline $\begin{array}{l}\text { Advantages: } \\
\text { 1. Easy to program. } \\
\text { 2. Turbine characteristics } \\
\text { are not needed. } \\
\text { 9. Corputing time is short. } \\
\text { 4. Easy to simulate variable } \\
\text { geometry turbine. }\end{array}$ & $\begin{array}{l}\text { Advantages: } \\
\text { 1. Turbine characteristics can } \\
\text { be in different shapes. } \\
\text { 2. Turbine flow area does not } \\
\text { needed to be specified. }\end{array}$ \\
\hline $\begin{array}{l}\text { Disadvantages: } \\
\text { 1. Flow area of turbine needs } \\
\text { to be specified. } \\
\text { 2. All turbine curves have } \\
\text { same shape. }\end{array}$ & $\begin{array}{l}\text { Disadvantages: } \\
\text { 1. Turbine characteristics are } \\
\text { needed. } \\
\text { 2. Program is relatively long, } \\
\text { and computing time. } \\
\text { 3. The equation does not } \\
\text { have any physical meaning. }\end{array}$ \\
\hline
\end{tabular}

Table 4. Comparison of Different Turbine Simulating Methods 
The generalized HPT and LPT turbine characteristics add two more equations to the cornponent matching calculation, which are Equation (4.7) and (4.8). Hence the equation and unknown relation becomes the one sketched in Fig. 14. From previous discussion, if we can specify two related unknowns, we can solve the model.

To assume the LPT is choking hence $M_{5} \sqrt{T_{5}} / P_{5}$ is fixed, using relation (4-3) and (4-7) to find $P_{4} / P_{5}$ and $M_{4} \sqrt{T_{4}} / P_{4}$, and using Equation (4.2), (4.6) with the help of some graphic work to establish HPC running line is the first step of the trial and error method. Then the engine working condition (or working point) can be found by the second specification through the component matching. This approach reduces the iteration on the HPC map. The assumption of LPT choking is found to be true for most gas turbines over the useful running range.

Wittenberg's simplified method (1976) assumes the same polytropic efficiency for LPC and HPC, and the same polytropic efficiency for HPT and LPT. This inplies the twin-spool gas generator actually becomes one-spool. Similarly it assumes choked Power Turbine at design and off-design conditions, which means the working point of the gas generator turbine rever changes. Then it uses the designed turbine pressure ratio as the second known to solve the model.

When two unknowns need to be specified but we can only specify one, the solution is not bound. The calculation can only be carried out by the help of some other methods or assumptions. But if we can find one more equation and specify only one unknown, the solution should always be valid.

\subsubsection{Power Turbine Pressure Ratio, $P_{6} / P_{7}$}

It is interesting that there is another gas property parameter that has not been involved in the mathematic model. This is $P_{6} / P_{7,}$, the pressure ratio across the Power Turbine. If we treat the diffuser as part of the Power Turbine, we can define that $P_{6}$ is the inlet stagnation pressure of the Power Turbine and $P_{70}$ is the static back pressure acting at the exit of the turbine.

An analysis shows that if we include the Power Turbine and the concept of pressure equilibrium into our engine model, two additional equations can be added. The first one is the generalized turbine characteristic of the Power Turbine, Equation (4.9). The second one is the pressure equilibrium equation, Equation (4.10). Since these two equation are both related to $P_{6} / P_{7}$, it not only compensates the appear- 
ance of $P_{8} / P_{7}$, in the model, but also adds one more equation. As shown in Equation (4.10), if we neglect the losses and kinetic energy at the inlet of the LPC, as well as the kinetic pressure at the exit of the power turbine, $P_{7}$, equals to $P_{1}$. If we need a more accurate calculation, these neglected terms can still be brought back into the pressure equilibrium consideration.

$$
\begin{gathered}
\frac{M_{0} \sqrt{T_{4}}}{P_{4}}=f_{n}\left(\frac{P_{4}}{P_{5}}\right) \\
\frac{M_{g} \sqrt{T_{5}}}{P_{5}}=f_{n}\left(\frac{P_{5}}{P_{6}}\right) \\
\frac{M_{g} \sqrt{T_{6}}}{P_{6}}=f_{n}\left(\frac{P_{6}}{P_{7}}\right) \\
\left(\frac{P_{3}}{P_{2}}\right)=\frac{\left(P_{4} / P_{5}\right)\left(P_{5} / P_{6}\right)\left(P_{6} / T_{10}\right)}{\left(P_{2} / P_{1}\right)\left(P_{4} / P_{3}\right)}
\end{gathered}
$$

Now we have 11 unknowns and 10 equations. These two additional equations, (4.9) and (4.10), and the pressure ratio $P_{6} / P_{76}$, as well as previously mentioned equations and unknowns give the full mathematic model of twin-spool gas turbine, which is shown in Fig. 15. Using this model, we need to specify only one unknown. No iteration is necessary and the solutions would always be valid. Or physically, the solution points always lie on the engine running line.

\subsubsection{Initiate the Solution Process}

Where to start the solution and which unknown should be chosen as the specified one are the same question; avoiding the non-linear equation set is the basic consideration.

Considering the characteristics of the mathematic model, it is found that if the specified unknown is in the compressors, we will face the non-linear equation set, since all the parameters in the compressors are highly connected. With no information on the compressor running line, the solution point lies in a two-dimensional domain.

If the specified unknown is in the turbines, the situation becomes quite different. Referring to Fig. 15, it can be found: 
- If we specify a non-dimensional mass flow in one of the turbine blocks, we may not be able to find the pressure ratio in the same block and the two unknowns in the right block, if the turbine is choked. But we can always find the two unknowns in the left turbine block by flow compatibility and the generalized turbine equation.

- If we specify a pressure ratio in a turbine block, we can always find the nondimensional mass flow in the block, the two unknowns in the left turbine block and the non-dimensional mass flow in the right block, no matter whether these turbines are choked or not. But we may not be able to find the pressure ratio in the right block if that turbine is choked.

This analysis tells us that specifying pressure ratio is always better than specifying non-dimensional mass flow, and specifying a parameter in the right block is better than doing one on the left. The analysis concludes that the pressure ratio of the Power Turbine is the best choice, and the solution process should be started from the engine Hot End by specifying $P_{6} / P_{7}$.

\subsubsection{Solution Procedure}

The procedure of the Hot End Solution Process is really simple. Referring to Fig. 15 , the procedure is:

a. Specify $P_{6} / P_{78}$, and use Equation (4.9) to find $M_{6} \sqrt{T_{6}} / P_{6}$;

b. Use Equation (4.4.1) and (4.8) to find $P_{5} / P_{6}$ and $M_{5} \sqrt{T_{5}} / P_{5}$;

c. Use Equation (4.3.1) and (4.7) to find $P_{4} / P_{5}$ and $M_{4} \sqrt{T_{4}}, P_{4}$; we then fix the working condition of the turbines.

d. Solve work compatibility equations (4.5.1) and (4.6.1). There are three un. knowns, $T_{4}, P_{2} / P_{1}$ and $P_{3} / P_{2}$, in these two equations. Substituting the pre-solved turbine pressure ratios into Equation (4.10), the $P_{3} / P_{2}$ in Equation (4.6.1) can be expressed as function of $P_{2} / P_{1}$ and the solved pressure ratios. Then there are only two unknowns, $T_{4}$ and $P_{2} / P_{1}$, in Equation (4.5.1) and (4.6.1).

Using the pre-solved pressure ratios, we can rewrite the LP-spool work compatibility equation (4.5.1) in the following simplified form:

$$
\left[\left(\frac{P_{2}}{P_{1}}\right)^{c_{a}}-1\right]=A T_{4}
$$


Substitute (d-1) to (4-6-1) we can get the simplified HP-spool work comf-tibility equation that is a second order polynomial and contains only one unknown:

$$
B T_{4}{ }^{2}+C T_{4}+D=0
$$

Where $A, B, C$ and $D$ are coefficients derived from the pre-solved values and knowns.

There are two roots of Equation (d.2). One of them is the solucion of $T_{1}$. The other one is negative. Although physically meaningless, the negative root is an "imaginary temperature" of a "reverse" gas turbine; i.e. "compressor" expands the inlet air, "combustor" takes energy out of flow and the temperature drops to the imaginary value, then "turbine" compresses the flow back to ambient pressure. lnstead of hot gas exhausting, it exhausts cold gas. The T-S diagram of this reverse engine is opposite down to the one of real gas turbine. Any way, to identify the solution of $T_{4}$ is really straightforward. Once we have set the solving procedure of the simplified work compatibility relations into the program, which is quite short, the solution process carried out by computer is very fast.

e. Finally solving $P_{3} / P_{2}$ by $(4.10), M_{2} \sqrt{T_{2}} / P_{2}$ by $(4.2 .1)$ and $M_{1} \sqrt{T_{1}} / P_{1}$ by (4.1.1) are straightforward.

The solution of the mathematic model will contain all the gas properties at each component inlet and outlet. No restrictions about engine running condition are necessary. If the running lines of the compressors are required, one can just give a series of values of $P_{6} / P_{7,}$, from minimum to naximum possible value, to the program. Then connecting the calculated working points of both compressors, the running lines of $\mathrm{LPC}$ and $\mathrm{HPC}$ can be obtained at the same time.

\subsection{One-Dimensional Seerching}

\subsubsection{Relationships between $P_{6} / P_{7}$ and Other Parameters}

At each engine steady state working point, there is only one set of gas property data corresponding to it. If we choose a value of power turbine pressure ratio, 


\begin{tabular}{|c|c|c|c|c|c|c|}
\hline$P_{6} / P_{7}$ & 1.2 & 1.4 & 1.6 & 1.8 & 2.0 & 2.4 \\
\hline$P_{2}$ (Psia) & 24.98 & 33.03 & 38.52 & 42.06 & 44.22 & 49.11 \\
\hline$T_{2}(K)$ & 355 & 395 & 418 & 432 & 440 & 457 \\
\hline$P_{3}$ (Psia) & 67.62 & 96.10 & 116.8 & 133.2 & 146.9 & 175.8 \\
\hline$T_{3}$ (Psia) & 522 & 596 & 641 & 673 & 697 & 745 \\
\hline$P_{4}$ (Psia) & 64.24 & 91.29 & 111.0 & 126.6 & 139.5 & 167.0 \\
\hline$T_{4}(K)$ & 843 & 983 & 1088 & 1177 & 1257 & 1404 \\
\hline$P_{5}$ (Psia) & 26.52 & 3644 & 44.30 & 50.59 & 55.76 & 66.76 \\
\hline$T_{5}(K)$ & 696 & 806 & 892 & 965 & 1030 & 1151 \\
\hline$P_{6}$ (Psia) & 17.64 & 20.58 & 2352 & 2646 & 29.40 & 3528 \\
\hline$T_{6}(K)$ & 637 & 711 & 777 & 838 & 897 & 1002 \\
\hline$P_{7,}\left(P_{s i a}\right)$ & 14.7 & 14.7 & 14.7 & 14.7 & 14.7 & 14.7 \\
\hline$T_{7}(K)$ & 612 & 661 & .01 & 737 & 771 & 828 \\
\hline$M_{1} \sqrt{T_{3}} / P_{1}$ & 181.6 & 239.0 & 276.1 & 302.7 & 322.9 & 365.9 \\
\hline$M_{2} \sqrt{T_{2}} / P_{2}$ & 118.7 & 124.6 & 127.0 & 129.6 & 132.7 & 138.0 \\
\hline$M_{4} \sqrt{T_{4}} / P_{4}$ & 71.1 & 71.1 & 71.1 & 71.1 & 711 & 71.1 \\
\hline$M_{5} \sqrt{T_{5}} / P_{5}$ & 156.5 & 161.3 & 161.2 & 161.0 & 161.1 & 161.1 \\
\hline$M_{6} \sqrt{T_{6}} / P_{6}$ & 225.0 & 268.3 & 283.3 & 286.8 & 285.0 & 284.4 \\
\hline$M(1 b / s)$ & 157 & 207 & 239 & 262 & 280 & 317 \\
\hline$P_{4} / P_{5}$ & 2.42 & 2.51 & 2.51 & 2.50 & 2.50 & 2.50 \\
\hline$P_{5} / P_{6}$ & 1.50 & 1.77 & 1.88 & 1.91 & 1.89 & 1.89 \\
\hline$T_{6}-T_{7}(K)$ & 25 & 51 & 76 & 101 & 126 & 173 \\
\hline & & & & & & \\
\hline
\end{tabular}

Table 5. Relationships between $\frac{P_{c}}{P_{70}}$ and Other Parameters 
$P_{6} / P_{7 d}$, we then fix the working point of the whole engine. Mathematically, the other parameters should be single value functions of the $P_{8} / P_{78}$. The shortcoming of this is that we can only determine tile value of the $P_{6} / P_{7 a}$, but we do not know what values other parameters will have. We have only one controllable parameter for this model engine.

In practice, it may be required to sct a control at the Cold End of a gas turbine, Euch as we may want to control mass flow rate or TIT, or we may have good measurements of OPR and fuel flow, we may know the work output and so on. For an engine monitored by field instrumentation, $P_{6} / P_{7}$, is not necessarily the best measured parameter. In order to couple the hot end model with the field environment, we should enlarge the applicability of the model.

T. ble 5 and Fig. 16 show some results given by an analysis. It shows clearly that most of the parameters tend to increase as $P_{6} / P_{7}$ increases, i.e. they keep moatotonic increasing relationships with $P_{6} / P_{78}$.

\subsubsection{One-Dimensional Searching Technique}

The existence of the monotonic increasing relationships allows a numerical method of One-Dimensional Searching to be used. In a 1-D searching computation, the program will adjust the variable by the sign of the error between calculated value and the specified value, search the solution point along a 1-D domain, and finally find the solution with the required accuracy. In our gas turbine model, the 1-D dumain is the single curve of the generalized Power Turbine characteristic.

If some other parameters instead of $P_{8} / P_{7}$, are required to have a fixed value, the 1-D searching program will carry out the requirement by the following steps.

A. Specify the parameter, for example mass flow rate Ms (specified value);

B. Give ray value to $P_{6} / P_{7}$ (variable);

C. The program will calculate a mass flow rate $M$ (calculated value) as a function of the $P_{6} / P_{70}$;

D. If (Ms-M) is greater than zero, it means that the value of $P_{6} / P_{7}$, should be adjusted upward. If (Ms-M) is less than zero, it means that the value of $P_{6} / P_{7}$, should be adjusted downward;

E. The program adjusts $P_{6} / P_{7 s}$ and repeats the calculation from step (C). 
The 1-D searching program will first locate the solution in a certain region, then reduce the width of the 1-D domain by each calculation run. rinally the working point corresponding to the specified Ms will be found with the required accuracy In this area, many simple and efficient optimization techniques can be used to speed up the searching speed. But this is beyond the topic of this thesis.

The availability of the One-D Searching Technique enlarges the applicability of the Hot End Solution Process. The combination of the hot end solution process and the 1-D searching is the Hot End Method. By this method, almost any thermal property of the gas turbine can be chosen as the specified unknown. If the relationship between a thermal parameter and a mechanical parameter, such as 50 . tor rotational speed, is known, the mechanical parameter also becomes contrclable. For users who have better mechanical measurement than thermal measuremen., this may be useful. One thing worth mentioning is that for each gas turbine, its swallowing capacity is fixed. If the specified value is beyond the gas turbine's capability, the program will not work, and the real engine would also not work at that condition.

(The controllable parameters in the model of LM-1600 are listed on Table 7 of Chapter 6.)

\subsection{Overview of Hot End Method}

The Hot End Method introduced here is a modification of Wittenberg's method. The modelling equations used in these two methods are basically the same. Although the two methods are initially created for the situation that component characteristic maps are absent, both methods can be applied with greater accuracy by correcting for the variation of efficiency if the maps are available.

The major difference between the Hot End Method and Wittenberg's method is that the Hot End Method solves the model by a straight mathematical routine initiated from the hot end and works backward; the two work compatibility equations are solved by a set of two equations with two unknowns. Wittenberg's method solves the model by finding the cross point between two curves, which is actually the solution of the work compatibility of the LP-spool. The assumption of LPT choking is essential for this method. 
The advantages of the Hot End Method are:

(1) The whole process contains only simple mathematical calculations. It does not require any unnecessary graphic work or matrix calculations which are commonly found in many currently used engine models, so is easier for application. The process is very suitable for computation because the program is relatively short and the mathemptical solution takes much less time than either graphic or matrix calculations. This is especially useful when a portable $\mathrm{PC}$ model is required by user.

(2) Because of its simplicity, the physical meaning of the process, which is based on the concept of steady state operation of gas turbine, is clear.

(3) Since all points along the power turbine characteristic line are possible operating points, the solved turbine working points mould always be valid. The solved compressor working points would be along the running line if the efficiency is accurately assumed, or very close to the line if a compressor map is available for further accuracy correction. The correction is made only to the efficiencies contained in the work compatibility equations, (d.1) and (d.2). It does not need to go back to the beginning of the calculation, so convergence occurs very rapidly.

(4) Though in most cases of interest, the LP turbines are choked, the assumption of LP turbine choking is not needed in the Hot End Method. This is still true even if the LP turbine is largely unchoked at very low output. The solution process is valid for the full range of engine operation.

(5) Since the method is not initiated from the compressor end, any variation of compressor running lines would not affect component matching. For a deteriorated engine or an engine with variable geometry, the running line moves as engine condition and/or inlet conditions change. In this type of engine model, using the Hot End Method would facilitate the programming, save memory space and computing time.

(6) Modelling flexibility is largely increased by 1-D searching technique; almost all the important operating parameters can be selected as the independent parameter. Thus the model is similar to the real engine, making it easier to relate to field operation.

The Hot End . "othod is initially derived from the thermodynamic model of a gas turbine engine; no niochanical parameters, such as speeds, are needed. Also because the generalized turbine characteristic is independent of sfeed, the method seems 
to have little dependence on turbomachinery features of the engine. No mechanical parameters of the engine can actually be simulated without component characteristic maps. This is the major shortcoming of this method. It exists as the method is created.

A generalized compressor characteristic map developed by Saravanamuttoo and Maclsaac (1983) has been used in the computational model of the LM-1600. This makes the model capable of predicting the mechanical performance. But because this compressor characteristic is introduced in graphic form, it also brings some graphic work into the program.

To create a good gas turbine model, it is necessary to include the engine's turbomachinery features. In doing this, if we can physically and mathematically explain the compressor speed lines, like we did for turbine, we may expect to gain some further knowledge.

It was interesting to find in the late stage of this study that the mathematical model used in the Hot End Method has some similar features to the one introduced by Cockshutt (1968). In that paper, Cockshutt analyzed the operation of a simple cycle one-spool turbojet engine by using a Logarithmic Differential Method. For a simple analysis, which means the specitic heat is assumed to be constant and fuel addition is negligible, the analytical model contains 6 equations and 7 unknowns that are the same as the one-spool jet engine model derived by the Hot End Method. Cockshutt also used the nozzle equation to simulate the turbine characteristic, but the choked condition was pre-assumed. Instead of using work compatibility and flow compatibility, Cockshu't used work compatibility and thermodynamic relations as the basic model equaticns, which are used to relate unknown temperatures to unknown pressures in the Hot End Method. By logarithmic differentiation, Cock. shutt's method gave good predictions on performance responses to small perturbations. The Hot End Method is mainly used for engine performance simulation rather than response analysis, so differentiation is not used. But if the Hot End modei is differentiated by the similar way, it could also function as a response predictor.

It is worth mentioning that the Hot End Method can also be used for aircraft engines, three-spool engines and engines with complex flow pattern. For aircraft engine, the altitude and Mach Number should be specified. For three-spool engine, the programmer will face 3 unknowi.s in 3 equations at the step (d), which is still 
not difficult. Mathematical models and sample calculations of some the engines had been done. For a gas turbine having more components and complicated flow pattern, such as intake nozzle, cooling air flow, blow off and etc, there will be more than 11 unknowns but also more than 10 available equations. If we can establish these additional models, the Hot End Method would still work. This question has been met in programming the computational model of the LM-1600. The programming work will be introduced in the next chapter. 


\section{Chapter 5}

\section{THE LM-1600 MODEL AND PROGRAM ANALYSIS}

The mathematical engine model introduced in Chapter 4 is derived for a simplified twin-spool gas turbine. Only the basic engine components are concerned. Real gas turbines would have more components. If cooling flow or fuel flow can not be ignored, and detailed performance prediction is required, new component and/or processing models should then be added to this basic model. The relation of $\mathrm{N}$ equations and $\mathrm{N}+1$ unknowns still exists, but the complexity of programming increases.

This question has been met when programming the computational model for the LM-1600. The name of this model is LM16. In this Chapter, the mathematical model of the LM-1600, the structure of LM16 and basic programming methods are introduced. Some special problems, such as convergency analysis and compressor modelling are discussed. The comparisons with field measurements are also presented. 


\subsection{Mathematical Model of The LM-1600}

In the initial mathematical model of a twin-spool gas generator introduced in Chapter 4, only basic engine components, compressors and turbines, are concerned. For a normal gas generator with no cooling air flow, no bleed air flow etc., this model is good enough. But if an engine has a complicated flow pattern, and more detailed predictions are required, this model should be modified by the real engine structure, to generate more information for the user.

The components of the LM- 1600 have been introduced in a previous chapter. The engine has a more complicated flow pattern then the "basic engine", so the basic mathematical model has to be modified.

\subsubsection{Flow Compatibility}

As mentioned previously, the LM-1600 is a twin-spool gas generator with cooled turbine blades and variable compressor geometries in the form of IGV, VSV and VBV. The general flow pattern of the LM-1600 is shown in Fig. 2. If the work load of the engine is lower than $95 \%$ of the design value, the VBV will open and part of the air flow between the LPC and the HPC will be blown off to atmosphere. Referring to the flow compatibility relations at the design point introduced in Chapter 3 , two modifications must be done to match the general flow pattern:

$$
\text { (1). } M_{1} \neq M_{2} \text {, but } M_{1}-M_{b}=M_{2} \text {; }
$$

$$
\text { (2). } M_{1}+M_{f} \neq M_{6} \text {, but } M_{2}+M_{f}=M_{6} \text {. }
$$

where $M_{b}$ is the flow rate of the air blown off between the LPC and the HPC.

\subsubsection{Governing Equations}

Referring to Fig. 2 and the governing equations in Chapter 4, the general governing equations of component matching of the LM-1600 should take the following form: 
Flow compatibility between the LPC and the HPC:

$$
\begin{aligned}
\frac{M_{2} \sqrt{T_{2}}}{P_{2}} & =\frac{M_{2}}{M_{1}} \frac{M_{1} \sqrt{T_{1}}}{P_{1}} \frac{P_{1}}{P_{2}} \sqrt{\frac{T_{2}}{T_{1}}} \\
& =R_{b} \frac{M_{1} \sqrt{T_{1}}}{P_{1}} \frac{P_{1}}{P_{2}} \sqrt{\frac{T_{2}}{T_{1}}}
\end{aligned}
$$

where $R_{b}=\frac{M_{2}}{M_{1}}$ is the Blow Off Coefficient.

Flow compatibility between the HPC and the HPT:

$$
\begin{aligned}
\frac{\left(M_{4}+m_{21}+m_{22}\right) \sqrt{T_{4}}}{P_{4}} & =\frac{\left(M_{4}+m_{21}+m_{22}\right)}{M_{1}} \frac{M_{1}}{M_{2}} \frac{M_{2} \sqrt{T_{2}}}{P_{2}} \frac{P_{2}}{P_{3}} \frac{P_{3}}{P_{4}} \sqrt{\frac{T_{4}}{T_{2}}} \\
& =\frac{1}{R_{1}} \frac{M_{1}}{M_{2}} \frac{M_{2} \sqrt{T_{2}}}{P_{2}} \frac{P_{2}}{P_{3}} \frac{P_{3}}{P_{4}} \sqrt{\frac{T_{4}}{T_{2}}}
\end{aligned}
$$

where $R_{1}=\frac{M_{1}}{\left(M_{1}+m_{21}+m_{22}\right)}$ is one of the Flow Coefficients.

Flow compatibility between the HPT and the LPT:

$$
\begin{aligned}
\frac{\left(M_{5}+m_{1}\right) \sqrt{T_{5}}}{P_{5}} & =\frac{\left(M_{5}+m_{1}\right)}{\left(M_{4}+m_{21}+m_{22}\right)} \frac{\left(M_{4}+m_{21}+m_{22}\right) \sqrt{T_{4}}}{P_{4}} \frac{P_{4}}{P_{5}} \sqrt{\frac{T_{5}}{T_{4}}} \\
& =R_{2} \frac{\left(M_{4}+m_{21}+m_{22}\right) \sqrt{T_{4}}}{P_{4}} \frac{P_{4}}{P_{5}} \sqrt{\frac{T_{5}}{T_{4}}}
\end{aligned}
$$

where $R_{2}=\frac{\left(M_{5}+m_{1}\right)}{\left(M_{1}+m_{21}+m_{23}\right)}=\frac{M_{6}}{\left(M_{1}+m_{21}+m_{22}\right)}$ is another Flow Coefficient.

Flow compatibility between the LPT and the Power Turbine:

$$
\frac{M_{6} \sqrt{T_{6}}}{P_{6}}=\frac{\left(M_{5}+m_{1}\right) \sqrt{T_{5}}}{P_{5}} \frac{P_{5}}{P_{6}} \sqrt{\frac{T_{6}}{T_{5}}}
$$

where $M_{6}=\Lambda_{5}^{\prime}+m_{1}$.

Work compatibility between the LPC and the LPT:

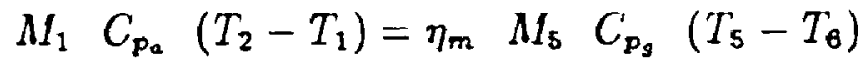


Work compatibility between the HPC and the HPT:

$$
\left(M_{2}-\frac{3}{7} m_{1}\right) C_{p_{4}}\left(T_{3}-T_{2}\right)=\eta_{m}\left(m_{21} C_{p_{4}} T_{3}+M_{4} C_{p_{s}} T_{4}-\left(M_{4}+m_{21}\right) C_{p_{s}} T_{5}\right)
$$

or

$$
\begin{aligned}
C_{p_{4}}\left(T_{3}-T_{2}\right)=\eta_{m} & {\left[\frac{m_{21}}{\left(M_{2}-\frac{3}{7} m_{1}\right)} C_{p_{4}} T_{3}+\frac{M_{4}}{\left(M_{2}-\frac{3}{7} m_{1}\right)} C_{p_{g}} T_{4}\right.} \\
& \left.-\frac{\left(M_{4}+m_{21}\right)}{\left(M_{2}-\frac{3}{7} m_{1}\right)} C_{p_{g}} T_{5}\right]
\end{aligned}
$$

or

$$
C_{p_{a}}\left(T_{3}-T_{2}\right)=\eta_{m}\left(R_{3} C_{p_{a}} T_{3}+R_{4} C_{p_{g}} T_{4}-\left(R_{3}+R_{4}\right) C_{p_{g}} T_{5}\right)
$$

where the term $\frac{3}{7} m_{1}$ is due to the LPT cooling air, $m_{1}$. Since it is drawn from the exit of the fourth stage of the HPC, it takes only about four sevenths of the total enthalpy raise in the seven-stage compressor. $R_{3}=\frac{m_{21}}{\left(M_{2}-\frac{3}{7} m_{1}\right)}$ and $R_{4}=\frac{M_{4}}{\left(M_{2}-\frac{3}{7} m_{1}\right)}$ are the last two Flow Coefficients.

\subsubsection{Generalized Turbine Model}

As mentioned in Chapter 4 , the generalized turbine characteristic can be expressed as a single curve. Assuming that the effective flow area and pressure ratio of a turbine are related to the maximum flow rate through the :urbine, which is the summation of main stream gas flow and the cooling air escaping from the tip and surface of the blades, the generalized turbine characteristic can then be written in the following form:

LPT:

$$
\frac{\left(M_{4}+m_{21}+m_{22}\right) \sqrt{T_{4}}}{P_{4}}=f_{n}\left(\frac{P_{4}}{P_{5}}, A_{h p l}\right)
$$

HPT:

$$
\frac{\left(M_{5}+m_{1}\right) \sqrt{T_{5}}}{P_{5}}=f_{n}\left(\frac{P_{5}}{P_{6}}, A_{l p l}\right)
$$

Power Turbine:

$$
\frac{M_{6} \sqrt{T_{6}}}{P_{6}}=F_{n}\left(\frac{P_{6}}{P_{7 s}}, A_{p l}\right)
$$


Since the effective flow areas have been found at the Design Point Calculation (DPC), the above equation can be re-written as:

$$
\begin{gathered}
\frac{\left(M_{4}+m_{21}+m_{22}\right) \sqrt{T_{4}}}{P_{4}}=f_{n}\left(P_{4} / P_{5}\right) \\
\frac{\left(M_{5}+m_{1}\right) \sqrt{T_{5}}}{P_{5}}=f_{n}\left(P_{5} / P_{6}\right) \\
\frac{M_{8} \sqrt{T_{6}}}{P_{6}}=F_{n}\left(P_{6} / P_{76}\right)
\end{gathered}
$$

The basic mathematical model of the LM- 1600 consists of the nine equations and the pressure equilibrium relation (Equation (4.10) in Chapter 4 ).

\subsection{Component Models}

Comparing the mathematical model of the LM-1600 with the initial one in Chapter 4 , it is found that five additional variables, $R_{1}, R_{2}, R_{3}, R_{4}$ and $R_{b}$, are introduced. These additional variables are induced by the blow off air flow, fuel flow addition and three cooling air flows. To solve this engine model, either new assumptions or specifications should be made, or new component models should be used. The latter one is the practice in program LM16.

\subsubsection{Blow off Air Flow Modelling}

The Blow off Air Manifolding and the VBV are safety protection devices to protect the compressors from surge. When the engine load is lower than the designed load, the pressure and the flow density at compressor exit drops and Mach Number rises. This increases the angle of attack to the blades at the rear stages and may cause blade stall, consequently the compressor may surge. To avoid this, the Bleed Valve opens and blows some air to atmosphere to lower the Mach Number at the rear of the compressor. In the LM-1600, the opening of the VBV is controlled by rotor 
speed and inlet temperature signals, i.e. the aero speed. From field measurements, it is apparent that the opening range of the VBV is quite wide.

In LM16, the bleed air flow, or VBV, is simulated by a valve model that can be written as:

$$
Q_{b}=C_{v} \sqrt{\frac{\Delta P}{\Delta P_{0}} \frac{\rho_{0}}{\rho}}
$$

where:

$Q_{b}$ - Volume flow rate of the blow off air;

$C_{v}$ - Flow coefficient of the valve, which is proportional to the opening of the valve;

$\Delta P-$ Operating pressure difference;

$\Delta P_{0}$ - Reference Pressure difference;

$\rho$ - Working fluid density;

$\rho_{0}$ - Reference fluid density.

Assuming the opening of the valve is proportional to the non-dimensional mass flow, or inlet Mach Number, of the LPC, then relating the non-d mass flow with the rotor rotational speed, the rate of air blown off can be estimated for every engine working condition.

\subsubsection{Fuel Flow and Combustion Process Modelling}

During a gas turbine combustion process, the compressed air from the HPC exit enters the combustor at low temperature; fuel is added, mixed with the air and burned; the hot gas leaves the combustor at high temperature. The combustion process raises the temperature, slightly increases the flow rate, causes some frictional pressure and momentum loss, and changes the stagnation enthalpy and gas properties of the flow.

In LM16, this process is simulated by fuel flow modelling, enthalpy and specific heat change and the assumption of constant pressure loss ratio. The fuel flow modelling is done by the same Energy Balance Equation used in the DPC. After the temperatures and enthalpies at the inlet and outlet of the combustor are determined, the fuel/air ratio can be found. Then the fuel addition can be calculated by 
the flow compatibility relations. The value of the specific heat of the working flow changes from air to gas after the combustion.

\subsubsection{Cooling Air Flow Modelling}

There are three cooling air flow passages for the blade cooling air of the HPT nozzle, the HPT rotor and the LPT. Theoretically, the flow rate in each passage is deteimined by the geometry of the passage, roughness of the passage, flow properties which are functions of pressure and temperature, state of the flow and the pressure differences between the inlet and the outlet of the passage.

For each flow passage, a fixed aero-geometry is assumed. If the flow properties and flow state are also assumed to keep unchanging, the frictional coefficient of the passage calculated from the DPC can then be used for all other operating conditions. Then the cooling air flow rate is mainly a function of the pressure difference. By determining the pressure difference of each passage, the cooling air flow rates can be estimated.

\subsubsection{Consideration of Inlet Guide Vanes and Variable Stator Vanes}

There is one IGV for the LPC and three V'SV stages for the HPC. When the engine is running at lower speeds, the IGV and VSV are rotated towards close, to protect the front stages of the LPC and the HPC from stalling and the rear stages from clioking When the engine is running at higher speeds, the IGV and VSV are rotated the other way. With the IGV and VSV clor Ing, the speed lines on the compressor maps are shifted to the bottom left; with them opening, the speed lines are shifted to the top right. So, the overall effects of IGV and VSV on the compressors are to enlarge the compressor maps along the running lines.

To simulate the effects, we can use enlarged compressor characteristics by assuming these variable geometry features had been built into the maps, or use normal compressor maps and shift the speed lines according to each individual running condition; or we can interpolate individual compressor maps of different geometry settings. The first method is relatively easy and has been used in LM16. 'To use the second method, it is necessary to simulate the speed lines of compressor char- 
acteristic. Simple mathematic expressions, such as polynomials, can be used for this purpose. But things tend to become complicated as these speed lines are being shifted. It was not felt that enough information on stage characteristics could be obtained to make the second method viable. The third method is obviously too large for a PC model and required information is also not available.

\subsection{Compressor Modelling}

The object of compressor modelling is to find compressor operating parameters which are pressure ratio, non-dimensional mass flow, efficiency, rotational speed and cleck the surge condition. Since pressure ratio and non-din.ensional mass flow are solved from the hot end method model and the speed is not initially ir.volved, compressor efficiency is primarily concerned in the modelling.

In the initial gas generator model (Chapter 4), it was assumed that the compressor efficiencies can be found by some means. In a real engine model, these efficiencies should be either found from the component characteristic maps, or estimated by analysis. In program LM16, the compressor efficiencies are found from a generalized compressor characteristic map recommended by Saravanamuttoo and Maclsaac (1983). The compressor efficiency estimation was also done during the course of this study. Both approaches are introduced in this section.

\subsubsection{The Generalized Compressor Characteristic}

In some sense, the mathematic model of a gas turbine is a thermodynamic model. The efficiency may be the only parameter in the model that partially reflects the aerodynamic performance of the machine. When the Hot End Method and the speed independent turbine characteristic are used, the compressors seem to be the thermodynamic mapping of turbines, 1.e. for every turbine operating point, there is a corresponding operating point on compressor map. Although the variation of mechanical speed is the way to realize the mapping, it does not appear in this thermodynamic model. So if the compressor characteristic is absent, prediction of speeds ss not possible. 
In LM16, a generalized compressor characteristic developed by Saravanamuttoo and Maclsaac (1983), has been used to simulate the performance of the LPC and the HPC. This generalized characteristic was derived from a number of known gas turbine compressors by relative scaling of the characteristic maps. The generalized characteristic is capable of handling the LM-1j00 compressors with pressure ratios of around 4 to 5 . This generalized compressor characteristic is expsessed in graphic form. The maps scaled up for the LPC and the HPC are shown in Fig. 17 and Fig. 18. In LM16, the surge line and speed lines are stored by polynomials, the efficiency contours are stored by finely divided straight lines.

In order to simulate the performance of a deteriorated engine and operation at different inlet conditions, the movement of the compressor running line has to be determined. Then the efficiency should be considered as the function of location of the working point on the map. In LM16, the efficiency is found by a simple method that is similar to polar coordinates and takes the centre of the highest efficiency contour as the origin. After the working point is located on the map, the compressor efficiency can be found by the polar angle, the length of polar axis and the efficiencies along the adjacent contou's.

After a Map Calculation, a series of working points on the LPC and the HPC; maps are found, allowing the operating lines to be plotted. The rotational speed functions can then be created by findir.; the cross points of the operating lines and the speed lines, and polynomial relation fitting. To check the surge condition is straightforward. Examples are shown in Fig. 17 and Fig. 18. In Fig. 17, the three operating lines represent the same engine with different blow off ratios. The first operating line or che top is zero blow off. It shows that surge will occur at low speed. The second line in the middle has the blow of ratio recommended by Farmer (1988) and field measurements, and is the one used in LM16. The third line is the operation with doubled blow off. Fig. 18 shows the associated operation lines on the HPC. Different blow off ratios have very little effect on the HPC operating line, but affect the spool speed relationship.

\subsubsection{Estimation of Compressor Efficiencies}

Because of the dificulty of obtaining the compressor characteristic maps, a systematic study has been carried out to analyze the effect of efficiency variation on the 
two compressor operating regions, to find a valid method to estimate the efficiencies when the compressor maps are not available. Another reason for this study is that compressor fouling and mechanical damage always change the characteristic and there is no eficient way to trace the change.

If a compressor characteristic map is not available, engine speeds may not be predictable, but the thermodynamic calculations can still be done as long as the efficiencies can be estimated.

When standard inlet conditions, or corrected parameters are considered, the running line, or operating line, of the coupressol is a single curve. So along the operatirg line, the efficiency and the speed should be able to be expressed as continuous functions of cumpressor operating parameters, pressure ratio or non-d mass how.

The efficiency estimation study for the LM-1600 has been done by the following steps. First, the efficiencies of the LPC and the HPC at the design poirt are found from the DPC. Then varying the efficiency of the HPC or the LPC separately from 80 to $105 \%$ of the designed value, the resuli1t.c cumpressor tunning lines re plotted o: Fig. 19 to Fig. 22.

When the LPC efficiency is assumed to be constant at the design value and the HPC efficiency varies, the possible operating region of the LPC is shown in Fig. 19, that is covered by the hypothetical running lines. Since the possible LPC operating lines are quite long, in order to keep the assumption, the designed LPC efficiency contour should be very large. But since only low efficiency contours are large ones, and the designed efficiency should be high, this LPC operating region is not likely possihie. Fig. 20 comes from the same assumption but for the HPC operating regions. Like Fig. 19, the dots in eac.. hypothetical running lines climb up as engine work load increases. It shows that as engine work load increases, the HPC efficiency shouid increase. Otherwise the HPC operating line will turn back from higher pressure ratio and higher non-d mass flow to lower ones. This is quite unusual.

Fig. 21 shows the possible LPC operating region when the HPC efficiency is ascumed constant at cesign value and the LPC efficiency varies. The region is very narrow. This means that the $L P C$ efficiency can vary dramatically along the operating line, but the shape of the operating line does not change much. From high 
efficiency around the design load to low efficiency at very low load, the operating line of the LPC is slightly convex. From the same assumption, the possible HP(' operating region is also quite narrow and concave, as shown in Fig. 22. For a short concave operating line on the HPC, the efficiency is easy to keep constant. For a long operating line on the LPC, the efficiency should be allowed to vary. These two operating regions seem to be logical.

The analysis concludes that if compressor characteristic maps are absent, onte simple and valid method is to assume that the HPC efficiency is constant at the design value, and fit the relationship between the LPC efficiency and the associated LPC parameters; then the modelling calculation can be carried out. This method will give reasonably accurate results at high load. The error may increase when the load is reduced. But the error should not be too large since the possible operating regions of the LPC and the HPC are relatively narrow.

In this analysis, it was also found that for a constant engine work output, as the LPC efficiency increases, the pressure ratio and non-d mass flow of the LPC increase", the pressure ratin and non-d mass flow of HPC decrease. As the HPC eficiency increases, the pressure ratio and non-d mass flow of the HPC increase; the pressure ratio of the LPC decreases, but the non-d mass flow of the LPC increases. It shows that as the efficiency of one compressor increases, the work load of the compressir will relatively increase and the load of the other compressor will relatively decrease.

\subsection{Program Structure}

\subsubsection{Overall Calculation Flow Path}

The overall program structure of LM16 is shown in Fig 23. The modelling calcu lation starts from the Design Point Calculation. Users have the right to jump over the DPC, but not the specification of opera.$g$ conditions. The Main Calculate..n, starting with an initial guess of $P_{6} / P_{70}$, is the core of this computational model The results of the Main Calculation will be given to the One-Dimensional Search Model to check whether the results meet the user's requirements or not. If not, $P_{6} / I_{7}$, should be inodified and fed back to the Mann Calculation. 
The flow path shown on Fig. 23 is generally valid for any Hot End Method program.

\subsubsection{The Main Calculation}

The Main Calculation flow path is shown in Fig. 24. Four types of additional models, two iterating loops and several coefficients are added into the original model. The calculating steps are explained as follows:

(a). The Main Calculation is started from the hot end. First, a guessed Power Turbine pressure ratio, $P_{6} / P_{7}$, is given to the program. By typical Hot End Method approach, the program will find a!t the pressure ratios and non-d mass flow rates for the turb:nes. In doing this, special care must be paid to the fuw rates. For each turbine, it should be able to swallow all the flows through it, which is the summation of main stream inlet hot gas and blade surface escaped cooling air. But for flow compatibility with upstream adjacent component, only the inlet hot gas is concerned. Since the flow rates of the cooling air are initially unknowns, design point values will be used for the first run of calculation and modified values will be used for later iterations

(b) The VBV model following the turbine models will calculate the Blow Off Coefficient $R_{b}$. Assumed $R_{b}$ will be used for the first run of calculation and calculated $R_{b}$ will be used for later runs.

(c). Then, the program will solve the work compatibility equations for turbine inlet temperature (TIT or $\left.T_{4}\right)$ and the LPC pressure ratio $\left(P_{2} / P_{1}\right)$. The compressor efficiencies used here are design values for the first run $c$ : calculation, and values from the generalized compressor maps for iterations

(d). As $T_{4}$ and $P_{2} / P_{1}$ have been found, all the temperatures, pressure ratios and non-d mass flow rates for the compressors can be solved by flow compatibility relations. This will locate the working points on the LPC and the HPC maps, hence the compressor efficiencies can be found. If the errors between the found efficiencies and previously used ones are larger than acceptable, modification to the efficiencies sliould be done. Then the renewed efficiencies will be fed back to step (c) and the calculation wili be repeated until the required accuracy is reached. This is the efficiency iterating loop.

(e). After the correct efficiencies, compressor working points in other words, 
are settled, the temperatures at all stations are fixed. The fuel/air ratio could be calculated by the combustion energy balance equation in the Combustor model

(f). Then the pressures at all stations can be determined. This allows the pressure difference between the inlet and outlet of each cooling air flow passage to be calculated, hence the cooling air flow rate. If the errors between the calculated cooling air flow rates and previously used ones are larger than acceptable, modified cooling air flow rates will be given back to step (a) and calculation will be repeated until the required accuracy is reached. This long drawn back is the cooling air iterating loop. It contains not only the cooling air flow modification, but also fued flow and bleed air flow modifications.

(g). From the accurate results of step (f), a!l the thermal parameters, and mechanical parameters if possible, of the engine can be found The results will be the true results associated with the prescribed $P_{6} / P_{7}$, If $P_{6} / P_{7}$, is the user specified parameter, or the calculation is part of the running line calculation, then the results need not to be further modified. But if some other parameters, such as TIT or work output is the user specified parameter, the results may not meet the user's requirement. Then $P_{6} / P_{7}$, will be automatically modified in the ( $)_{11}$ $D$ searching model, and the mann calculation will be repeated untill the required workıng condition is found.

\subsection{Convergence Analysis}

\subsubsection{Iterating Convergence}

When an engine is run at a steady state condition, there is only one possible workıng point. In other words, there is only one possible solution for a component matchng calculation; the result is a unique set of operating parameters So, theoretically ihr. iterating calculation in an engine model should be convergent unless the model is wrong.

If a model contains more than one iteration, the required iterating accuracies should be high. Otherwise, the errors will be amplified from one iteration to another In practice, several highly accurate iterations in a complicated engine model do not 
always succeed. In this kind of model, there are more than one equation, and each of them contains several variable parameters. If a wrong parameter is selected for the error analysis, or modification after each iteration is not done properly, the iteration may become an equal amplitude oscillation.

In LM16, the absolute difference between the calculated efficiencies and previously used ones is selected for the error analysis in the efficiency iteration. To select correct modification factors in step (d) of the Main Calculation is very important. The required accuracy of the efficiency iteration is less than $0.0002(0.02 \%)$ for both the LPC and HPC.

In the cooling air iteration, the summation of the absolute differences between the calculated cooling air flow rates and previously used ones is taken as the error. Although many flow parameters in this iteration seem to be acceptable for the error analysis, it is found that as cooling air flow rates converge, all the other flow parameters will converge. The accuracy required in this iteration is:

$$
\left|m_{1}^{\prime}-m_{1}\right|+\left|m_{21}^{\prime}-m_{21}\right|+\left|m_{22}^{\prime}-m_{22}\right|<0.0001 \mathrm{lb} / \mathrm{s}
$$

Where:

$m_{1}^{\prime}$ and $m_{1}$ - Calculated and previously used cooling air flow rate for LPT.

$m_{21}^{\prime}$ and $m_{21}$ - Calculated and previously used cooling air flow rate for HPT nozzle

$m_{22}^{\prime}$ and $m_{22}$ - Calculated and previously used cooling air flow rate for HPT rotor

In an engine model, the most sensituve parameters to iterating convergence may be the component work input and output. A divergent iteration will finally break the work compatibility relations.

\subsubsection{One-Dimensional Searching Convergence}

The convergence of One-D Searching is based on the monotonic increasing relationships between the Power Turbine pressure ratio, $P_{\mathbf{8}} / P_{70}$, and other thermodynamic parameters. For a normal simple cycle twin-spool gas generator, the relationships are shown on Table 5 and Fig. 16 of Chapter 4. When a new Hot End engine model is built, the first thing that should be done is to examine the monotonic relation- 
ships; i.e. to give a series of $P_{6} / P_{7}$, to a Hot End program, which does not contain the 1-D searching model, and analyze the results of the other parameters to see if the monotonic relationships exists or not. It is not necessary that all the monotonic relationships still exist when the flow pattern of an engine becomes complicated. But as long as a parameter has this relationship with $P_{8} / P_{7 a}$, the 1-D searching used for that parameter would always converge. Fortunately, all the important engine operating parameters do keep their monotonic relationships with $P_{6} / P_{7}$.

\subsection{Comparison with Field Measurements}

The comparisons between LM16's predictions and field measurements have been done. The measurements were taken at NOVA's Farrell Lake Compressor Station The altitude there is about 2700 feet. All the data had been corrected to IS() standard conditions. Comparisons are show nrom Fig. 25 to Fig. 29 where the scattered dots are measurement results and the lines are LM16's prediction.

Fig. 25 and 26 show that the predicted work output and rotational speed relations are very close to the measurements The predicted rotational speed relation between the two spools, shown in Fig. 27 , is also close to the measurements Fig 28 is the relation between the HPC exit static pressure and HP-spool speed It shows some error at high speed region This error may partially be caused by the inaccuracy of estimated HPC exit area and the Mach number, which could be corrected as more detailed information became available. The same problem also affects the prediction of gas generator EGT (or power turbine TIT which is used in field measurements) and HP-spool speed relation shown in Fig 29.

Fig 25 (1) to 29 (1) show the same comparison between full range Lil16 predsction and the best curve fits for the field measurements. For the reason mentioned at the end of 5.3.2, when the LPC is running in the highest efficiency contour, the increases of pressure ratio and non-d mass flow rate of the HPC with work output become very small. It makes the related working points on the HPC map very close to each other; hence the change of the HP-spool speed in this region is very small This causes a kink point on the speed relation diagram. As shown on Fig 27 (1), the kink has been detected by the measurement, and also predicted by the model. 
Fig. 30 is the comparison of predicted SFC with engine manufacturer's data. The agreement is very good.

Valid information on the LM-1600 is still limited. Considering both the inaccuracy of estimated factors in the model and the inaccuracy of the instrumentations, the predictions done by LMI6 are quite acceptable. 


\section{Chapter 6}

\section{THE MODEL APPLICATIONS}

\subsection{DPC and Model Engine Modification}

The LM16 program contans an interactive Design Point Calculation to show the DPC results to the user. The example output is shown in Table 6. It tells the user what the model engine initially looks like, how the components behave and shows engine overall performance and operating parameters at the design point. First, the user will be asked to specify two design point parameters, $\eta_{c \infty}$ and $T_{5}$ If the results derived from the previous DPC are given as the input, i.e. $\eta_{c \infty}$ equais $(19)$ and $T_{5}$ equals $1155 \mathrm{~K}$, the output will show the performance of normal LM-16in at the design point. By giving different values to the model, the user can actually modify the DPC, hence modify the model engine. But invalid values which will cause regative cooling air flow could not generate any results.

As explained in Chapter 3, the DPC is based on presently valid information This information locates the DPC solution within a small 2-D domain, but the final solution is still derived from an empirical assumption. To leave room for modification is necessary, since more information about the LM-1600 may becone available

This DPC modiñcation is also one way to trace engine degradation that always occurs in service As long as a degradation is tolerable, the engine will be kept in service. Gas turbine operators often find that engine test data after every waslung 


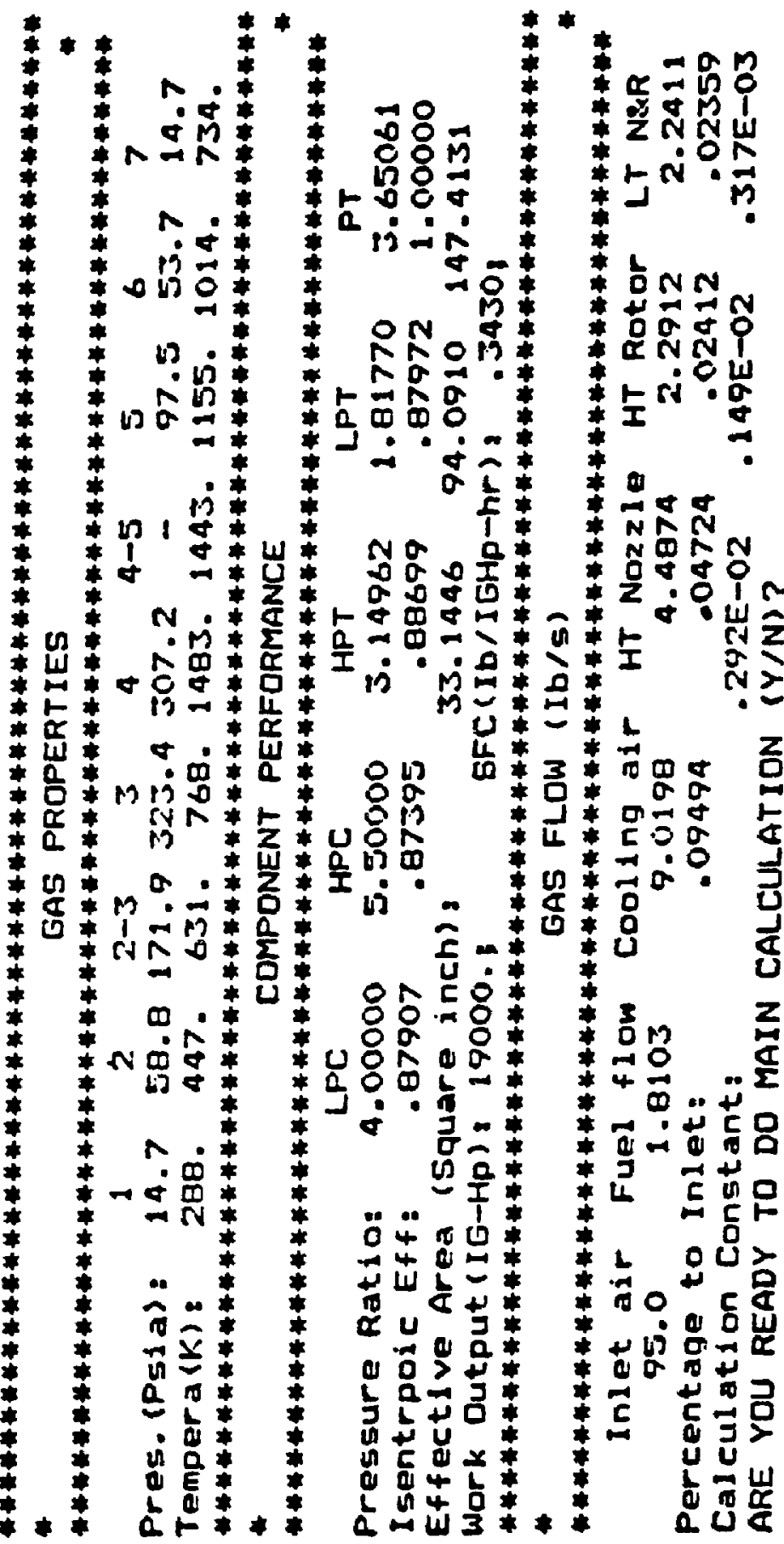

 
or overhaul diverge from the previous ones. To trace degradation in an engine model, a new design point calculation is usually required. To leave more design point parameters for the user's specification could increase the model's flexibility of tracing degradation, but would also increase the complexity of model application LM 16 only has two user specified design point parameters, some degradations can be traced by sophisticated use of these two.

\begin{tabular}{|c|c|c|c|c|}
\hline \multirow[t]{2}{*}{ No. } & \multirow{2}{*}{$\begin{array}{l}\text { Parameter } \\
\text { Selection }\end{array}$} & \multicolumn{3}{|c|}{ Valid Range } \\
\hline & & Low & Mid & High \\
\hline 1 & $W_{\text {out }}$ (IGHP) & 400 & 10000 & 20000 \\
\hline 2 & $T_{1}(\mathrm{~K})$ & 730 & 1230 & 1520 \\
\hline 3 & $T_{6}(\mathbf{K})$ & 540 & 840. & 1040 \\
\hline 4 & $T_{7}(\mathbf{K})$ & 520 & 655. & 750 \\
\hline 5 & Mass Flow $(\mathrm{lb} / \mathrm{s})$ & 38. & 81. & 96. \\
\hline 6 & $P_{2} / P_{1}$ & 1.3 & 3.1 & 41 \\
\hline 7 & $P_{3} / P_{2}$ & 3.6 & 5.1 & 56 \\
\hline 8 & $P_{9} / P_{1}$ & 4.8 & 16.2 & 226 \\
\hline 9 & Fuel Flow (lb/hr) & 610 & 3890 & 6880 \\
\hline 10 & LP-spool Speed (RPM) & 4800 & 11400 & 12500 \\
\hline 11 & $P_{6} / P_{7}$ & 1.15 & 2.68 & 3.74 \\
\hline \multicolumn{2}{|c|}{ Initial $P_{6} / P_{7}$} & \multicolumn{3}{|c|}{$1.5-2.5 \| 2.5-4.0$} \\
\hline
\end{tabular}

Table 7. Selection of The Fixed Parameter and Valid Range 


\subsection{Selection of The Fixed Parameter}

As explained in Chapter 4, we need one specified parameter to solve the mathematical engine model. In the real engine, this parameter is one of the controllable operating parameters. The operator can control the value of this parameter in order to achieve the requirec engine performance: In LM16, this parameter should be specified after the DPC. Then it is fixed in the modelling run. Eleven parameters that can be chosen as the fixed parameiei have been listed in Table 7. The meaningful ranges of these parameters are also given in this table.

After the user specifies the parameter, LM16 will find the corresponding engine working point by the one-dimensional searching. This is the basic way that the user controls the model engine. All information related to this working point will be given as model output. To check the real engine condition by comparing the modeling results with field measurements, the fixed parameter should be one of the best measured engine parameters.

\subsection{Specification of Running Conditions}

When the user runs LM16, some specifications have to be given to the model. In the case of the computer program, the specification services as model input; for the real engine, this is the description of the engine working environnent and engine condition.

\subsubsection{Inlet Conditions}

\section{Inlet Effect On Compressor Running Lines}

Generally, the performance of a gas turbine is the function of non-dimensional mass flow $\frac{M \sqrt{T}}{P}$ (axial Mach number) and non-dimensional speed $\frac{N}{\sqrt{T}}$ (tangential Mach number). If the gas turbine has fixed geometry, there is a single running line for each engine component in its full operating range. Theoretically the inlet conditions can only clange the location of working point along the running line, but not the location of the running line itself. For a variable geometry gas turbine, the 
iulet conditions do affect the location of component running lines.

For the LM-1600, the opening of the VBV is controlled by rotor mechanical speed and inlet temperature, i.e. aero speed of the engine. Assuming a constant work output is required, hot day will initially decrease the aero speed (although the mechanical speed increases. ) and open the VBV. More air will be blown off to atmosphere. The ratio of blow off rate to inlet flow rate increases. Some parameters are shown in Table 8.

\begin{tabular}{|c|c|c|c|c|c|}
\hline Parameters & \multicolumn{4}{|c|}{ Data } & Trend \\
\hline$T_{1}(K)$ & 248 & 268 & 288 & 308 & $\uparrow$ \\
\hline Mass $(l b / s)$ & 89.8 & 85.4 & 81.5 & 78.1 & 1 \\
\hline$M_{\text {fuel }}(l b / h r)$ & 3634 & 3760 & 3885 & 4010 & 1 \\
\hline OPR & 16.8 & 16.5 & 16.2 & 15.9 & $\downarrow$ \\
\hline $\operatorname{TIT}(K)$ & 1065 & 1148 & 1230 & 1313 & 1 \\
\hline$T_{6}(K)$ & 728 & 784 & 840 & 897 & 1 \\
\hline$T_{7}(K)$ & 564 & 610 & 657 & 704 & 1 \\
\hline$\frac{M_{1} \sqrt{T_{1}}}{P_{1}}$ & 96.2 & 95.1 & 94.1 & 93.3 & $u$ \\
\hline$\frac{N_{1}}{N_{\underline{T_{1}}}}$ & 678 & 674 & 670 & 667 & $\cup$ \\
\hline$M_{b}(l b / s)$ & 3.74 & 4.21 & 4.66 & 5.08 & 1 \\
\hline$\frac{M_{b}}{M_{0} g}(\%)$ & 4.17 & 4.94 & 5.71 & 6.50 & 1 \\
\hline $\operatorname{sfc}(l b / H p-h r)$ & .3646 & .3760 & .3885 & .4010 & 1 \\
\hline nehermal $(\%)$ & 38.1 & 36.8 & 35.6 & 34.5 & $\downarrow$ \\
\hline$N_{l}(\mathrm{rpm})$ & 10678 & 11033 & 11376 & 11708 & 1 \\
\hline$N_{h}(r p m)$ & 13447 & 13969 & 14474 & 14963 & $\uparrow$ \\
\hline
\end{tabular}

Table 8. Effect of Inlet Temperature $\left(W_{\text {out }}=10000\right.$ IGHP, $P_{1}=14.7$ Psia $)$ 
If constant mechanical speed is required, hot day will dramatically reduce the aero speed and increase the blow off air flow rate. The pressure ratio and the nondimensional mass flow rate will be largely reduced. Assuming a constant LP-spool mechanical speed $11300 \mathrm{RPM}$, which is about $90 \%$ of design speed, is required, the results are shown in Table 9.

\begin{tabular}{|c|c|c|c|c|c|}
\hline Parameters & \multicolumn{4}{|c|}{ Data } & Trend \\
\hline$T_{1}(K)$ & 248 & 268 & 288 & 308 & $\uparrow$ \\
\hline Mass $(l b / s)$ & 99.5 & 89.3 & 80.5 & 73.3 & $\downarrow$ \\
\hline$M_{\text {fuel }}(l b / h r)$ & 5243 & 4250 & 3795 & 3352 & $\downarrow$ \\
\hline OPR & 20.8 & 17.9 & 15.9 & 14.1 & $\downarrow$ \\
\hline $\operatorname{TIT}(K)$ & 1222 & 1191 & 1225 & 1247 & $T$ \\
\hline$T_{6}(K)$ & 836 & 814 & 837 & 852 & $\uparrow$ \\
\hline$T_{7}(K)$ & 613 & 620 & 658 & 690 & $\uparrow$ \\
\hline$\frac{M_{1} \sqrt{T_{i}^{-}}}{P_{1}}$ & 106.6 & 99.4 & 93.0 & 87.5 & $\downarrow$ \\
\hline$\frac{N_{1}}{\sqrt{T_{1}}}$ & 718 & 690 & 666 & 644 & $\downarrow$ \\
\hline$M_{b}(l b / s)$ & .46 & 2.87 & 5.02 & 6.81 & $\uparrow$ \\
\hline$\frac{M_{b}}{M_{0.8 s}}(\%)$ & .46 & 3.22 & 6.23 & 9.30 & $\uparrow$ \\
\hline sfc $(l b / H p \cdot h r)$ & .3360 & .3590 & .3950 & .4377 & $\uparrow$ \\
\hline$\eta_{\text {thermal }}(\mathscr{O})$ & 41.2 & 38.5 & 35.0 & 31.6 & $!$ \\
\hline$W_{\text {out }}(I G H P)$ & 15627 & 11840 & 9606 & 7657 & $\downarrow$ \\
\hline$N_{h}(r p m)$ & 13953 & 14115 & 14460 & 14741 & $\uparrow$ \\
\hline
\end{tabular}

Table 9. Effect of Inlet I'emperature $\left(N_{l}=11300 \mathrm{RPM}, P_{1}=14.7\right.$ Psia)

The inlet pressure also has some effect on the engine running lines. The altitudes 
of NOVA's booster stations, where the LM-1600's are installed, are usually about 3000 feet. The inlet pressure there is abcut 13 Psia, $11 \%$ less than sea level stand ard pressure. The low inlet pressure resuits in lower cornpressor output pressure and lower driving force of the coolant flow. So the cooling air flow rates of the LM-1600 at these spots should be lower than the ones at sea level. This effect is shown on Table 10 and 11.

\begin{tabular}{|c|c|c|c|c|c|c|}
\hline Parameters & \multicolumn{5}{|c|}{ Data } & Trend \\
\hline$P_{1}$ (Psia) & 14.7 & 14.3 & 14.0 & 13.5 & 13.0 & \rfloor \\
\hline Mass $(l b / s)$ & 90.5 & 88.5 & 87.1 & 84.5 & 82.2 & $\downarrow$ \\
\hline$M_{\text {fuel }}(l b / h r)$ & 5252 & 5234 & 5221 & 5197 & 5170 & $u$ \\
\hline OPR & 19.7 & 20.0 & 20.2 & 20.6 & 21.0 & 1 \\
\hline $\operatorname{TIT}(K)$ & 1351 & 1364 & 1375 & 1392 & 1409 & $i$ \\
\hline$\frac{M_{1} \sqrt{T_{1}}}{P_{1}}$ & 104.4 & 105.1 & 105.6 & 106.4 & 107.3 & $\uparrow$ \\
\hline$N_{l}(r p m)$ & 12033 & 12074 & 12106 & 12162 & 12222 & 1 \\
\hline$N_{h}(r p m)$ & 14861 & 14923 & 14971 & 15060 & 15156 & 1 \\
\hline sfc $(l b / H p-h r)$ & .3501 & .3490 & .3481 & $.346 j$ & .3447 & $u$ \\
\hline$\eta_{\text {thermal }}(\%)$ & 39.5 & 39.6 & 39.7 & $j 9.9$ & 40.1 & $n$ \\
\hline$m_{1}(l b / s)$ & 1.97 & 1.89 & 1.84 & 1.74 & 1.65 & 1 \\
\hline$m_{21}(l b / s)$ & 3.83 & 3.69 & 3.59 & 3.43 & 3.27 & $\downarrow$ \\
\hline$m_{22}(l b / s)$ & 1.95 & 1.89 & 1.83 & 1.75 & 1.67 & 1 \\
\hline$M_{c}(16 / s)$ & 7.75 & 7.47 & 7.26 & 6.92 & 6.59 & $\downarrow$ \\
\hline$\frac{M_{c}}{M 0 s}(\%)$ & 8.56 & 8.44 & 8.34 & 8.18 & 8.02 & $!$ \\
\hline
\end{tabular}

Table 10. Effect of Inlct Pressure

$$
\left(W_{\text {out }}=15000 \mathrm{IGHP}, T_{1}=288 \mathrm{~K}\right)
$$


The effect of inlet conditions on compressor running lines is shown on Fig. 31 and 32. Although the lines in these figures are almost indistinguishable, it still can be seen that low inlet temperature and low altitude (such as $T_{1}=243 \mathrm{~K}$ and $P_{1}=14.7 P$ sia ) bring the LPC running line up, and high temperature and high altitude (such as $T_{1}=303 \mathrm{~K}$ and $P_{1}=13.0 \mathrm{Psia}$ ) bring the running line down.

\begin{tabular}{|c|c|c|c|c|c|c|}
\hline Parameters & & & Data & & & Trend \\
\hline$P_{1}$ (Psia) & 14.7 & 14.3 & 14.0 & 13.5 & 13.0 & 1 \\
\hline Mass (lb/s) & 95.0 & 92.5 & 90.6 & 87.4 & 84.1 & $\downarrow$ \\
\hline$M_{\text {fuel }}(\mathrm{Jb} / \mathrm{hr})$ & 6613 & 6414 & 6249 & 5975 & 5706 & $\downarrow$ \\
\hline OPR & 22.1 & 221 & 22.1 & 22.0 & 22.0 & $\cong$ \\
\hline TIT (K) & 1496 & 1492 & 1488 & 1480 & 1472 & $\downarrow$ \\
\hline$\frac{M_{1} \sqrt{T_{1}}}{S_{1}}$ & 109.7 & 1098 & 109.8 & 109.8 & 109.8 & $\simeq$ \\
\hline$W_{\text {out }}(\mathrm{IGHP})$ & 19239 & 18675 & 18205 & 17423 & 16652 & $\downarrow$ \\
\hline$N_{h}(\mathrm{rpm})$ & 15240 & 15269 & 15286 & 15313 & 15339 & $\uparrow$ \\
\hline sfc $(\mathrm{lb} / \mathrm{Hp}-\mathrm{hr})$ & .3437 & .3434 & .3433 & .3430 & .3426 & $\cup$ \\
\hline Dihermal $(\%)$ & 40.2 & 40.3 & 40.3 & 403 & 404 & $n$ \\
\hline$m_{1}(1 \mathrm{~b} / \mathrm{s})$ & 2.25 & 2.13 & 2.04 & 1.89 & 1.75 & $\downarrow$ \\
\hline$m_{21}(\mathrm{lb} / \mathrm{s})$ & 4.51 & 4.27 & 4.09 & 3.80 & 3.51 & $\downarrow$ \\
\hline$m_{22}(\mathrm{lb} / \mathrm{s})$ & 2.30 & 2.18 & 2.09 & 1.94 & 1.79 & $\downarrow$ \\
\hline$M_{c}(\mathrm{lb} / \mathrm{s})$ & 9.06 & 8.58 & 8.22 & 7.63 & 7.06 & $\downarrow$ \\
\hline$M_{0}(T)$ & 9.54 & 9.28 & 9.01 & 873 & 8.39 & $\downarrow$ \\
\hline
\end{tabular}

Table 11. Effect of Inlet Pressure

$\left(N_{l}=12400 \mathrm{RPM}, T_{2}=288 \mathrm{~K}\right)$ 


\section{Inlet Effect On Engine Overall Performance}

Sone the effects of inlet conditions on the engine overall performance have been previously disiussed. It can be seen from Table 8 that if the work output is kept constant, increase of inlet temperature will slightly decrease the axial and tangential Mach numbers tecause of the variable geometry. Mass flow drops and the TIT increases dramatically. Engine performance, by considering the thermal efficiency, tends to be worse. If the LP-spool mechanical speed is kept constant, as shown on Table 9, the increase of the inlet temperature will cause large drops of the axial and tangential Mach numbers. The performance degrades even faster.

Table 10 shows when the work output is kept constant, the increase of altitude, or decrease of $P_{1}$, will cause the increase of the axial and tangential Mach numbers Though both mass flow and fuel flow drop, the combined effect is the TIT rises and thermal efficiency slightly increases. This does not mean the increase of altitude has favourable effect on engine performance: instead, as TIT is kept at the limit, the work output goes down as altitude rises

From the same altitude argument, if the LP-spool speed is kept constant instead of the work output, the effect is shown in Table 11. The tangential Mach number m this case is constant The axial Mach number should be constant if the engine liad fixed geometry. So, all the parameter variations on this table are purely caused by the $\mathrm{VBV}$ and the change of cooling air flow rates.

\section{To Evaluate The Inlet Effect}

Although gas turbines are operated at different altitude and different weather conditions, field measurement for industrial gas turbines are usually corrected to ISO, or sea level, standard condition ( the ambient temperature $\left(T_{1}\right)$ is $288 \mathrm{~K}$, the ambient pressure $\left(P_{1}\right)$ is 14.7 Psia.). But to the field operator, the actial engine parameters, not the correcied ones, are visualized. LM16 gives the option to the user At the begining of the program, the user will be asked to snecify the inlet conditions. i.e. the values of $P_{1}$ and $T_{1}$. The specified conditions are not necessarily coincident with ISO standard condition; and the rodel will predict the actual performance parameters at that inlet conditions

There are two ways to evaluate the engine performance with the effects of the inler conditions. The user can correct the measured performarce parameters to IS() standard conditions and run the model at ISO condition, to compare the calculated 
results with the corrected parameters; or he can run the model engine at actual inlet conditions and compare the calculated results with uncorrected measurement parameters that the operitior would read at the field.

\subsubsection{Deteriorated Engine Conditions}

\section{Simulation of Deteriorated Engine}

Evaluation of the effects of a deteriorated engine is done by simulating expected engine faults and incorporating them into the model. This can be applied in three ways.

1. It gives the uses an opportunity to gain some insight into the engine degradation. In LM16, this is done by specifying a certain type of deterioration to the model, and comparing the results with those of a normal engine.

2. It can be used to develop fault matrices, helping the operator to detect possible engine problems. If the trend of the measured operating parameters varying from the baselines coincides with a trend of one of the fault matrices, the operator may deduce that type of engine damage has occurred.

3. The simulation can be used to trace a tolerable engine degradation. Since component degradations are refiected by the variations of component efficiencies, flow areas, flow rates and etc, varying these parameters continuously and matching the model engine with the real one is another way to trace the degraded engine. Instead of trend analysis, this is a quantitative analysis.

For the gas turbines used for pipeline application, Williams (1981) listed the most common problems in order of frequency as follows:

(i) Compressor efficiency;

(ii) Compressor mass flow;

(iii) Leakage (overboard);

(iv) Leakage (internal),

(v) HP turbine efficiency;

(vi) LP turbine efficiency;

(vii) HP turbine flow function;

(viii) LP turbine flow function.

Some of these problems have been simulated in LM16 for trend inalysis:

). LPC Mechanical Damage: simulated by $5 \%$ drop of LFC isentropic efficiency. 
2). Compressor Excessive Seal Leakage: simulated by $3 \%$ over-board leakage flow at the HPC exit.

3). HPT Mechanical Damage: simulated by $5 \%$ drop of HPT isentropic efficiency.

4). HPT Erosion: simulated by $2 \%$ increase of HPT flow area

5). LPT E'rosion: simulated by $2 \%$ increase of LPT flow area

At the begining of LM16, the user will be asked which deterioration wanted to be simulated.

\section{Deterioration Effect On Engine Overall Performance}

The evaluation of deterioration is done by comparing the deteriorated engme performance with that of normal engine. The comparison is meaningful only when one operating parameter is common in the two engines, i.e. this parameter is kept the same in both operations. In practice, this parameter should be ne of the controllable parameters that can be independently changed by the operator

When work output is chosen as the independent variable, the effects of deter 1 . oration on engine periormance are shown from Fig 33 to 37 and Table 12. Whe'n the LP-spool speed is taken as the independent variable, the effects are shown from Fig. 38 to 42 and Table 13. The units used in Fig. 33 to 42 are same as the units in Table 12 and 13 . Snice the trends of the parameter varying may be different from high load or high speed to low load or low speed, three different loads and speeds have been examined Fault matrices and trend analysis shown in Table 16 and 17 will be discussed later.

The variations of some important parameters in Fig. 33 to 42 shou that:

a. Work output as independent variable:

- Compressor mechanical damage will cause TIT, fuel flow and HP-speed increase; OPR and LP-spool speed decrease (Fig. 33 ).

- Compressor excessive leakage will cause TIT, fuel flow, LP and IIP-speed increase; and OPR decrease ( Fig. 34 ).

- HPT mechanical damage will cause OPR, TIT and fuel flow increase, as well as LP and HP-speed decrease ( Fig 35 ).

- HPT erosion will decrease OPR and HP-speed. TIT slightly decreases. Fuel flow and LP-speed do not change significantly ( Fig. 36 ). 


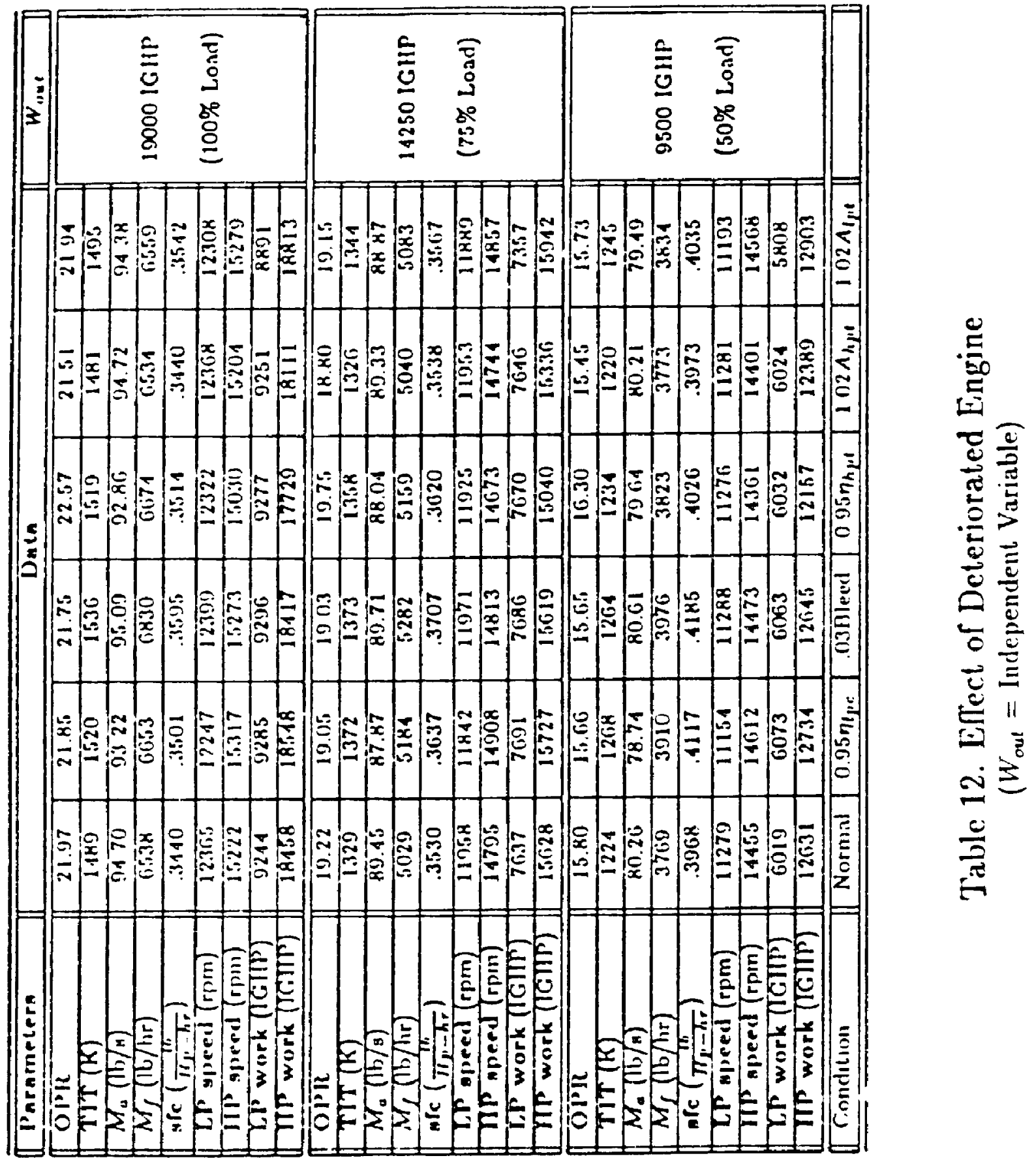




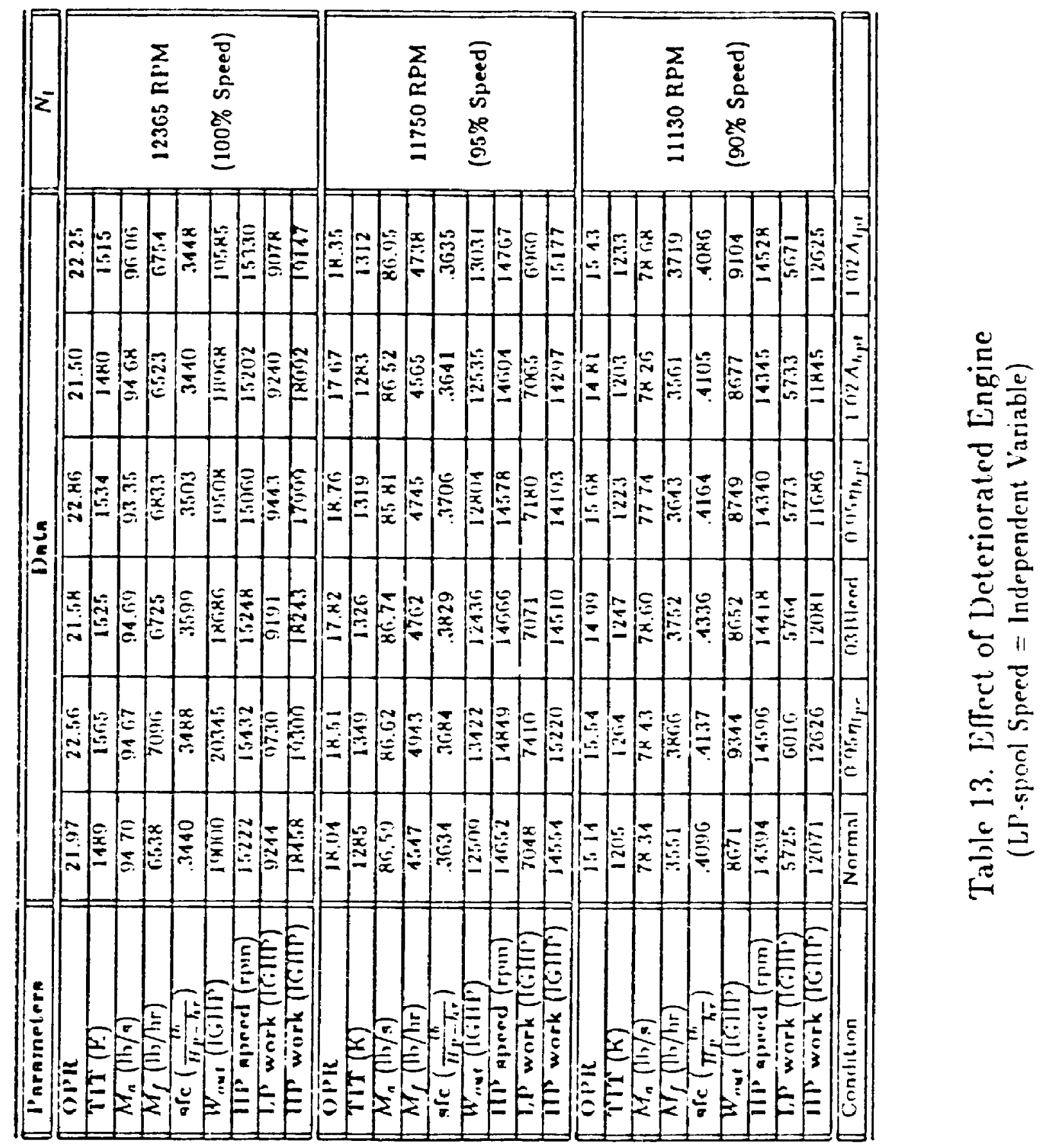


- LPT erosion will increase TIT and HP-speed, and will decrease LP-speed. Fuel flow increases slightly. OPR does not have significant change ( Fig. 37 ).

b. ¿P-spool speed as independent variable:

- Compressor mechanical damage will increase OPR, fuel flow and TIT; hence causing increase of work output ( Fig. 38 ).

- Compressor excessive leakage will increase TIT and fuel flow, and decrease OPR and work output ( Fig. 39).

- HPT mechanical damage will increase OPR, fuel flow and TIT; work output will s!ightly increase, especially at high speed ( Fig. 40 ).

- HPT erosion will decrease OPR; slightly TIT. Fuel flow and work output do not show significant change ( Fig. 41 ).

- LPT erosion will increase OPR, TIT, fuel flow and work output (Fig. 42).

\section{Deterioration Effect On Compressor Running Lines}

The effects of deterioration on compressor running lines are shown in Fig. 43 and 44. Within the specified degree of deteriorations, it shows:

a. In Fig. 43, HPT mechanical damage brings the LPC running line up; LPT erosion brings the line down; compressor mechanical damage, compressor excessive leakage and HPC erosion do not have significant effect on the line.

b. In Fig. 44, HPT mechanical damage brings the HPC running line up; compressor excessive leakage, LPT and HPT erosion bring the line down; compressor mechanical damage does not have significant effect on the line.

\subsection{The Model Outputs}

There are two types of outputs generated by LM16, the table outputs and graphic outputs.

\subsubsection{Table Outputs}

The table outputs are given on the screen at the end of the modelling calculation. If the DPC is wanted by the user, the DPC results will be listed on the first table; 


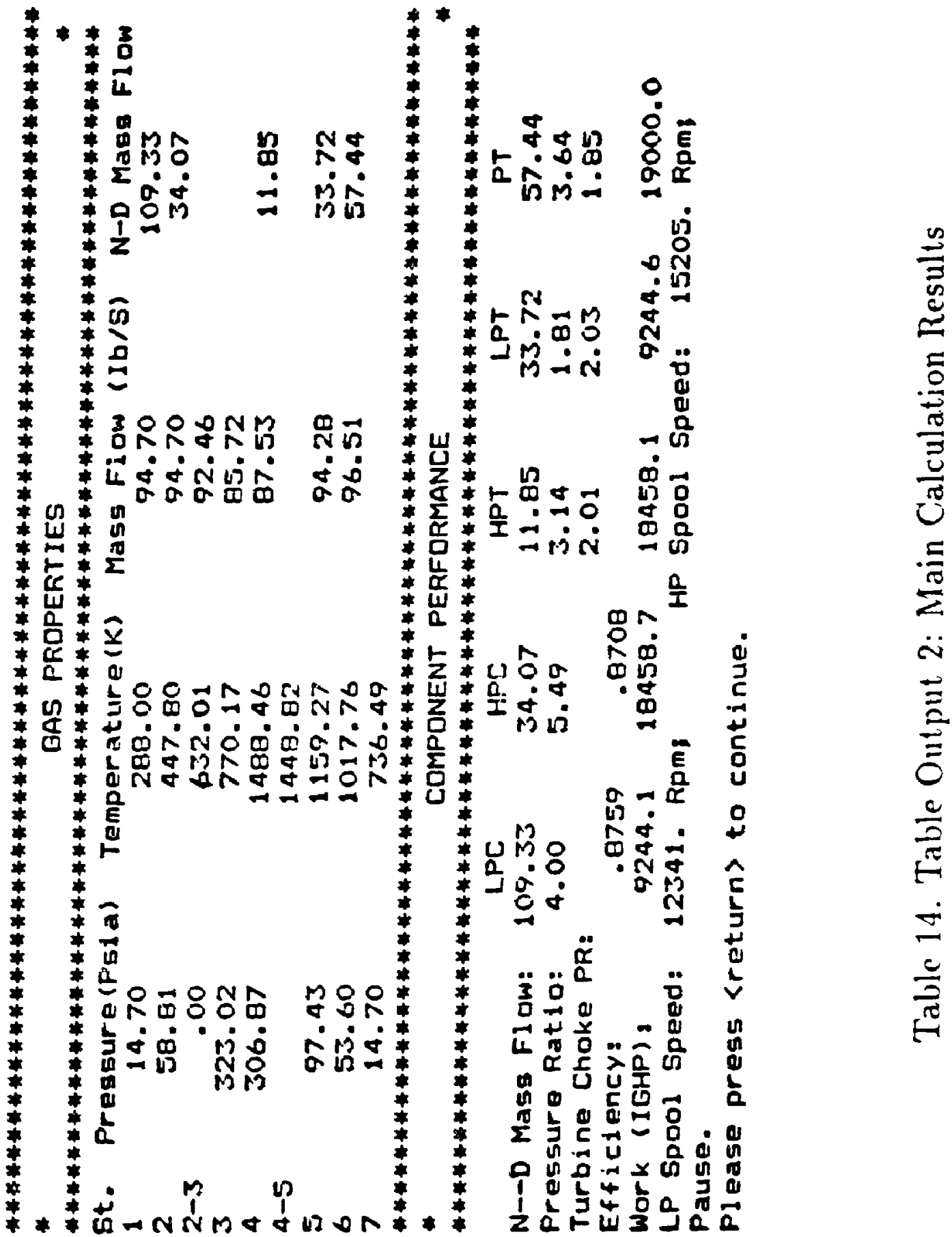




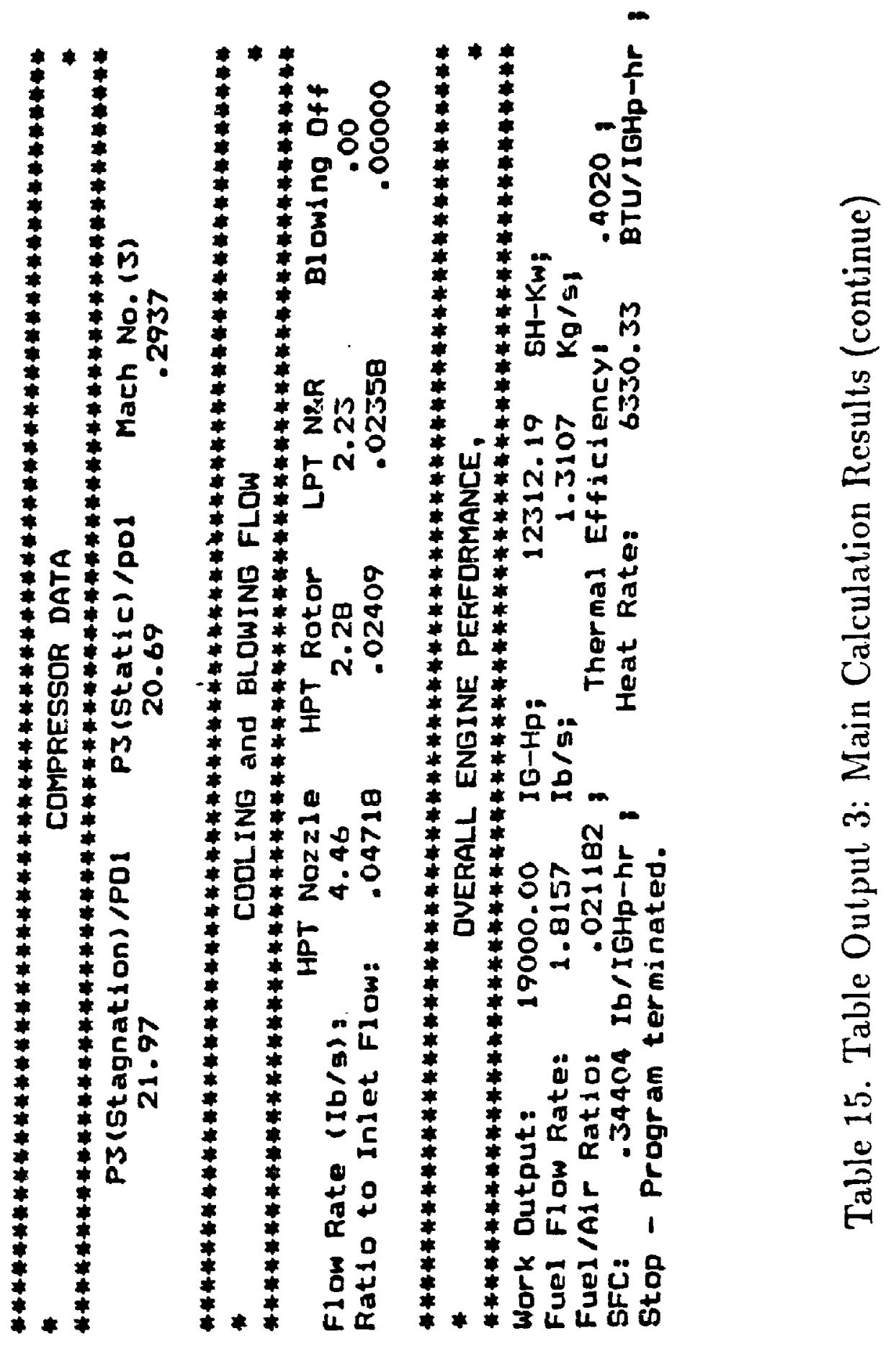


refer to Table 6. The Main Calculation results are given on screen by the second and the third tables; examples are shown in Table 14 and 15.

The Main Calculation results are divided into five columns:

1). Gas Properties: As the previously defined station numbering system, the pressure, temperature and non-dimensional mass flow at each station are given in the first column.

2). Component Performance: The major component pelformance parameters, pressure ratios and non-d mass flows, are shown in the second column. The possible critical pressure ratios of turbine choking are given as references. The work load split between the LP-spool and the HP-spool is also given. By analyzing the variation of the load distributions in different running conditions, the user can get some physical insight of the engine. Another application of this work load information is to clieck the convergency of the iteration in the model. Since a turbine's work output should be equal to the compressor's work input in the same spool, a large difference between the two is usually caused by the failure of iterating convergence. This kind of failures have been eliminated in LM16.

3). Compressor Data: According to the field instrumentation, the pressure measured at the exit of the HPC is a static pressure. To couple the model with the measurement, two kinds of OPR, static and stagnation, and the Mach number at the exit have been calculated and listed in column three. The calculation is based on an estimated flow area of the exit. Modification for more accurate results can be done by using field test data.

4). Cooling and Blown Off Flow: Cooling air flow rates and blown off air flow rate are listed in the fourth column. The ratios of these flows to the inlet mass flow are also calculated.

5). Overall Engine Per ormance: The fifth column shows the overall engine behaviour, i.e. the work output, fuel flow, sfc, thermal efficiency and heat rate.

To evaluate these data, an operator can find whether the engine works properly or not. By comparing these data with measured parameters, the possible engine or instrument errors can be roughly detected. 


\subsubsection{Mapping Calculation}

There are 12 graphic outputs corresponding to a Mapping Calculation. The purpose of this calculation is to give the user a graphic view of the engine operation. By comparing compressor running lines and other opezating parameters with their baselines of normal engine, the user can gain some better understanding about the engine operation and the phenomenon of deterioration.

The Mapping Calculation is done by specifying the deteriorated engine condition: (or non-standard inlet conditions) and choosing the Mapping Calculation. The graphic outputs contain:

1). LPC Running Line;

2). HPC Running Line;

3). LP-spool Mechanical Speed vs. HP-spool Mechanical Speed;

4). LP-spool Mechanical Speed vs. LPC Pressure Ratio;

5). HP-spool Mechanical Speed vs. HPC Pressure Ratio;

6). OPR (static) vs. HP-spool Mechanical Speed;

7). HP-spool Mechanical Speed vs. Work Output;

8). LP-spool Mechanical Speed vs. Work Output;

9). Compressor Efficiencies vs. Work Output;

10). Gas Generator EGT vs. HP-spool Mechanical Speed;

11). Mass Flow Ratio, $\frac{M_{2}}{M_{3}}$, vs. LP-spool Mechanical Speed;

12). SFC vs. Work Output.

Examples of the Graphic Outputs are shown from Fig. 45 to 56 . There are two lines plotted on each graph, the baseline of normal engine at ISO standard inlet conditions, and deteriorated engine working line based on user's specification. If user specifies different inlet conditions instead of engine deterioration, the comparison will be same as the one introduced in 5.2.1. If user specifies both non-standard inlet conditions and deteriorated engine, the graphic outputs will show the combined effect. 


\subsection{Coupling with Field Operation}

\subsubsection{Fault Matrices}

Fault matrix analysis is an efficient method used by gas turbine users. It is a trend analysis method. By comparing operating parameters with their baseline values, the trend of the parameter variation can be determined. To compare the trend with a set of pre-defined fault matrices, the possible engine problem can be found. A useful practice of LM16 is to establish the fault matrices for different deteriorations.

Different independent variables have different trends of fault matrices. Although almost any thermodynamic parameters and some mechanical parameters of an engine could be chosen as the independent variable, only those directly measured parameters can be practically used. Eleven parameters can be used to run LM16, shown in Table 7 . The possible independent variables, such as Work Output, Rotor Speed, Fuel Flow, OPR, Mass Fluw and EGT are covered in these parameters. Specifying an independent variable and $\mathrm{d}^{\mathrm{N}}$ eriorated engine condition to run the model, and comparing the results with those of normal engine, one can create the fault matrix of that deterioration under the predefined independent variable. Examples are shown on Table 16 and 17 by re-treating the data of Tatle 12 and 13, where:

$\uparrow$ - increasing trend,

$\downarrow$ - decreasing trend,

$n-$ slightly increasing,

$U$ - slightly decreasing, and

$\cong-$ almost no change.

These tables can be used in the field, and similar fault matrices of different independent variables can be created by the user if required. 


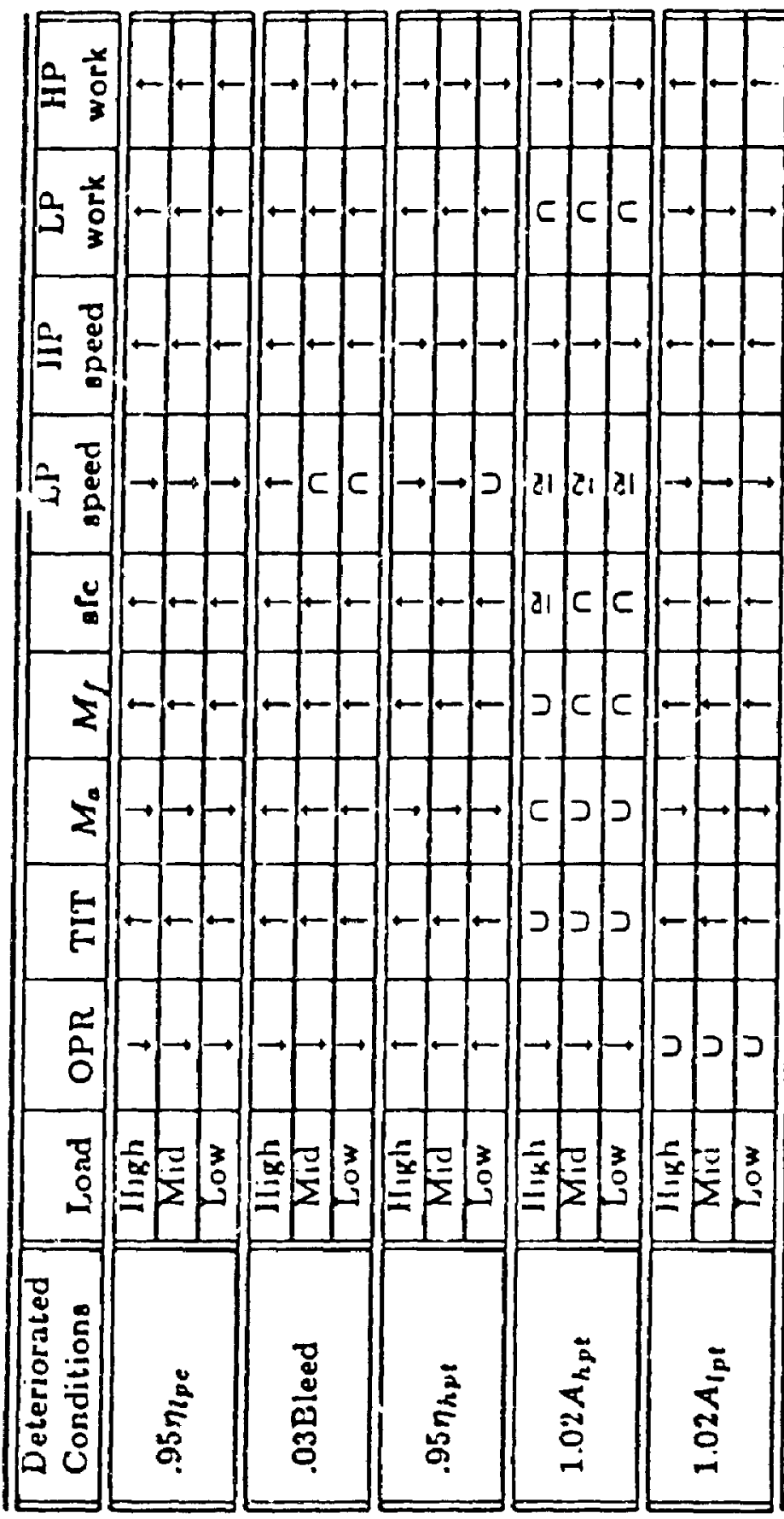

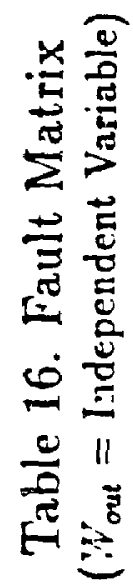




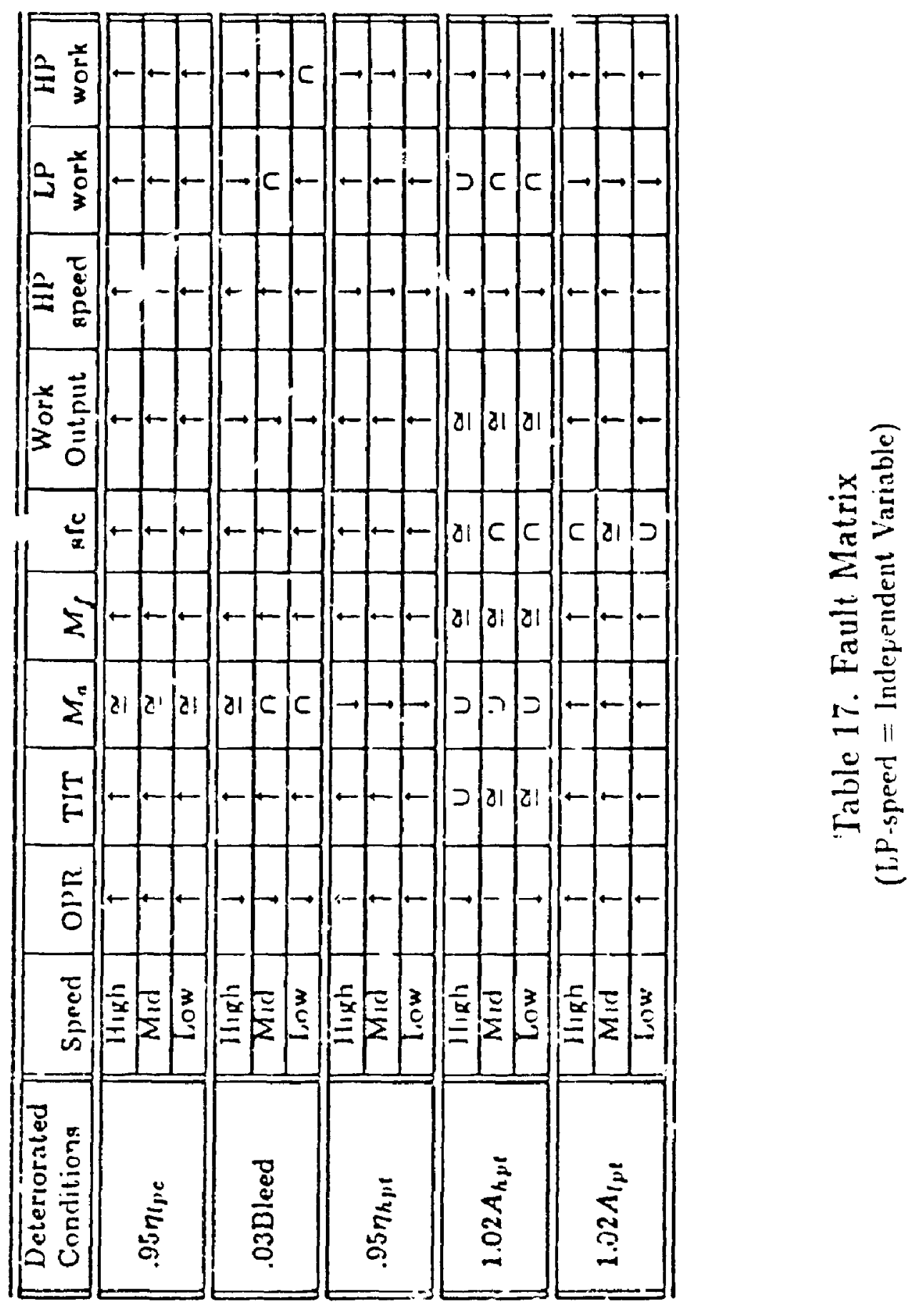




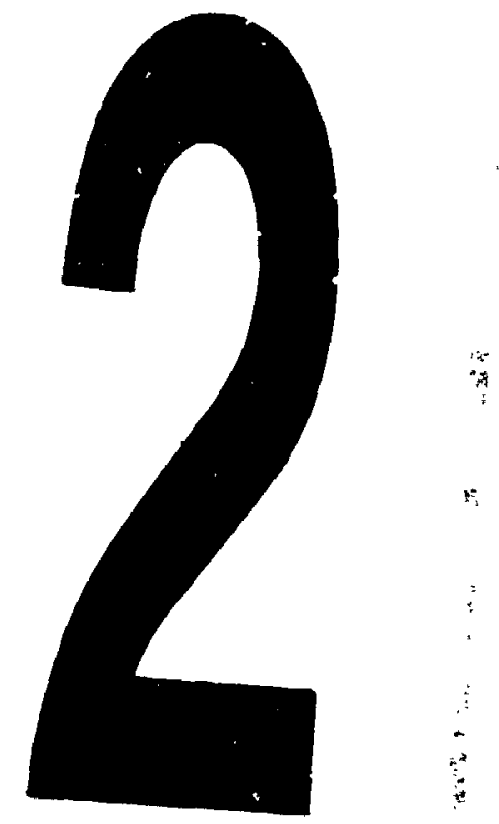

PM-1 31\%"X4" PHOTOGRAPHIC MICROCOPY TARGET : NOS 1010a ANSI/ISO \#2 EQUIVALENT

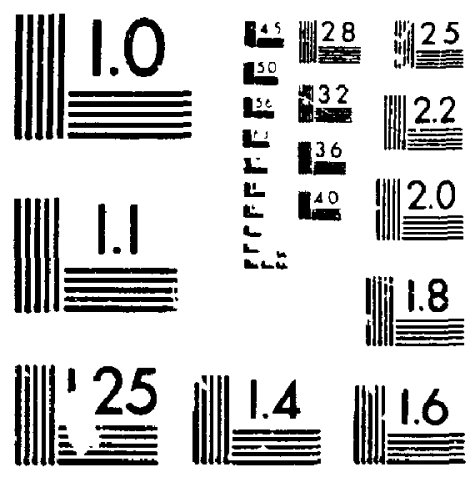




\subsubsection{Fault Detection}

For an Engine Health Monitoring system, it is important to ensure the measure. ment accuracy. As instrumentation error appears in the system, the interpretation of engine healthy status would not be correct any more. Since several gas turbine users have found the interference of instrumentation errors in their EHM system, it is reasonable to require the system to have the capability of validating measurement and detecting possible engine and/or instrumentation faults. A data validation package based on comparison of measured and GPA calculated parameters with pre-established baseline values has been introduced by Agrawal, Maclsaac and Saravanamuttoo (1979). As an on-site performance simulation model, LM16 could also be used for a similar purpose. Although it is certainly impossible to detect all of the engine and instrumentation faults by simple sofiware, it can still give field operator some valid reference indicating what may occur in the engine and instrumentation system. This is especially viluable to pipeline gas turbine users, where the booster stations are usually hundreds of miles away from the control centre; the feed back of measurement analysis from the centre to local stations could be considered very slow,

When tired for data validating, it is a preliminary requirement that the model should be able to trace engine degradation. This could be done by the methods introduced earlier. Fault matrices of common engine deteriorations based on all the measured pararneters should be pre-established. User's good knowledge of instrumentation would be helpful for analysis. The best or the second besi neasured parameter should always be the first clioice for the model engine independent parameter.

As daily operation, the user can run the model with the best measured parameter and normal engine specification, and compare the modelling results with the measurement. Analysis can be carried out by the following steps:

(?) If all the calculated parameters match with the measured ones, healthy engine and normal instrumentation are concluded.

(2) If only one parameter does not match but all others do, it is fair to say the instrument may have some error.

(3) If some parameters do not match but some do, the user should check the preestablished fault matrices. First, he should find a matrix which has the similar trend 
of parameter variation as the measurement; then take the second well measured parameter to run the model again. If the fault matrix of the second parameter indicates the same type of engine deterioration, the engine fault is confirmed. If the user can not find a fault matrix that could describe the measurement deviation, or he can not find the second matrix which indicates the same engine fault as the first one, then an uncommon engine fault or combined engine and instrumentation fault is likely. Changing the model engine independent parameter and running the inodel with deteriorated engine specifications might give some hints of the fault to the user.

(4) If all the calculated parameters do not match with the measurement except the independen: parameter, the user should re-run the model with the second well measured parameter since the error may be caused by the instrument fault of the first parameter.

In this analysis, compressor exit pressure, gas generator exit pressire and temperature, fuel flow and rotor speeds should be considered as important engine parameters and have priority over others. And since pressure and rotor speeds can be more easily and accuraiely measured than temperature, any mis-matches of pressure and rotational speed parameters more likely represent engine faults, and any mis-matching of temperature parameters is more likely to be instrumentation faults. Experience on both the real engine and the model is necessary for this analysis.

\subsubsection{Other Functions}

1. For new users of the LM-1600, the model LM16 can be used as a training tool. Since some operating conditions may seldom be seen in daily operation, the previous experience might only be obtained by operating the model engine LM16.

2. LM16 can be used as operating reference. In real engine operation, controllable parameters are limited. For example, if fuel flow is the only controllable parameter, in order to achieve certain work output, an operator should adjust the fuel throttle. Since the operator does not know the relationship between the work output and the fuel flow, he can first set the required work output value to LM16. The model will tell what is the approximate fuel flow rate needed for this work output. 
3. As a long distance energy shipper, a pipeline usually crosses several states and provinces with different geographic and weather conditions. With the function of evaluating the fuel-load relation at any inlet conditions, LM16 could be used in the pipeline control centre to help scheduling the whole line operation and evaluating the fuel economy.

4. For changeable climatic conditions, LM16 can be used to forecast the operation and performance of the engine according to weather forecast.

\subsection{Hardware Requirement}

LMi6 is a portable PC model and can be run at any regular PC. All the functions of LM16 are stored in two PC floppy discs. The Disc (1) is a calculating disc and contains the execution file and most of the data files. The Disc (2) is a graphic disc and contains all the plotting files and some graphic data files. If graphics are not wanted by the user, Disc (2) is not necessary.

The Table Output will be shown on the screen or printed out through a printer. This equipment has already been installed in NOVA's booster stations for health monitoring purpose. Data required by LM16 can be collected mailually or through the data acquisition system, which should not make a significant difference to the results. The only required new equipment is a plotter for the graphic outputs if the user not only wants to see the graphics on screen but also have a hard copy.

The average running time for each modelling case is about 1.5 minutes on a $\mathrm{XT}$ machine. The Mapping Calculation takes about 20 to 25 minutes. Any nonexperienced workers can operate LM16 with a little training in a very short period of time. 


\section{Chapter 7}

\section{RECOMMENDATIONS FOR FURTHER WORK AND CONCLUSIONS}

\subsection{Research of Component Mathematical Models}

This question arises from simulating the variable geometry of the LM-1600. The principle of variable geometry in gas turbines has been explained in Gas Turbine Theory (Cohen, Rogers and Saravanamuttoo, 1987). From the aerodynamic point of view, variable geometry changes the angle of attack of component blades, so the chances of flow separation and blade stall are reduced. From the thermodynamic point of view, the variable geometry changes the flow area through the component and the overall efficiency. It shifts the component characteristic curve up and down (turbine), or left and right (compressor). Variable geometry improves the off-design performance of the component.

The simulation of a variable geometry gas turbine can be carried out in different ways. To simulate a variable geometry turbine, the most convenient method may be to use the nozzle equation to approximate the generalized turbinc characteristic. 
In the equation, the shape of the curve is tied with the flow area. As the variable vanes open or close, the flow area increases or decreases, and the non-d mass flow at the same pressure ratio increase or decrease too. This application has been shown in some sample calculations, which is very easy to be carried out in a computer model.

The variable geometries of the LM-1600 are all installed in the compressors. Because of the multi-speed characteristic, it brings some difficulties to its simulation. In some traditional methods, compressor characteristic maps for certain geometry positions are stored in the computer. The effect of variable geometry position chang. ing can be simulated by linear or nonlinear interpolation of the maps. Usually, the speed lines of the maps are stored by detailed data files or different polynomials. But the problem is that the data file or the polynomial does not have any physical meaning, and it is also very hard to find the relationship between the position and the polynomial coefficients. So it is hard to use this method in LM16.

A new approach by Muir, Saravanamuttoo and Marsial (1988) has been developed. The method is based on Stage-Stacking analysis, and has been successfully used for the variable geometry compressor of the LM2500-30. The problem to use this method in LM16 is that it needs very detailed information about compressur geometry, the angles of the variable vanes have to be assumed and the stage stacking procedure is complex for a portable PC model.

A nozzle analogy for compressors had been introduced by Csanady (1964). It was thought possible to use a modified nozzle and shaft model to simulate a compressir characteristic. If each speed line of a compressor can be simulated by this model, it wiil become the compressor's mathematic model. Then the simulation of variable geometry compressor would not be difficult. This thought meets with reality on the choking part of speed line, and needs to be modified by aerodynamic considerations near the surge point. If this approach succeeds, it will simplify mary currently used gas turbine rrodels.

\subsection{Conclusions}

As requireci by the user, simulation of the LM-1600 has been carried on a PC model. At the end of this study, the following conclusions could be made: 
1. The mathematical model used is correct. The accuracy of the design point calculation is acceptable.

2. Hot End Method developed for component matching calculation is an efficient computational method.

3. The simulation of the LPC and the HPC of the LM-1600 using the generalized compressor model is successful.

4. The modelling results match with those of available field measurements.

5. The requirem-its on model configuration and output layout have been met.

6. There is still significant work required to develop a simple and effective method to simulate variable geometry in the compressor. 


\section{REFERENCES}

Agrawal, R.K., Maclsaac, B.D. and Saravanamuttoo, H.I.H., 1979, An Analysis Procedure for the Validation of On-Site Performance Measurenents of Gas Turbine, Journal of Engineering for Power, Vol. 101, pp. $405-414$.

Balsa, T.F. and Mellor, G L., 1974, The Simulation of Axial Compressor Performance Using An Annulus Wall Boundary Layer Theory, ASME PaFer No. 74-GT-56.

Barnes, J.F. and Fray, D.E., 1965, An Experimental High-temperature: Turbine, H.M.S.O., A.R.C., R \& M.3405.

Chappell, M.S. and Cockshutt, E.P., 1974, Gas Turbine Cycle Calculations: Thermodynamic Data Tables for Air and Combustion Products for Threc Systems of Units, NRC Aeronautical Report LR-579.

Cockshutt, E.P., 1968, Gas Turbine Cycle Calculatior: Differential Method in The Analysis of Equilibrium Operation, C.A.S.I. Transactions, Vol. 1, No.1; March 1968; pp. $21-38$

Cohen, H., Rogers, G.F.C. and Saravanamuttoo, H.I.H., 1987, Gas Turbine Theory, 3rd edition, Longman Scientific \& Technical.

Csanady, G.T., 1964., Turbomachines, McGraw-Hill Book Company

Farmer, R., 1988, GT-660 Turbine Powered by an LM-1600 Gas Gen-

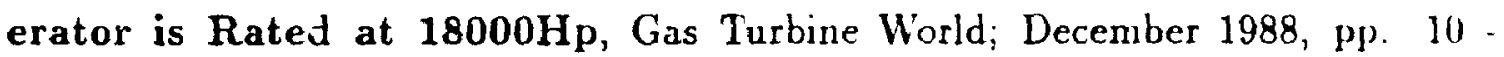
21.

Fawke, A.J. and Saravanamutioo, H.I.H., 1971, Digital Computer Methud for Prediction of Gas Turbine Dynamic Response, SAE Paper No. 7105511

G.E. Company, Gas Turbine Division, 1984, LM-16110 Gas Turbine System, GEZ-7428, 9-84 5M.

G.E. Company, Marine \& Industrial Engines and Service Division, 1985, Design, Performance and Operational Features of the LM-1600 Gas Generator, Brochure.

Hawthorne, W.R., 1956, The Thermodynamics of Cooled Turbines, Part I and II. Trans. Am. Soc Mech. Eng. 78; November 1956; pp. 1765 - 1786.

Hodge, J., 1955, Cycles and Performance Estimation, Butterworth Scientific Publication. 
Matthee, F.A.H and Saravanamuttoo, H.I.H., 1982, Development of A Low Cost Performance Monitoring System for Use on Board Naval Vessels, ASME Paper No. 82-GT-297.

Mattingly, J.D., Heiser, W.H. and Daley, D.H., 1987, Aircraft Engine Design, AIAA Education Series, American Institute of Aeronautic and Astronautics Inc.

Muir, D.E., Sa $a_{i}$ vanamuttoo, H.I.H. and Marshal, Cmdr.D.J., 1988, Health Monitoring of Variable Geometry Gas Turbine for the Canadian Navy, ASME Paper No. 88-GT-77.

Oates, G.C., 1984, Aerothermodynamics of Gas Turbine and Rocket Propulsion, AIAA Education Series, American institute of Aeronautics and Astronautics Inc..

Rohsenow, W.M., Hartnett, J.P. and Ganic, E.N., 1985, Handbook of Heat Transfer Fundamentals, 2nd Edition, McGraw-Hill Book Company.

Saravanamuttoo, H.I.H., and Maclsaac, B.D., 1983, Thermodynamic Models for Pipeline Gas Turbine Diagnostics, Trans. ASME, Journal of Engineering for Power, Vol.105, pp. 875 - 884.

Saravanamuttoo, H.I.H., Sjolander, S.A., Kirkhope, J. and Maclsaac, B.D., 1989, Gas Turbine Performance and Design: Supplementary Courses Notes, Carleton University.

Saravanamuttoo, H.I.H., 1979, A Low Cost, On-Site Performance Monitoring System, ASME Paper No. 79-GT-21.

Saravanamuttoo, H.I.H., 1974, Gas Path Analysis for Pipeline Gas Turbines, Gas Turbine Operation and Maintenance Symposium, National Research Council of Canada.

Saravanamuttoo, H.1.H. and Fawke, A.J., 1970, Simulation of Gas Turbine Dynamic Performance, ASME Paper No. 7C-GT-23.

Sa:vyer, J.W., 1972, Gas Turbine Engineering Handbook, 2nd Ediiion, Gas Turbine Publication Inc.

Scott, J N., 1979, Axial Compressor Monitoring by Measuring Air Intake Depression, Third Symposium on Gas Turbine Operation and Maintenance, National Research Council of Canada.

Scott, J.N., 1977, Improving Turbo Compressor Efficiency via Performance Analysis Techniques, ASME Paper No. 77-Gt-53. 
Urban, L.A., 1973, Gas Path Analysis Applied to Turbine Engine Condition Monitoring, Journal of Aircraft, Vol. 10, No. 7; July 1973; pp. 400. 406.

Williams, L.J., 1981, The Use of Mathematical Modeling in the Analysis of Gas Turbine Compressor Unit Test Data, ASME Paper No. 81-GT-217.

Wittenberg, H., 1976, Prediction of Off-design Performances or Turbojet and Turboían Eı gines Based on Gasdynamic Relationships, Delft University of Technology. 


\section{APPENDIX, COMPUTER PROGRAM}

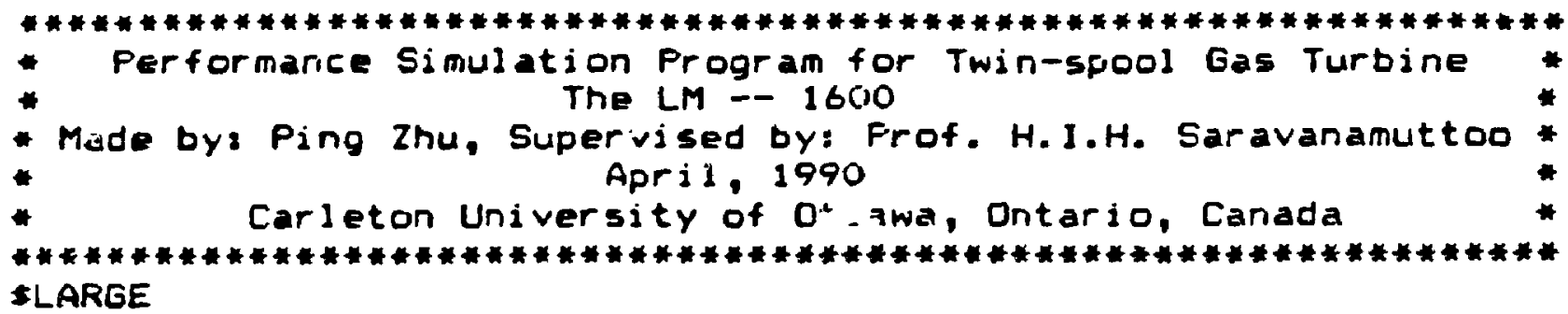

C

MASTER F'ROGFAM FOR LM- 1600

IMPLICIT REAL*B $(A-H, O-Z)$

REAL $* 8$ LET

DIMENSION $A H(6), A C V(6), X M(3), D F 67(3)$

DIMENSION $6(11)$, TFH (40), SPL (6), SFH( 3$)$, SUGEL (6), SUGEH (6)

DIMENSION SFL $1(6)$, SPL2 $(6)$, SFL $3(6), S F L 4(6), S P L E(6)$

DIMENSION SPHI (3), SFHZ (3), SPHJ (3), SFH4 (3), SFHS (3)

DIMENSION FL $(6,2,40), N L(6), P H(6,2,40), N H(6), F R(3)$

DIMENSION AFTL $(10), A F T H(10)$

CHARACTEF $X X$

$M=5$

$M 1=6$

$M 2=7$

$C F A=0.24 D 0$

$C F G=0.275 D 0$

$F \cdot A=1.4 D O$

$F \cdot G=1.33 \Xi D 0$

$E C O M B=0.9900$

$E M E C H=0.9900^{\circ}$

DATA SPL/ $-3.891549610073972, .267072985544958$,

A -.006369223356425, .0000077179026418,

A $-.000000464048699,0000000011053921$

DATA SFL 1/-3.781801200522382,. 25.9980881893007,

A $-.006141926791113, .000074096408811$.

A -. .000000443767222,.0000000010534721

DATA SFL2/-2.885572.919927102,.207094054050981,

A $-.004968200701090, .000061046139850$,

A $-.000000372228769, .0000000008985621$

DATA SPL3/1.058989052629340,-.033963117812704,

A . $000832140701985,-.000007582571043$,

A . $000000027267477,-.000000000017171 /$

DATA SFL4/-4.222536645871570,. 287328689226967,

A - .0068555076554496, .0000082906667696,

A - .000000497274485,.000000001181321/

DATA SFL5/-5.128105823554306,.34014739507001906, A -.008084143807244,.0000969000144781, 
A - . .000000575856603, .000000001355725/

DATA SPH/.893854941050043,-.014061611461038, A .000504748693370/

DATA SPH1/.815030096618174,-.008748411226322, A $.000415538874890 /$

DATA SFH2/.899053649577208,-.014343876670815, A . $000508536705338 /$

DATA SPH3/2.524333564603018,-.124397556791461, A . $002371160308527 /$

DATA SFH4/.845349996778839,-.010988877946578, A .000455389325382/

DATA SFH5/.708662446685995, ,.001680869456876, A .0002979594662821

DATA AH/1.69237766945B20,0.23623B27478711, A $-2.0713577489054 E-005,5.8856351343586 E-008$, A - $3.2313519486(122 E-011,6.0897435245801 E-015 /$

DATA ACV/10413.090210709,-0.30773660204648, A $-2.9583914905551 E-0014,7.156175898003 E-001$, A $-1.0489504005626 E-011,-1.2944573131531 E-021 /$

DATA SUGEL/15.043366562831820,-0.890929589419815. A . $022204020939571,-0.000260412612960$, A $0.000001514340552,-0.000000003483585 /$

DATA SUGEH/21.785469854555010,-4.234613480030412, A . $3389601442657148,-0.012762724566341$, A $6.000238320331315,-0.000001760675300 /$

WRITE $(*, '(A) \cdot)^{\prime}$ TO SEE DESIGN FOINT CALCULATION $(Y / N) ?^{\circ}$ FEAD (*, (A)') XX

IF (XX.EQ. ' $N$ ') THEN

$A H P=33.14457645500$

$A L P=94.09101972400$

$A N=147.41306970406 D 0$

$T 2=447.22 D O$

$E L C D=0.8790671020788200$

EHCD $=0.8735531446739900$

$E P T=1 . D O$

$E L T=.87971770624700$

$E H T=.88698705381900$

$S S 1=.000317414032100$

$S 521=.002921201644900$

$S 522=.001451555528100$

GOTO 910

ENDIF

C DESIGN POINT CALCULATION

10 WFITE (*,*) 'POLYTROFIC EFFICIENCY OF LP COMFRESSOR' FEAD (*,*) PEFL

WRITE $(*, *)$ 'FOLYTFIOFIC EFFICIENCY OF HP COMFFESSOF' READ $(*, *)$ PEFH

WFITE $(*, *)$ 'GIVE THE VALUE OF TS.

FEAD $(*, *)$ TS

$T 1=288.00$ 


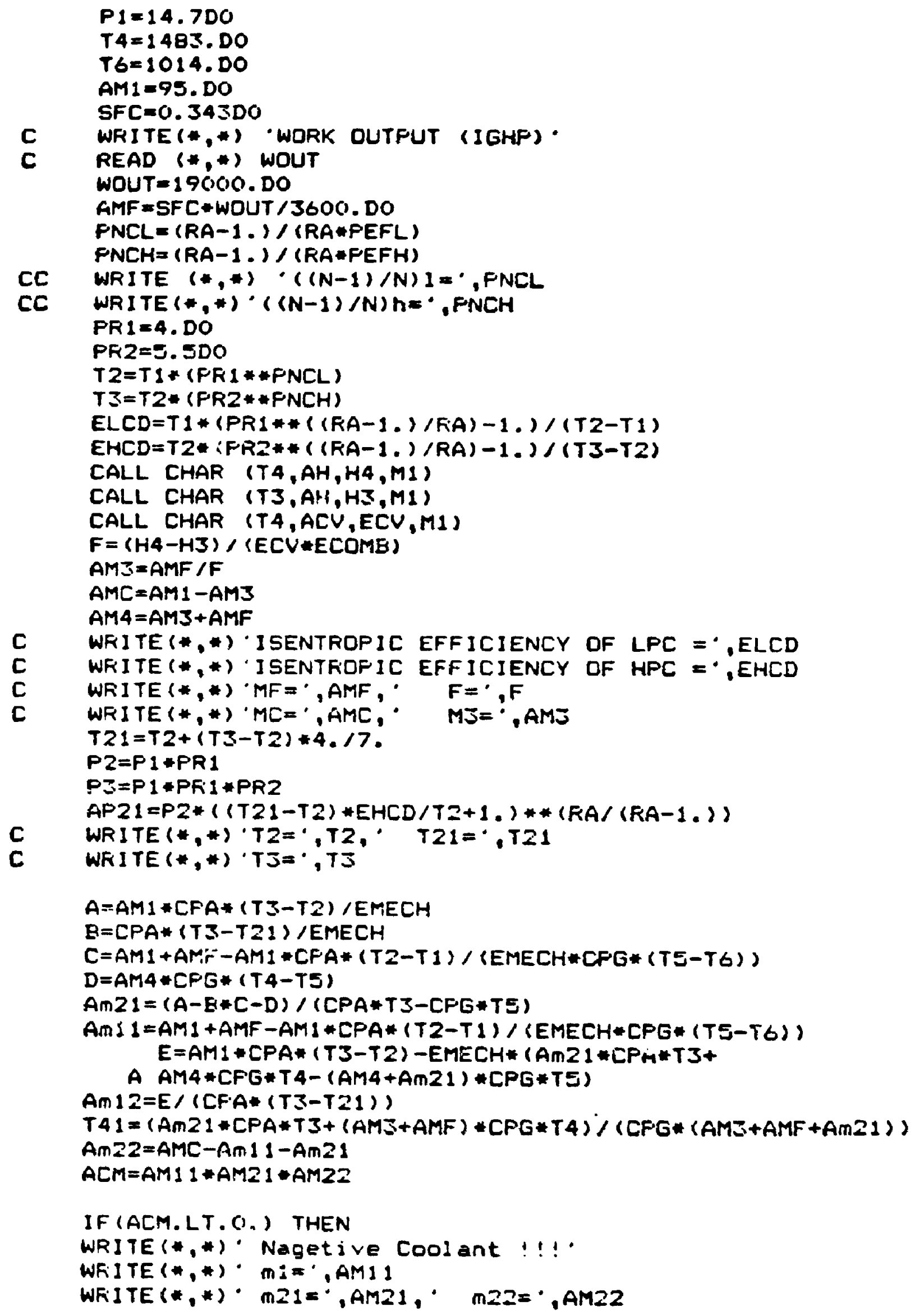




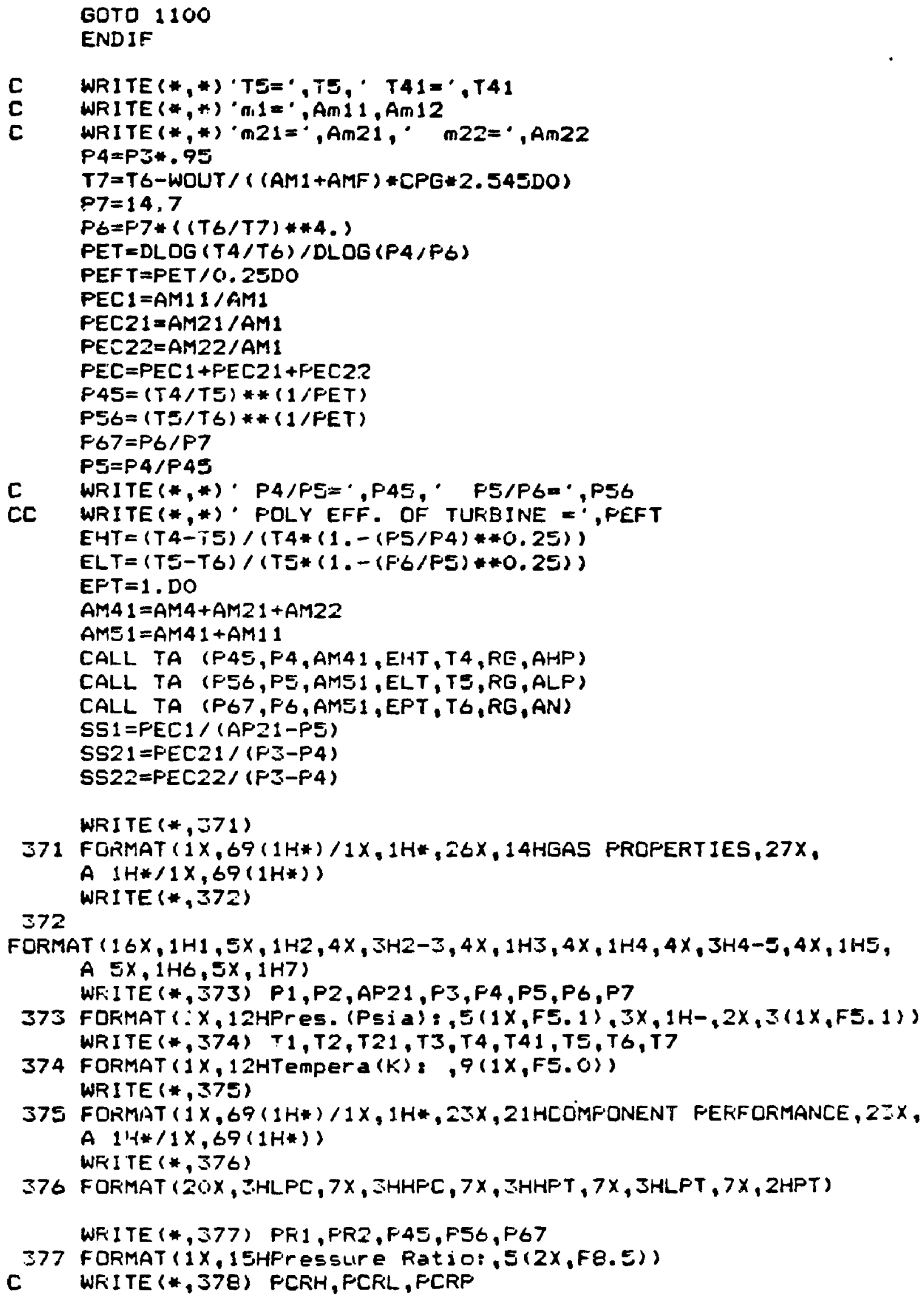


C 378 FORMAT ( $1 X, 17$ HTurbine Choke FR:, 17X,3(2X,F8.4)) WRITE (*,.379) ELCD,EHCD,EHT,ELT,EPT

379 FORMAT ( $1 X, 15 H I$ sentrpoic Eff: $5(3 \times, F 7.5)$ ) WRITE (*,380) AHP, ALP, AN

380 FORMAT ( $1 \times, 29$ HEffective h. Sa (Square inch): , $5 x, 3(2 \times, F B .4)$ ) WRITE (*,378) WOUT,SFC

378 FDRMAT (1X,19HWork Output $(16-H p), 1 X, F 6,0,1 H ;, 7 X$,

A 16HSFC (Ib/IGHp-hr): $\left.1: X, F 6.4,1 H_{\xi}\right)$

WRITE (*,381)

381 FORMAT $(1 X, 69(1 H *) / 1 X, 1 H *, 26 x, 15 H G A 5$ FLOW (ID/S),26X, A $1 H * / 1 \times, 69(1 H *))$

WRITE (*, 382)

382 FORMAT $(5 x, 9 H I n l e t$ air, $2 x, 9 H F u e l$ flow, $2 x, 11$ HCooling air, $2 X$, A 9HHT NOZZ1ะ,2X,8HHT Rotor, $2 X, 6 H L T$ N\&R) WRITE (*, 383) AM1, AMF, AMC, AM21, AM22, AM11

$3 B 3$ FORMAT $17 X, F 5.1,5 X, F 7.4,5 X, F 7.4,2 X, 3(3 X, F 7.4)$ ) WRITE (*, 384) PEC, PEC21, FEC22, FEC 1

I.84 FORMAT ( $1 X, 20$ HPercentage to Inlet: ,8X,F7.5,2X, $3(3 X, F 7.5))$ WRITE (*, 3BE) $5521,5522,551$

385 FORMAT $(1 X, 2$ IHCalculation Constent: $13 X, 3(1 X, E 10.3))$

C WRITE (*,*) ' ISENTROFIC EFFICIENCY OF HPT =",EHT

C WRITE(*,*) * ISENTRDFIC EFFICIENCY OF LPTE",ELT

C WRITE $(*, *)$ ' PERCENTAGE OF COOLANT $={ }^{\prime}$,FEC

C WRITE (*,*), PEC1 $=$,FECI

C WRITE (*,*)'PECZ1 = "PEC21," PEC22 = ,PEC22

C WRITE (*,*)' HFT AREA $={ }^{\circ}$,AHP

C WRITE $(*, *) \cdot$ LFT AREA $=\cdot$,ALF

$\because \quad$ WRITE $(*, *)$ ' FT AREA $=$ ", AN

C WRITE $(*, *)$ ' Sm 1 CONSTANT $=$, SSI

C WRITE (*,*)' Sm21 CONST. $=\cdot, 5 S 21,{ }^{\circ}$ SmZ2 CONST. $=\cdot$,SS22 WRITE $\left(*, \cdot(A)^{\prime}\right)$, ARE YOU FEADY TO DO MAIN CALCULATION (YIN)?

READi*, (A)', $X X$ IF $\left(X X . E O \cdot{ }^{\prime} N\right.$ ') GOTO 10

C LM-1600, COOLING \& MF MODIFICATION, CROSS ITEARATION $910 \quad E F M=0.9900$

$F \cdot 4 \Xi=0.9500$

$S A=0.4 D O / 1.4 D O$

$S G=0.333 D 0 / 1.333 D 0$

$X L O=99 . D O$

$Y L O=3.5500$

$X H O=3 O . D O \#$ SRRT $(T 2 / 447.22 D O)$

$Y H C=4.7 D O$

$D L F 5=12350.20$

$D H F \cdot S=15200 . D O$

OEEN (1,FILE='EFF.DAT')

OF'EN (2,FILE='EFL.DAT')

FEAD $(1, *) \quad(N L(I), I=1,6)$

DO $55 \quad I=1,6$

$\operatorname{FEAD}(1, *) \quad(F L(I, 1, J), J=1, N L(I))$

$\operatorname{READ}(1, *) \quad(F L(I, Z, J), J=1, N L(I))$

55 CONTINUE 


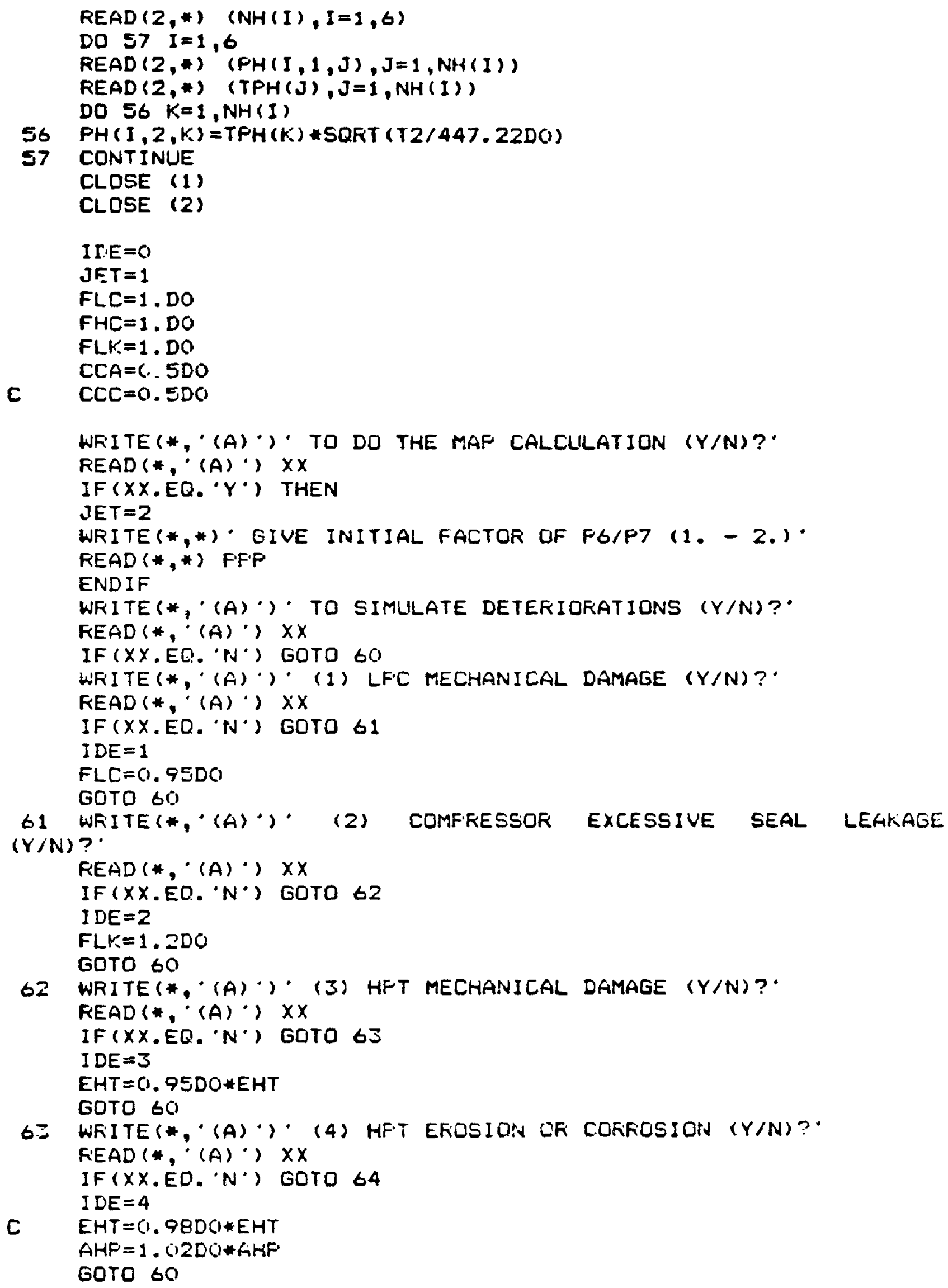




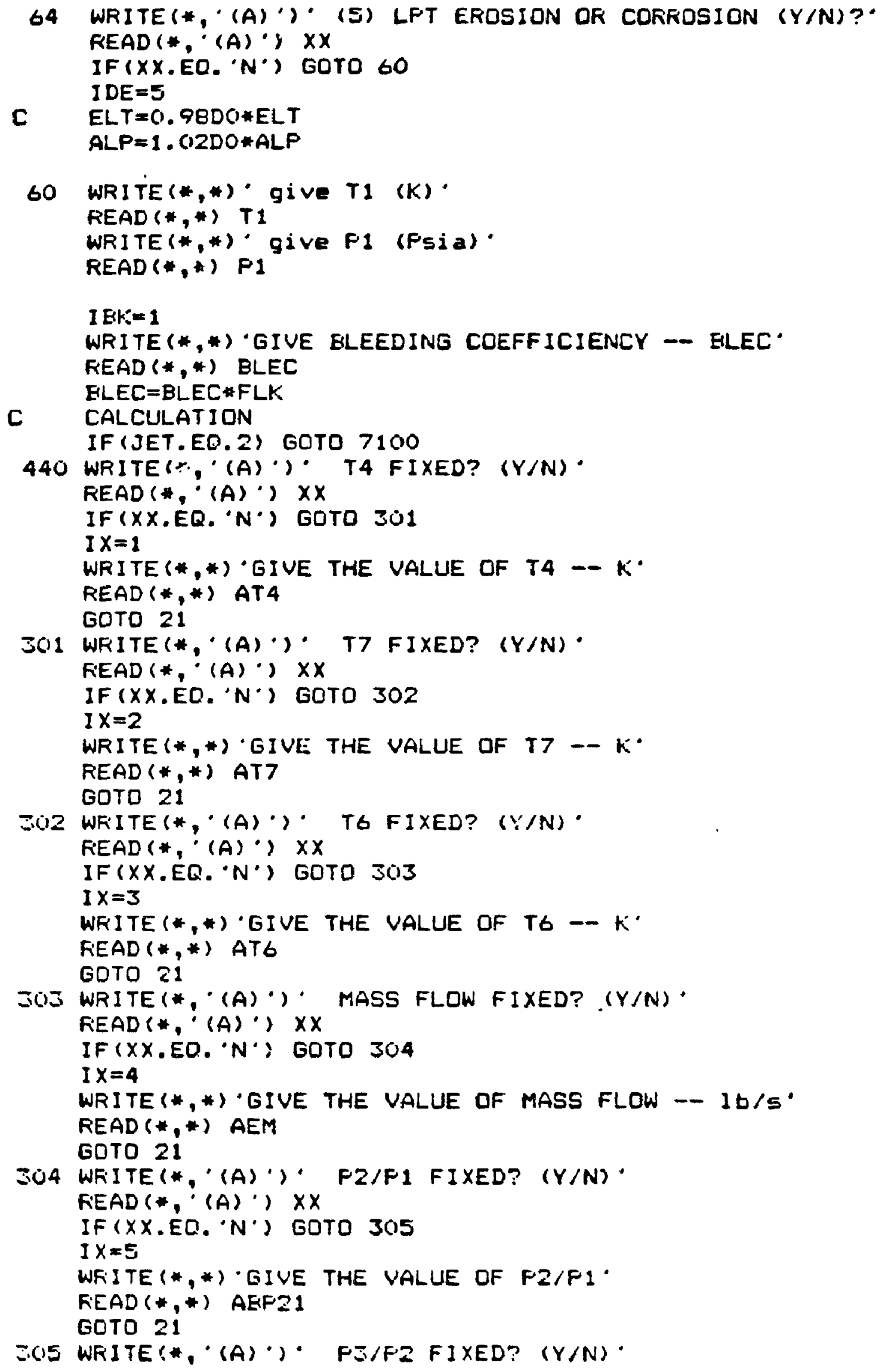


READ (*, '(A)') $X X$

IF $(X X . E Q . ' N$ ') GOTO 306

$I X=6$

WRITE (*,*) 'GIVE THE VALUE OF FI/P2'

FEAD (*, *) AP32

GOTO 21

306 WRITE (*, $(A) \cdot)^{\circ}$ P3/F1 (OPF) FIXED? (Y/N).

READ $(*, \cdot(A) \cdot) \times X$

IF $\left(X X . E Q . ' 14^{\prime}\right)$ GOTO 309

I $X=7$

WRITE (*,*) GIVE THE VALUE DF F3/PI.

READ (*,*) AP31

GOTO 21

C $\operatorname{READ}(*, \cdot(A) \cdot ; \times X$

C IF $(X X, E Q . \cdot N \cdot)$ GOTO $\geq 08$

C I $X=8$

C WRITE(*,*) 'GIVE THE VALUE OF F.4/FE'

C READ (*,*) AFAS

C GOTO 21

C 30 WRITE (*, '(A)')' PS/FG FIXED? (Y/N)'

C READ (*, '(A)') $X X$

C IF $(X X . E Q . ' N \cdot)$ GOTO 309

c I $X=9$

C WRITE(*,*) GIVE THE VALUE OF F.5/F.G.

C FEAD (*,*) AFS6

C GOTO 21

509 WRITE (*, (A) ') WORK OUTFLIT FIXEO? (Y/N) *

READ (*,'(A)') $x X$

IF $(X X . E Q . ' N$ ') GOTO 310

$I X=10$

WRITE $(*, *)$ GIVE THE VALUE OF WOFK OUTPUT -- BHP.

READ (*,*) AWDUT

GOTO 21

$\Xi 10$ WRITE $(*, \cdot(A) ;$ FUEL FLOW FIXED? (Y/N)'

FEAD (*, $(A) \cdot) \times X$

IF $(X X . E Q . ' N \cdot)$ GOTD 311

I $X=11$

WRITE(*,*) 'GIVE THE VALUE OF FUEL FLOW (at DFC Mf=6516) --

Ib/hr

READ (*,*) ABMFH

$A B M F=A E M F H / 3600 . D O$

GDTO 21

311 WRITE $\left(*, \cdot(A)^{\prime}\right)^{\prime}$ LF-SFOOL SFEED FIXED? (Y/N)'

READ (*, '(A)') $X X$

IF $(X X . E Q . ' N$ ') GOTO 21

I $X=12$

WRITE (*,*) 'GIVE THE LF-SPOOL SFEED -- FIFM'

FIEAD $(*, *)$ ARFM

21 WRITE (*,*) GIVE ANY VALLIE OF F.6/F7.

FEAD (*, *) F 67

7100

IF (JET.EO.1) GOTO 7105 


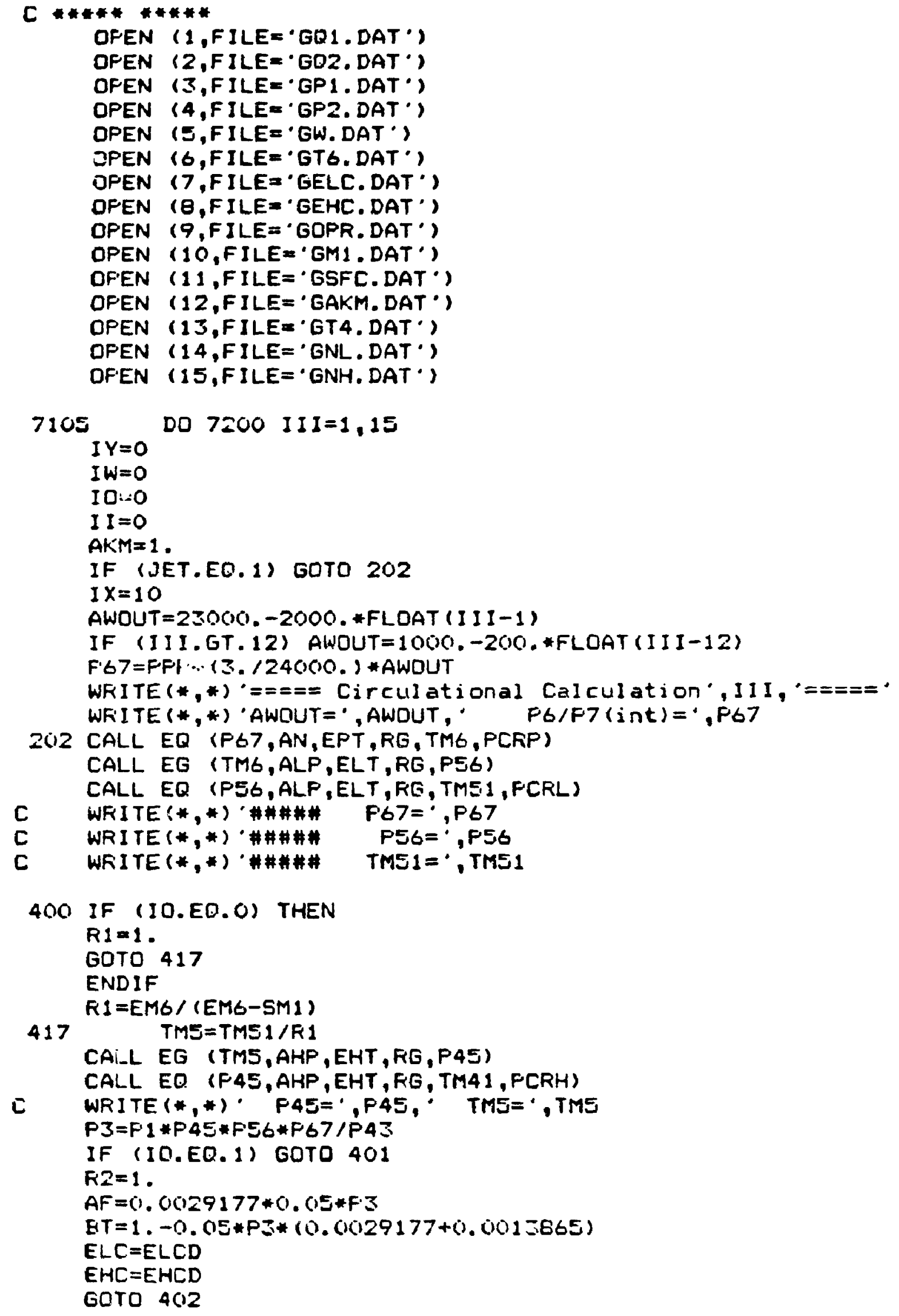


401 RZ=EM1 / (EM6-SMI)

IF (IEK.EQ.2) GOTO 510

IF (TM1.LT.58.) THEN

AK.M $=1 .-(B L E C * \operatorname{SQKT}(T 2 *(1 .-(1 / F 21))) / E M 1)$

GOTO 502

ENDIF

IF (TM1.GT. 108.) THEN

AK:M=1.

GOTO 502

ENDIF

AKM $M=1 .-B L E C *((108 .-T M 1) / 50) * S Q R T.(T 2 *(1 .-(1 / F 21))) / E M 1$

GOTO 502

$510 A F=5 M 21 /(E M 1-3 . * 5 M 1 / 7$.

GDTO 504

502 AF $=S M 21 /(E M 1 * A Y M-3.5 M 1 / 7$.

504 BT $=A F * E M 4 / S M 21$

$402 A=E F M * E L T * E L C * C F G /(C F A * T 1 * R 2)$

$B=1.0-E H T *(1.0-(1.0 / P 45) * 0.25)$

$C=1.0-(1.0 / P 56) * 0.25$

$E=(P 45 * F 56 * F 67 / F 43) * 0.2857$

$A B C=A * E * C$

C WRITE $(*, *), R I=\cdot, R 1, \cdot F Z=\cdot, R 2$

$A K=C F A * T 1 /(E H C * E F M)$

$G=1 .-E H T *(1 .-(1 . / F 45) * * 0.25)$

AK: $1=A F * C F A * T 1 / A K$

$A K: 2=C F G *(E T-(A F+E T) * G) / A K$

$E 1=E-1$.

$E 2=(A B C * E 1 / E L C)-A B C$

$E 3=A B C * A B C / E L C$

$E 4=A B C / E L C$

$E S=1 .+E 1 / E H C$

$E 6=A B C-A E C / E H C$

$E 7=A K 2 * A B C$

$A A=E 7+E 3+A K .1 * E 4 * E 6$

$E B=A K 1 *\{E 4 * E 5+E 6\}+A K 2-E 2$

$C C=A K I * E 5-E 1$

$A G=E E * B E-4.0 * A A * C C$

$T 4=(S Q R T(A G)-E B) /(2.0 * A F)$

CC WRITE $(*, *) \cdot T 4=\cdot, T 4$

T $41=(-\operatorname{SQRT}(G)-\mathrm{BB}) /(2.0 * A A)$

IF (TA1.LT.O.O) GOTO 5

WRITE (*, *) 'T41=',T41

$5 \quad F 21=(1.0+A E C * T 4) * 3.5$

C WFITE (*,*),P21 = , F'21

$T 2=T 1 *(1.0)+(1.0) / E L C) *(F 21 * 0.2857-1.0))$

TM2 $=(P 2 * A K M * T M 41 * F 45 * F \cdot 56 * F \cdot 67 * S O F T(T 2 / T 4) / F 21)$

$T M 1=T M 2 * F 21 * S O R T(T 1 / T 2) / A K M$

$F \cdot 2=F 1 * F 21$ 


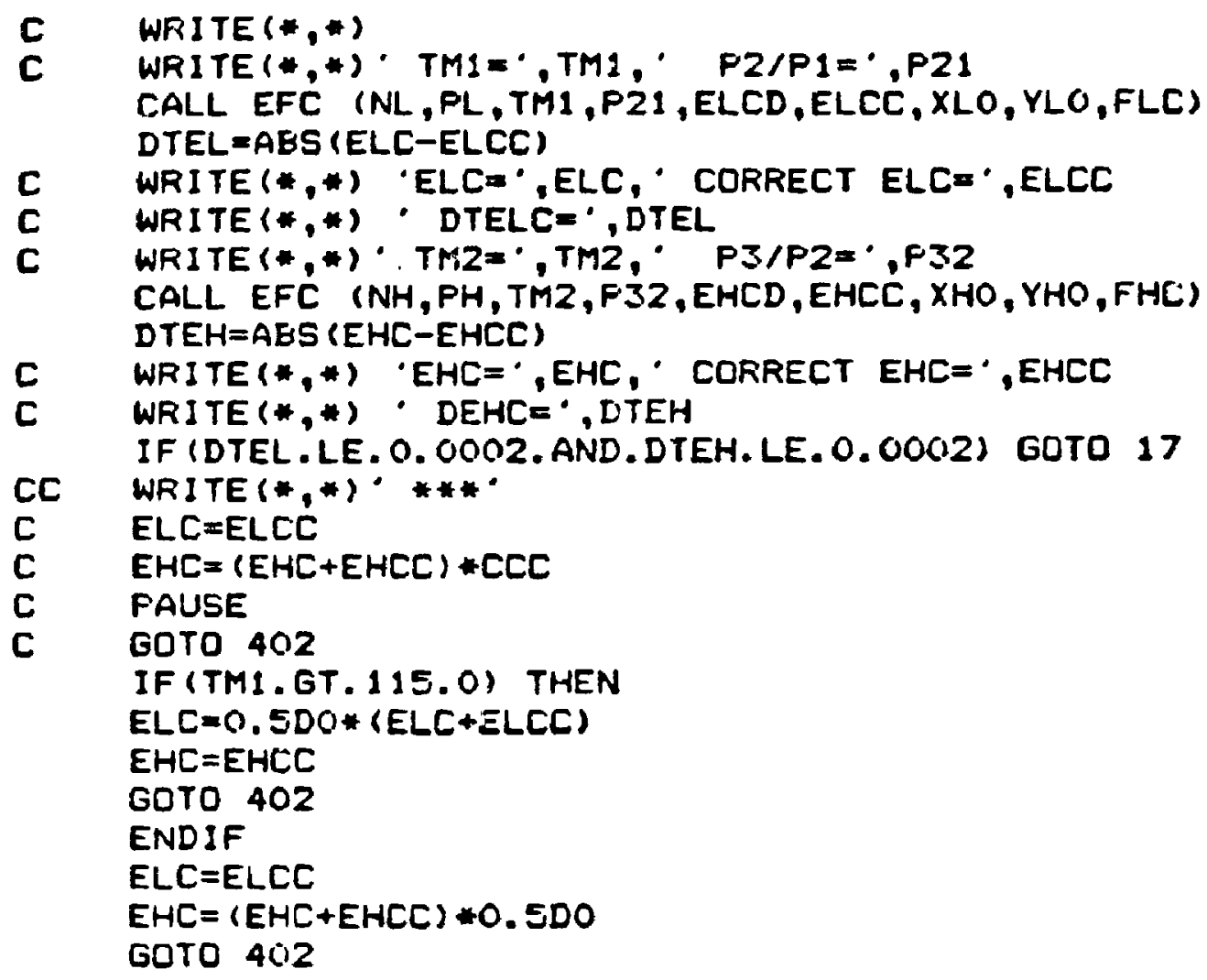


$5 M 2.1=S 521 * D P 34 * E M 1$

$S M 22=5522 * D P 34 * E M 1$

DSM1 $=$ ABS (SM1-SMM1)

DSM2 =AES (SMM21-SM21)

DSM $=A B S$ (SMM22-SM22)

$D S M=D S M 1+D S M 2+D S M 3$

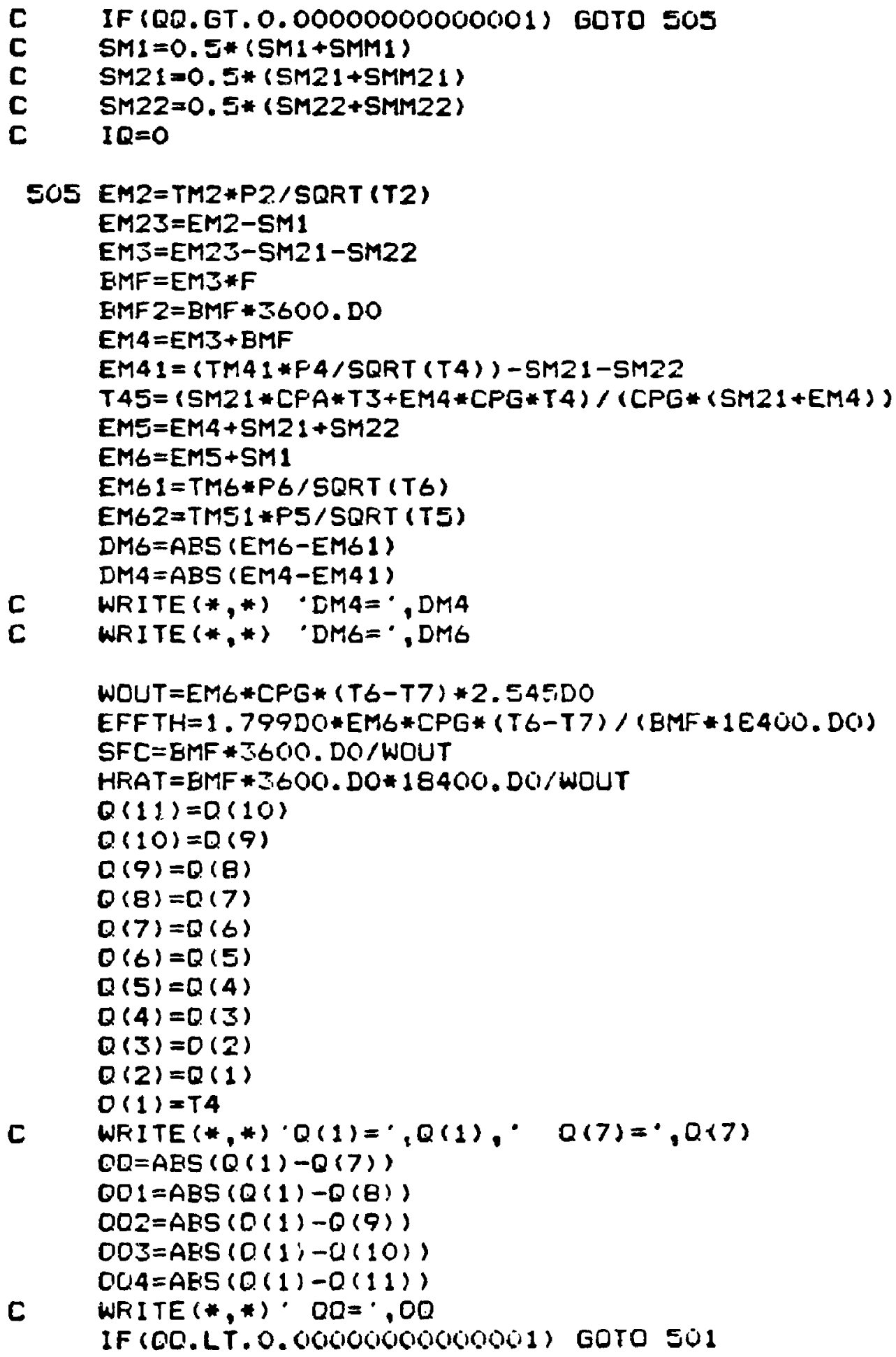




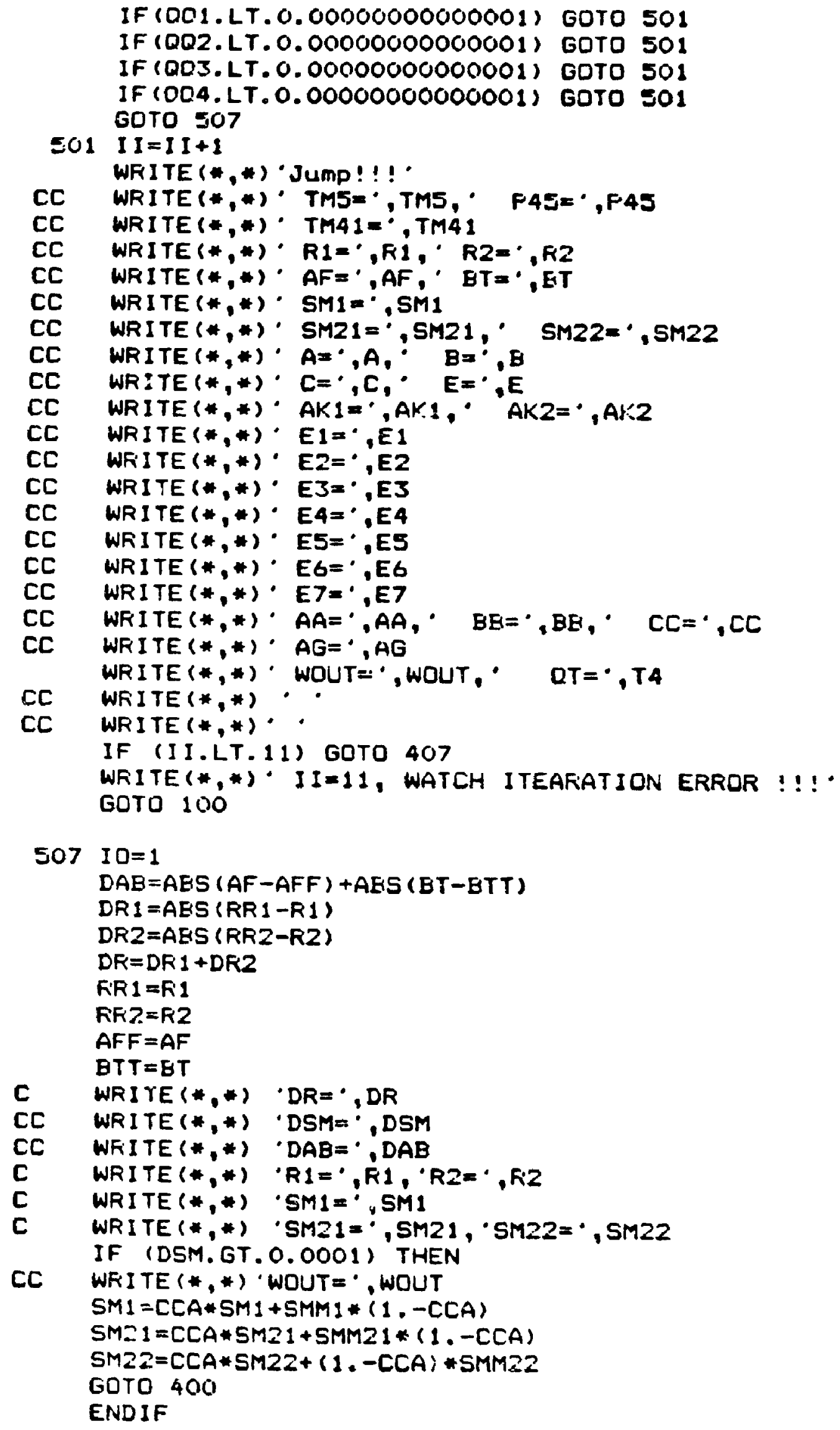




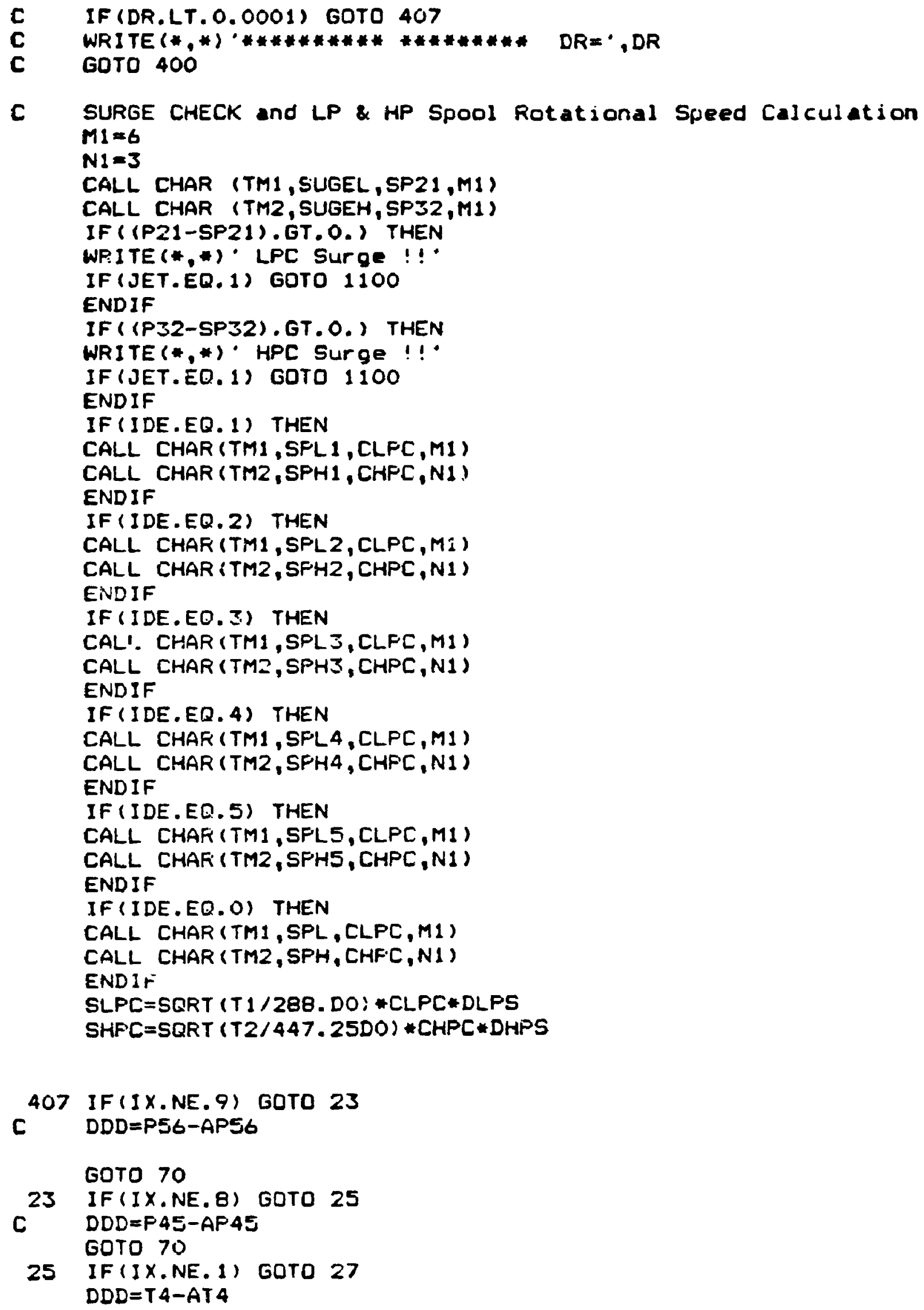




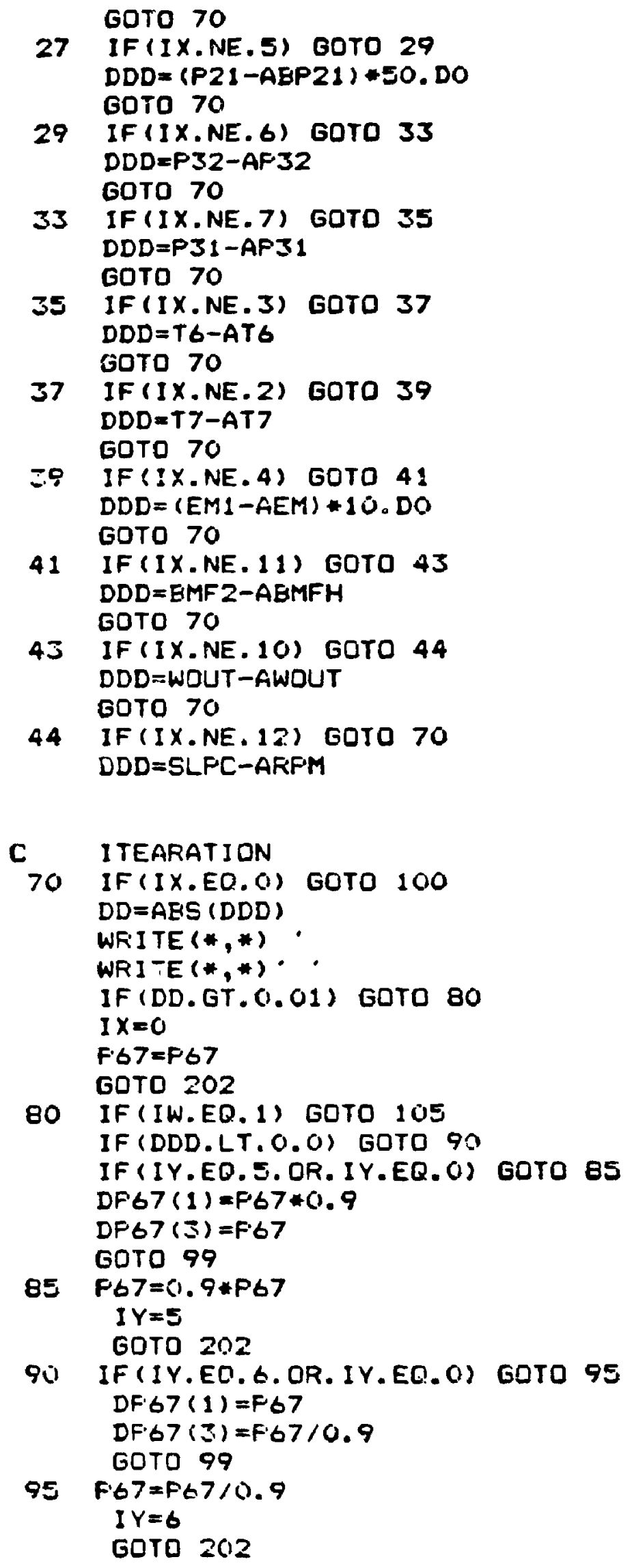

41 IF (IX.NE.11) GOTO 43 DDD $=E M F 2-A B M F H$

44 IF (IX.NE. 12) GOTO 70 $D D D=S L F C-A R F M$

C ITEARATION

70 IF(IX.EO.O) GOTO 100 $D D=A B S(D D D)$

80 IF (IW.EQ.1) GOTO 105 IF (DDD.LT.O.O) GOTO 90

$85 \quad P 67=0.9 * P 67$

IY $Y=5$

GOTO 202

90 IF(IY.EO.6.OR. IY.EQ.O) GOTO 95 DF. $67(1)=F 67$

$95 \quad F \cdot 67=F 67 / 0.9$

$I Y=6$

GOTO 202 


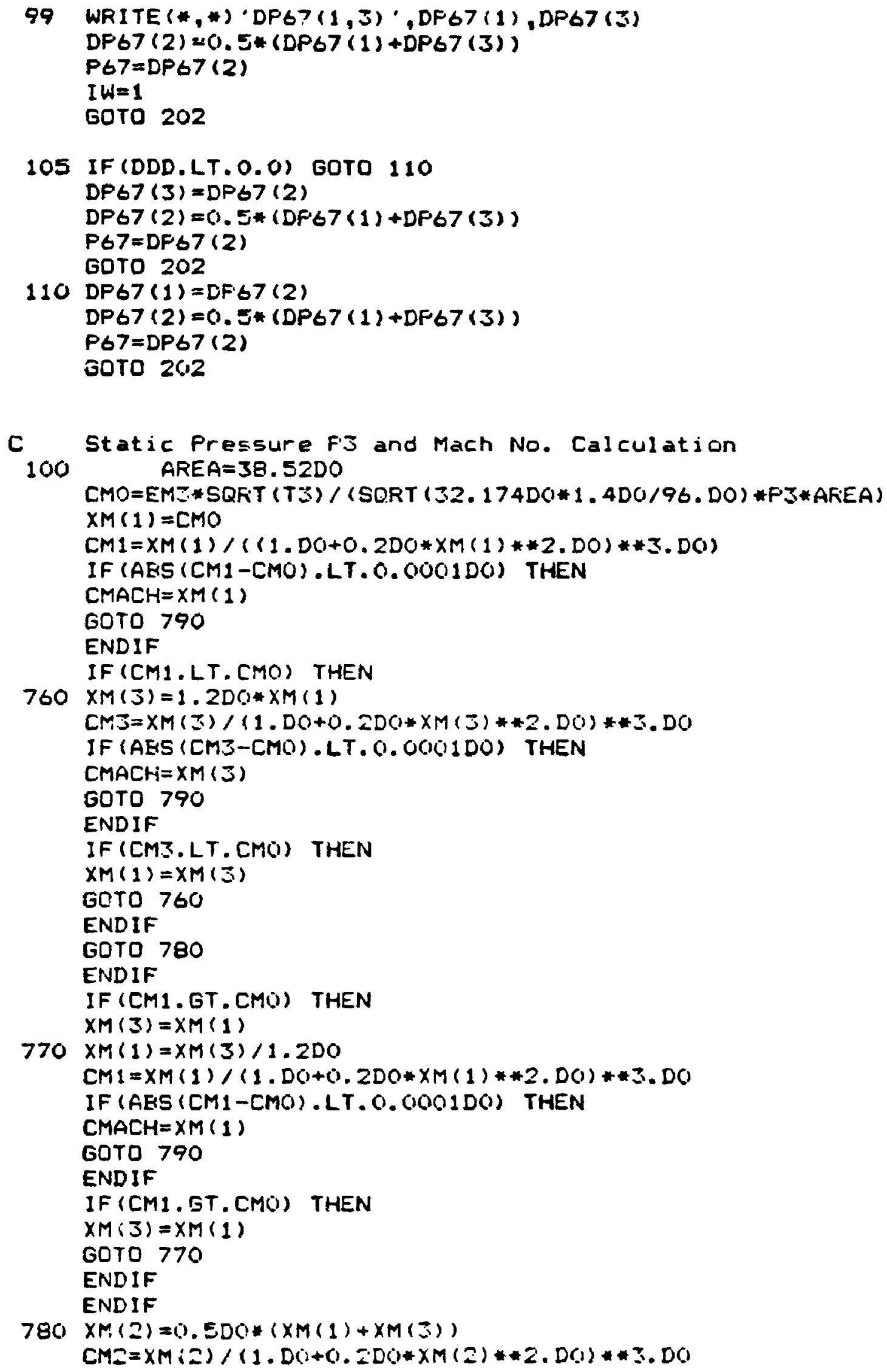




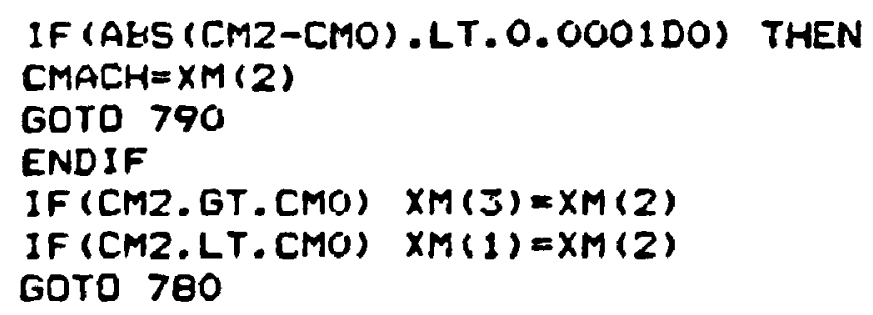

$790 \mathrm{SP3}=\mathrm{PJ} /((1, \mathrm{DO}+.2 \mathrm{DO} * \mathrm{Cr} \cdot \mathrm{ACH} * 2 . \mathrm{DO}) * 3.5 \mathrm{DO})$

$S P 31=5 P 3 / F_{1}$

WLPC $=E M 1 * C F A *(T 2-T 1) * 2.545 D O$

WHPC $=(E M 2 * C F A *(T 21-T 2)+E M 23 * C P A *(T 3-T 21)) * 2.545 D 0$

WHPT $=E F M * C F G *(S M 21+E M 4) *(T \triangle 5-T 5) * 2.545 D 0$

WLF'T $=E F M * C F \cdot$ EME * $(T 5-T 6) * 2.545 \Omega O$

FLLF =WLPC /WLPT

FHF $=$ WHPC $/$ WHPT

RRR=RLF $*$ RHP

$C M Z 1=5 M 21 / E M 1$

$C M 22=5 M 22 / E M 1$

$C M 1=5 M 1 / E M 1$

$B M=E M 1 *(1 . D O-A K M)$

$R E M=1 . D O-A K M$

WOUT $1=0.64801$ DO*WOUT

BMF $1=$ EMF 2*0. 45 3600

C OUTPUT

WRITE (*, 71$)$

71 FORMAT $(1 X, 69(1 H *) / 1 X, 1 H *, 26 x, 14$ HGAS FFOFEFITIES,27X, A $1 H * / 1 X, 69(1 H *))$

WRITE $(*, 72)$

72 FORMAT ( $1 X, 3$ HSt.,2X, 14 HFressure (F'sia),

A $2 X, 14 H T$ mperature $(K), 2 X$,

A 16 HMass Flow (Ib/S), $2 X, 13 H N-D$ Mass Flow)

WRITE (*,73) F1,T1,EM1,TMI

73 FORMAT ( $1 X, 1 H 1,6 X, F 9.4,7 X, F 10.4,7 X, F 8.4,7 X, F 9.4)$

WRITE (*,74) F2,T2,EM2,TMZ

74 FORMAT (1X,1H2,6X,F9.4,7X,F10,4,7X,F8.4,7X,F9.4)

WFITE (*,75) AP21,T21, EM25

75 FQRMAT ( $1 X$, IHI $-3,4 X, F 9.4,7 X, F 10.4,7 X, F B .4)$

WRITE (*,76) $F 3, T 3, E M 3$

76 FORMAT ( $1 X, 1 H 3,6 X, F 9.4,7 X, F 10.4,7 X, F 8.4)$

WFITE (*,77) P4, T4,EM4,TM41

77 FORMAT ( $1 X, 1 H 4,6 x, F 9.4,7 X, F 10.4,7 X, F 8.4,7 X, F 9.4)$

WFITE (*,78) T45

78 FORMAT (IX, 3H4-5, 20X, F 10.4$)$

WRITE (*,79) P5,T5,EMS,TMS 1

79 FORMAT $(1 X, 1 H 5,6 x, F 9.4,7 X, F 10,4,7 X, F 8,4,7 X, F 9.4)$

WFI TE (*,81) FG,T6,EMG,TMG

81 FORMAT (IX,1H6,6X,F9.4,7X,F10.4,7X,F8,4,7X,F9.4)

WFITE (*,82) F7,T7 
FORMAT $11 X, 1 H 7,6 \times, F 9.4,7 \times, F 10.4)$

WRITE $(*, 181)$

181 FORMAT $(1 X, 69(1 H *) / 1 X, 1 H *, 23 x, 21 H C O M P O N E N T$ FERFORMANCE, $23 X$, A $1 H * / 1 X, 69(2 H *))$

WRITE (*,281)

$2 E 1$ FORMAT $(20 X, 3 H L F C, 7 X, 3 H H P C, 7 X, 3 H H P T, 7 X, 3 H L P T, 7 X, 2 H F T)$

WRITE $(*, 182)$ TM1,TM2,TM41,TMS1,TMG

182 FORMAT $(1 X, 15 H N-D$ Mass FIOW, $5(2 X, F B, 4))$

WRITE (*,183) P21,P32,P45, P56,P67

183 FORMAT $(1 X, 15$ HPressure Ratio:, $5(2 X, F 8.5)$ )

WRITE $*, 184$ ) PCRH,FCRL,FCRP

184 FORMAT $(1 X, 17$ HTURbine Choke FRz, 17X, $3(2 X, F 8.4))$

WRITE (*,1B6) ELC,EHC

186 FORMAT $(1 X, 11$ HEFfiCienCY: $4 X, 2(4 X, F 6.4))$

WRITE $(*, 187)$ WLPC, WHFC, WHFT, WLFT, WOUT

187 FORMAT $(1 X, 12$ HWORK (IGHF) $1,3 X, 5(2 X, F 8.1))$

WRITE $(*, 185)$ SLPC, SHPC

185 FORMAT $1 X, 1$ SHLP SpOOI SpeEd: $2 X_{1}$ F6.0,1X,4HFpm; $7 X$,

A 15HHP SpOOI SpeEd, 2X,F6.0,1X,4HRPM;)

IF (JET.EQ. 1) PAUSE

WR!TE (*,188)

188 FOFMAT $(1 X, 69(1 H *) / 1 X, 1 H *, 26 x, 15 H C O M F F E S S O R$ DATA, 26X, 1H*/

A $1 \times, 69(1 H+1)$

IJRITE $(*, 189)$

189 FORMAT (9X, $18 \mathrm{HP3}$ (Stagnation)/F01,4X,14HP3(Static)/PO1,4X.

A 11 HMach No. (3))

WRITE (*,190) P31, SF $31, \mathrm{CMACH}$

190 FOFMAT $(13 X, F B .5,15 X, F 9.5,10 X, F 5.4)$

WFITE $(*, *)$

WR:TE $(*, 191)$

191 FORMAT $(1 x, 69(1 H *) / 1 x, 14 *, 21 x, 24 H C O C L I N G$ and BLOWING

A FLOW, 22X,

A $1 H * / 1 X, 69(1 H *))$

WRITE $(*, 192)$

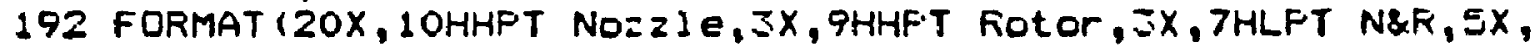

A $11 \mathrm{HBI}$ OWIng Off)

WFITE (*,193) SM21, SMZ2, SM1, EM

193 FORMAT (1X,17HFIOW Rate (It/5): $x, F 7,4,5 X, F 7,4,4 X, F 7,4,7 X$,

A FB. 4)

WRITE (*,194) CM21, CM22,CM1, REM

194 FORMAT $11 X, 20 H$ Ratio to Inlet

A Flow: ,2X,F7.5,5X,F7.5,4X,F7.5,6x,

A F9.5)

WRITE $(*, *)$

WRITE $(*, 195)$

195 FORMAT $(1 x, 69(5 H *) / 1 x, 1 H *, 20 x, 27$ HOUERALL ENGINE FERFCFMAINCE,

A $20 x, 1 H * / 1 X, 69(1 H *))$

WFITE (*,196) WOUT, WOUT 1

196 FORMAT ( $1 X, 1$ 2HWOrk: Output: $5 x, F 10,4,1 X, 5 H I G H P ; 15 X, F 10,4,1 X$, A (HSH-KiW:)

WFI ITE (* 197) EMF, EMF 2, EMF 1

197 FORMAT ( $1 x, 15 H F u e 1$ Flow Fate: $3 x, F 9,6,1 x, 5 H I 0 / 5,2 x, F 7.0,1 x$, 
A $6 H I B / h r ; 2 X, F 7 .(1,1 X, 6 H K g / h r ;)$ WRITE (*,198) F,EFFTH

198 FORMAT (1X,15HFUE 1 Air Ratio: $3 X, F 9.6,1 X, 1 H ;, 5 X$, A 19HThermal Efficiency:, $5 x, F 7.4,1 X, 1 H_{i}$ ) WFITE (*,199) SFC,HFAT

199 FOFMAT 1 XX,4HSFC: , 3X,FB.5,1X,12HIb/IGHP-hr; , 5X, 1 OHHEat A Rate:,

A $3 X, F 11.4,1 X, 13 H B T U / I G H p-h r$;)

IF (JEY.EO.1) GOTO 1100

WRITE $(1, *)$ TMI

WRITE $(2, *)$ TMZ

WRITE $(3, *)$ P21

WRITE $(4, *)$ P32

WRITE $(5, *)$ WOUT

WRITE $(6, *)$ TG

WRITE $(7, *)$ ELC

WRITE $(B, *)$ EHC

WRITE $(9, *)$ SF3L

WRITE $(10, *)$ EMI

WRITE $(11, *)$ SFC

WFITE $(12, *)$ AKM

WRITE $(13, *)$ T4

WRITE $(14, *)$ SLFC

WFITE $(15, *)$ SHFC

7200 CONTINUE

C

CLOSE (1)

CLDSE (2)

CLDSE (3)

CLDSE (4)

CLDSE (5)

CLOSE (6)

CLOSE (7)

CLOSE ( 8 )

CLOSE (9)

CLOSE (10)

CLOSE (11)

CLOSE (12)

CLOSE (13)

CLOSE (14)

CLOSE (15)

1100 CONTINUE

STOP

Eivd

SUERDUTINE TA (F12,F1, AM,EFF, $T 1, F, A R E A)$

IMFLICIT REAL $B(A-H, O-Z)$ 
$P C R=1.0 /((1.0-(R-1) /.((R+1) * E F F)) *.(R /(R-1))$.

IF (P12.LT.PCR) GOTO 10

$A T M=((1 .-(R-1) /.((R+1) * E F F)) *.(R /(R-1)))$.

A (SORT $(R *(R+1) / 2)$.

GOTO 20

$10 \quad P R=1 . / P 12$

$A=S Q R T((2 . * R * E F F /(R-1)) *.(1 .-P R *((R-1) / R))$.

$T M=1 .-E F F *(1 .-F F *((R-1) / R))$

$A T M=P R * A / T M$

20

AREA $=A M * S Q R T(T 1) /\left(.5789 * A T M * F_{1}\right)$

RETURN

END

SLIBROLITINE EQ (F, AFEA, EFF, R, TTM,F'CF)

IMPLICIT FEAL*B $\left(A-H_{3}, D-Z\right)$

C WRITE $(*, *) * *$ EQ ***

$F C R=1.0 /((1 . C-(R-1) /.((R+1) * E F F)) *.(F /(F-1))$.

C WRITE $; *$, ) 'PCR $=\cdot, F C R$

IF (P.LT.FCR) GDTO :O

ATM $=((1 .-(R-1) /.((F+1) * E F F)) *.(F /(R-1)))$.

A (SQRT (F* $*(F+1) / 2)$.

GOTO 20

$10 \quad F F=1 . / P$

$A=S O R T((2 . * F * E F F /(F-1)) *.(1 .-F F *((F-1) / F))$.

$T=1 .-E F F *(1 .-F F *((R-1) / F))$

$A T M=F \cdot R * A / T$

$20 T T M=0.5789 * A F E A * A T M$

C WRITE (*,*)'TM $=$ * TTM

RETURN

END

SUEROUTINE EG (TMM, AR, EF, FR, FRS)

IMPLICIT REAL $\#(A-H, D-Z)$

DIMENSION DF (3)

C WRITE $(*, *) * * * E G * * * *$

FRS $=1$.

IN $N=0$

5 CALL EQ (PRS, AR, EF, FR, TM, FN)

ETM $=T M * P R S * S Q R T(1 .-E F *(1 .-(1 . / F \cdot F) *((R R-1.) / F F)))$

C WFITE $(*, *) \cdot P R S=\cdot$,FRS

$D T M=T M M-E T M$

$D=A E S(D T M)$

C WFITE (*,*) DTM $=\cdot, D T M$

IF (D.LT.O.OON) GOTO $40^{\circ}$

IF (IN.EO. 1) GOTO 20

IF (DTM.LT.O.O) GOTO 10

PFS $=1.4 * F \cdot F$

GOTO 5

10 IN $=1$

$D F \cdot(1)=F F \cdot S / 1.4$

$D P(\Xi)=F F S$ 


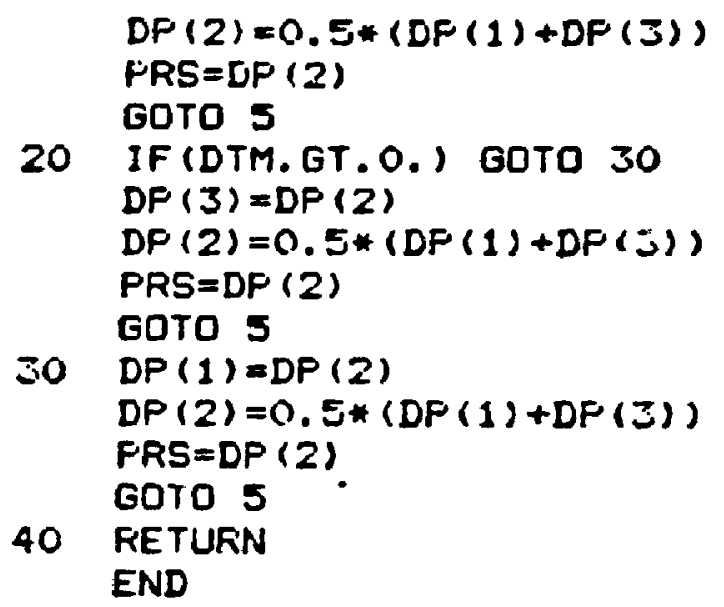




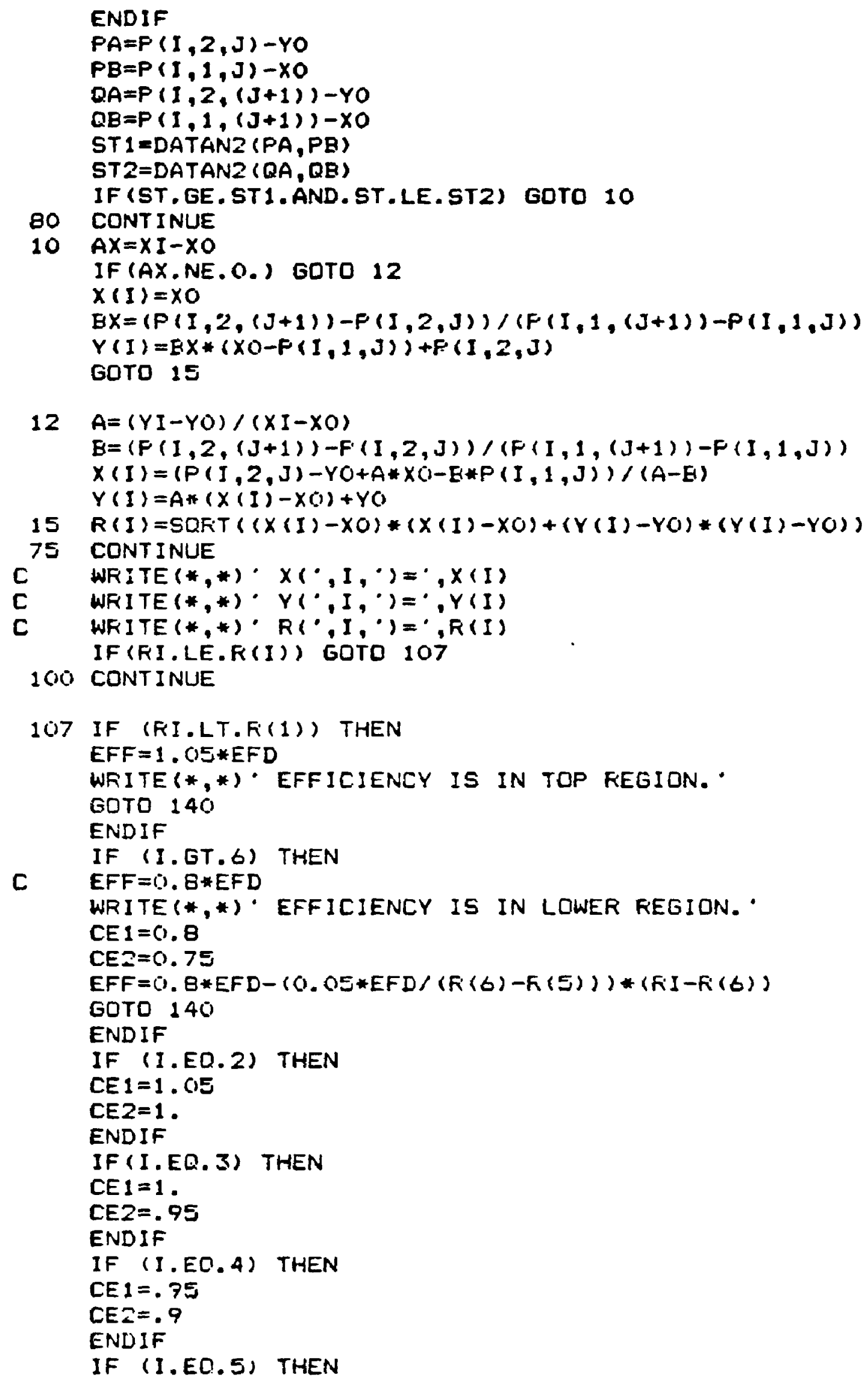




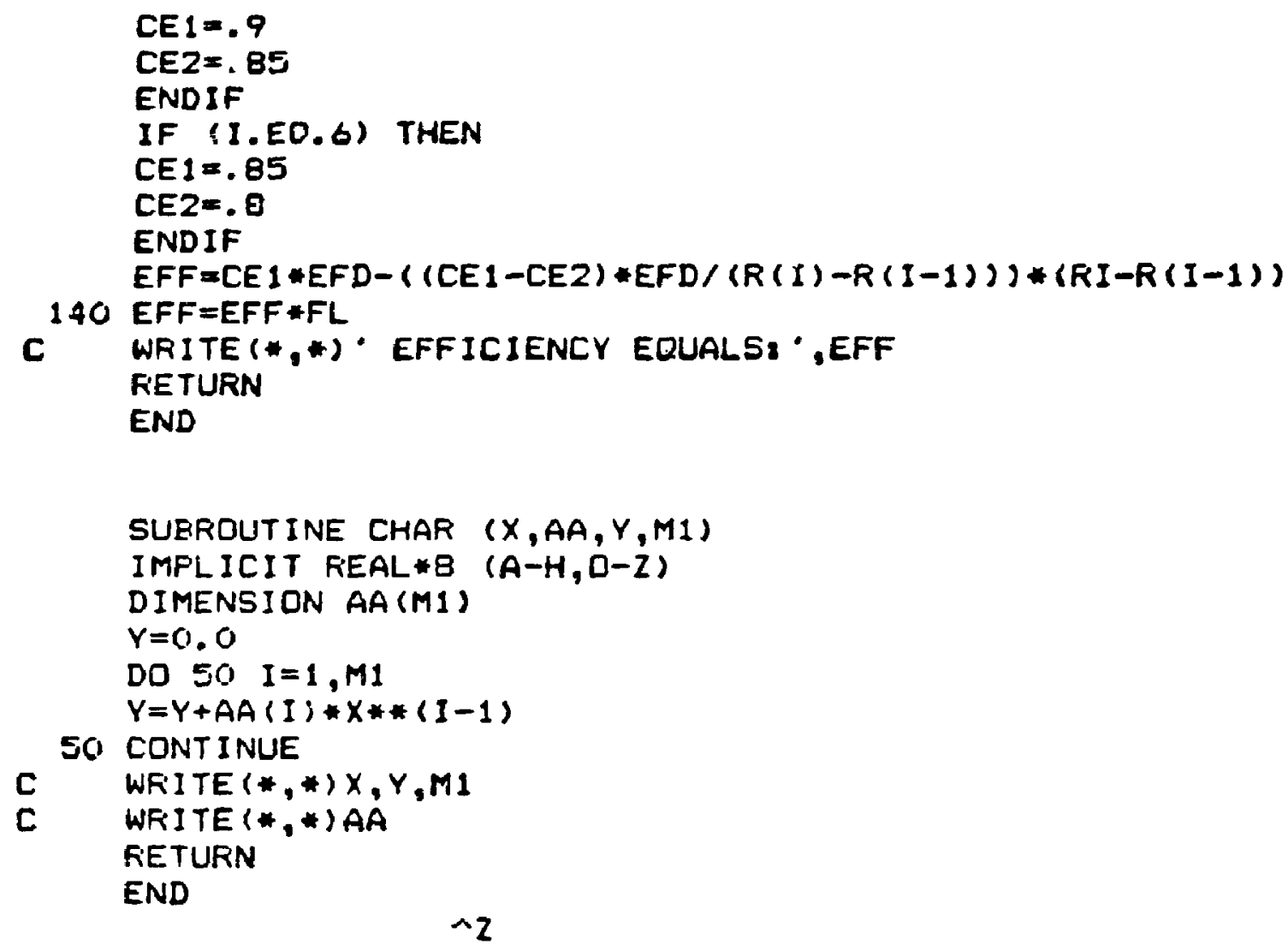




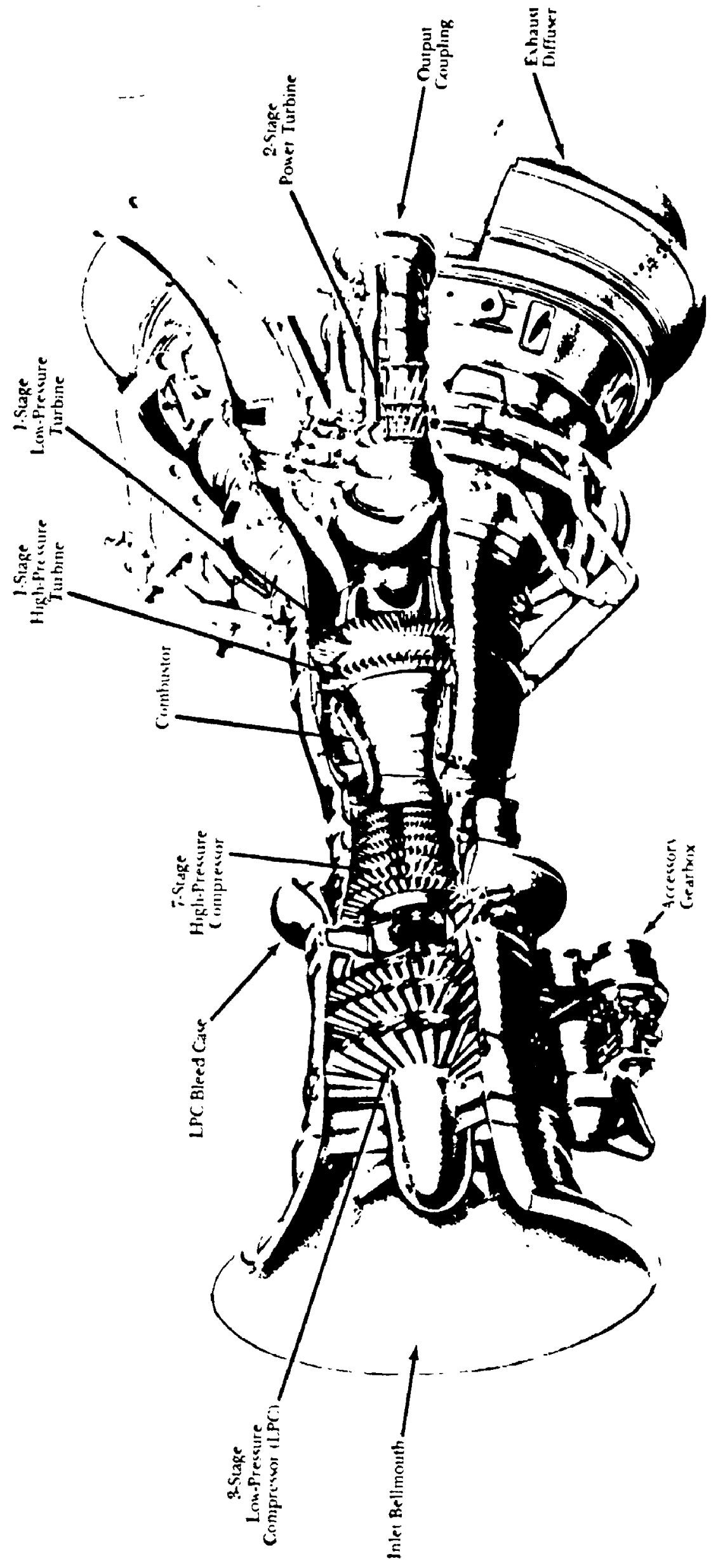

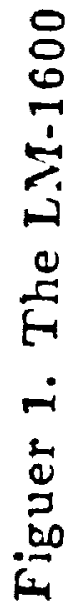




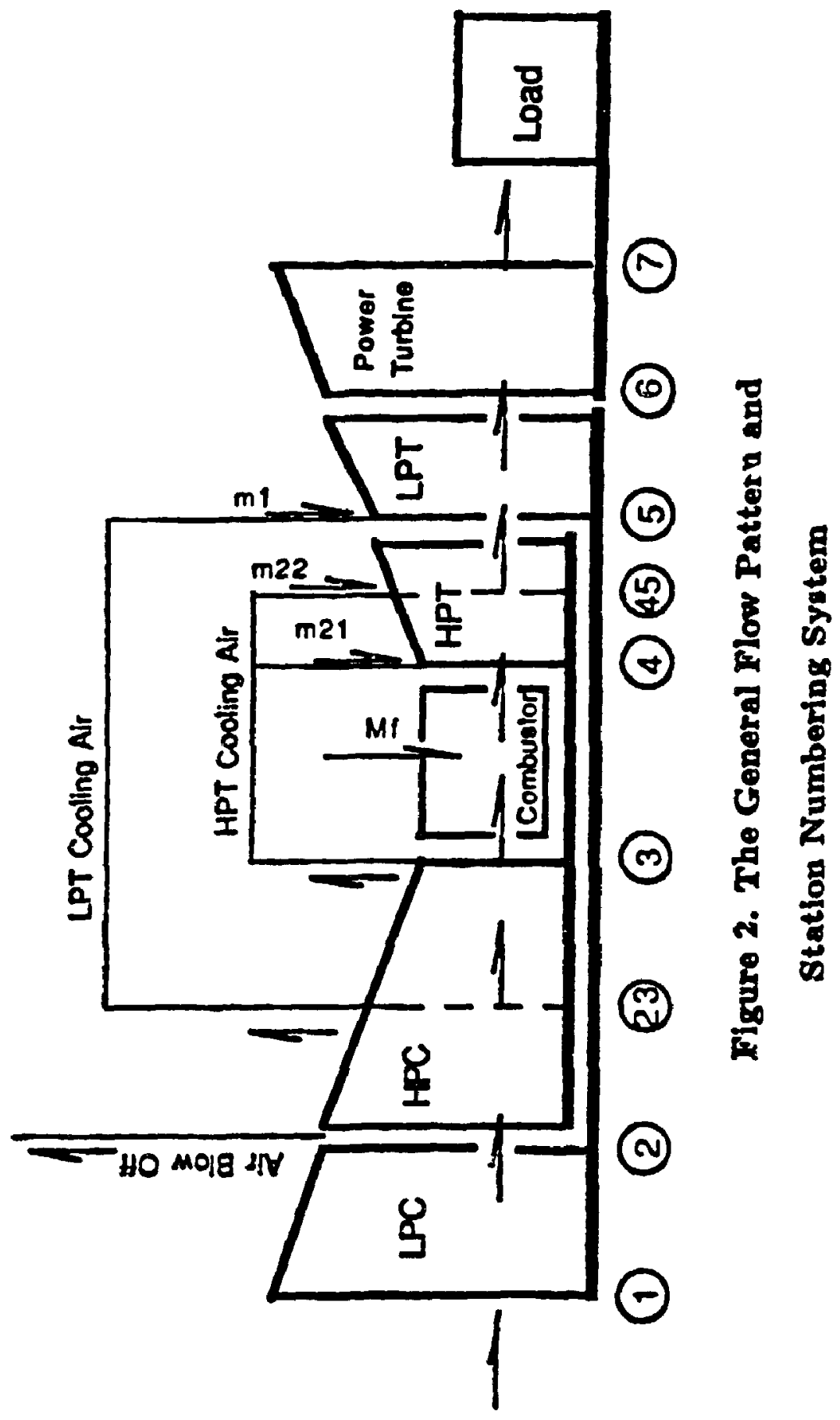




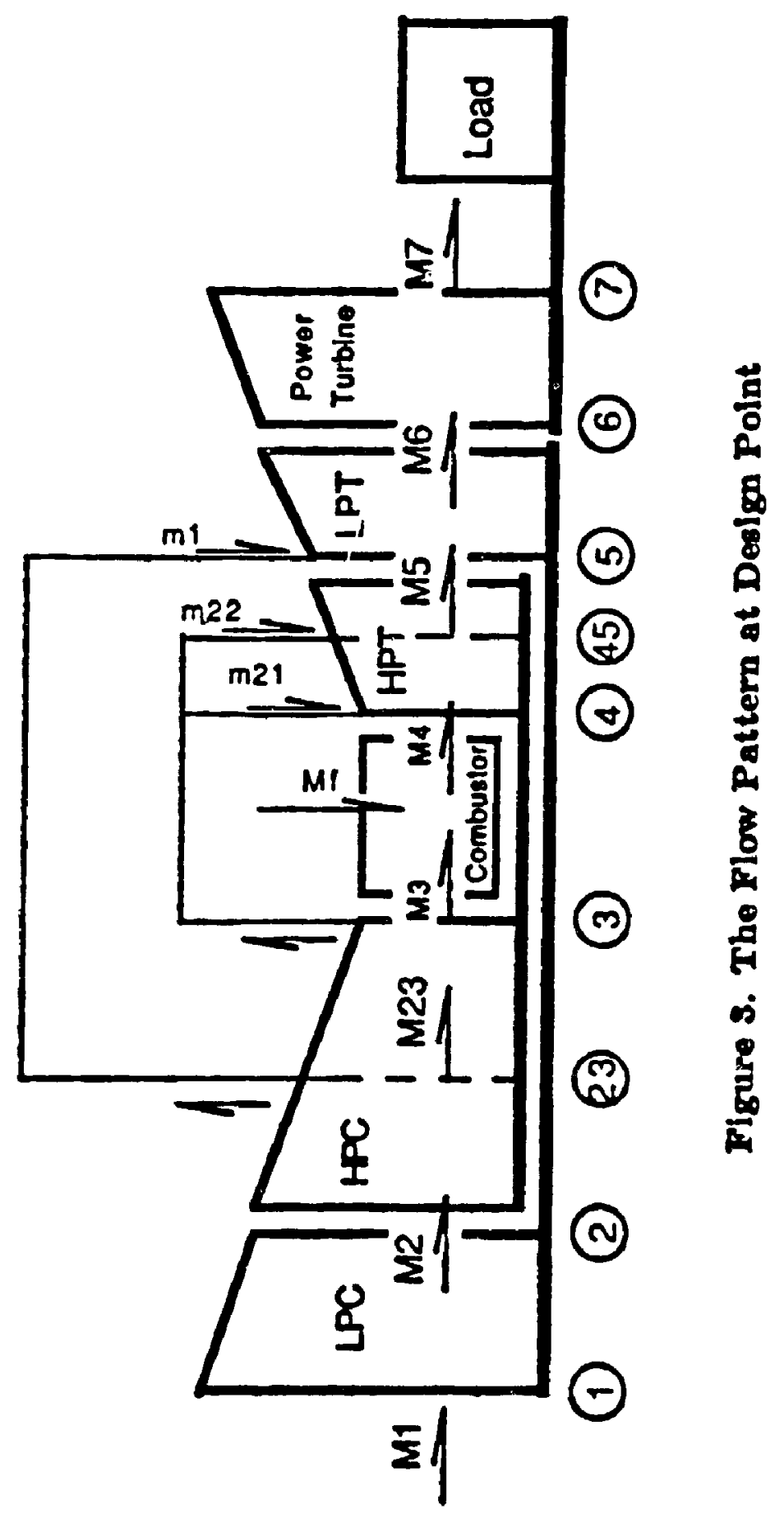




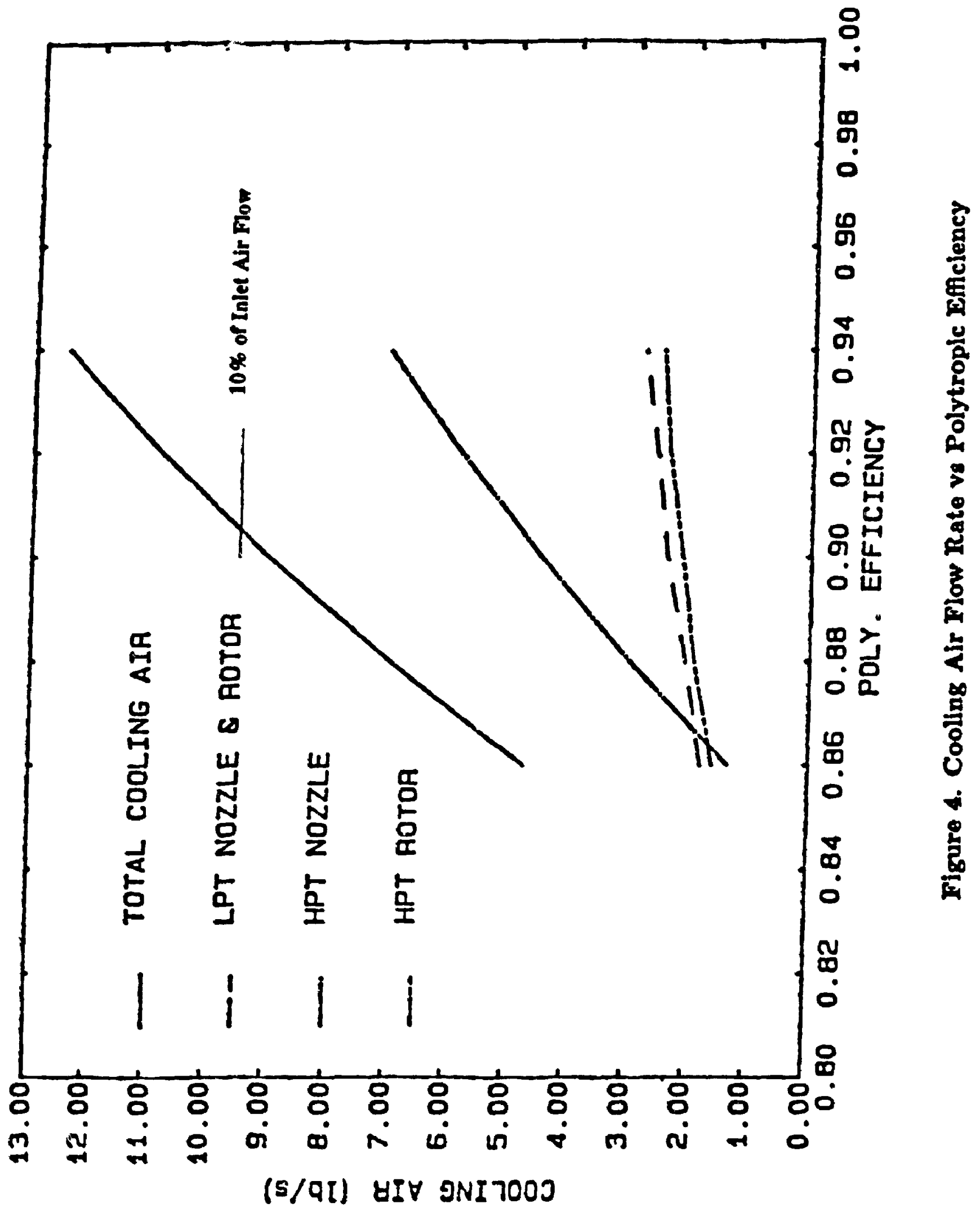




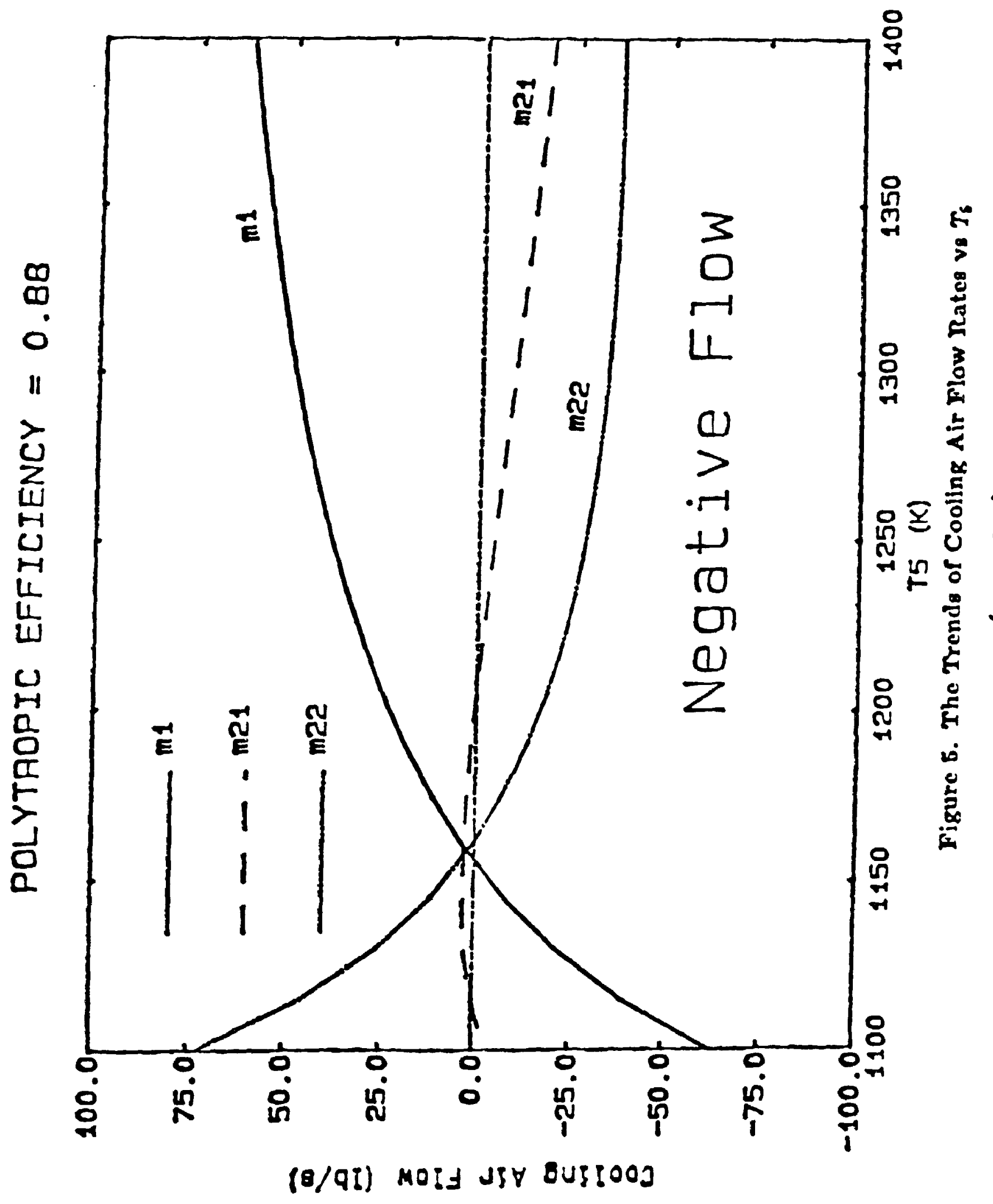




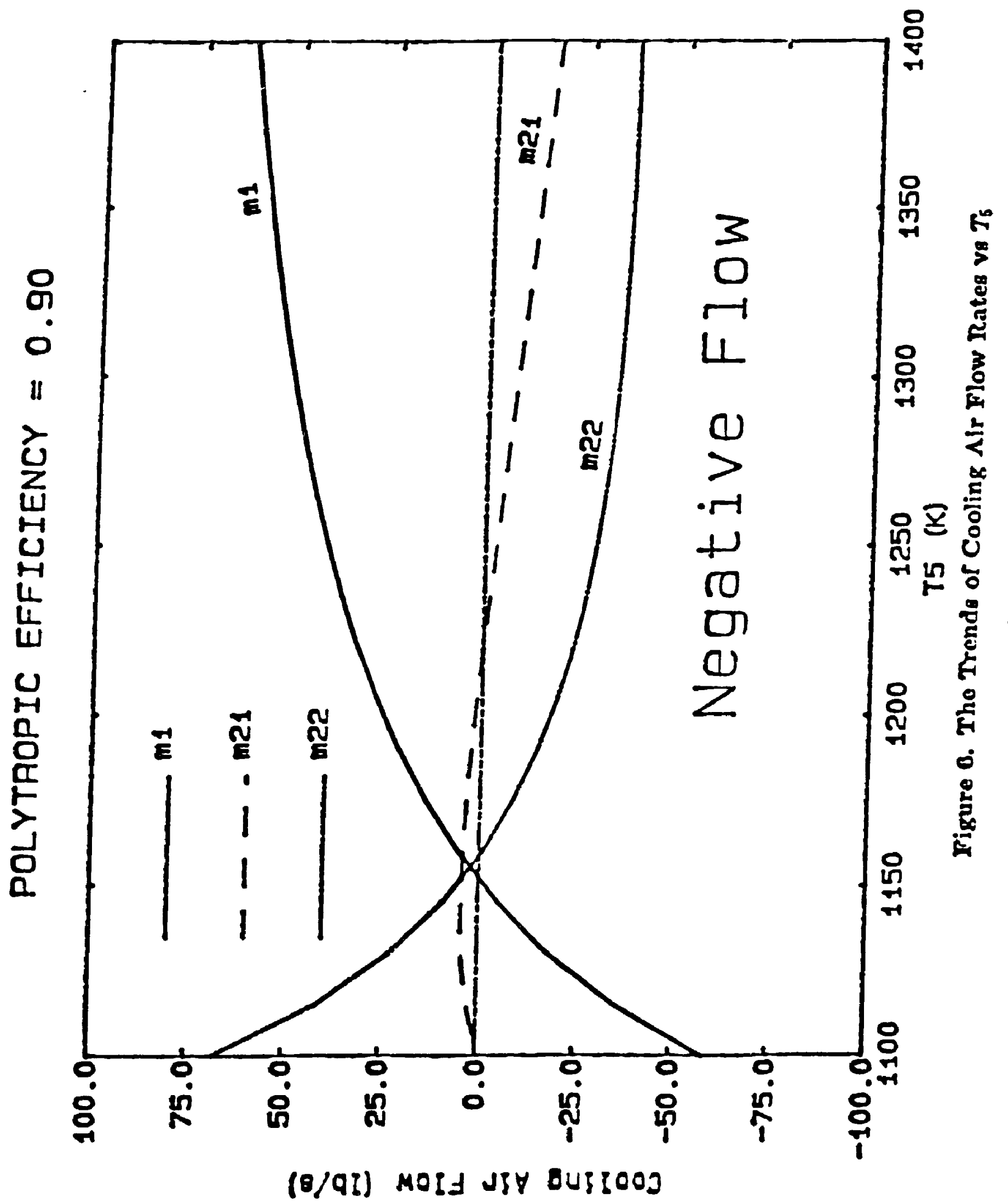




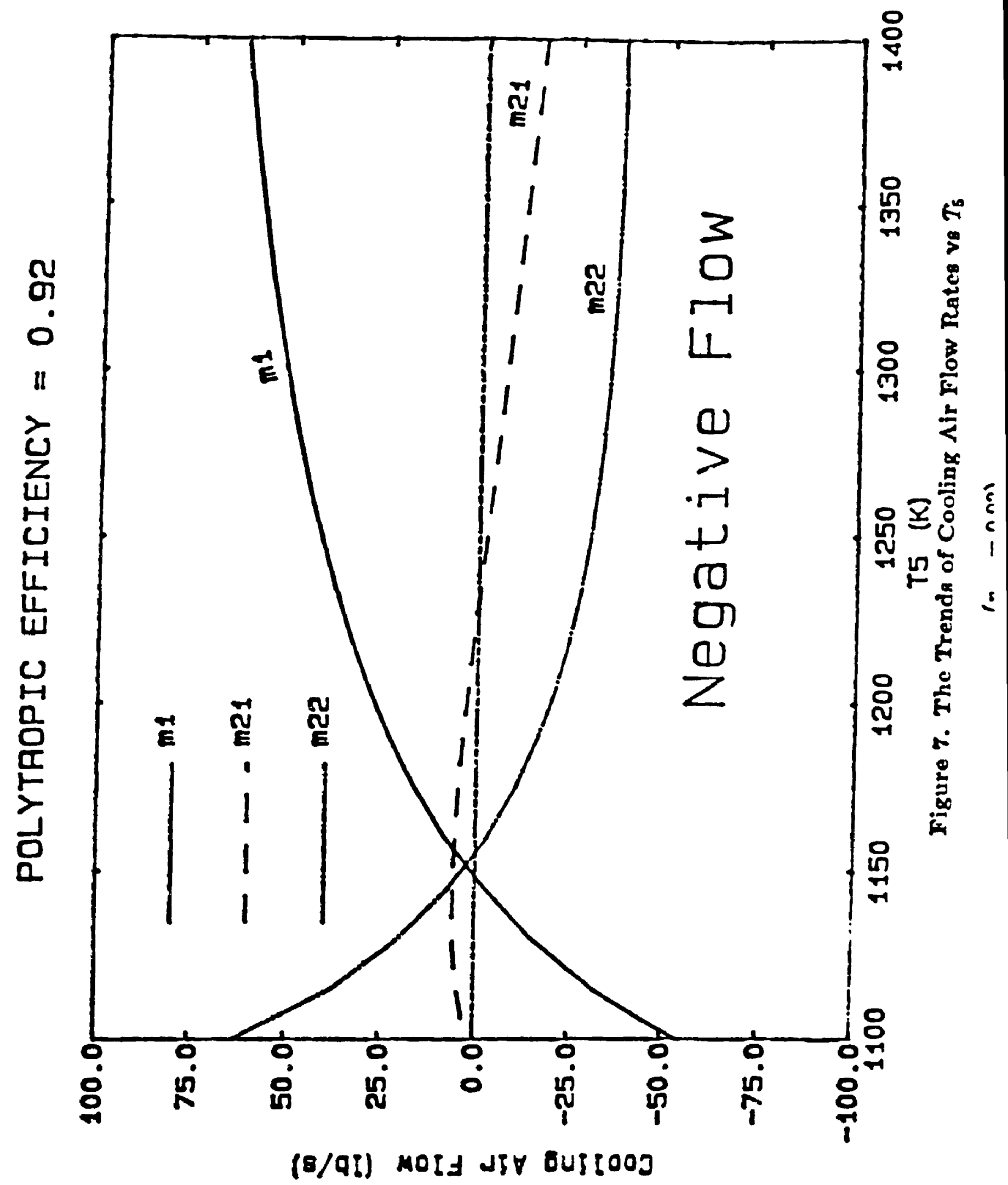




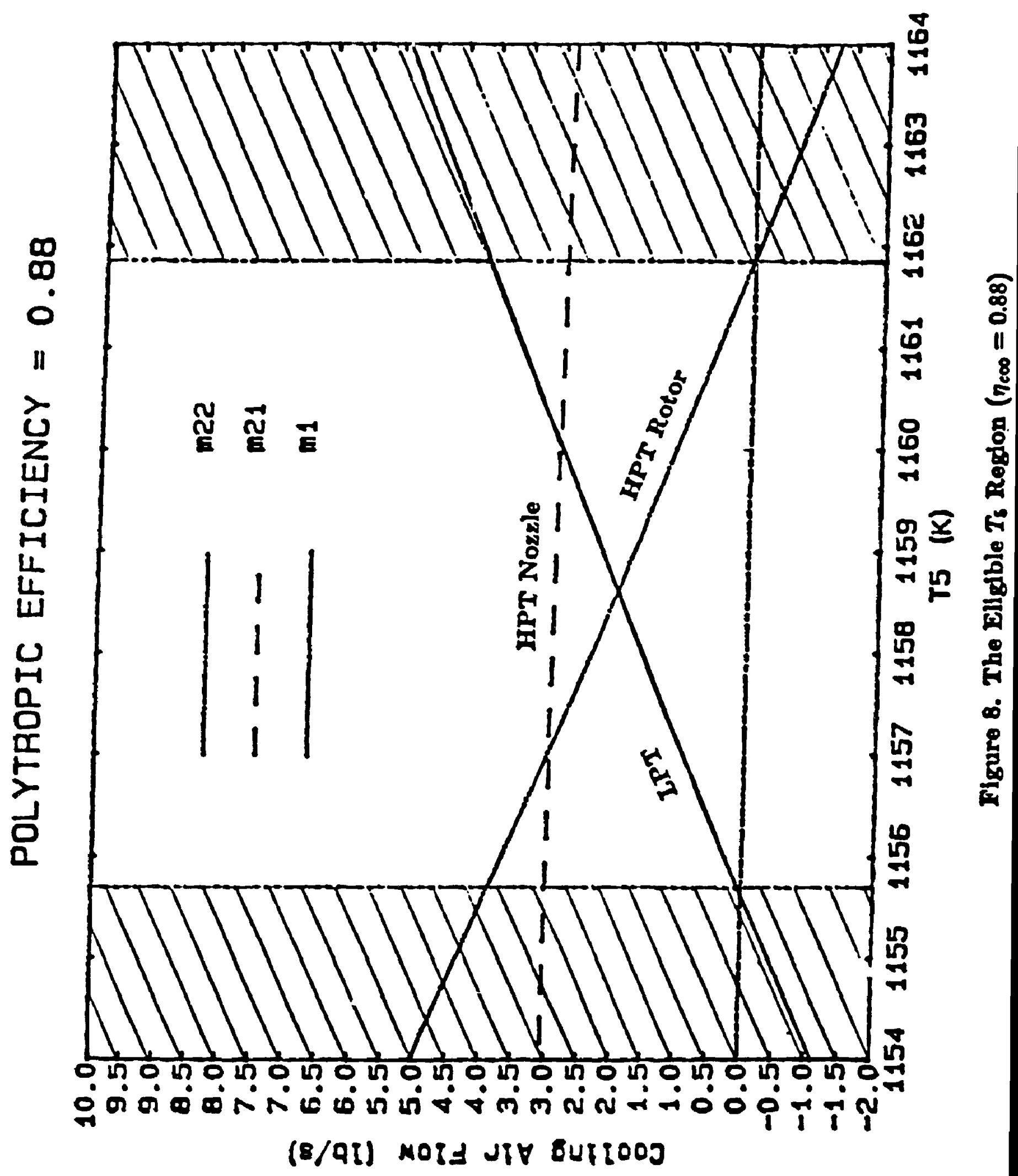




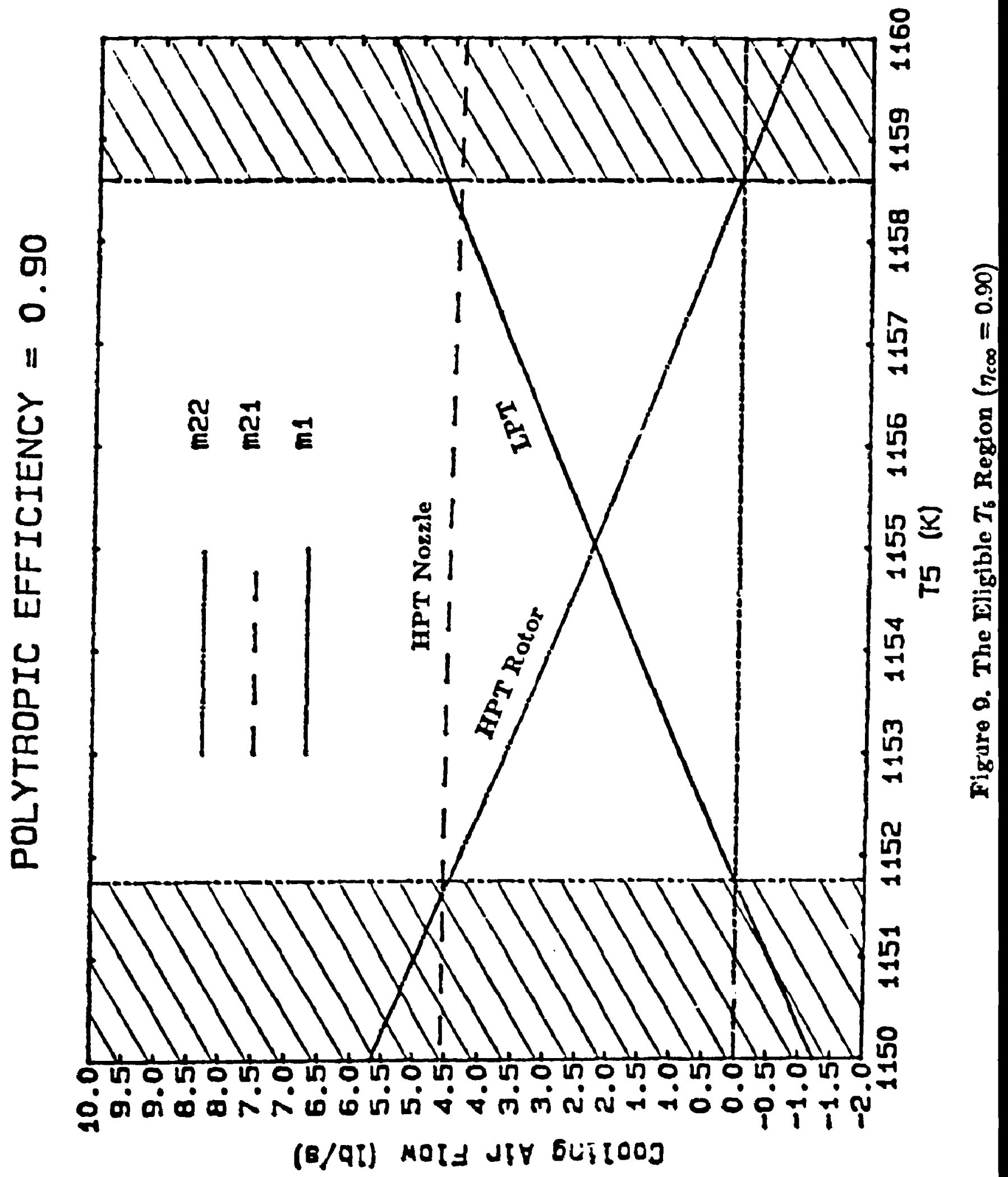




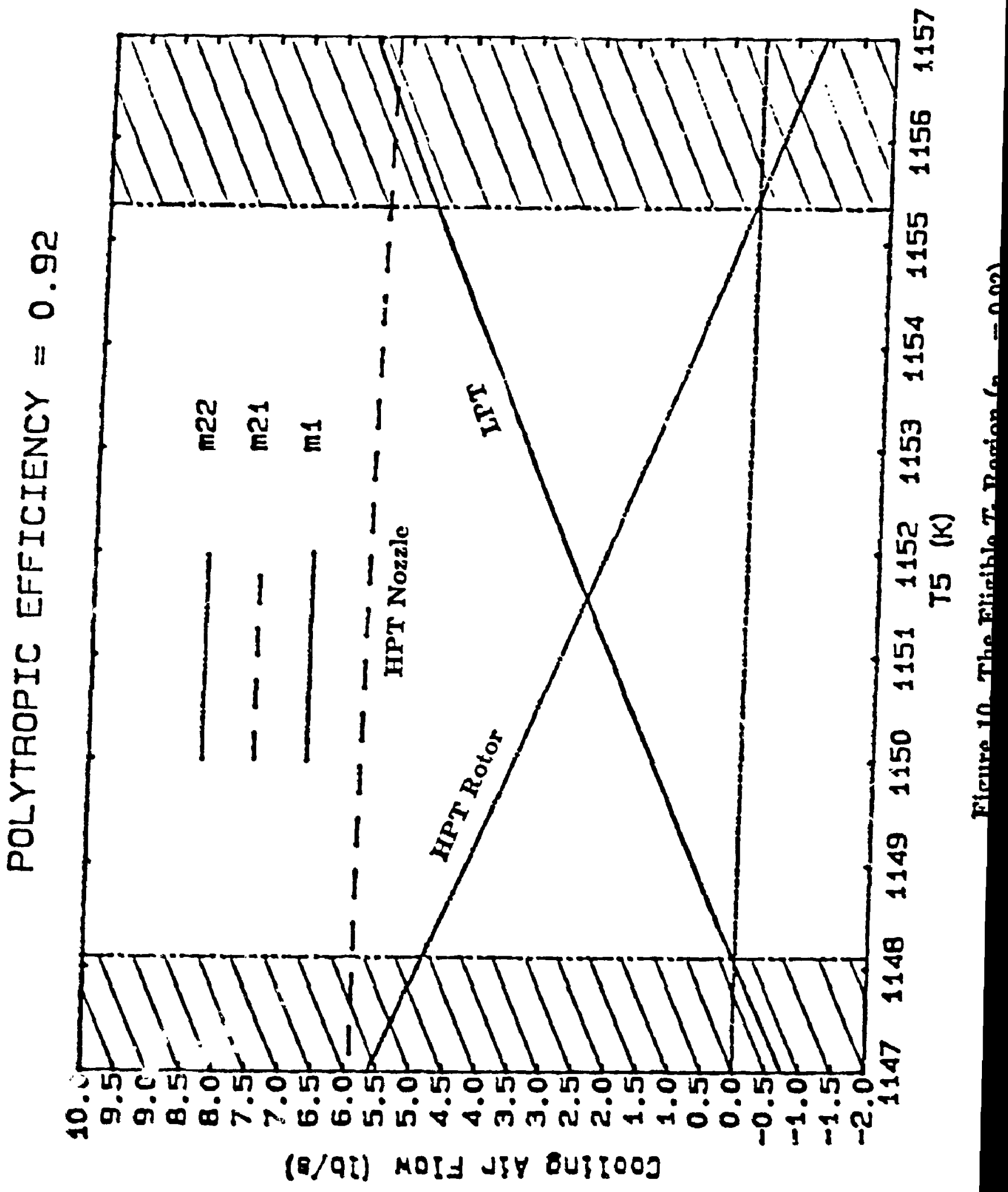




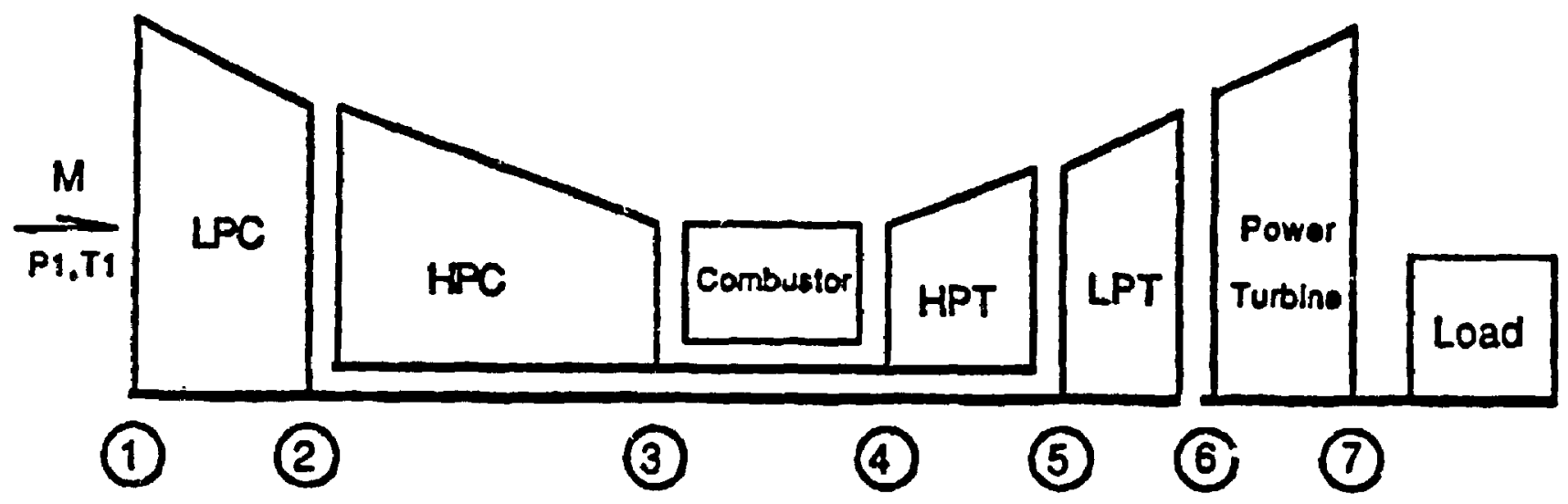

Figure 11. Twin-spool Land Based Gas Generator and Station Numbering System

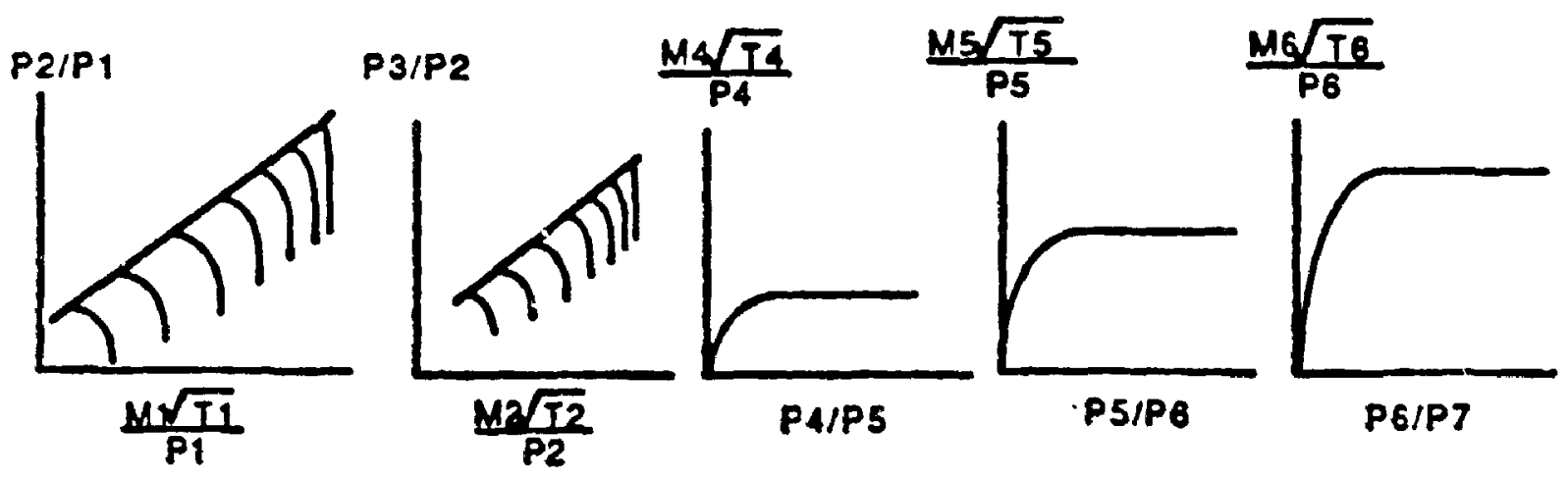

Figure 12. Component Charactaristics 


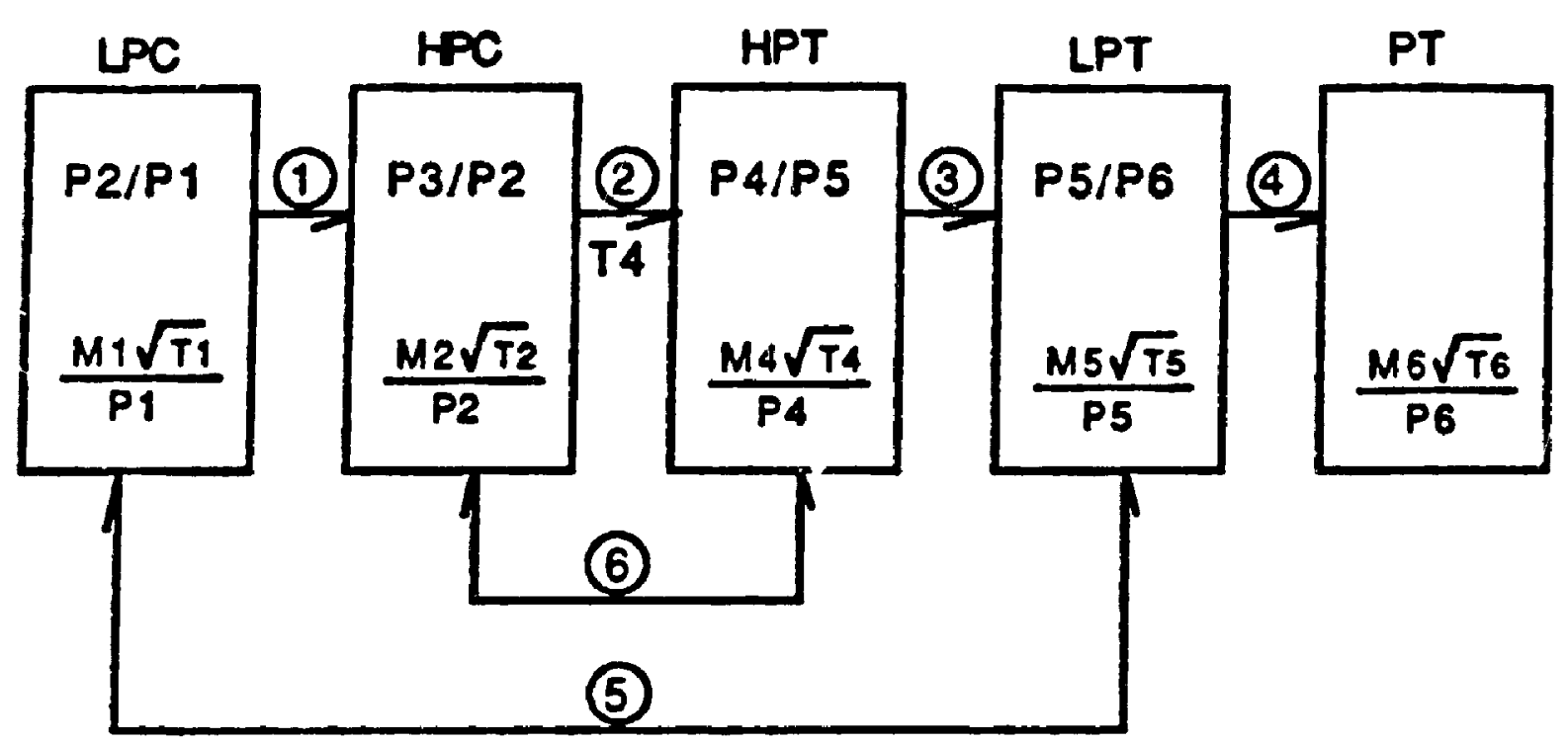

Figure 13. Relation of Component Matching Governing Equations and Unknowns

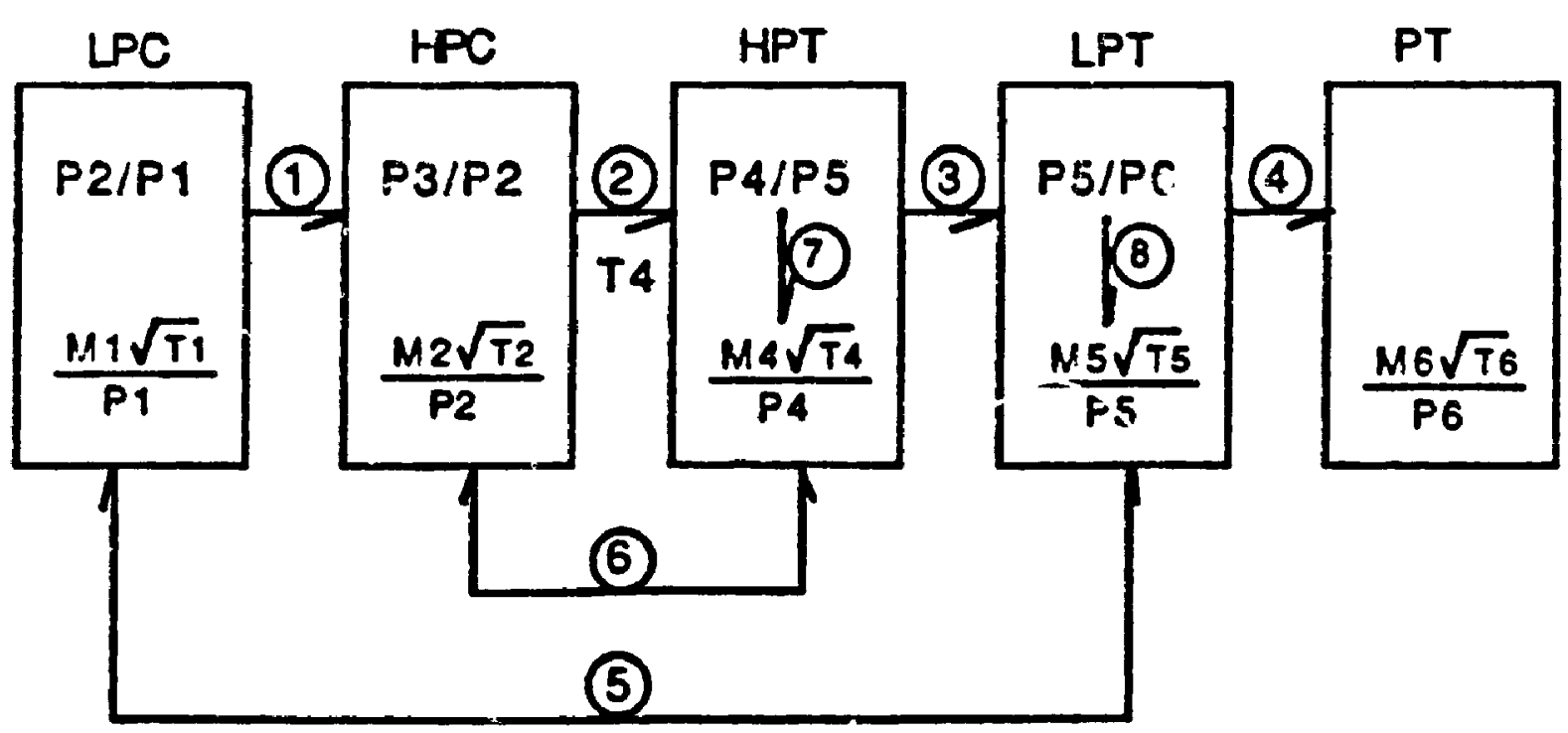

Figure 14. Modifed Equation and Unknown Relation 


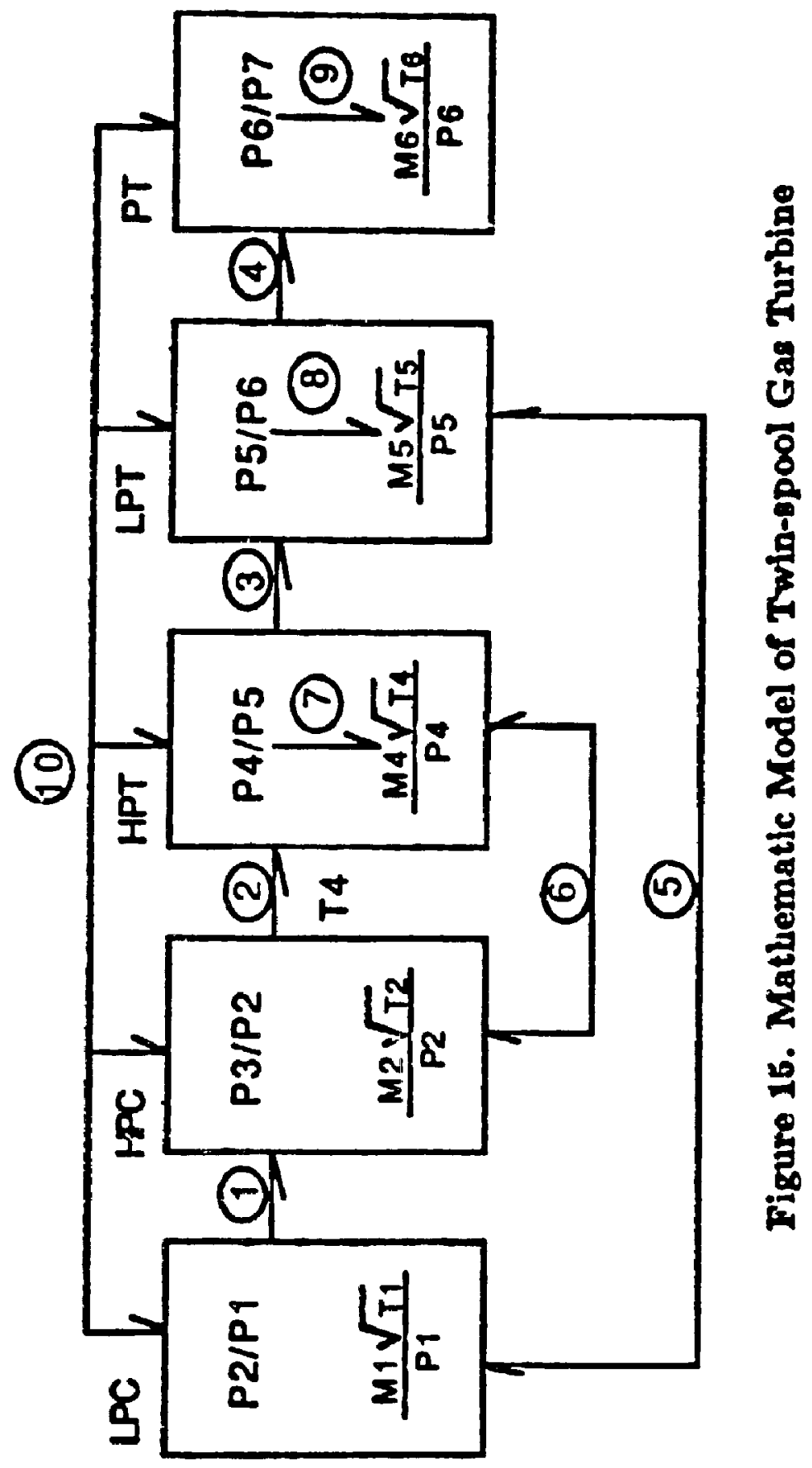



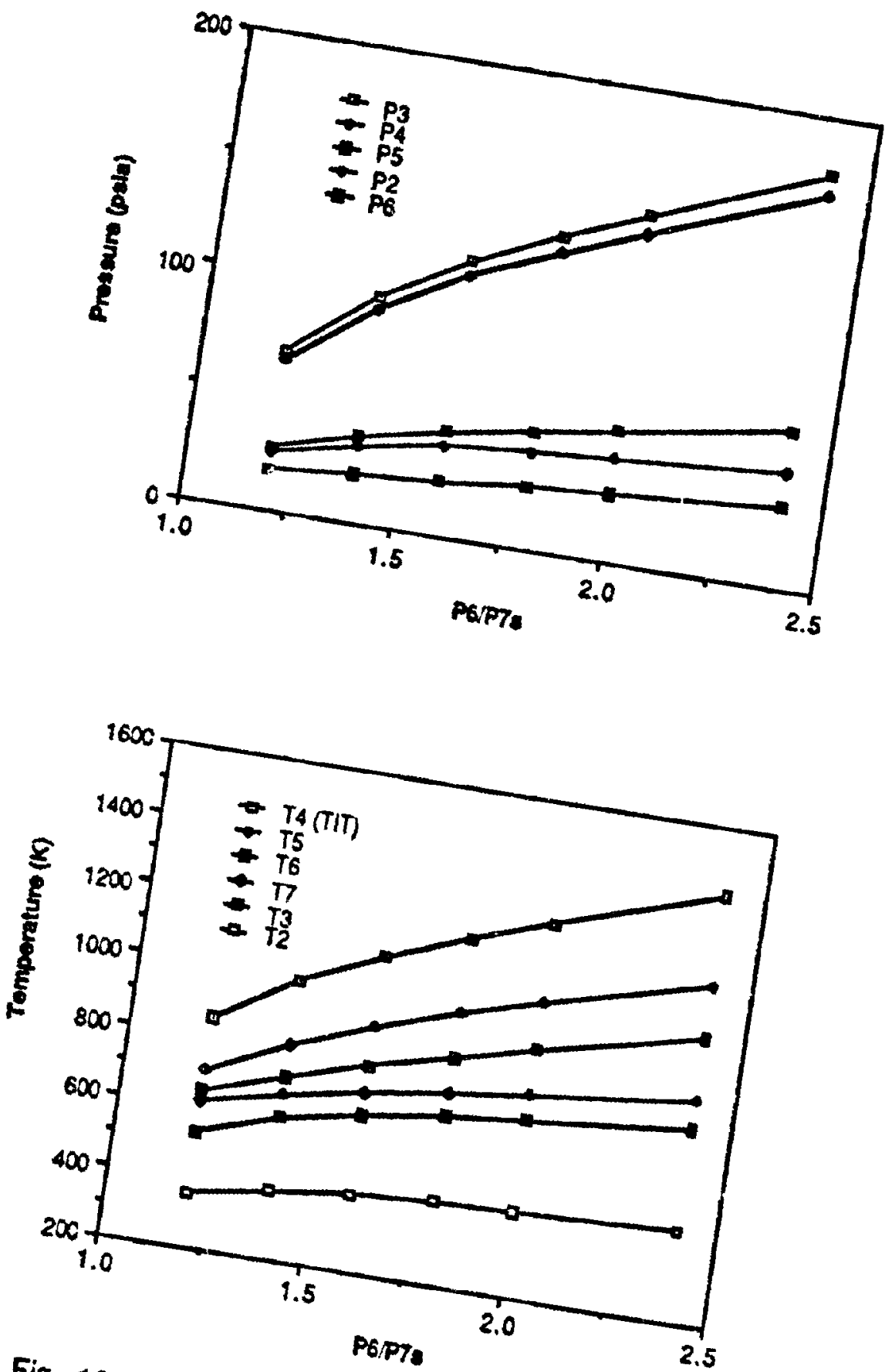

Fig. 16.1 : Relationships between P6/P7s and
Pressures and Temperatures 

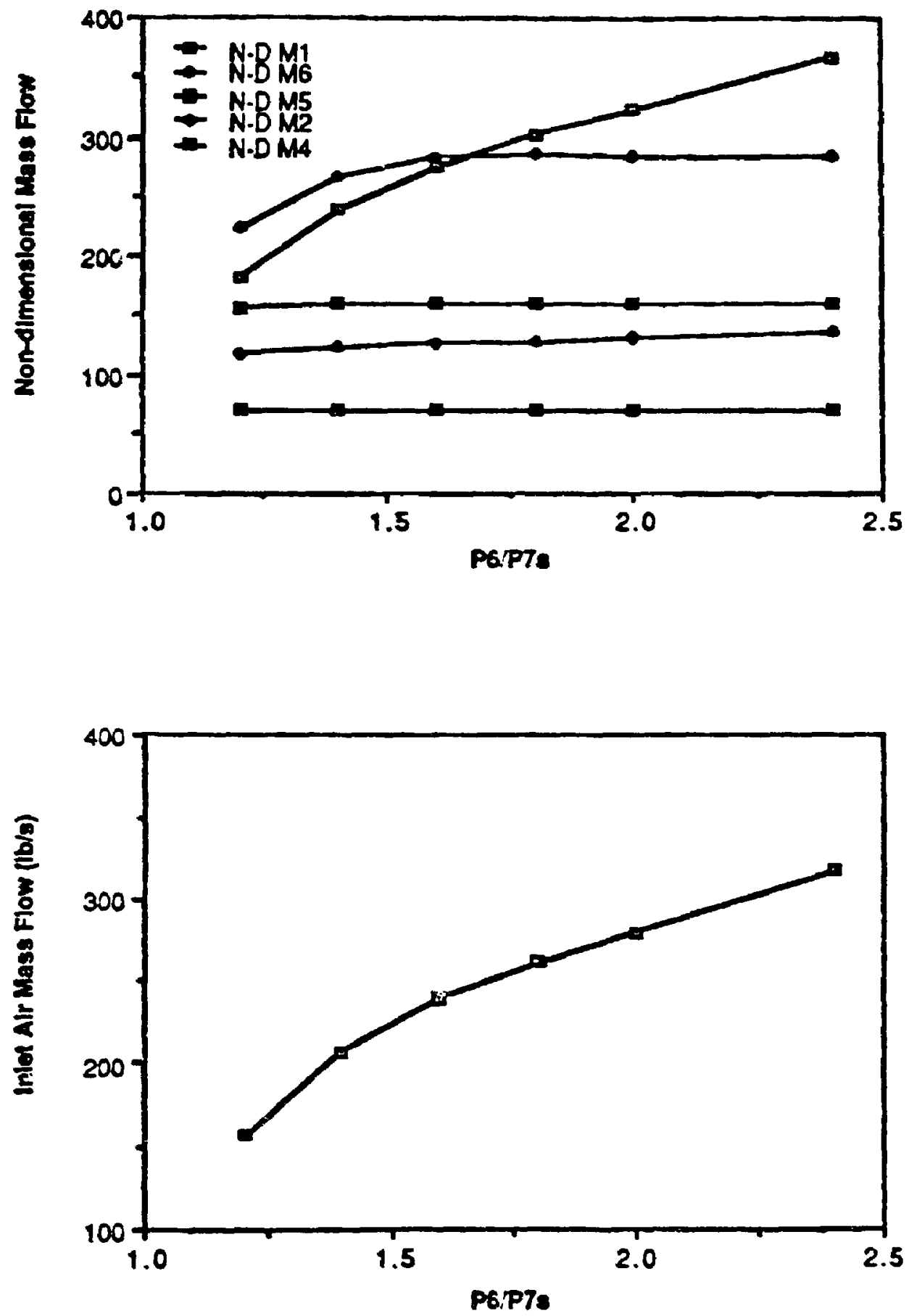

Fig 16-2 : Relationships between P6/P7s and Non-dimensional Mass Flow and Inlet Air Flow Rates 


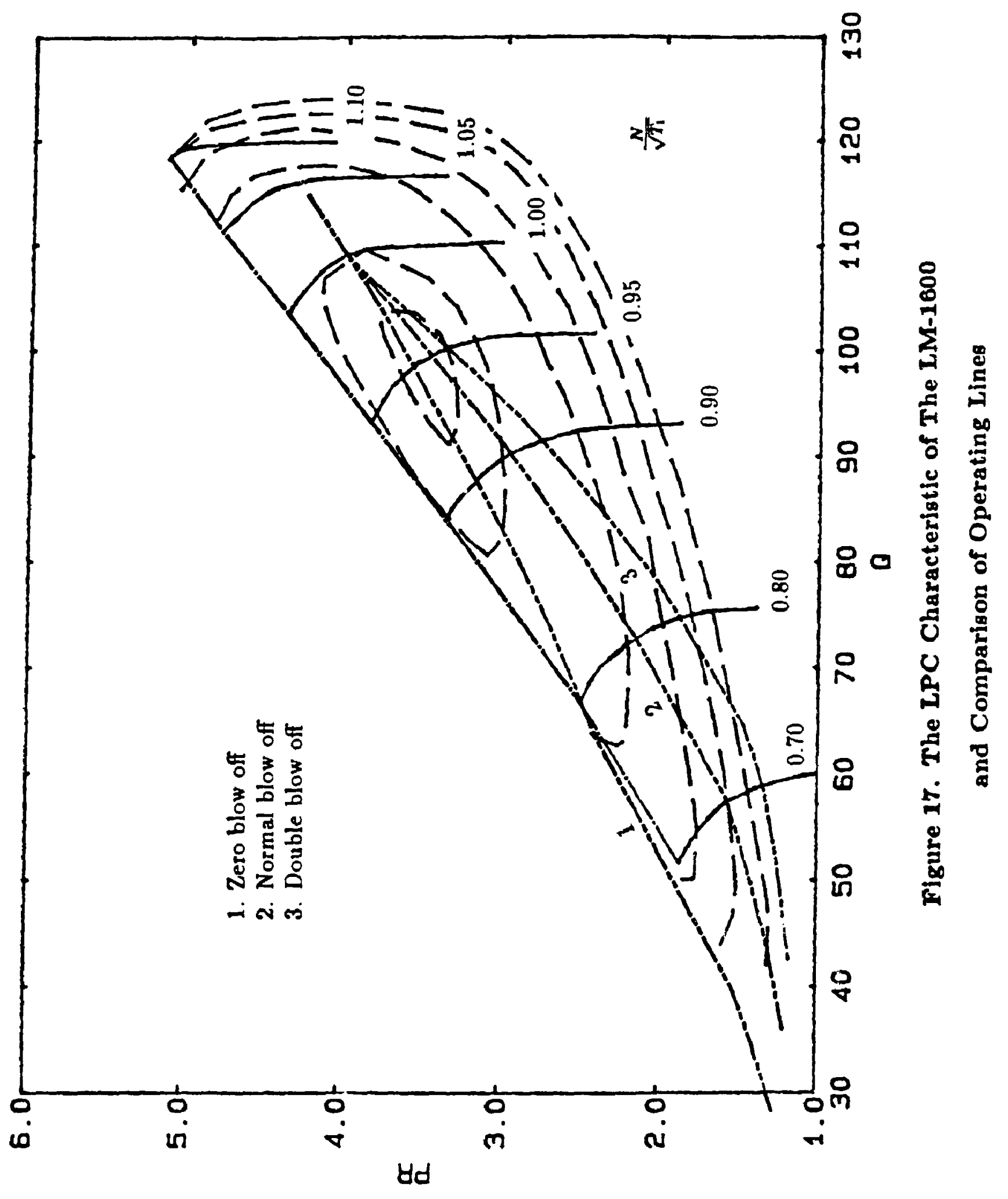




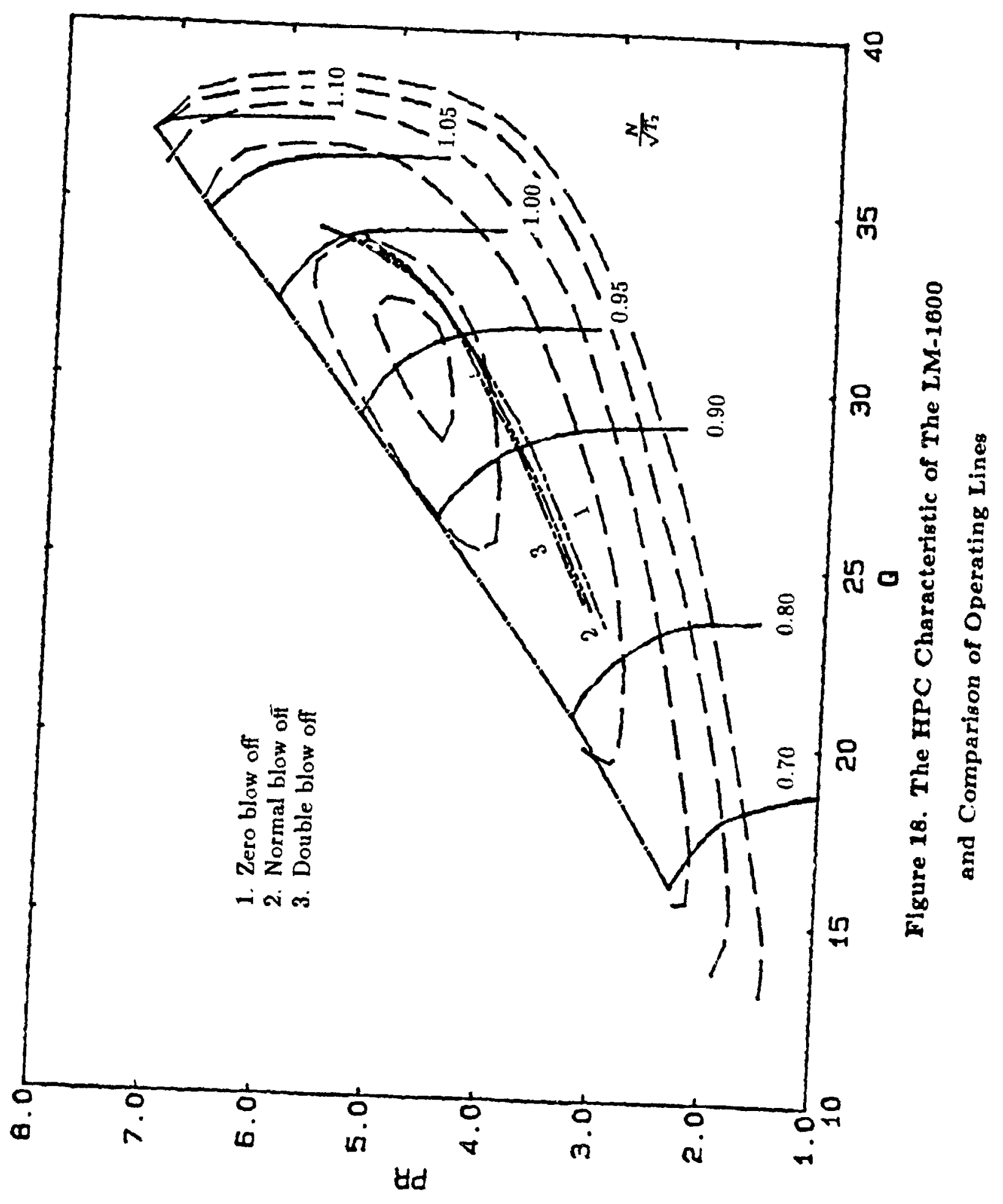




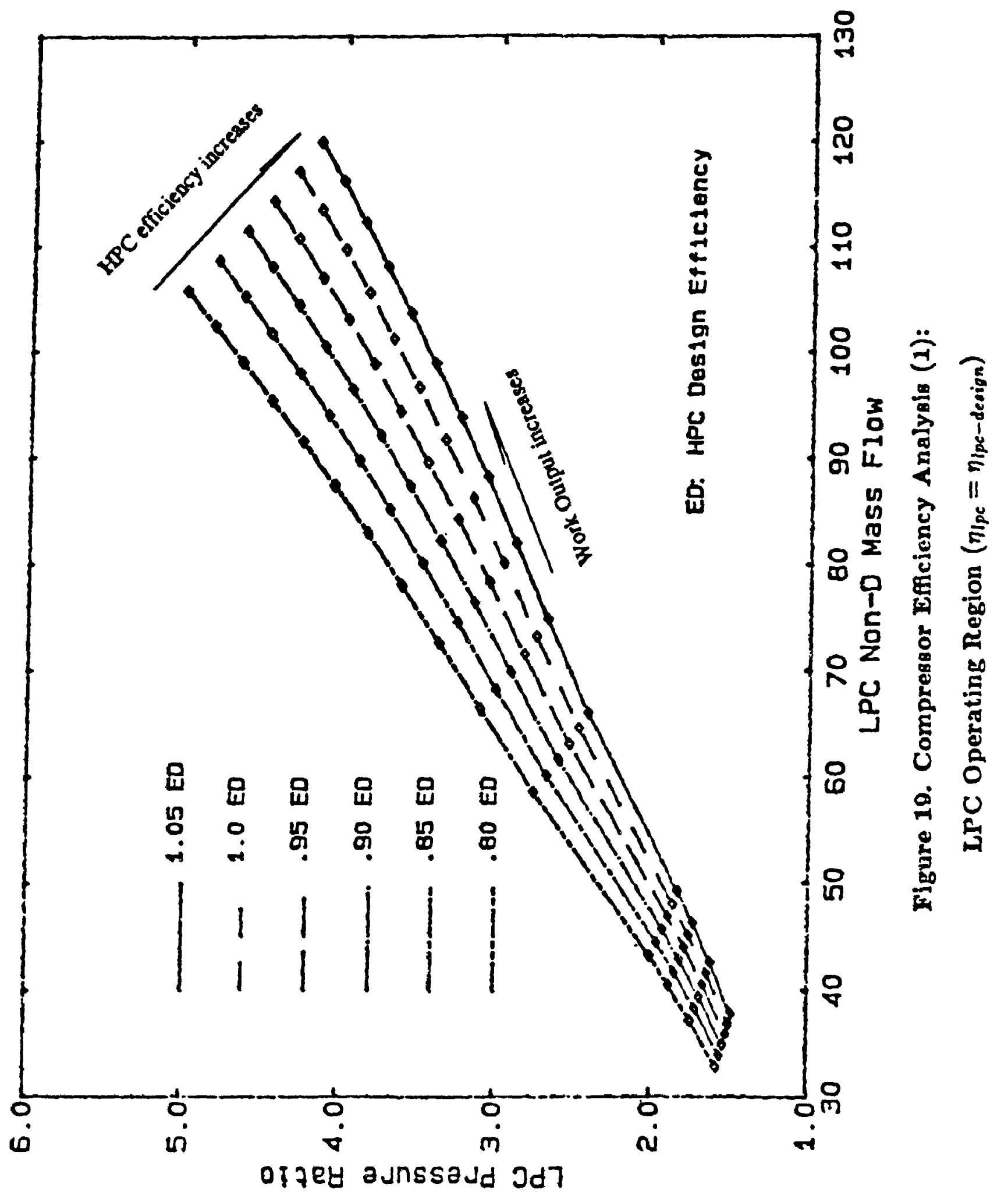




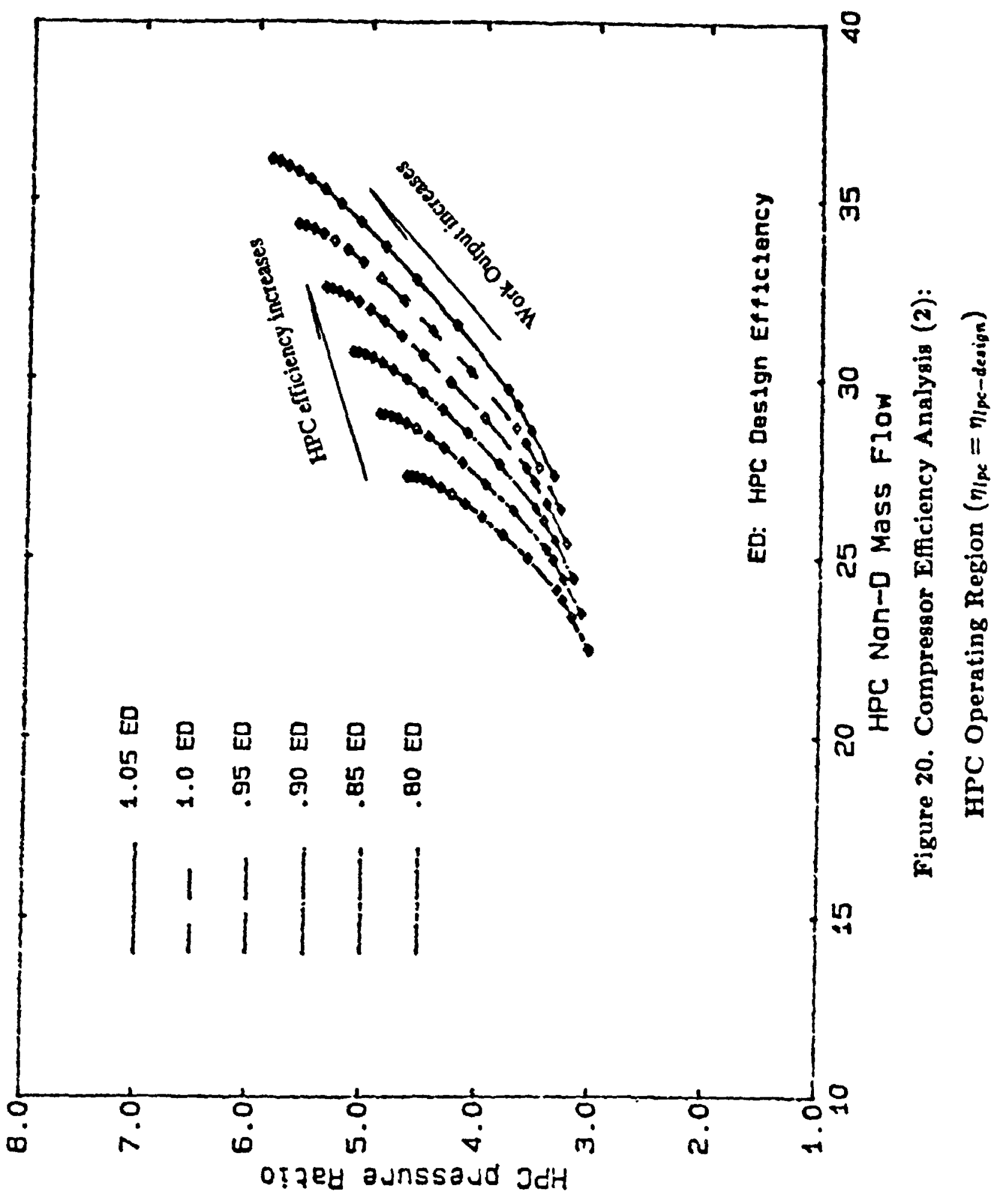




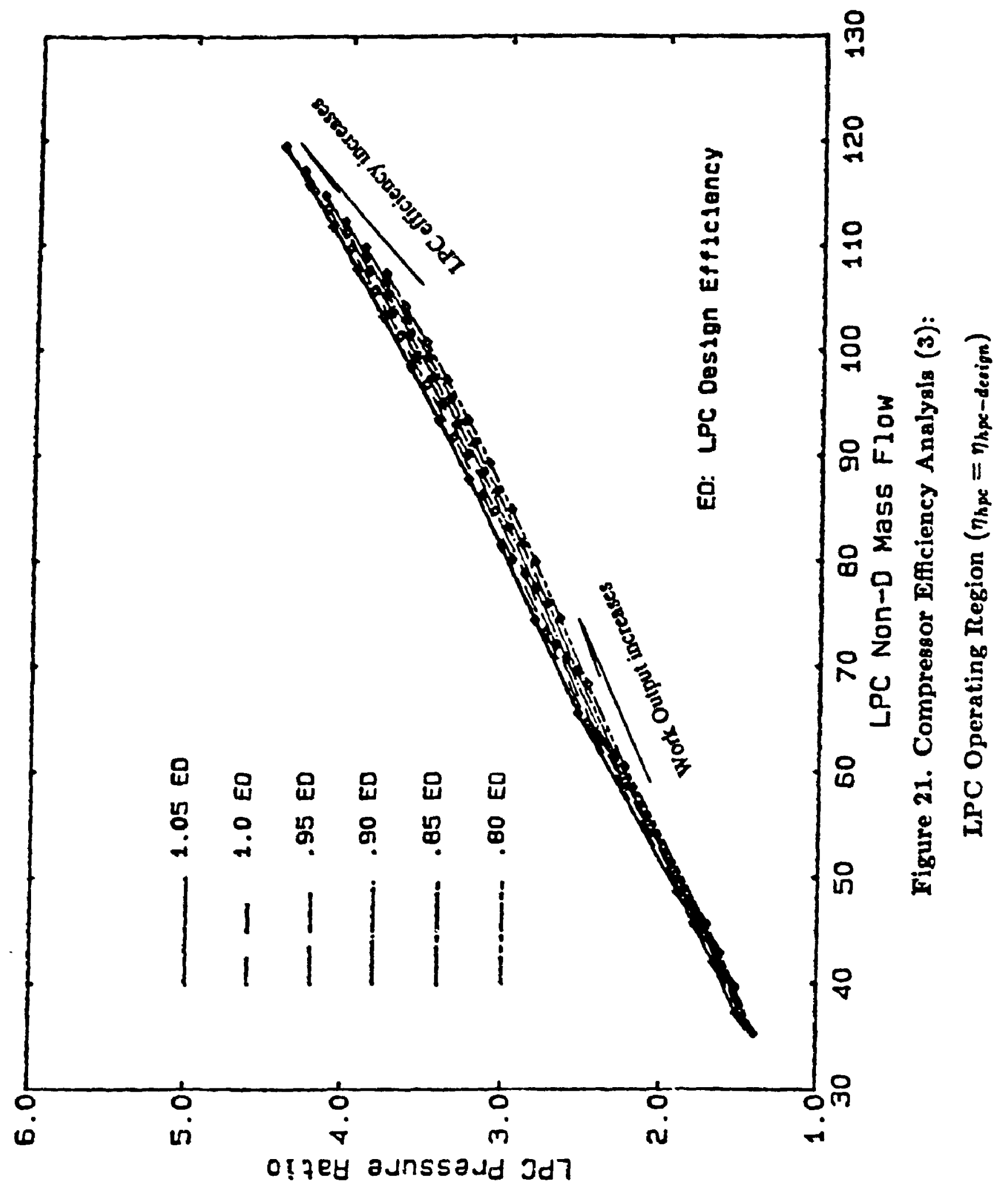




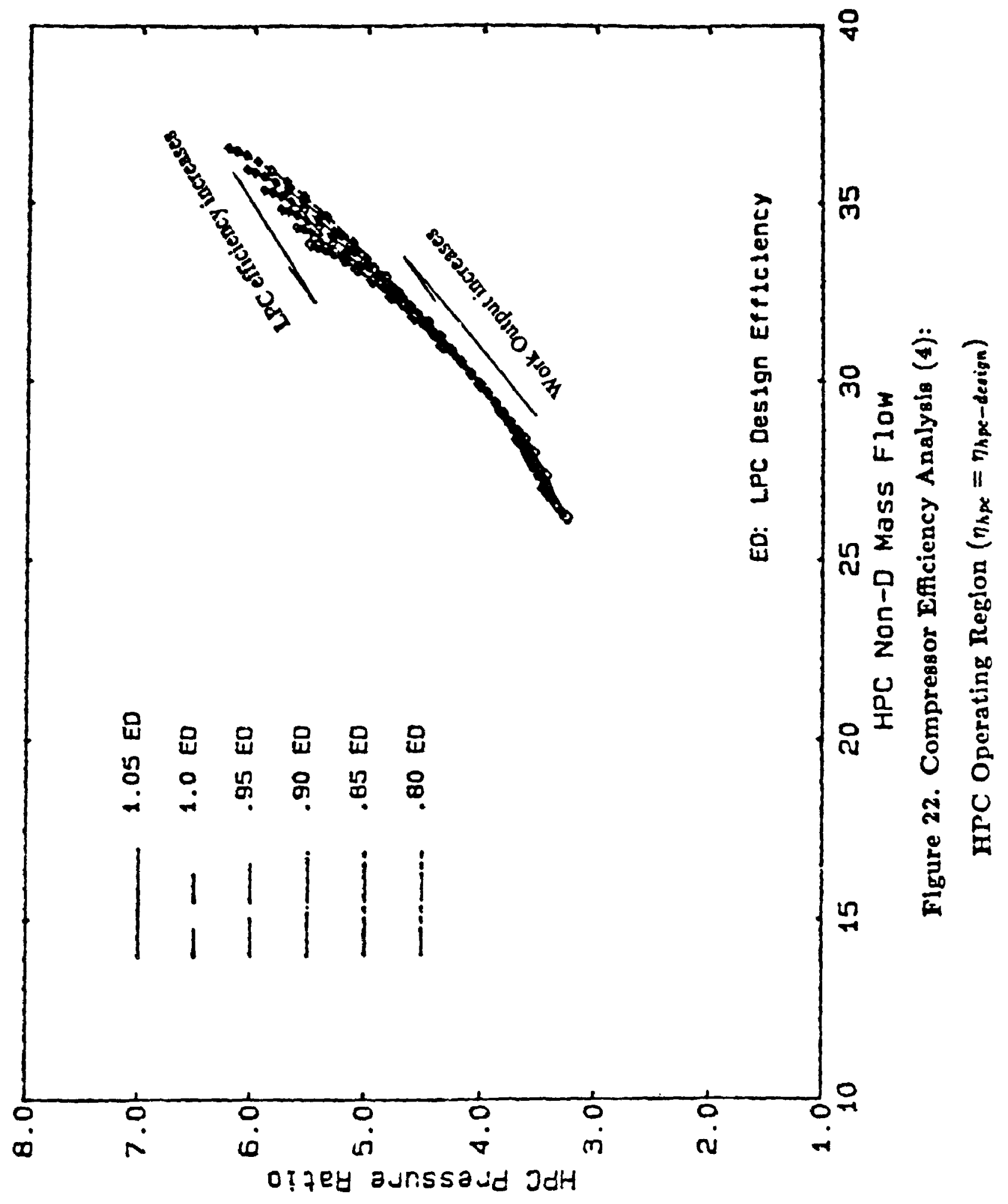




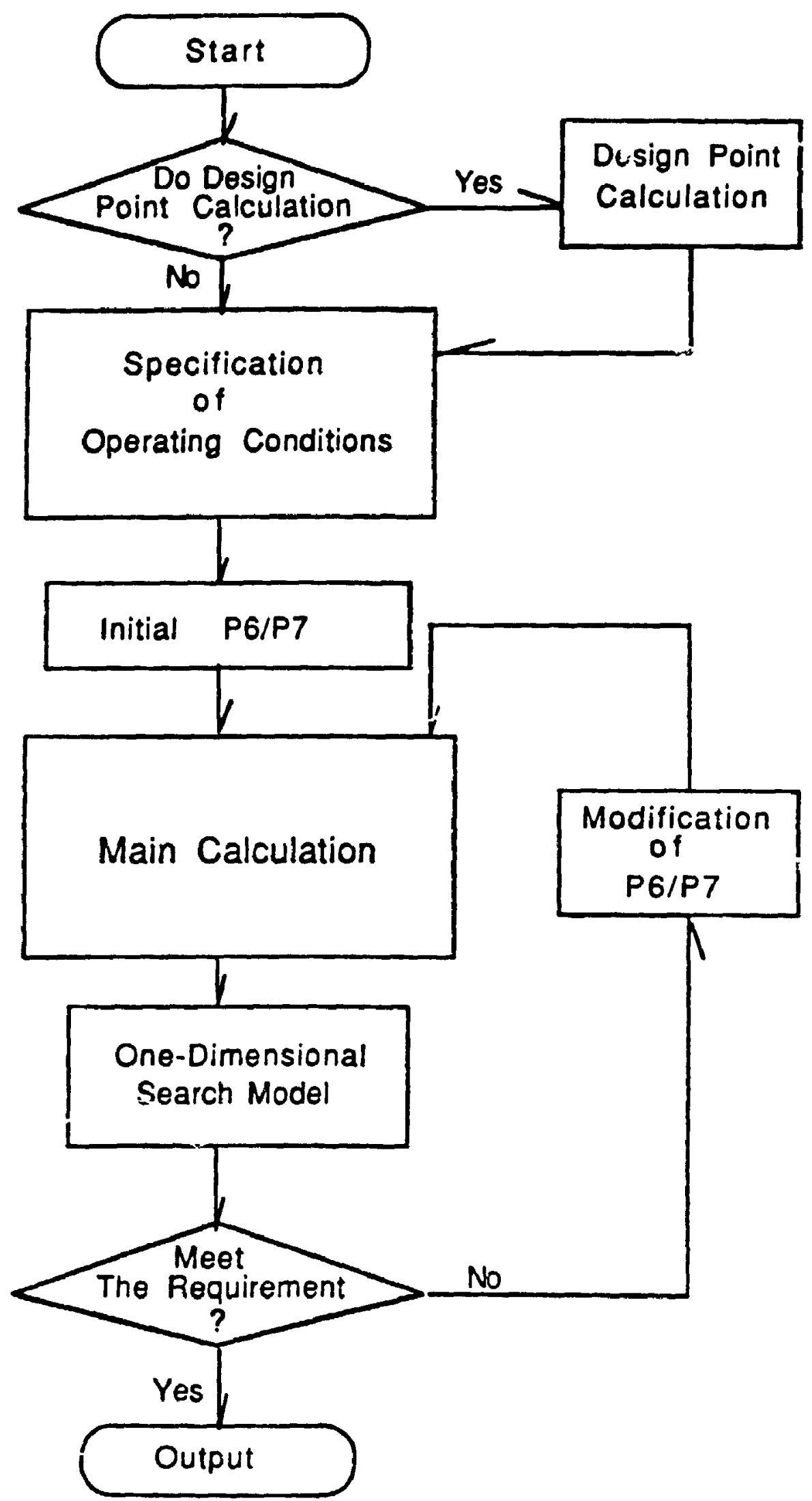

Figure 23. Overall Program Structure of LM16 
(a)

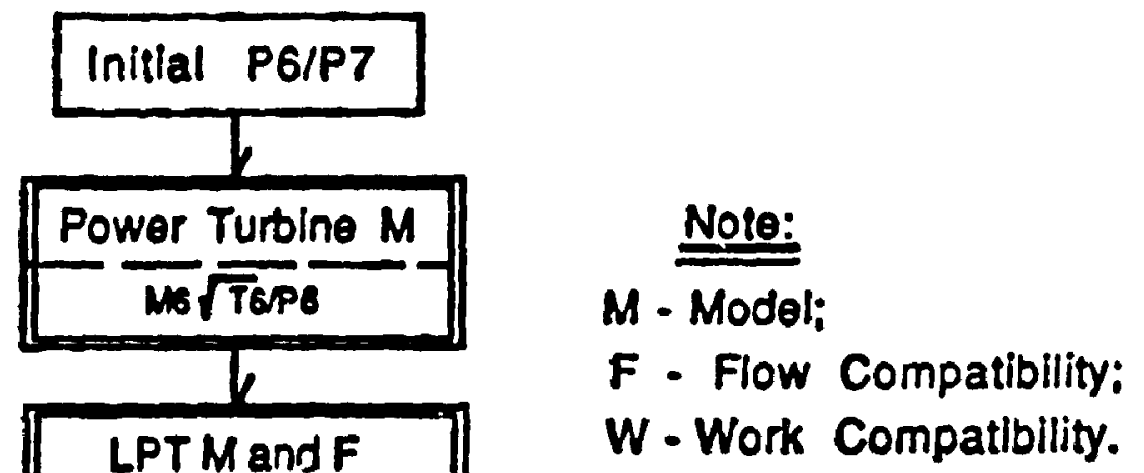

(b)

(c)
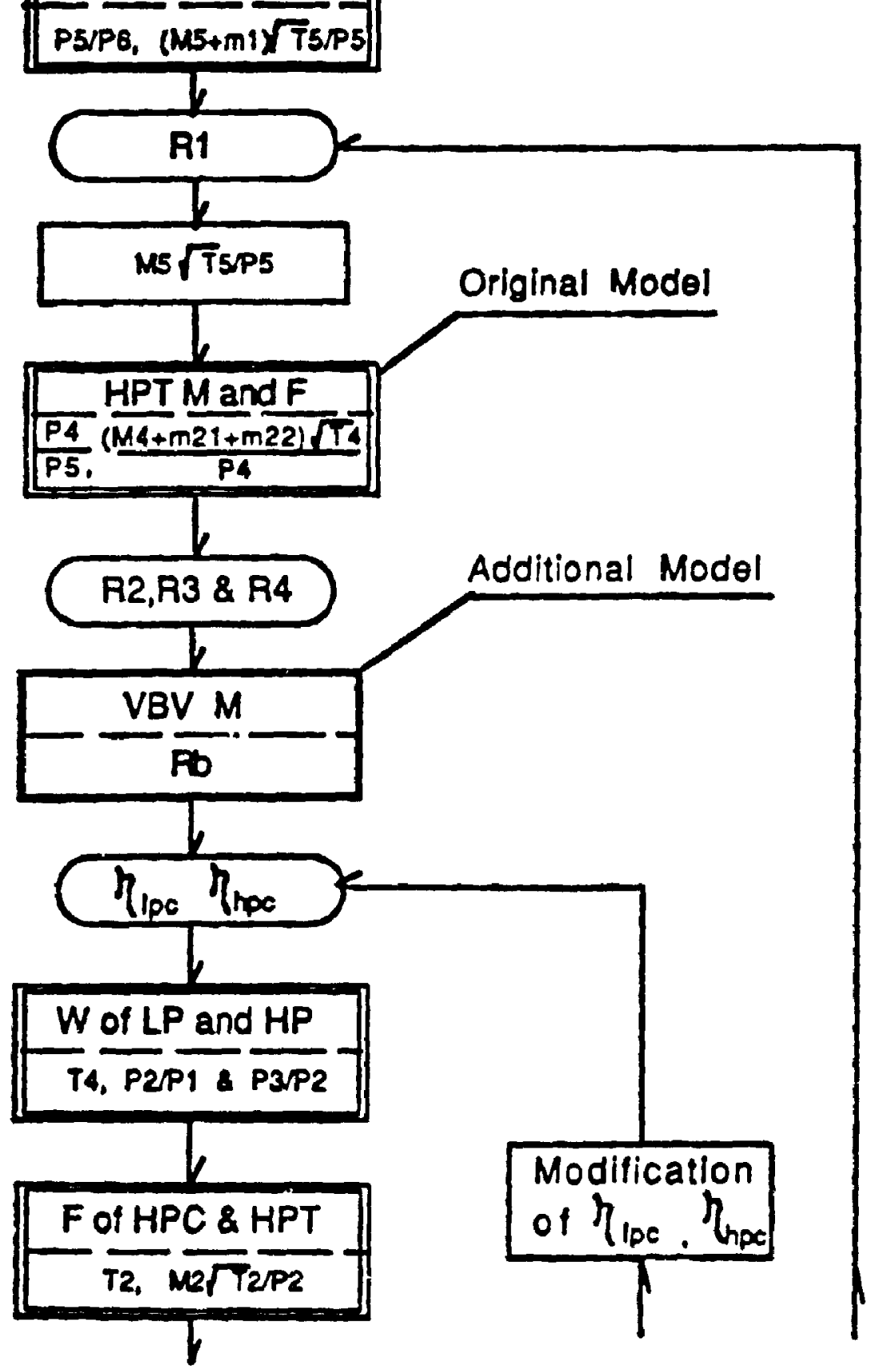

(d)

( to be continued)

132 
(e)

(i)

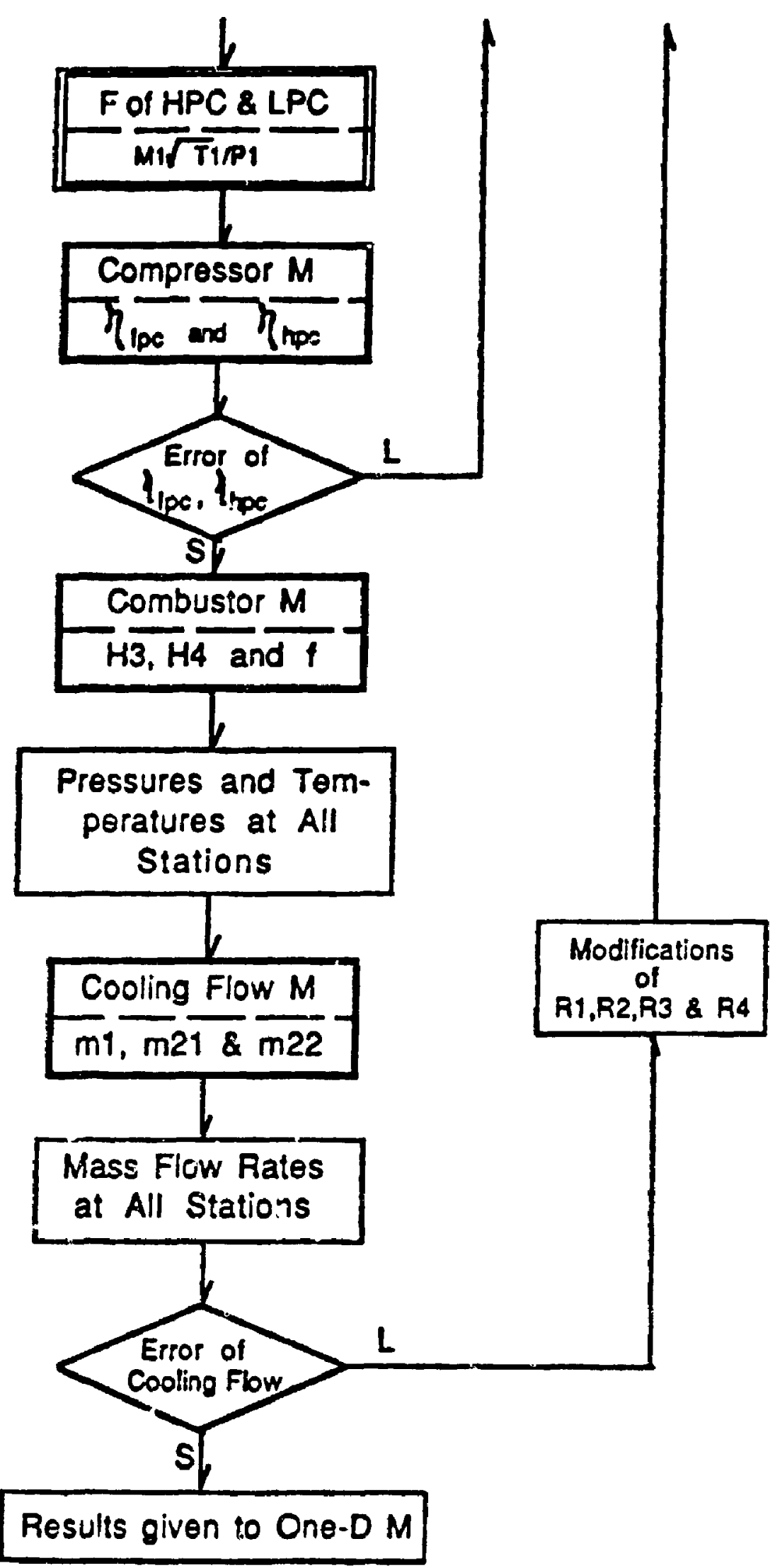

Figure 24. Flow Path of The Maln Calculation of LM16 


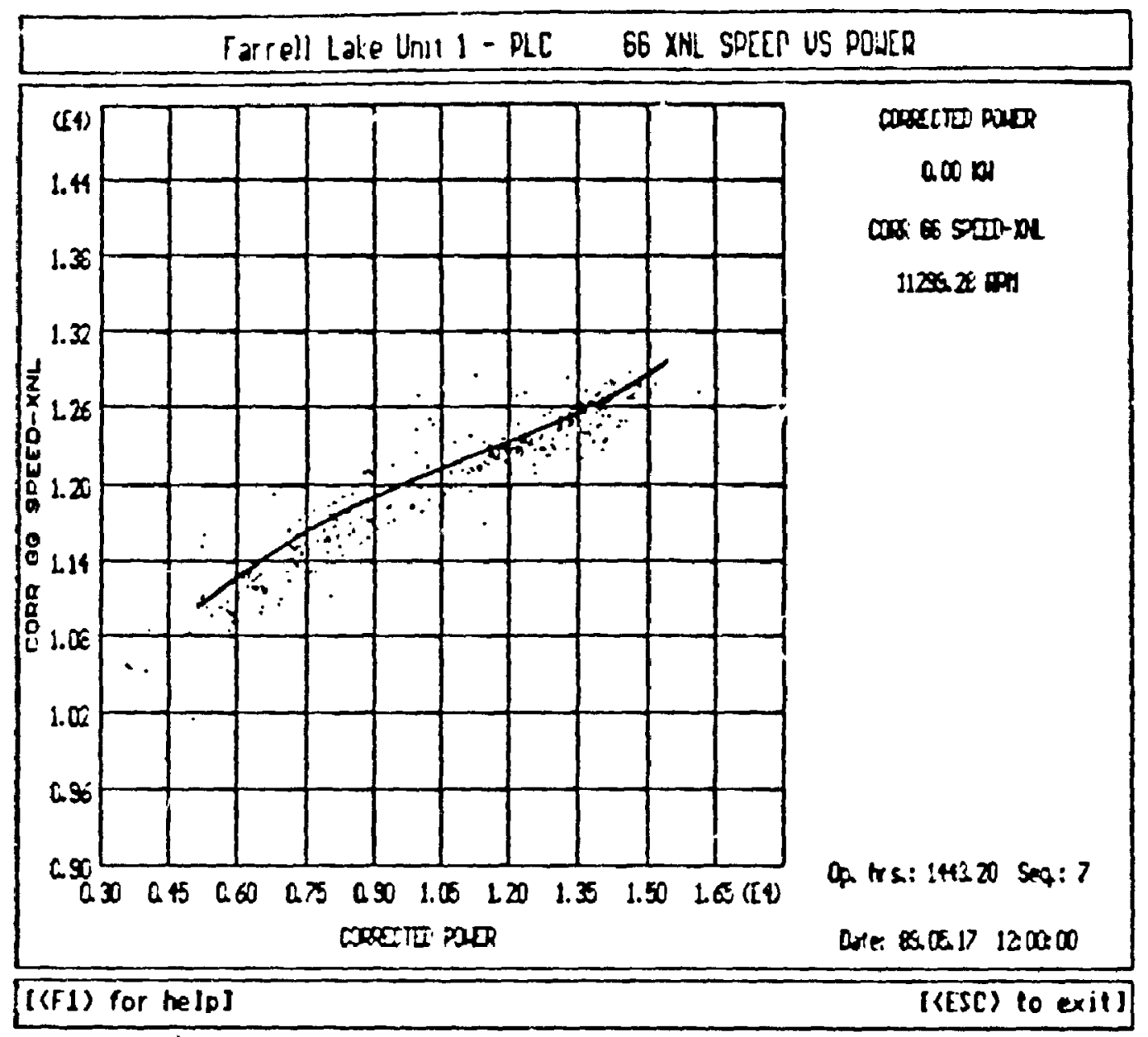

Figure 25. Comparison between Model Prediction and Fieid Measurement ( $N_{l}$ vs $W_{\text {out }}$ ) 


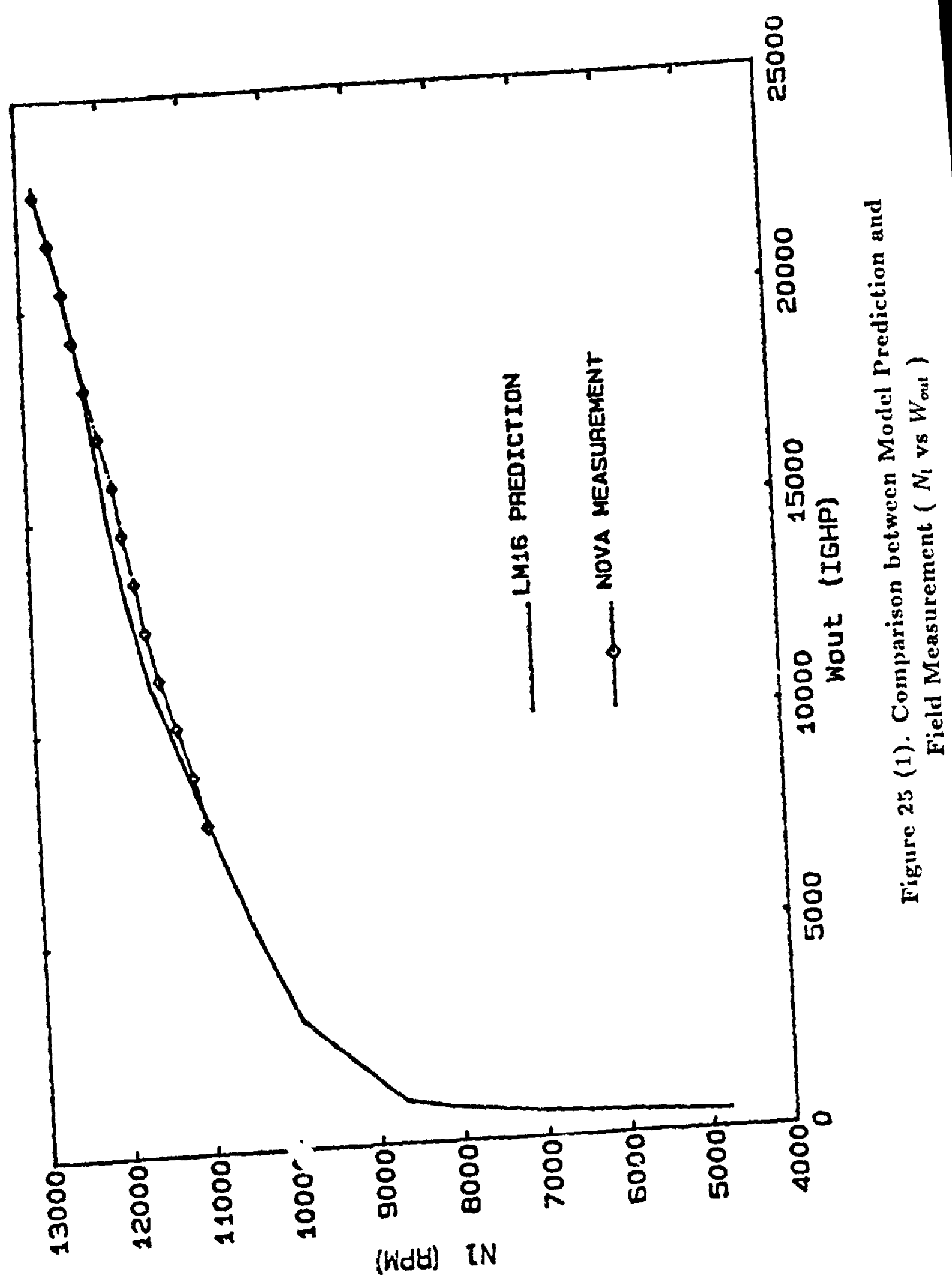




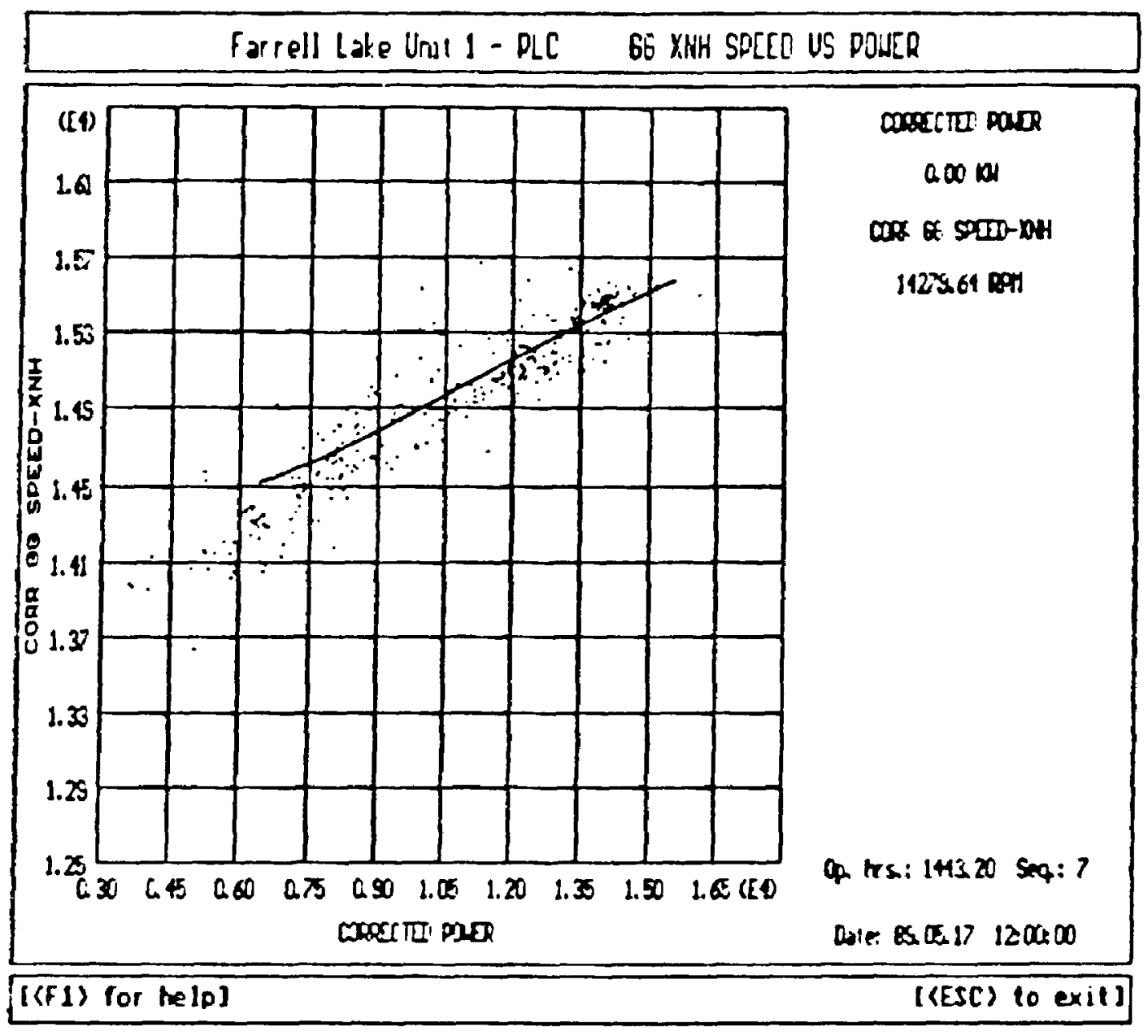

Figure 26. Comparisc a between Model Prediction and Field Measurement ( $N_{h}$ vs $W_{\text {out }}$ ) 


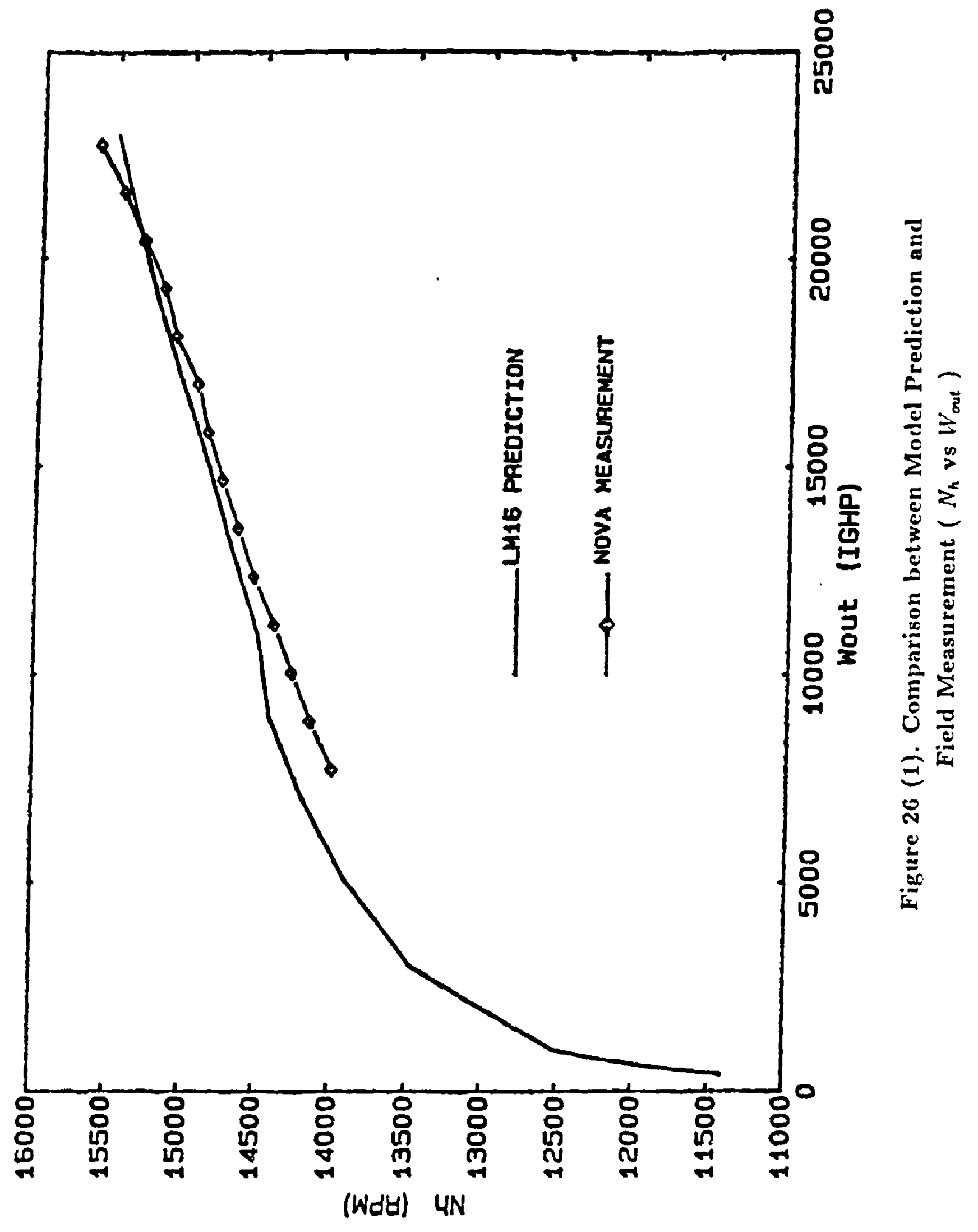




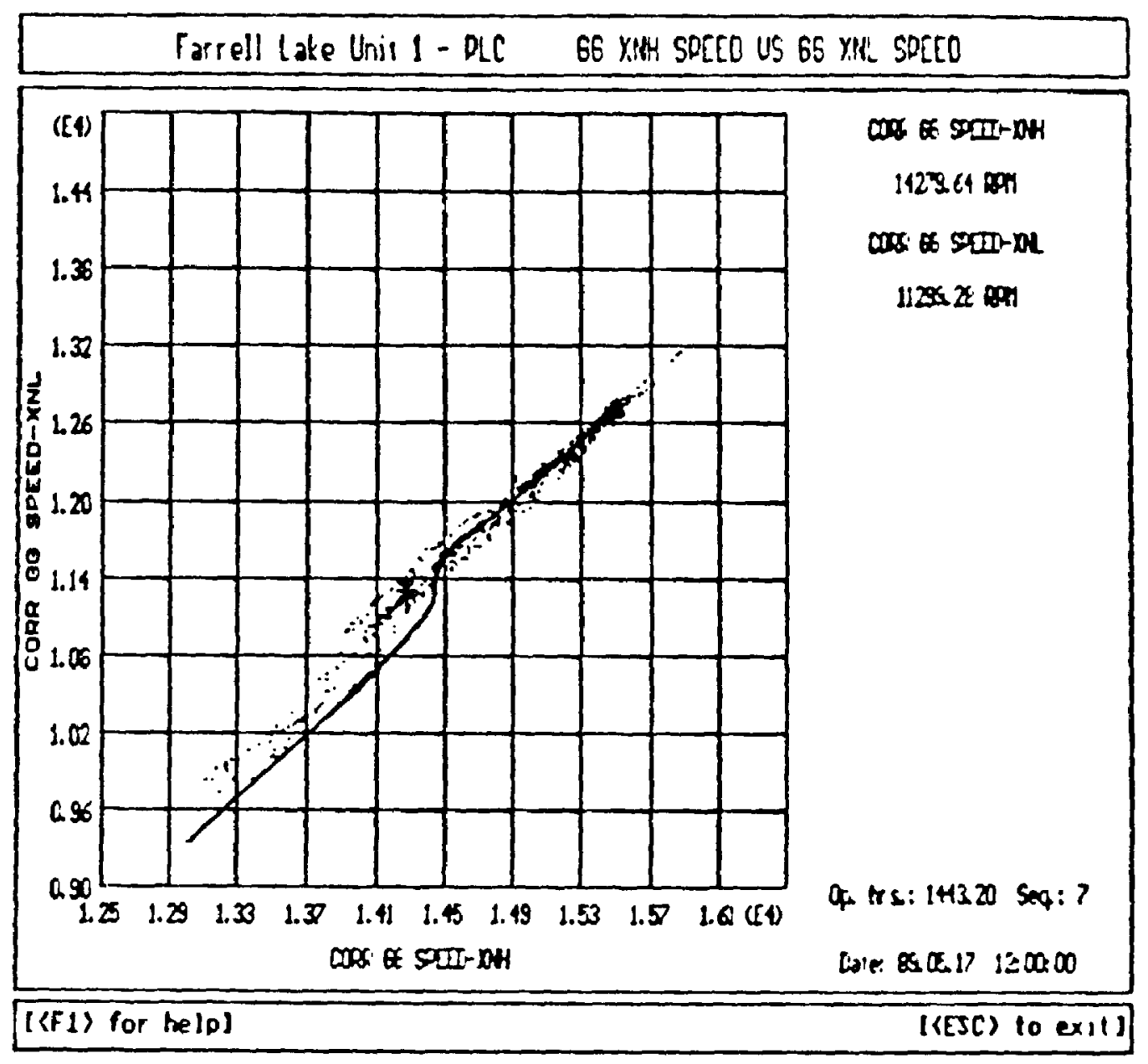

Figure 27. Comparison between Model Prediction and Field Measurement ( $V_{l}$ vs $N_{k}$ ) 


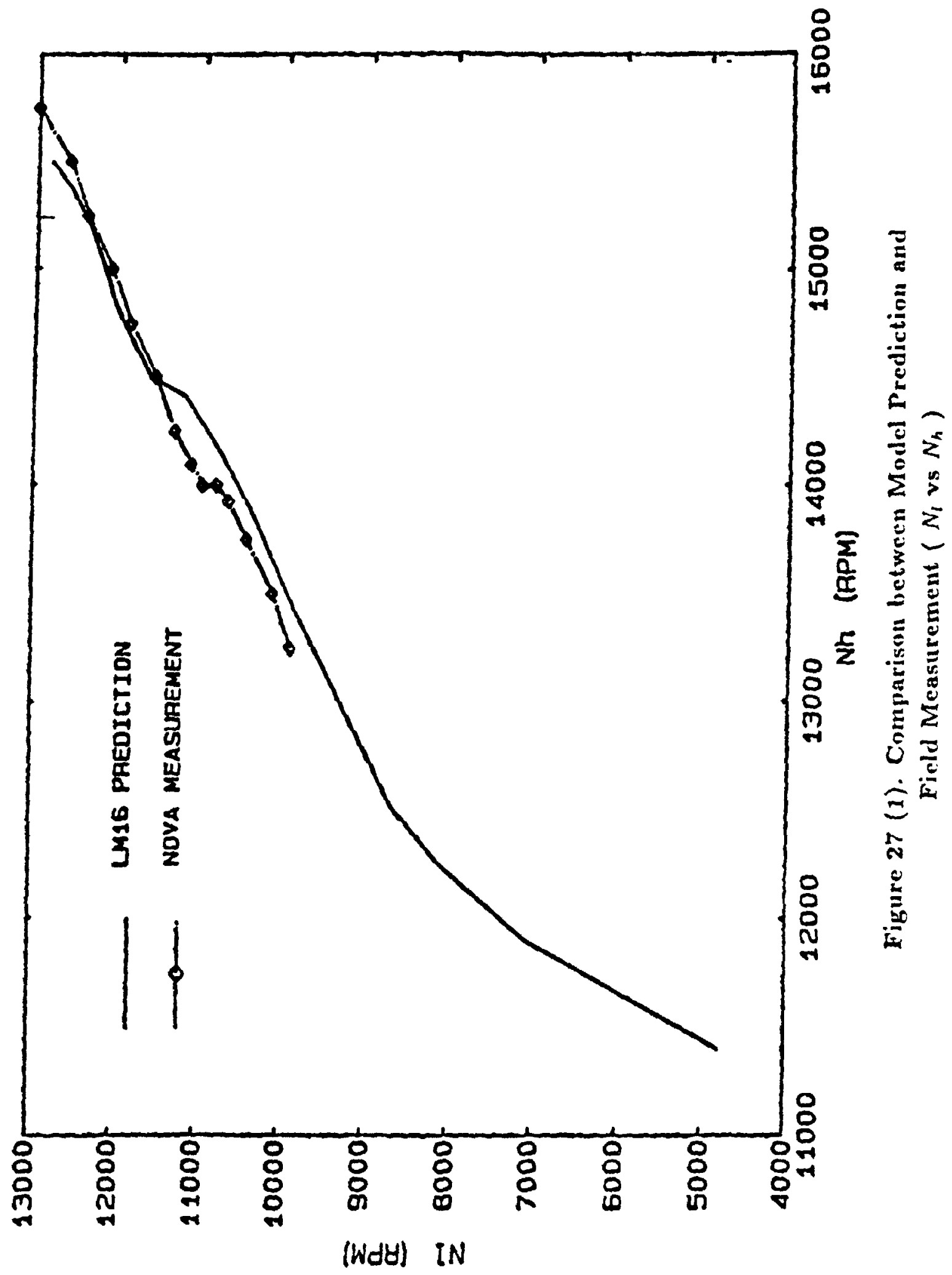




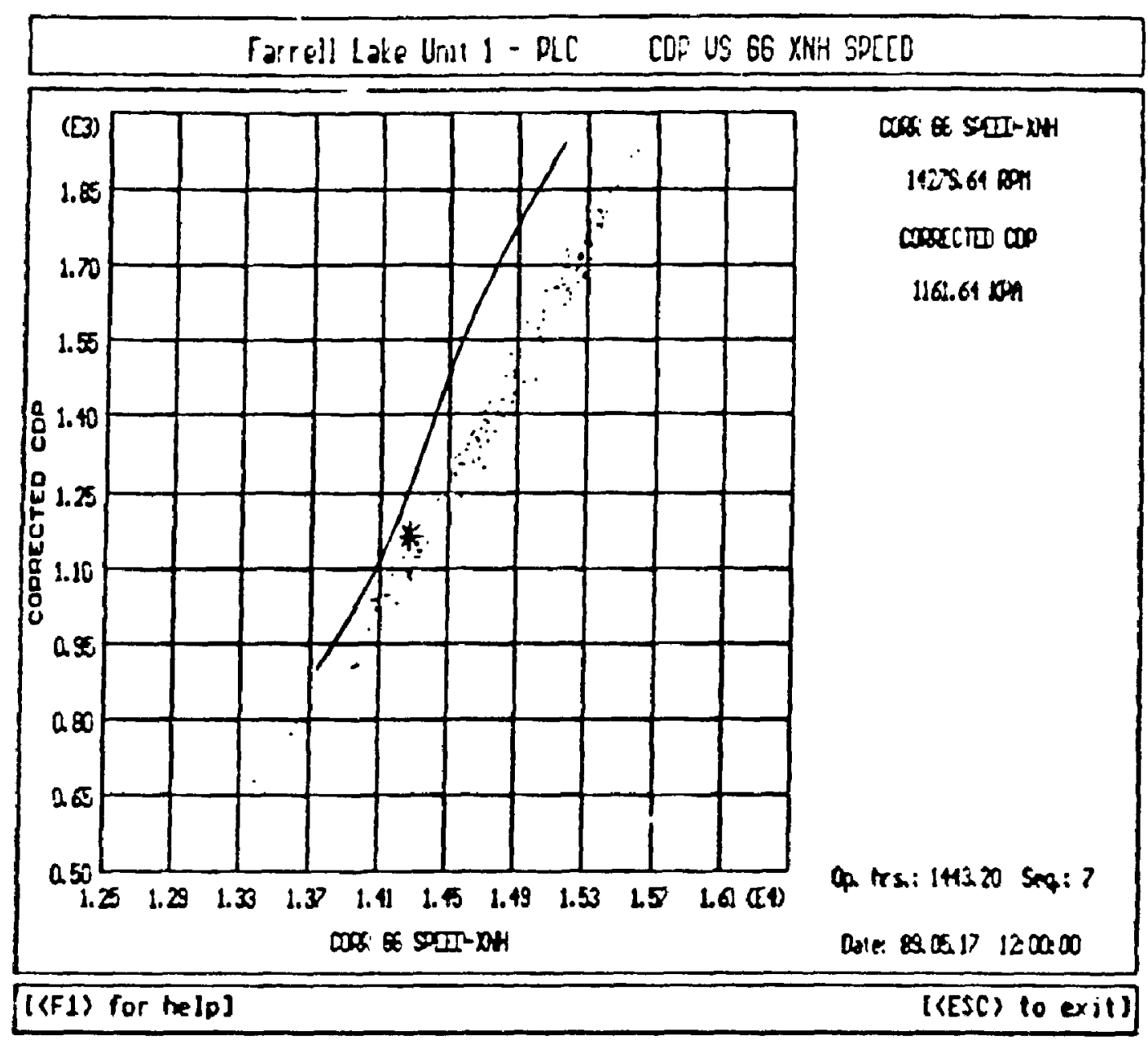

Figure 28. Comparison between Model Prediction and Field Measurement ( $P_{3 a t a s c}$ vs $N_{h}$ ) 


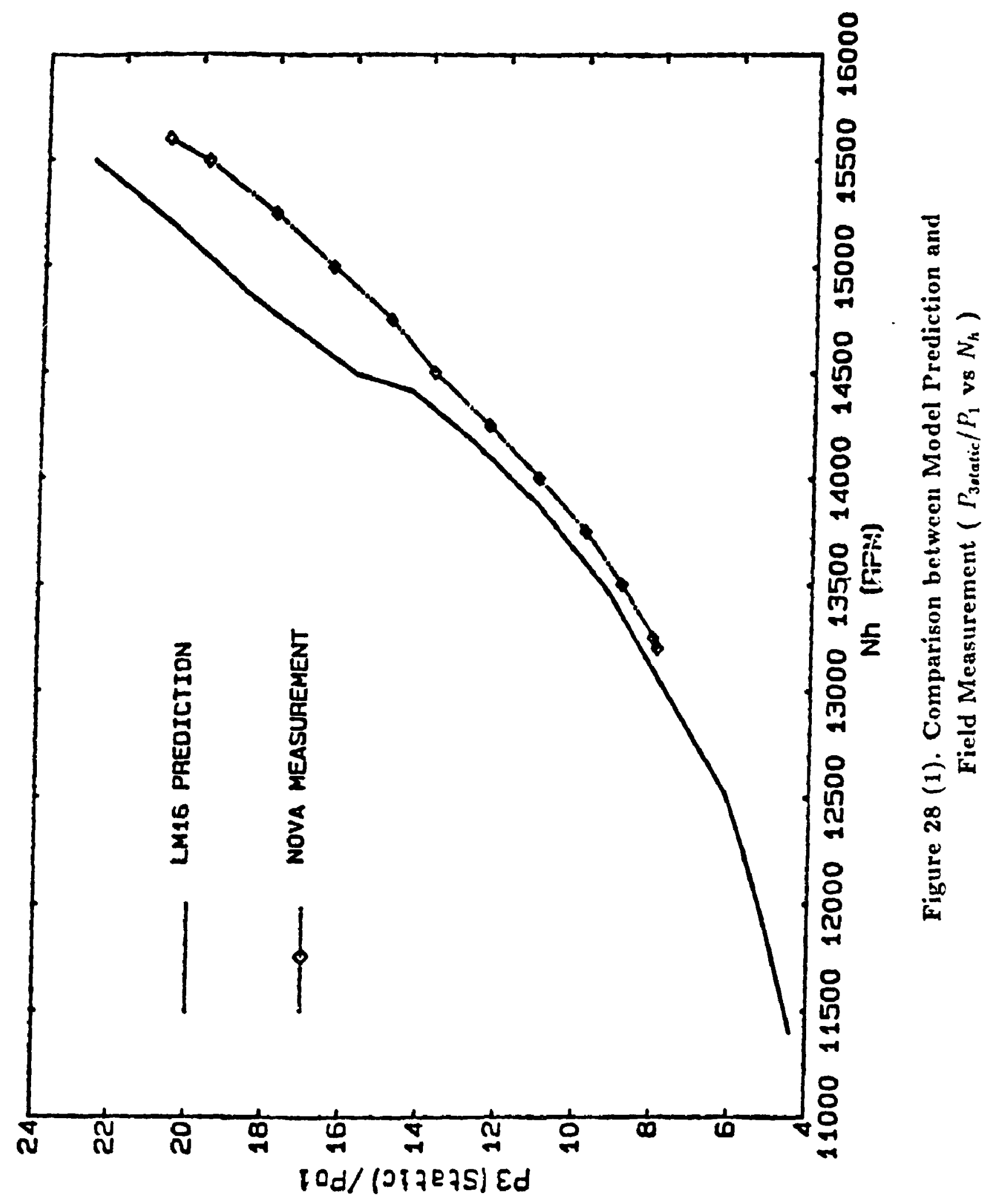




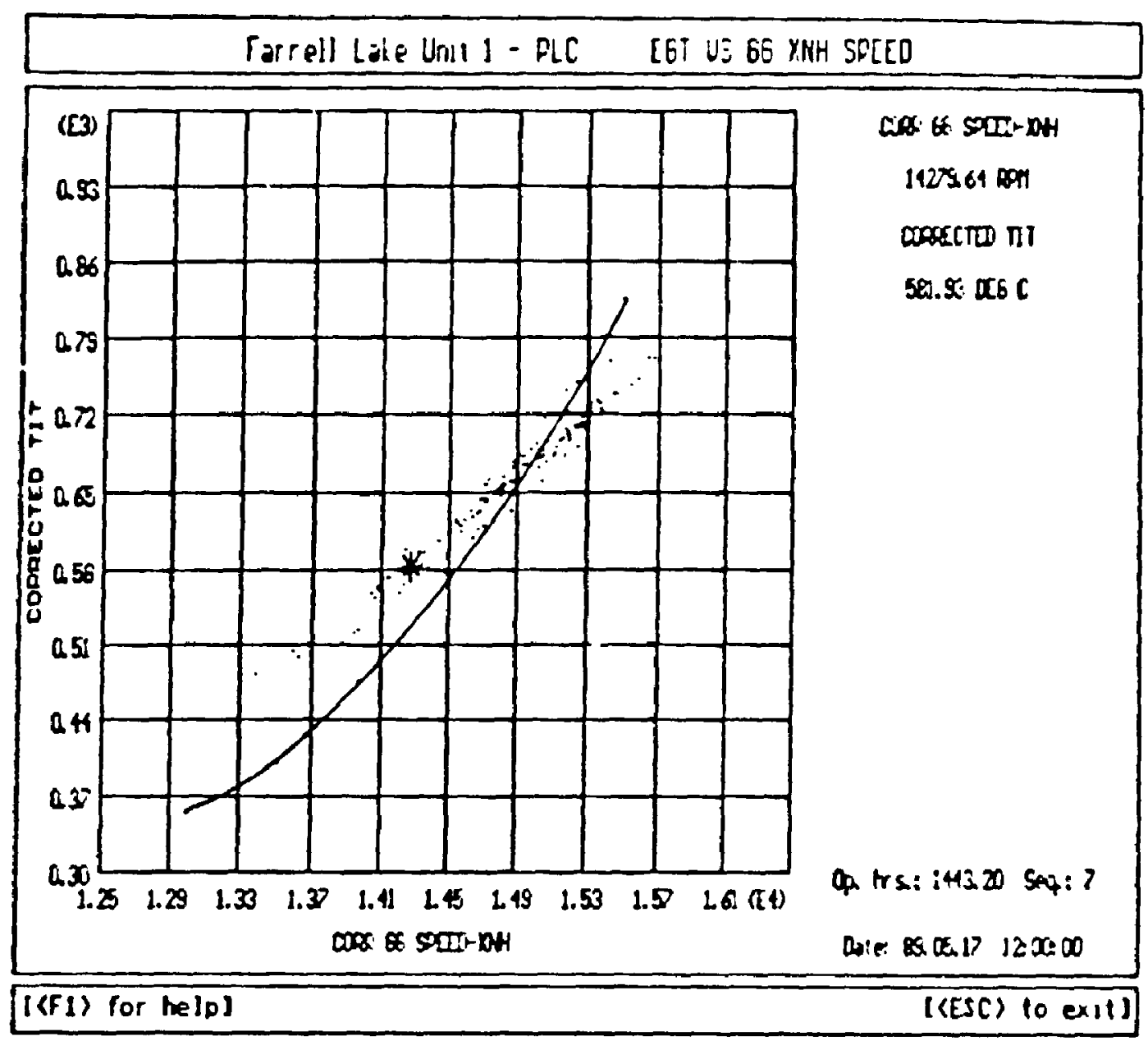

Figure 29. Comparison between Model Prediction and Field Measurement ( EGT vs $N_{h}$ ) 


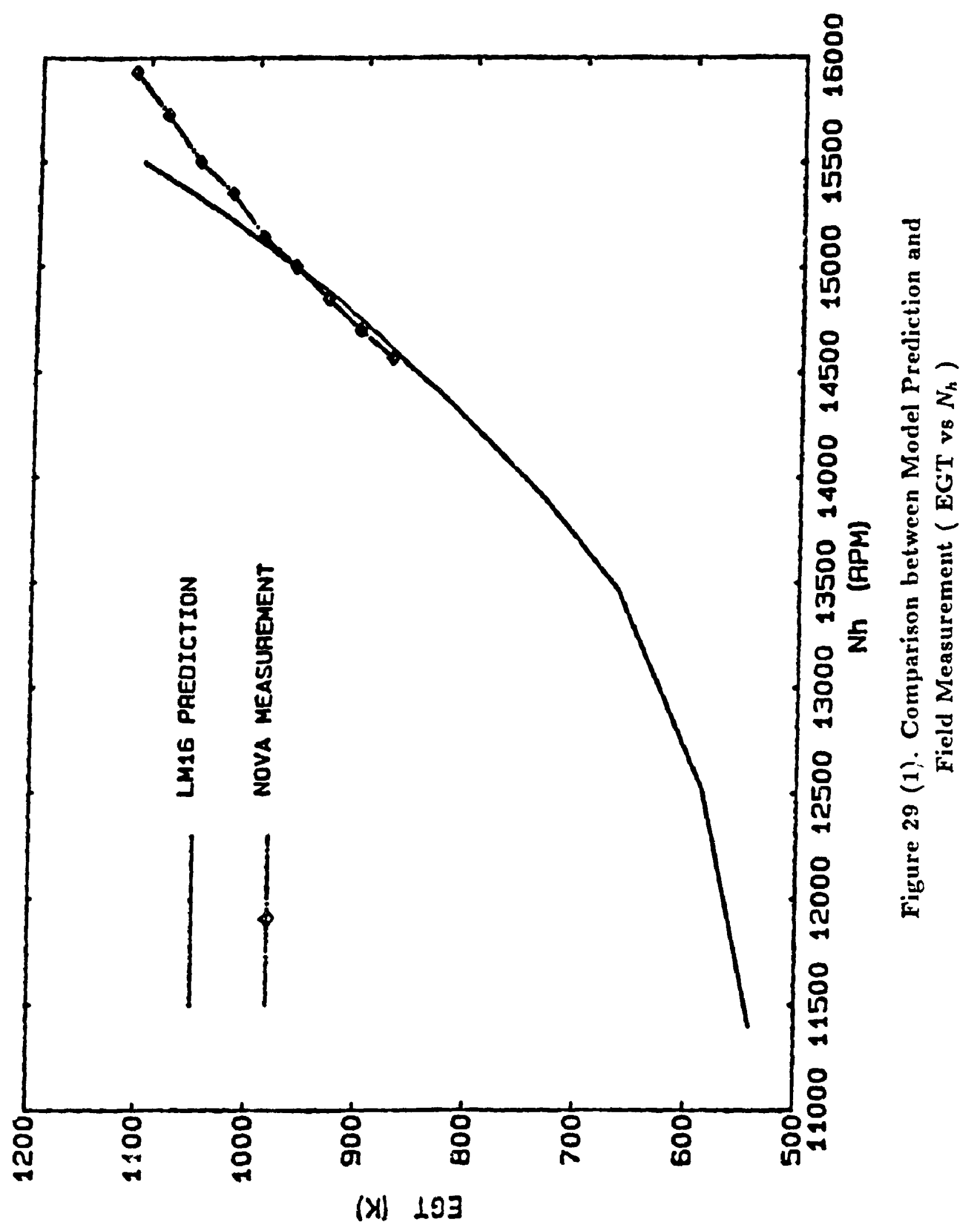




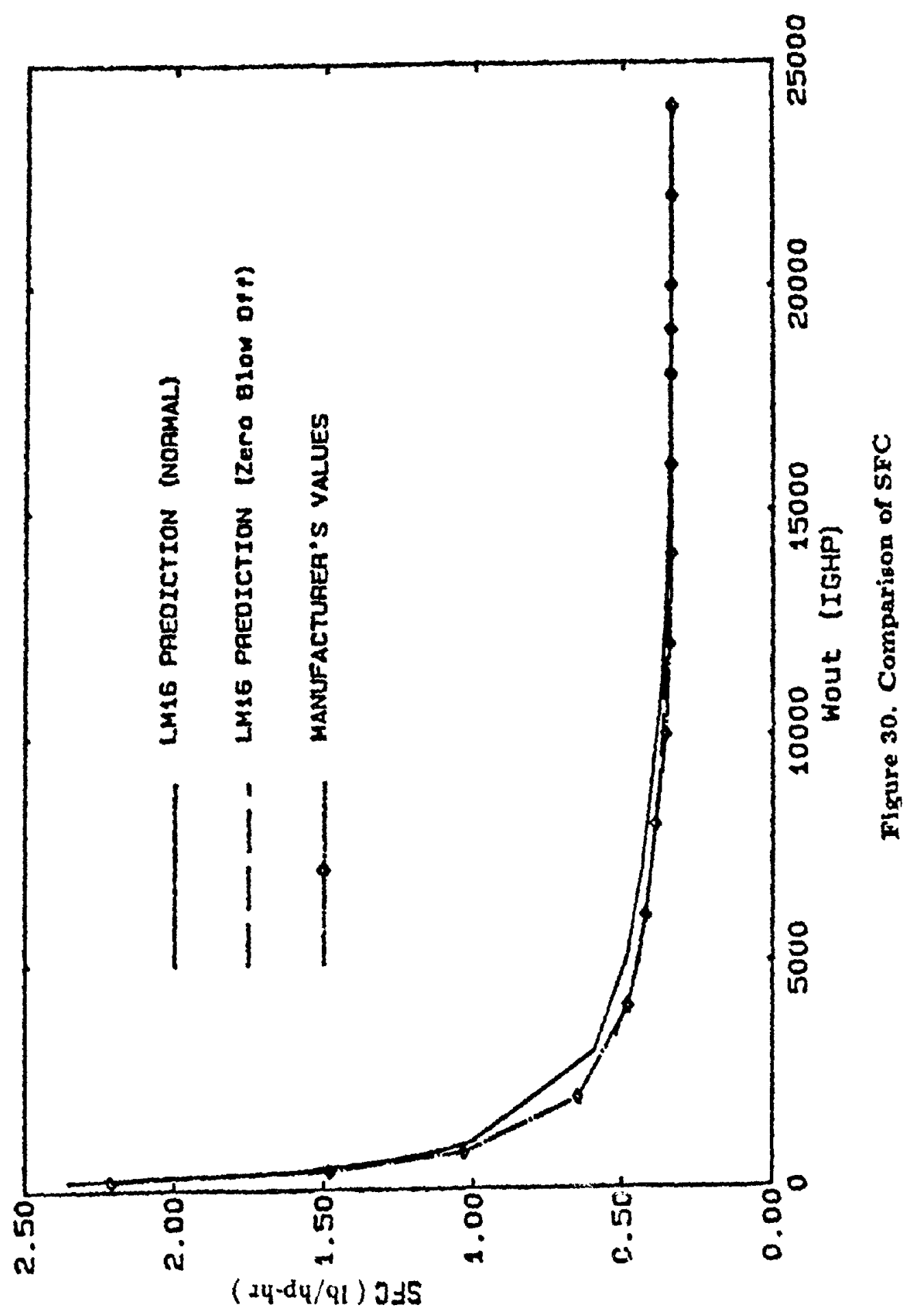




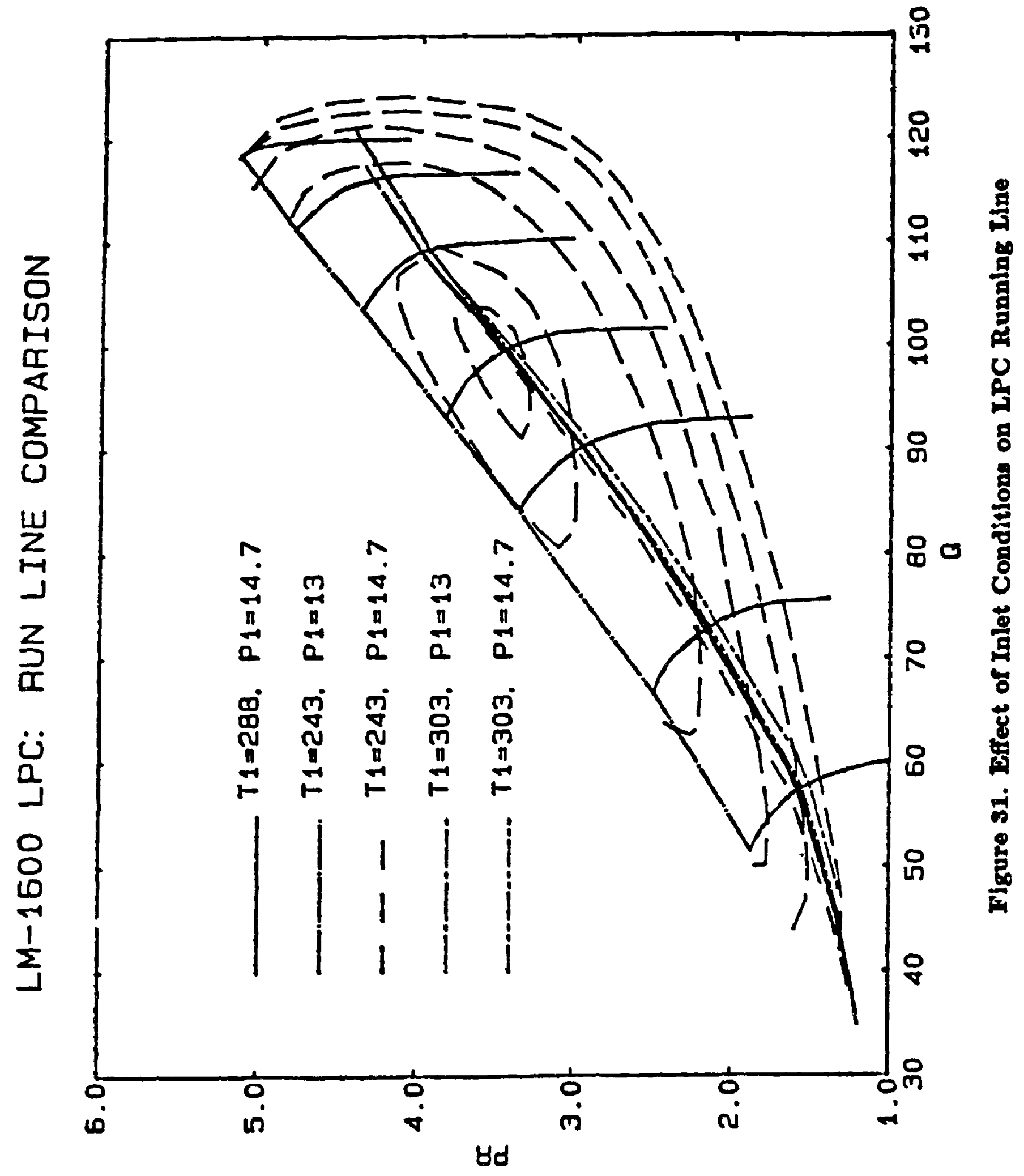




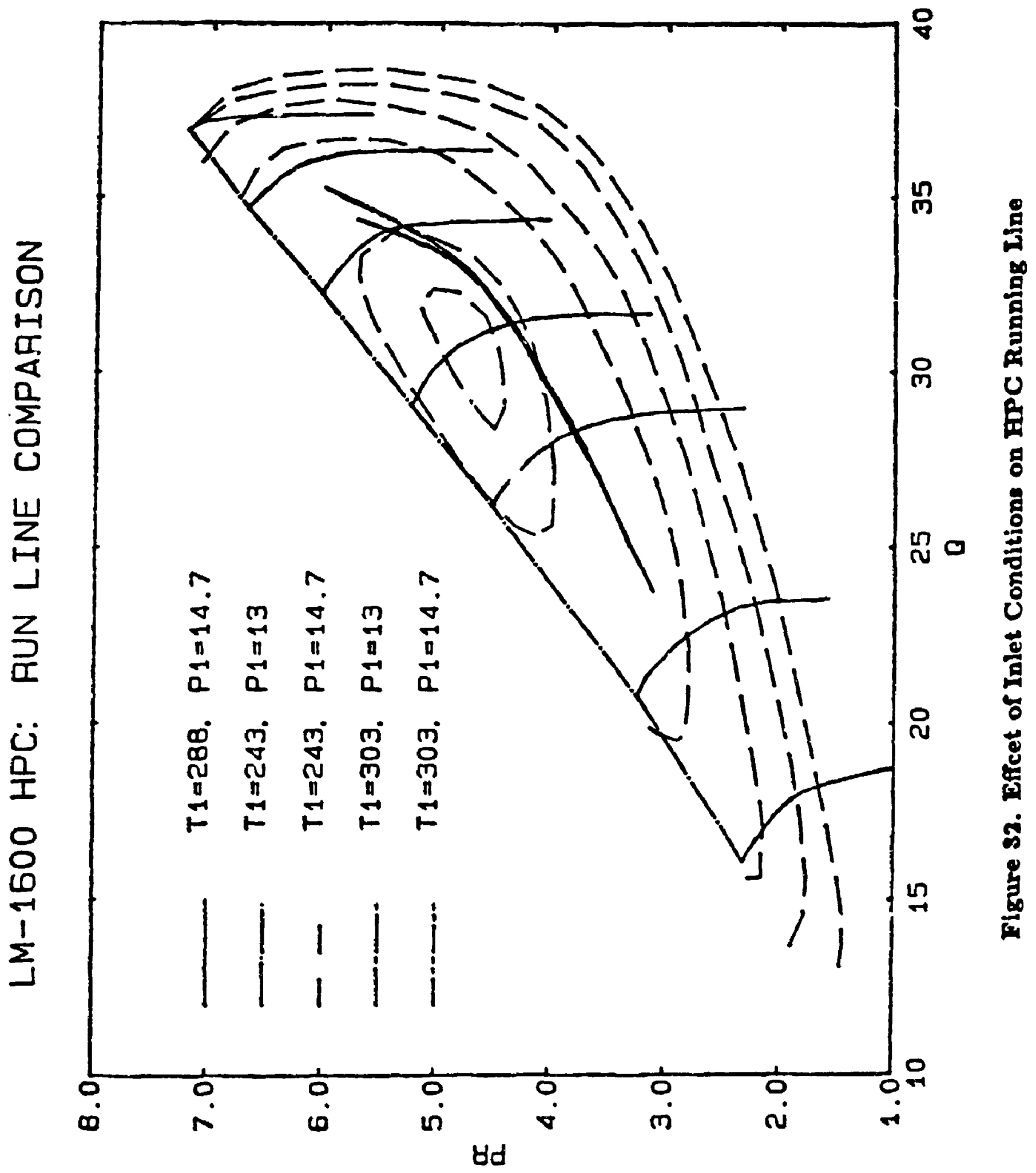


Deterforation 1: OPR VS Wout

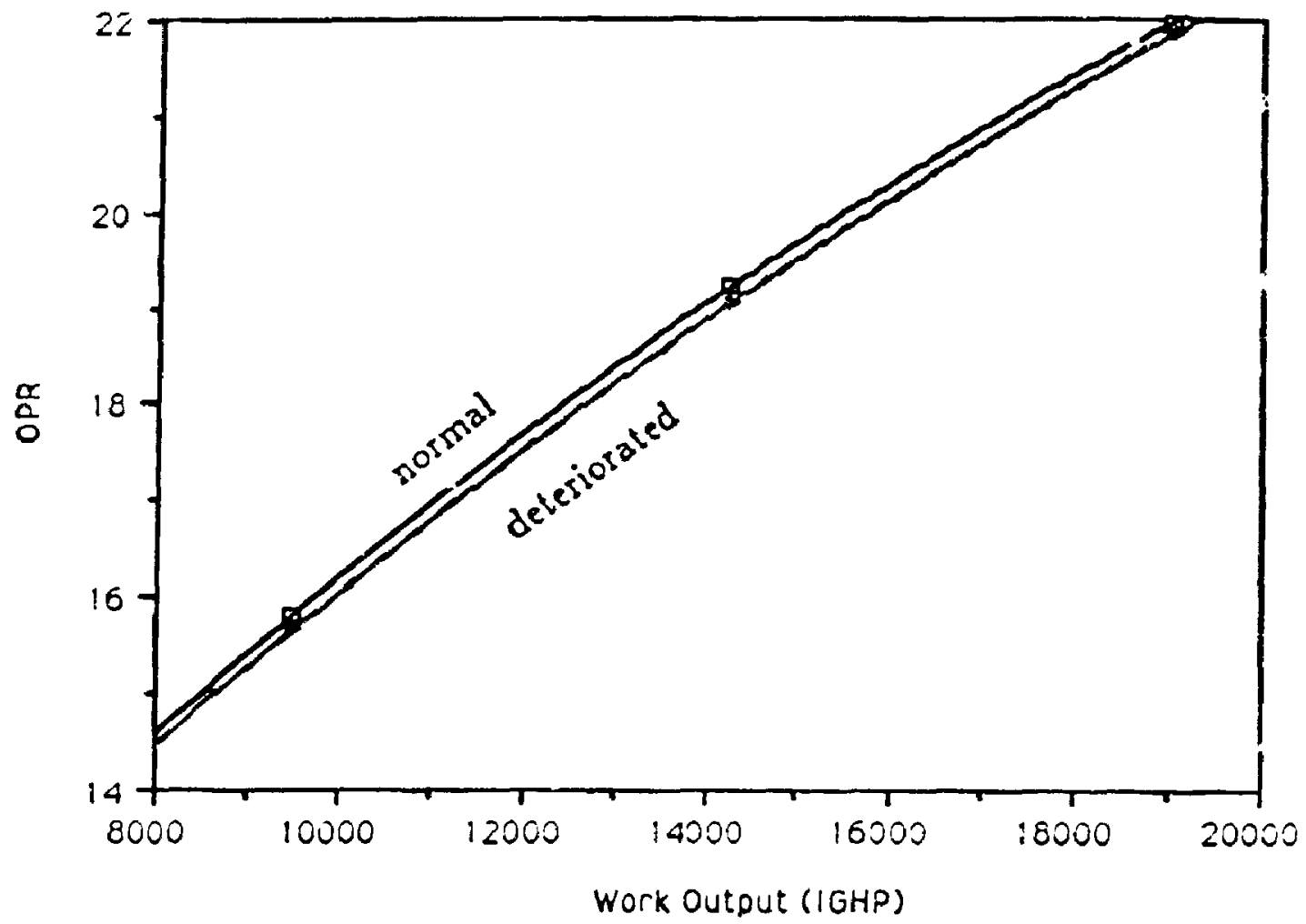

Deterloraition 1: TIT vi Wout

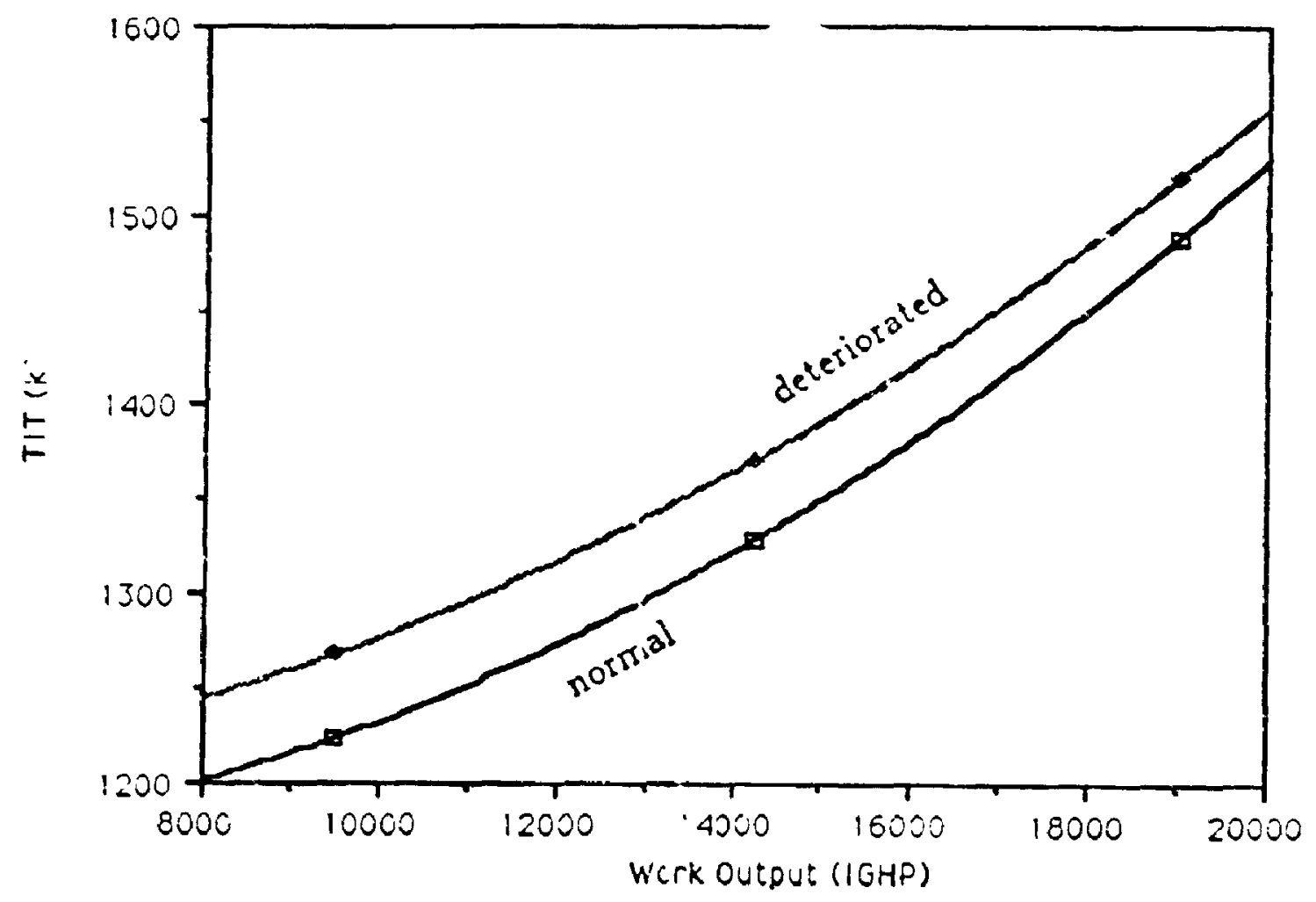

Fig. 33--1: LPC Mechanical Damage 
Deterioration 1: Fuel Flow vs Wout

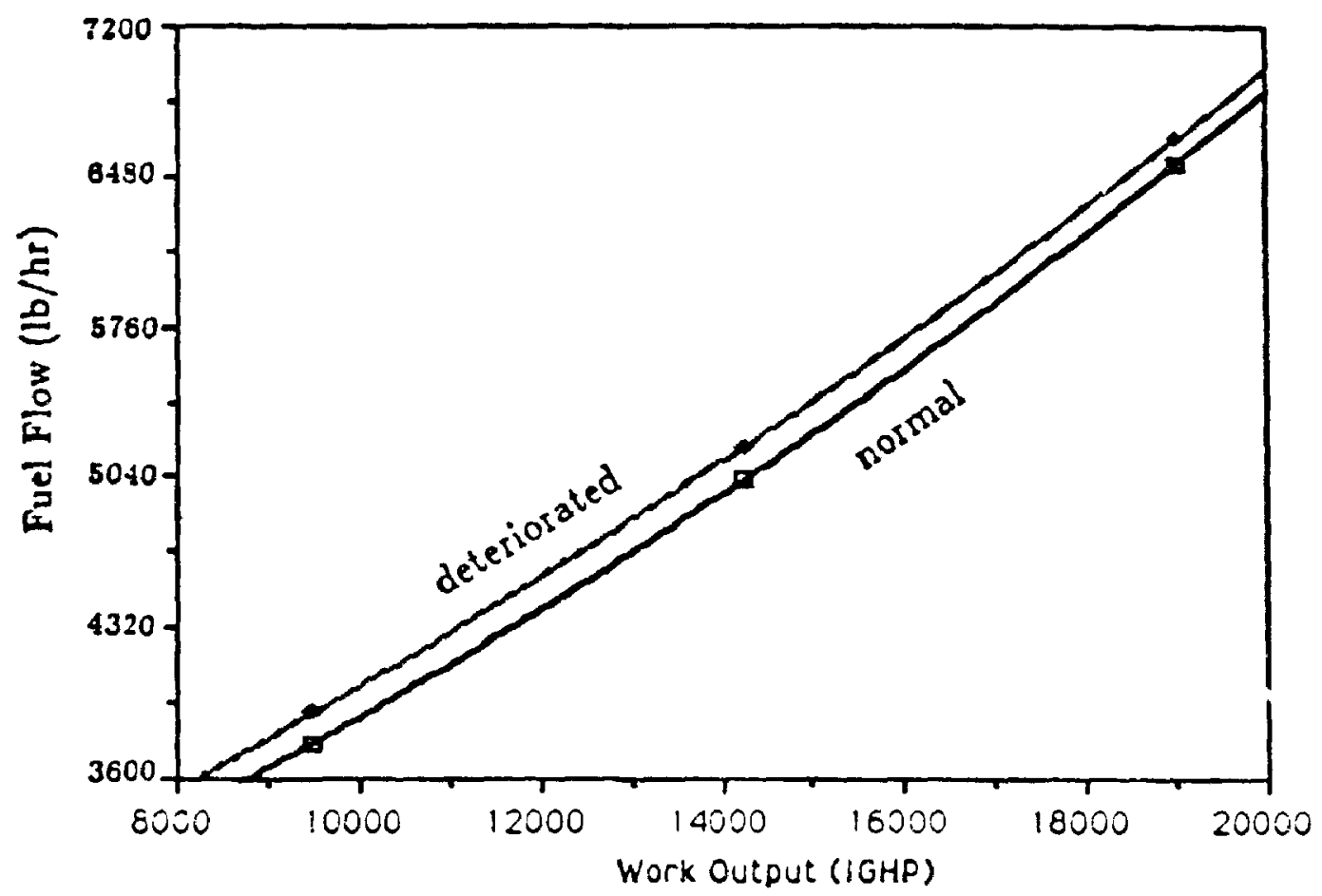

Deterioration 1: LP-Speed vs Wout

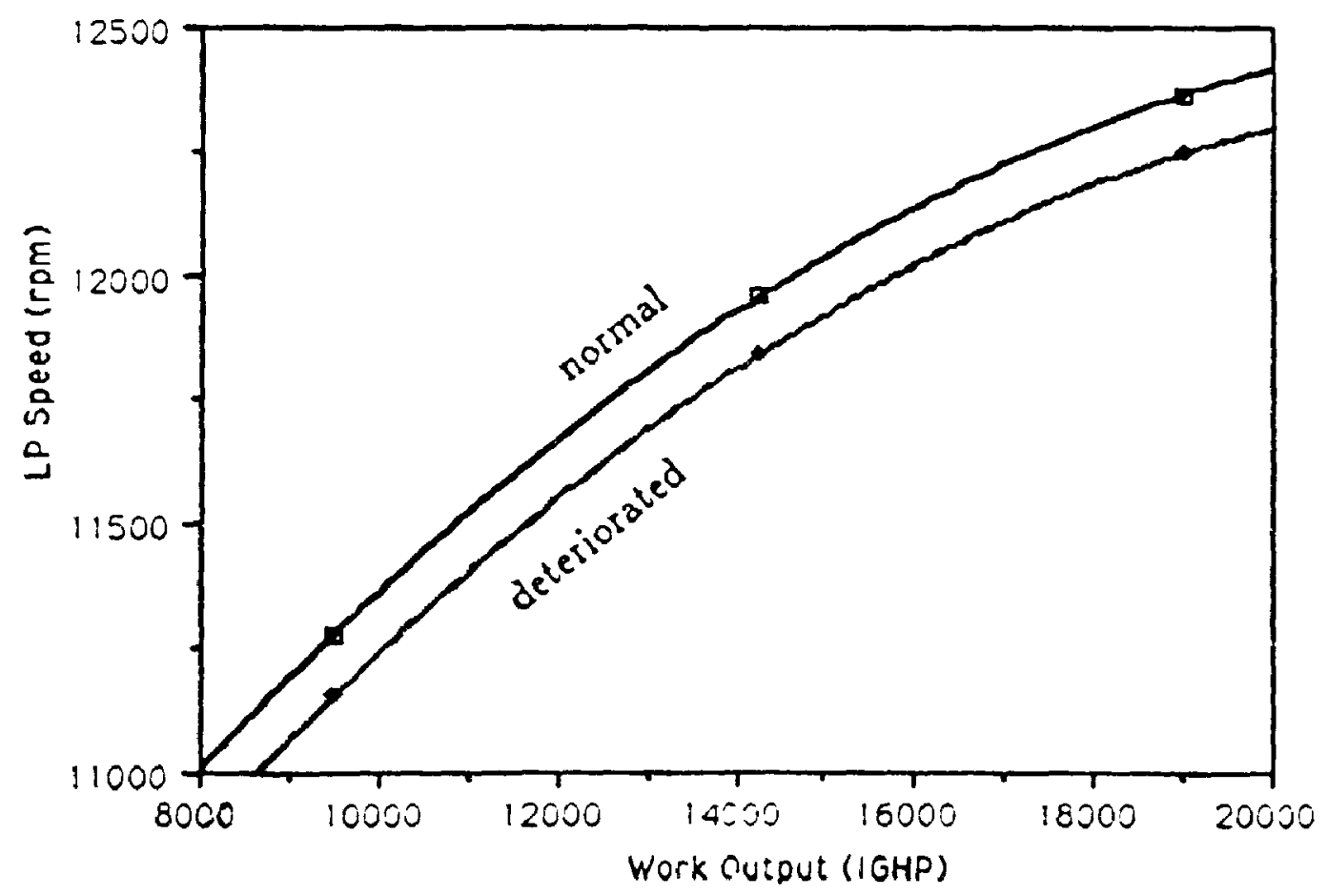

Fig 33--2. LPC Mechanical Damage 
Deterioration 2. OPR vs Wout

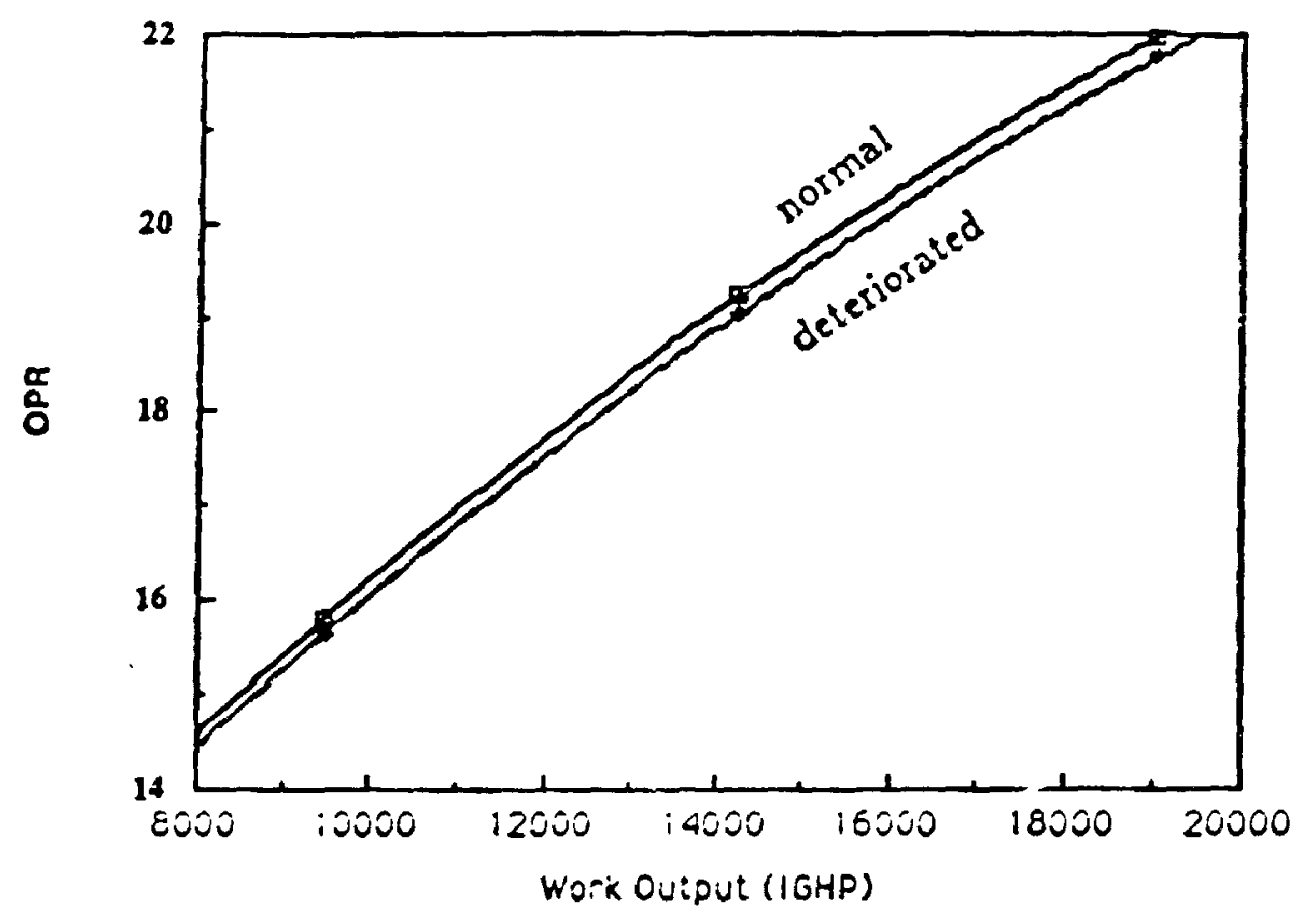

Deterloration 2 : TIT Vs Wout

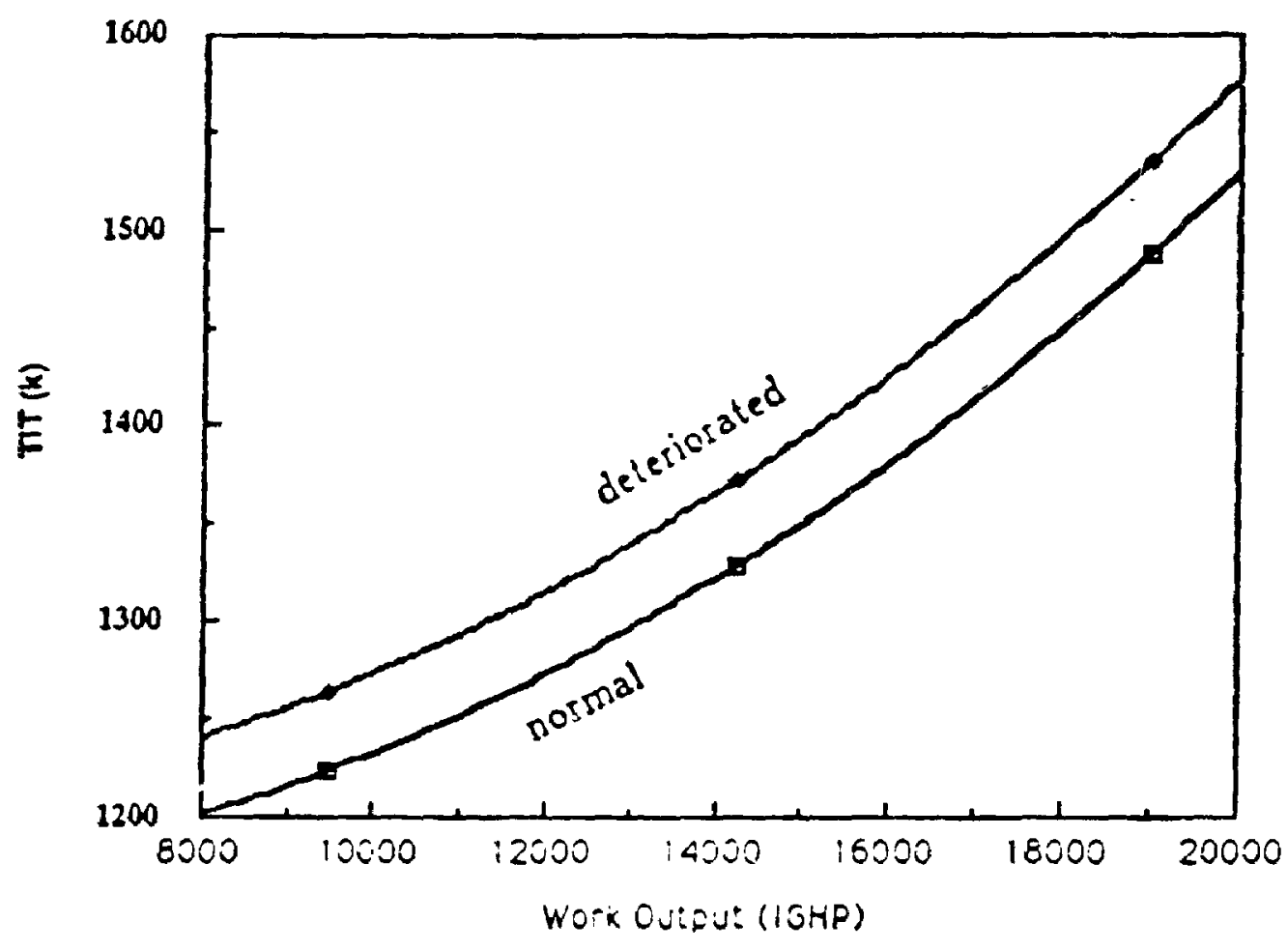

Fig :-:-- I. Compressor Excessive Leakage 
Deterioration 2: Fuel Flow vs Wout

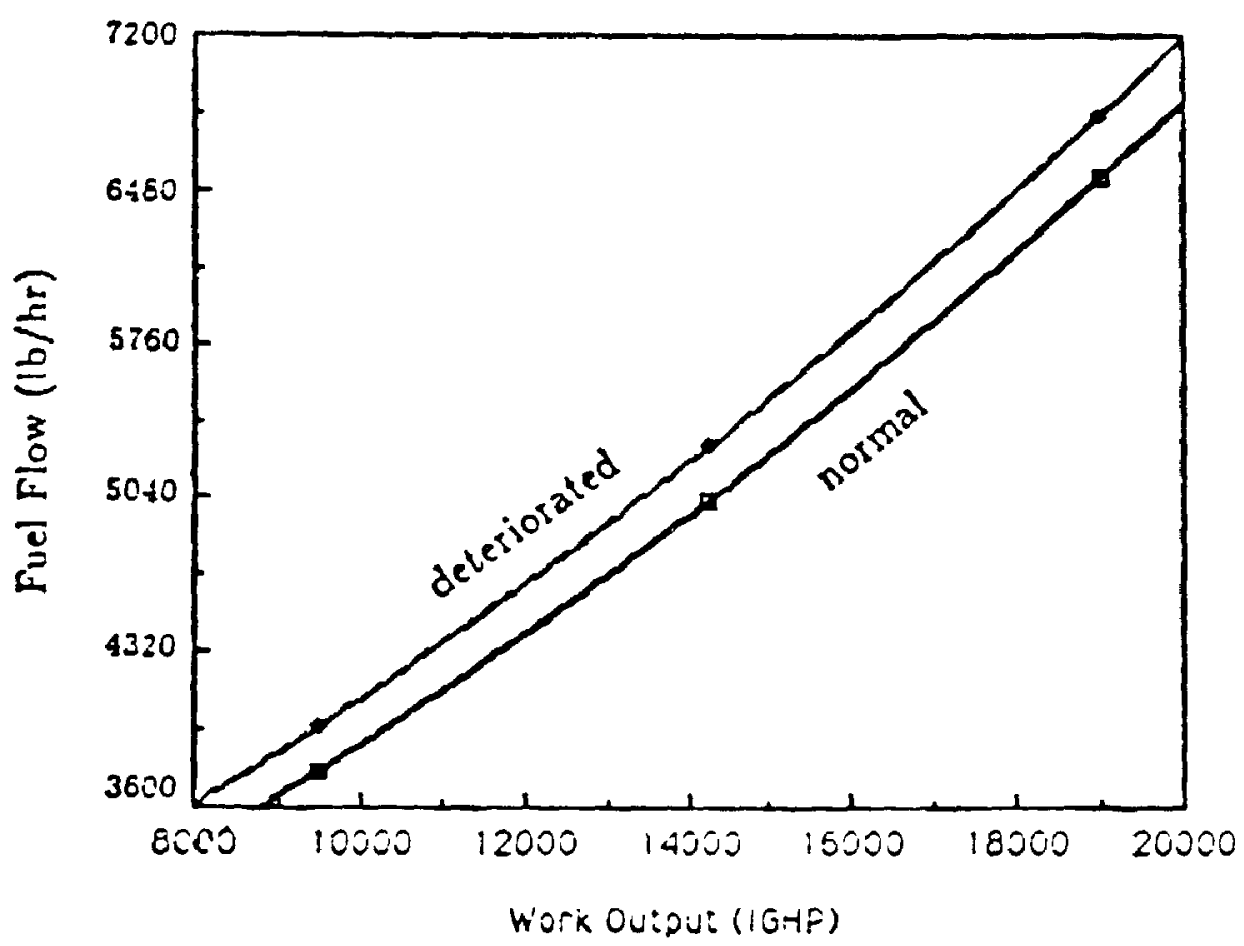

Dtereioration 2 : LP-Speed vs Wout

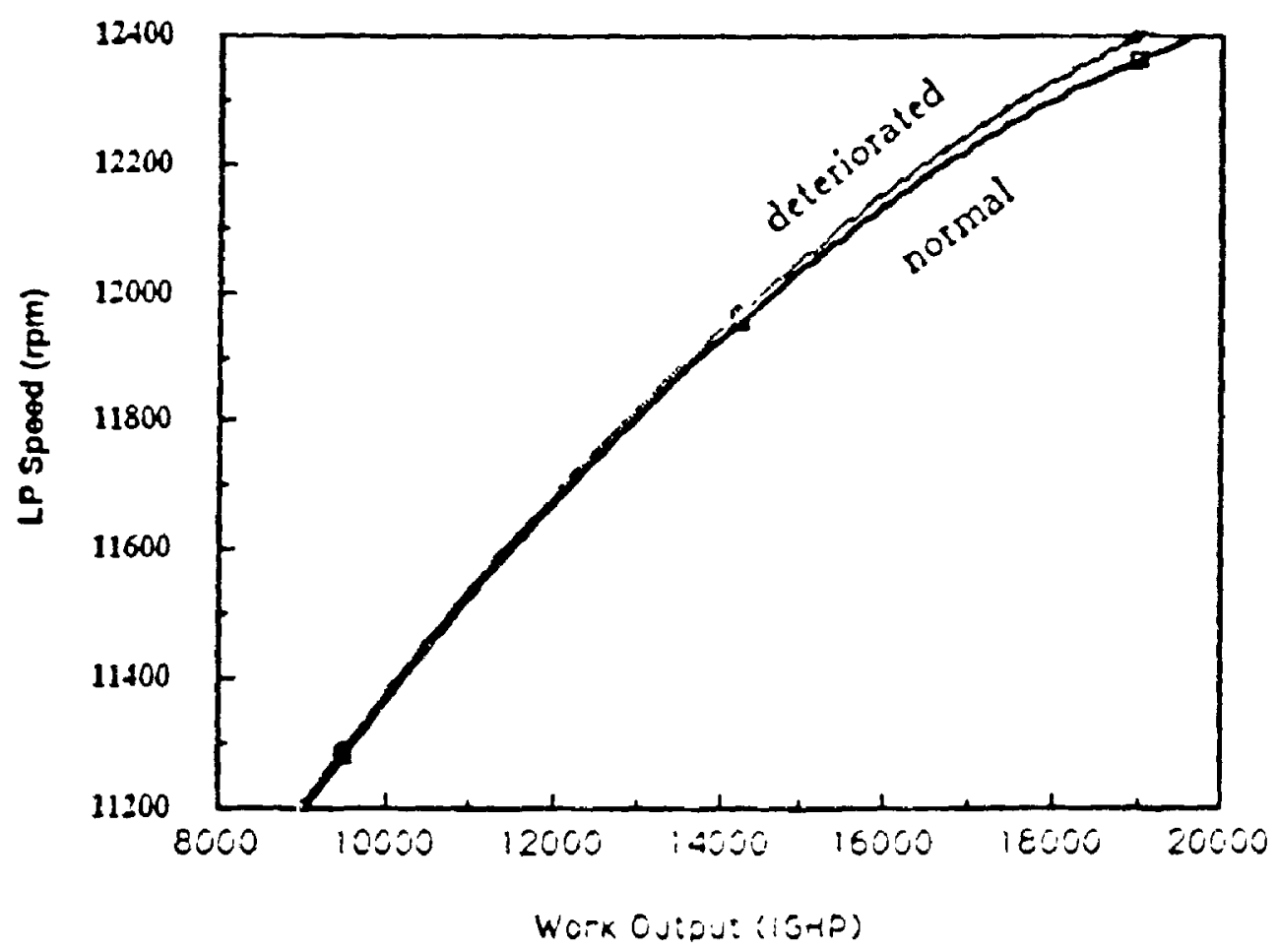

Fig 34--2 Compresscr Excesslve Lezkage 
Deterioration 3: OPR vs Wout

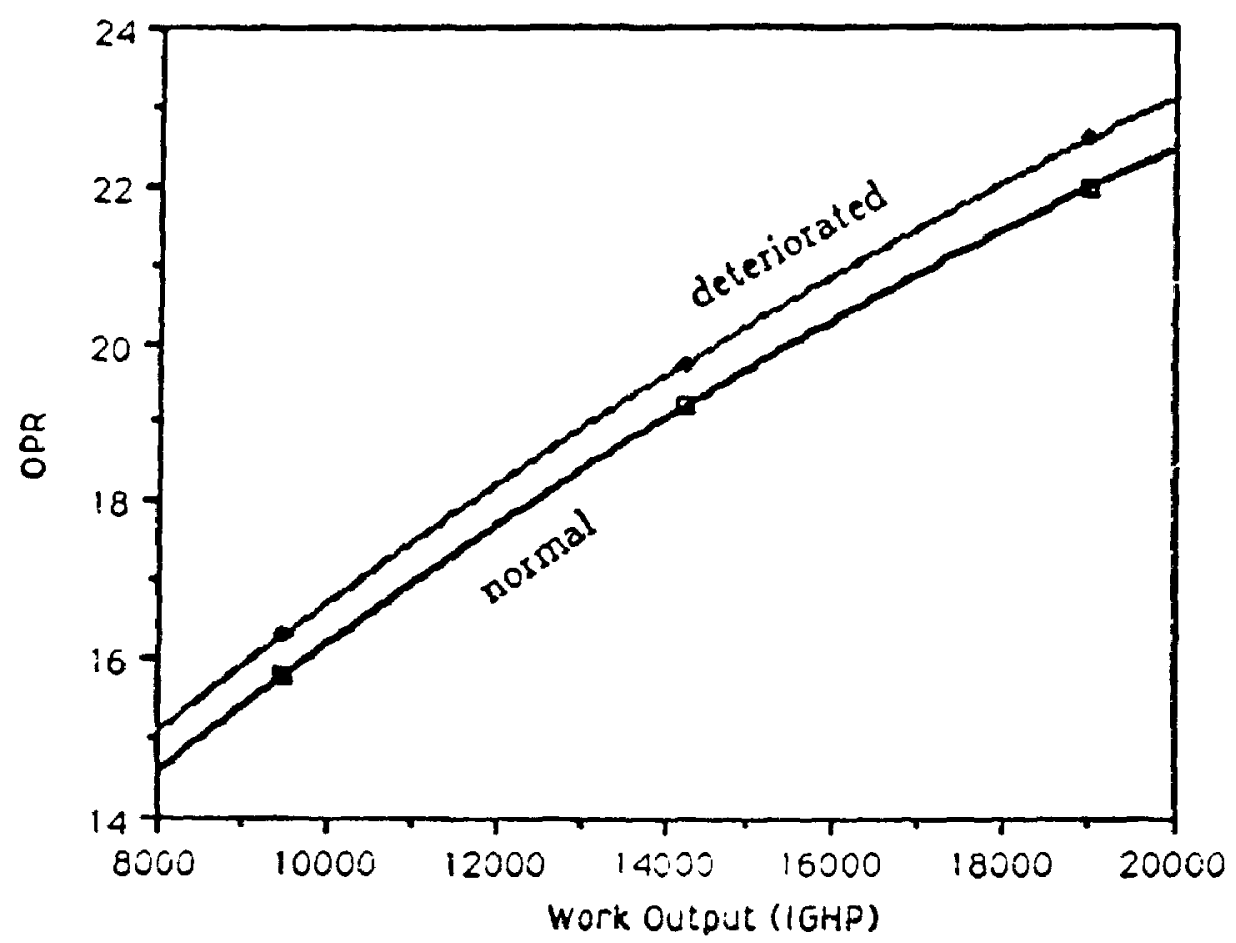

Deterioration 3: TIT vs Wout

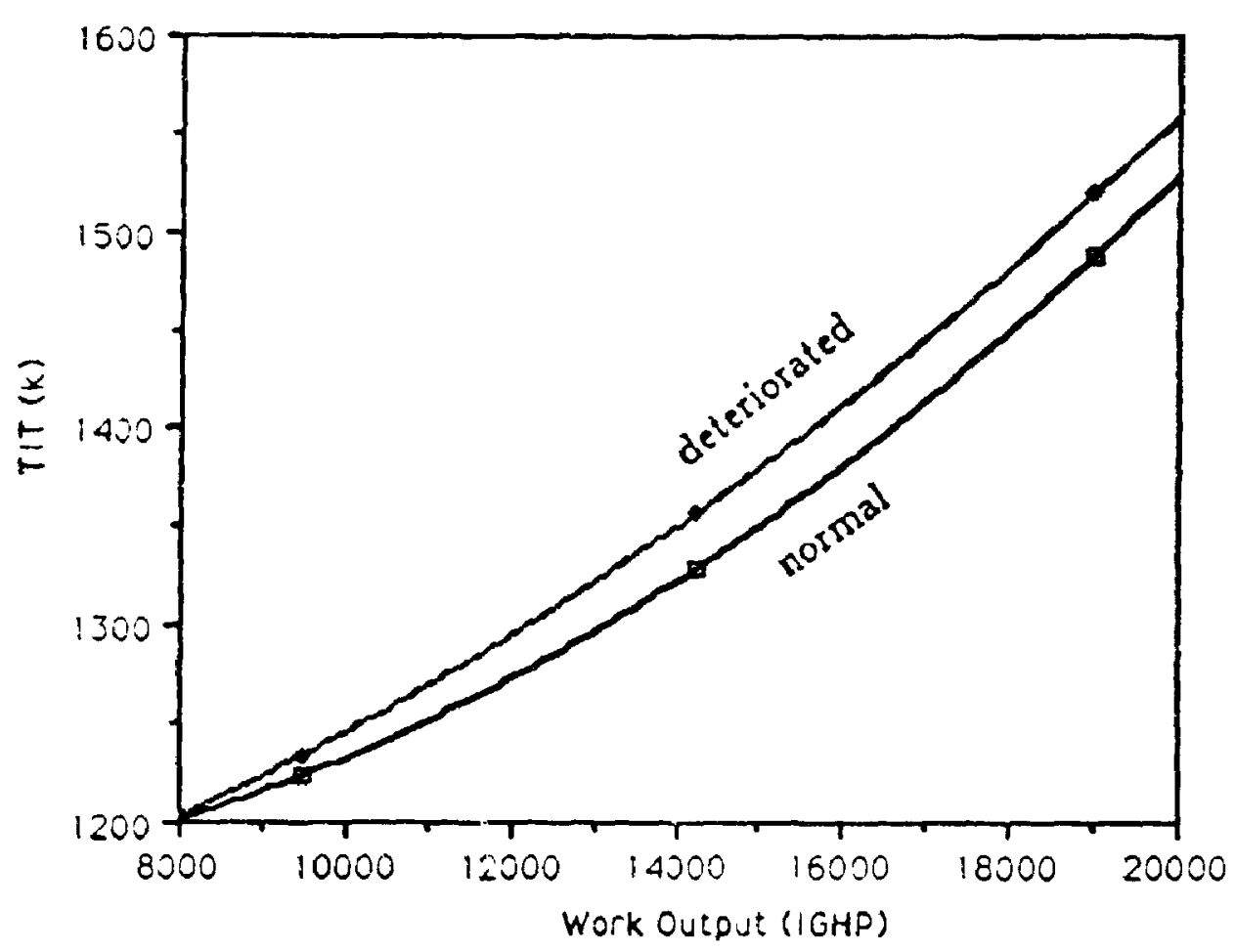

Fig ij--1. HPT Mechanicai Damage 
Deterioration 3: Fuel Flow vs Wout

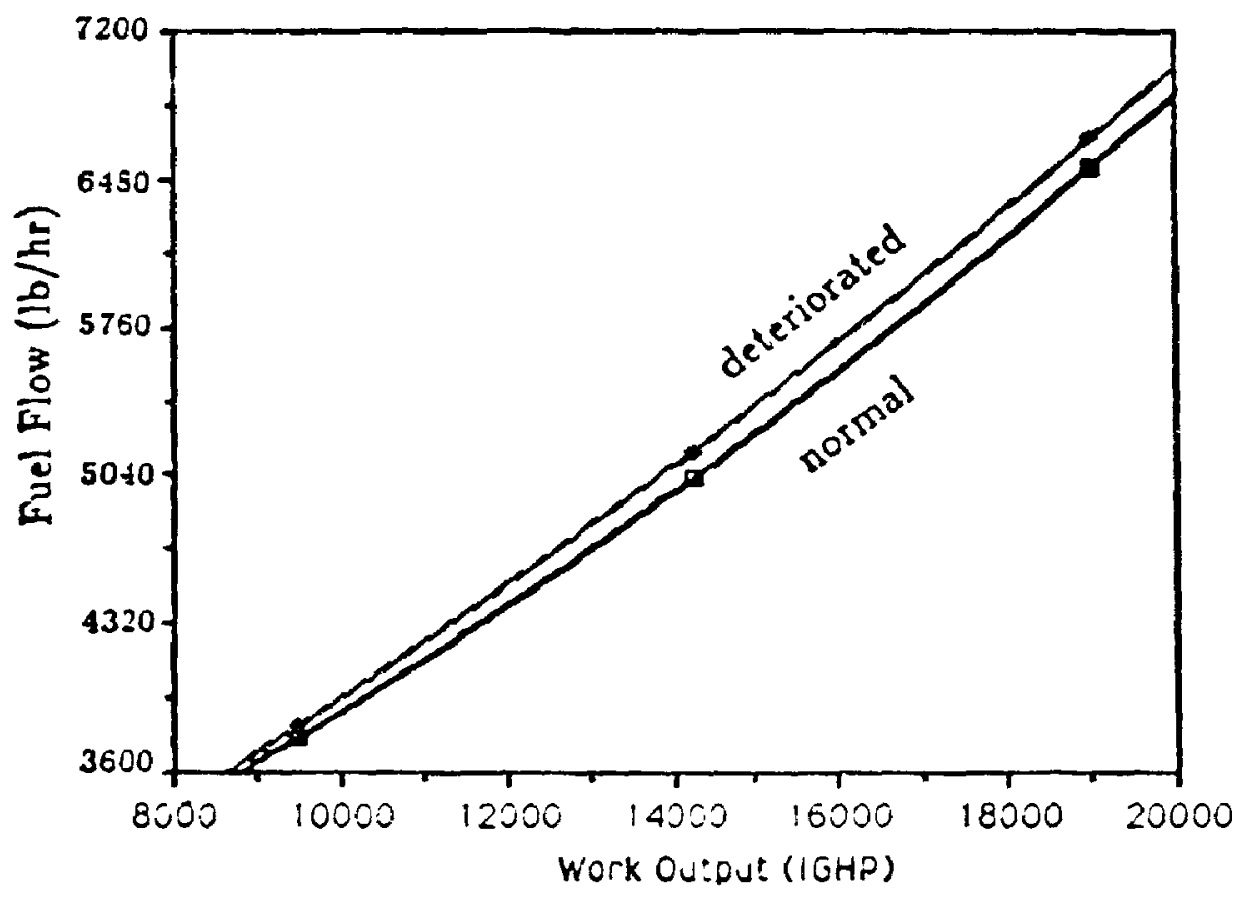

Deterioration 3: LP-Speed vs Wout

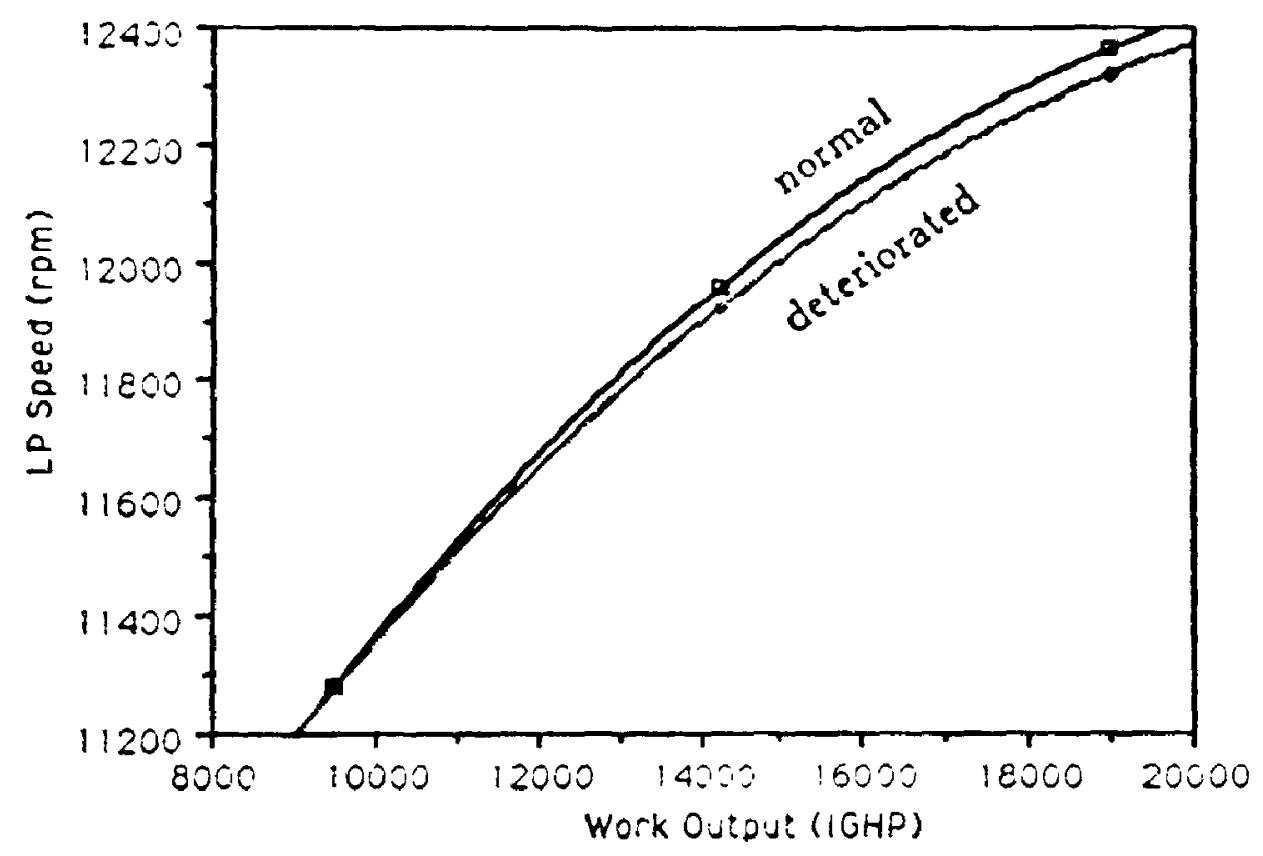

F!g 35--2 HPT Mechanical Damage 
Deterioration 4: OPR VS Wout

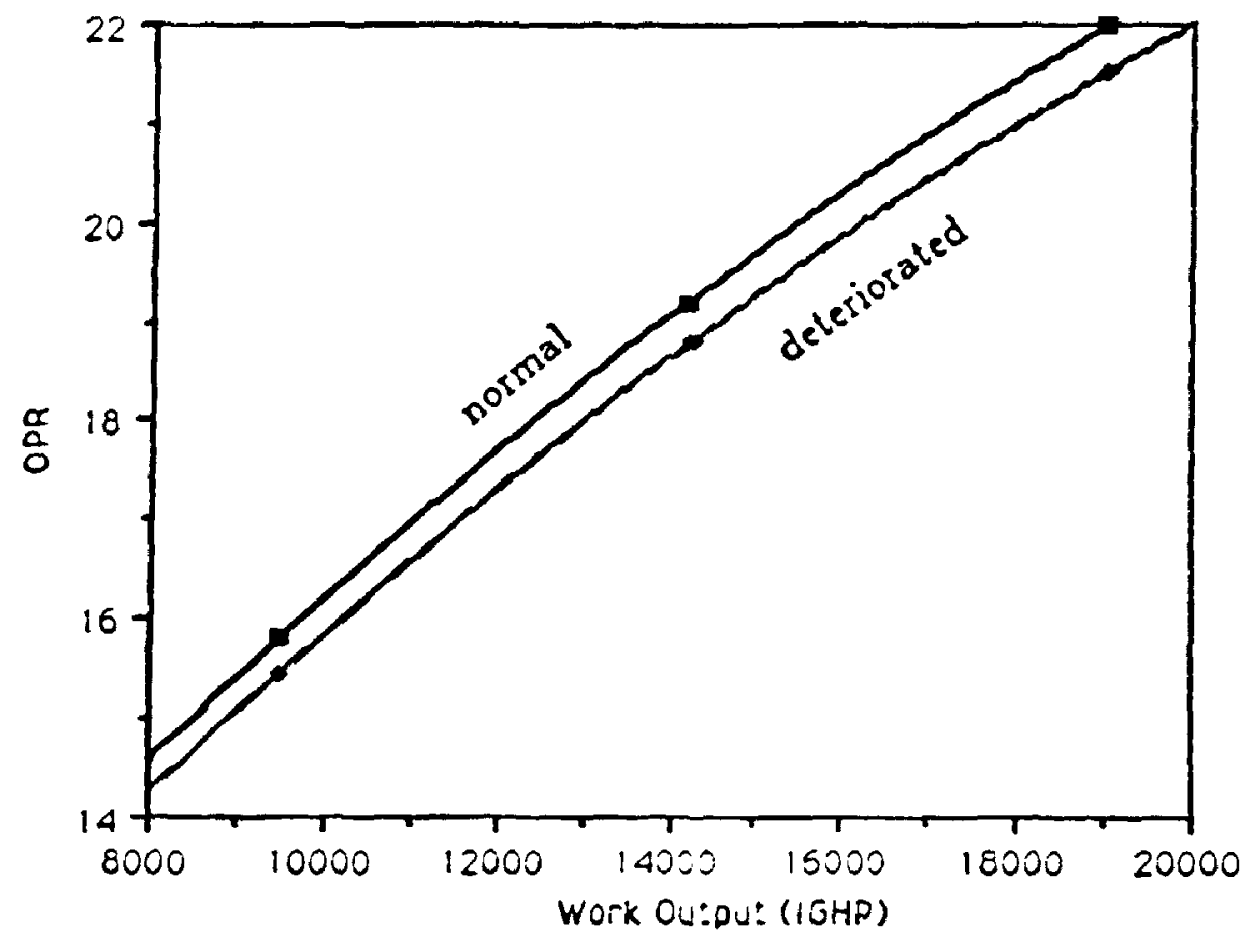

Deterioration 4: TIT VS Wout

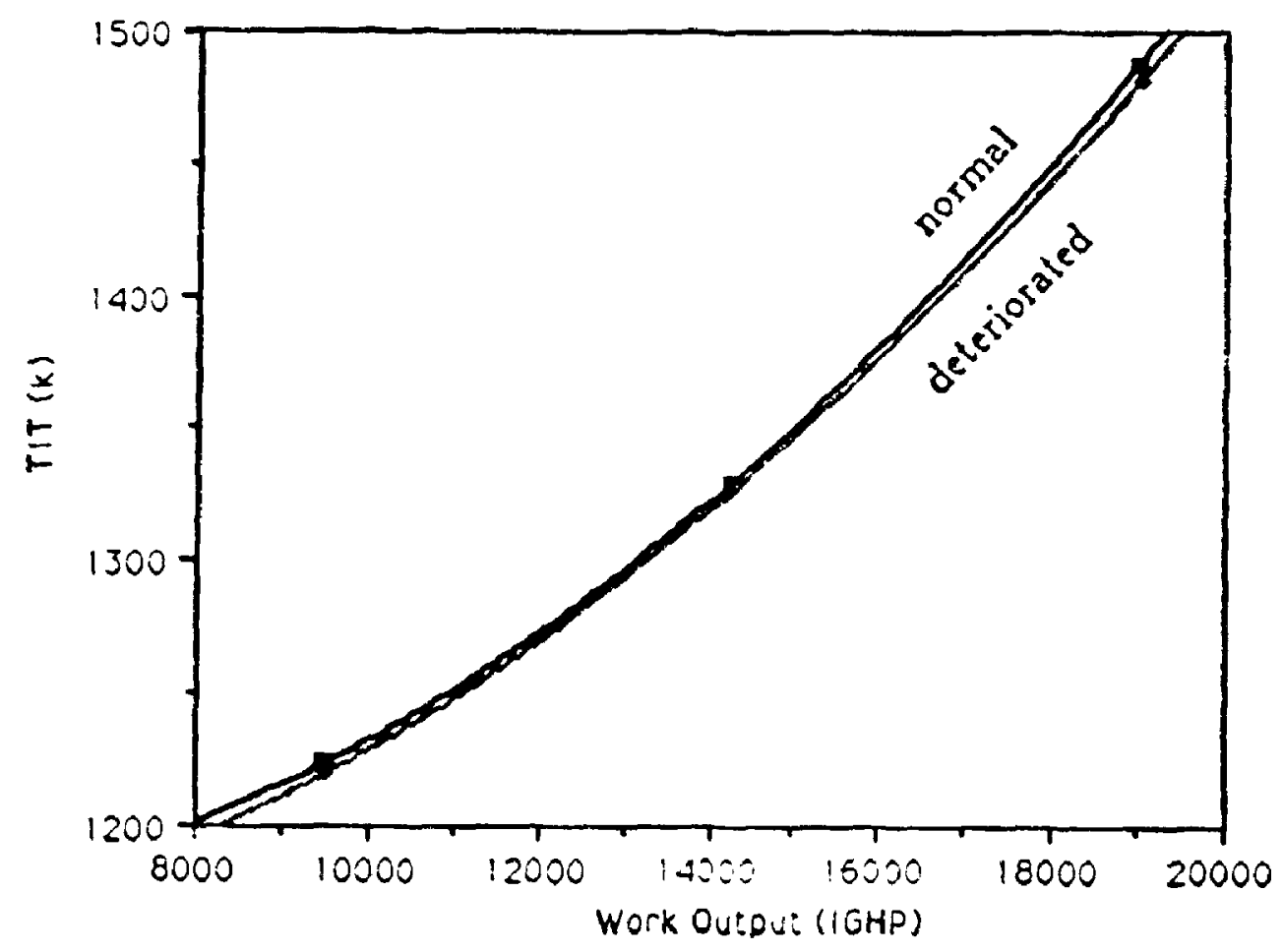

FIg 36--1. HPT Erosion 
Deterioration 4: Fuel Flow vs Wout

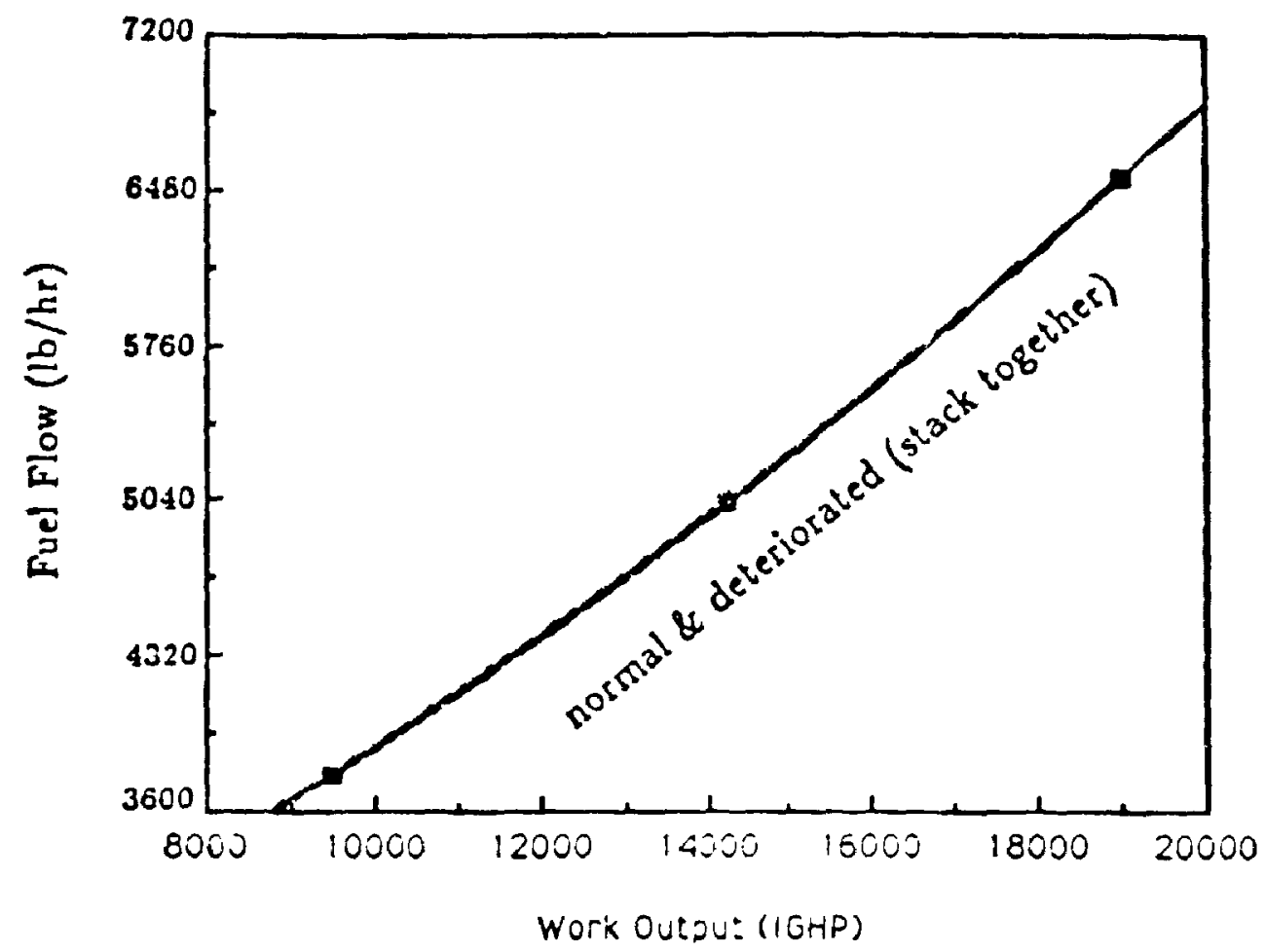

Deterioration 4. LP-Speed vs Wout

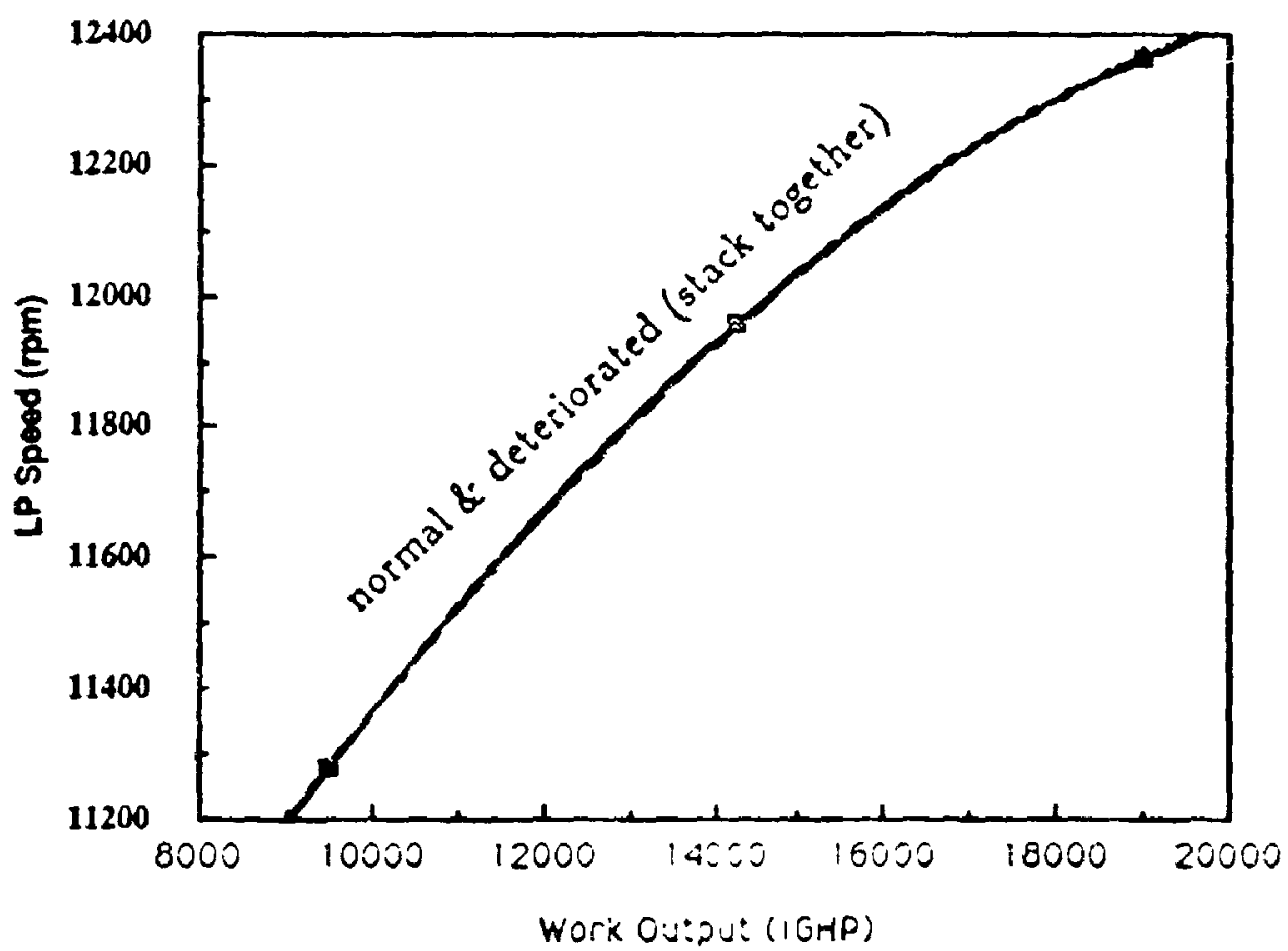

FIg 36--2 HiPT EROSION 
Deterloration 5: OPR vs Wout

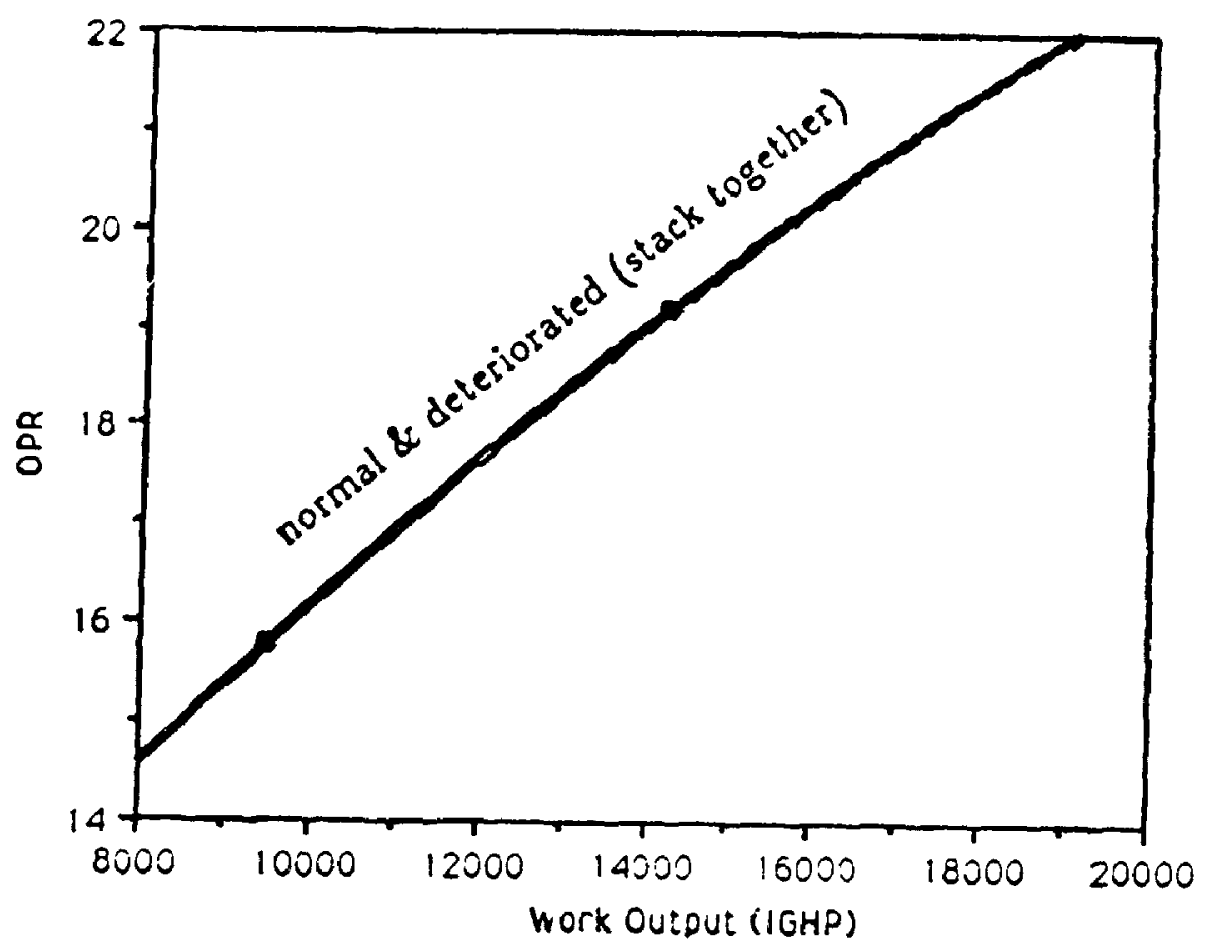

Deterioration 5. TIT vs Wout

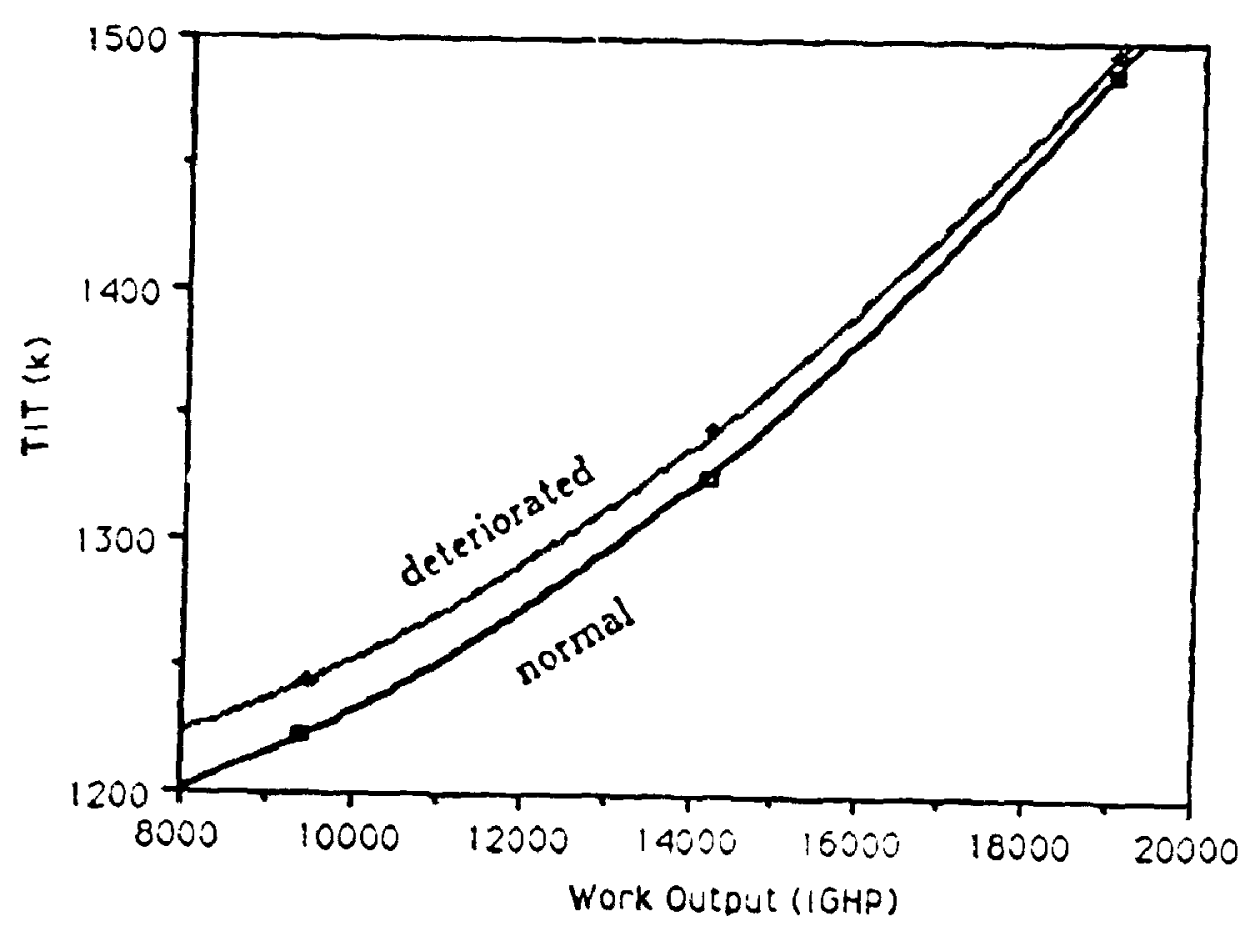

Fig. 37--1: LPT Erosion 
Deterioration 5: Fuel Flow vs Wout

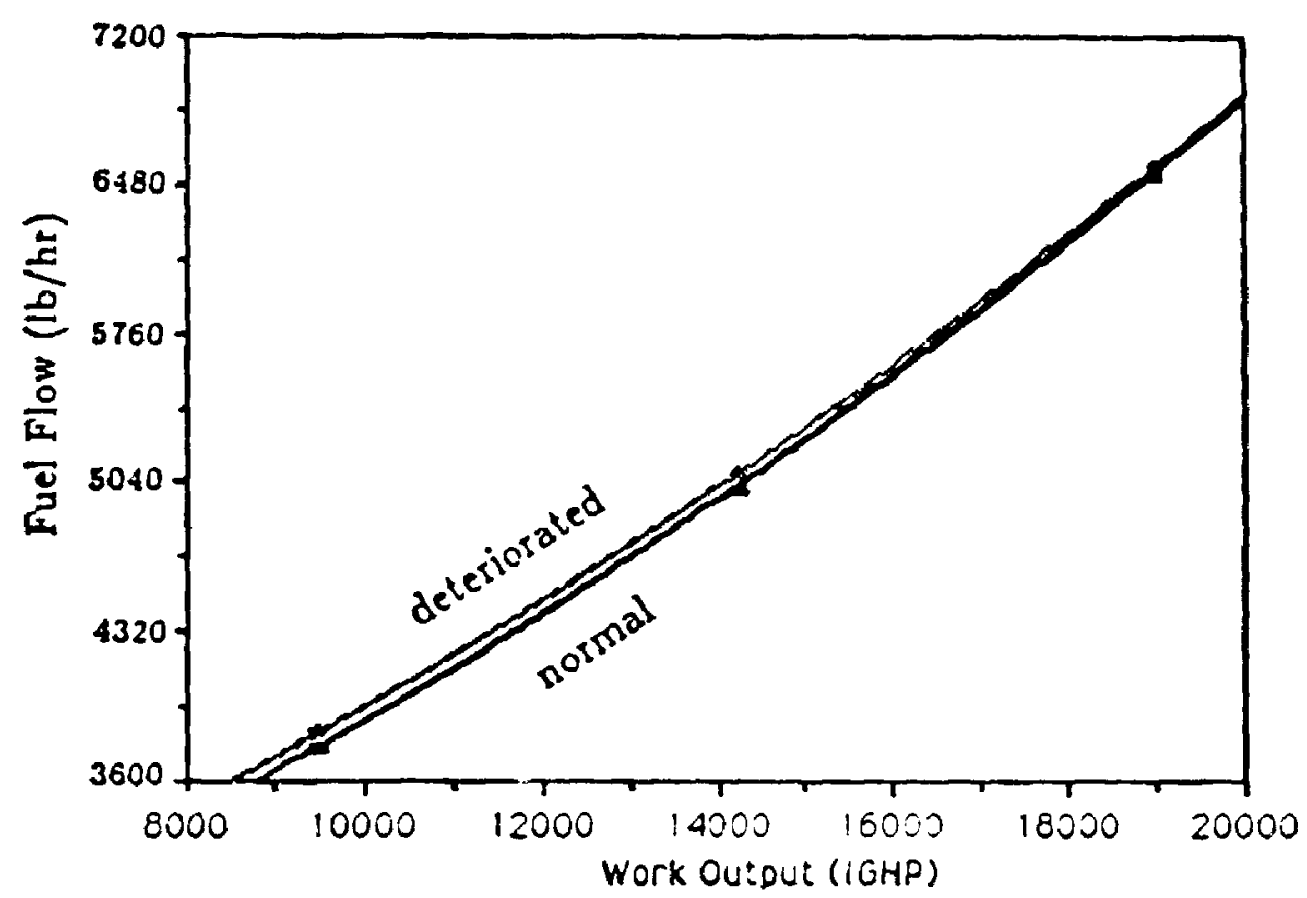

Deterioration 5. LP-Speed vs Wout

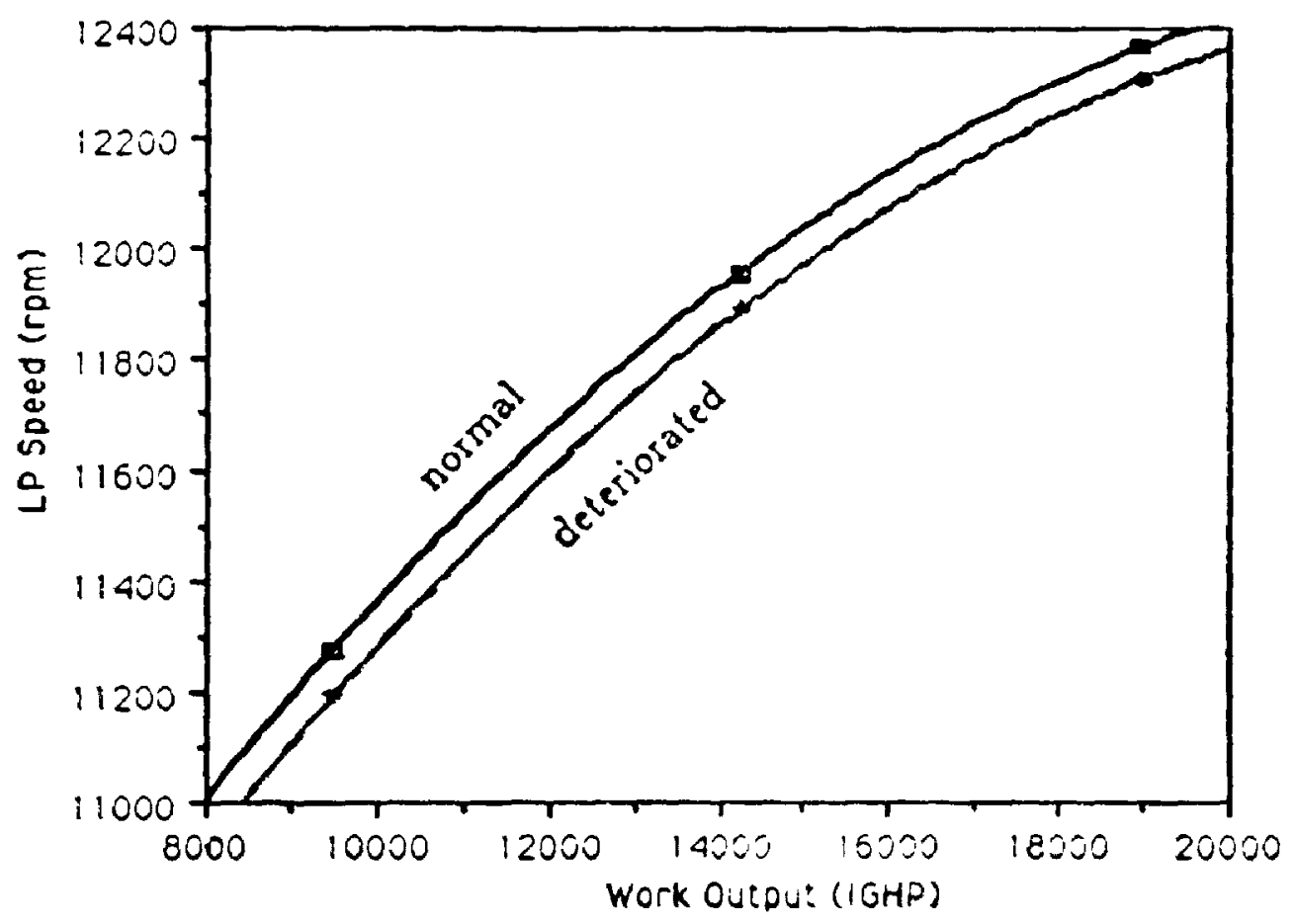

FIg. 37--2 LPT EROSIOn

156 
Deterloration 1: OPR VS LP-Speed

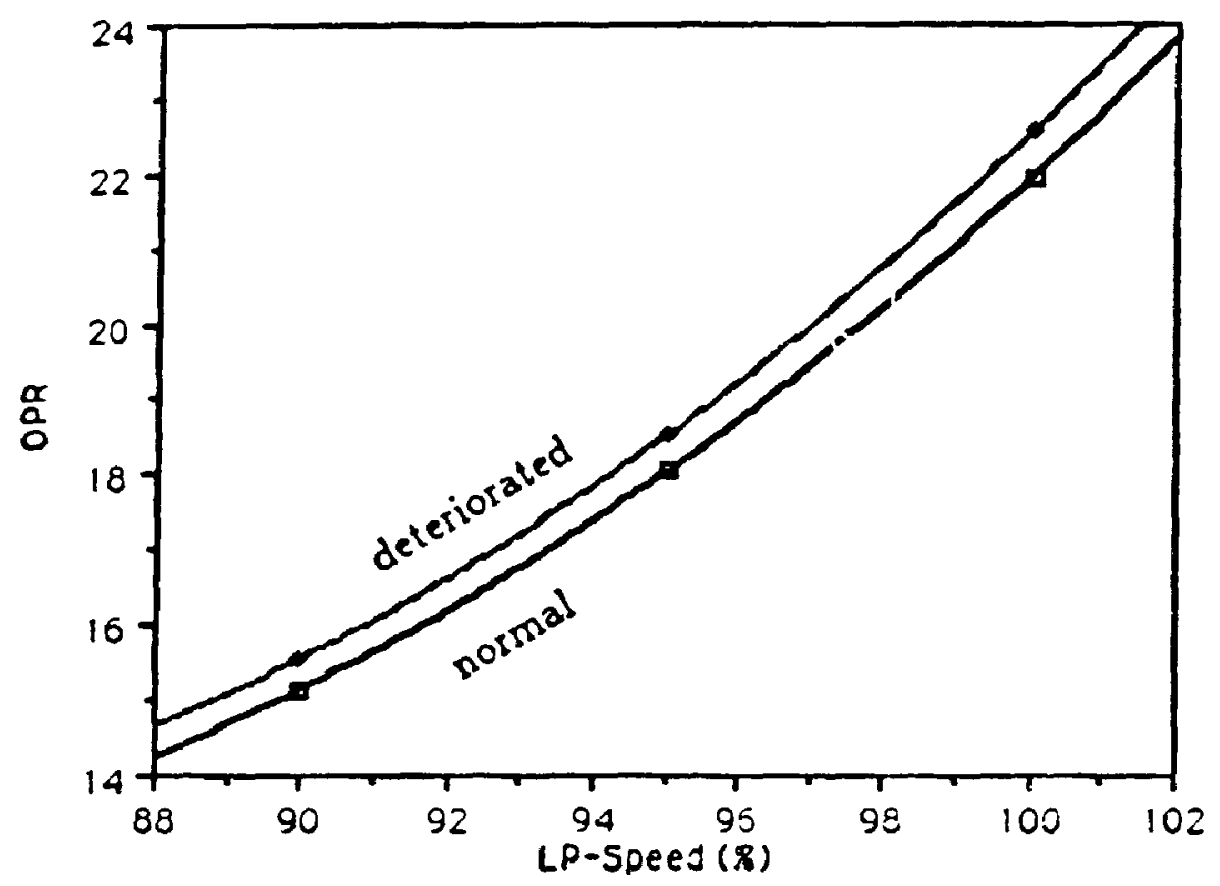

Deterioration 1: TIT VS LP-Speed

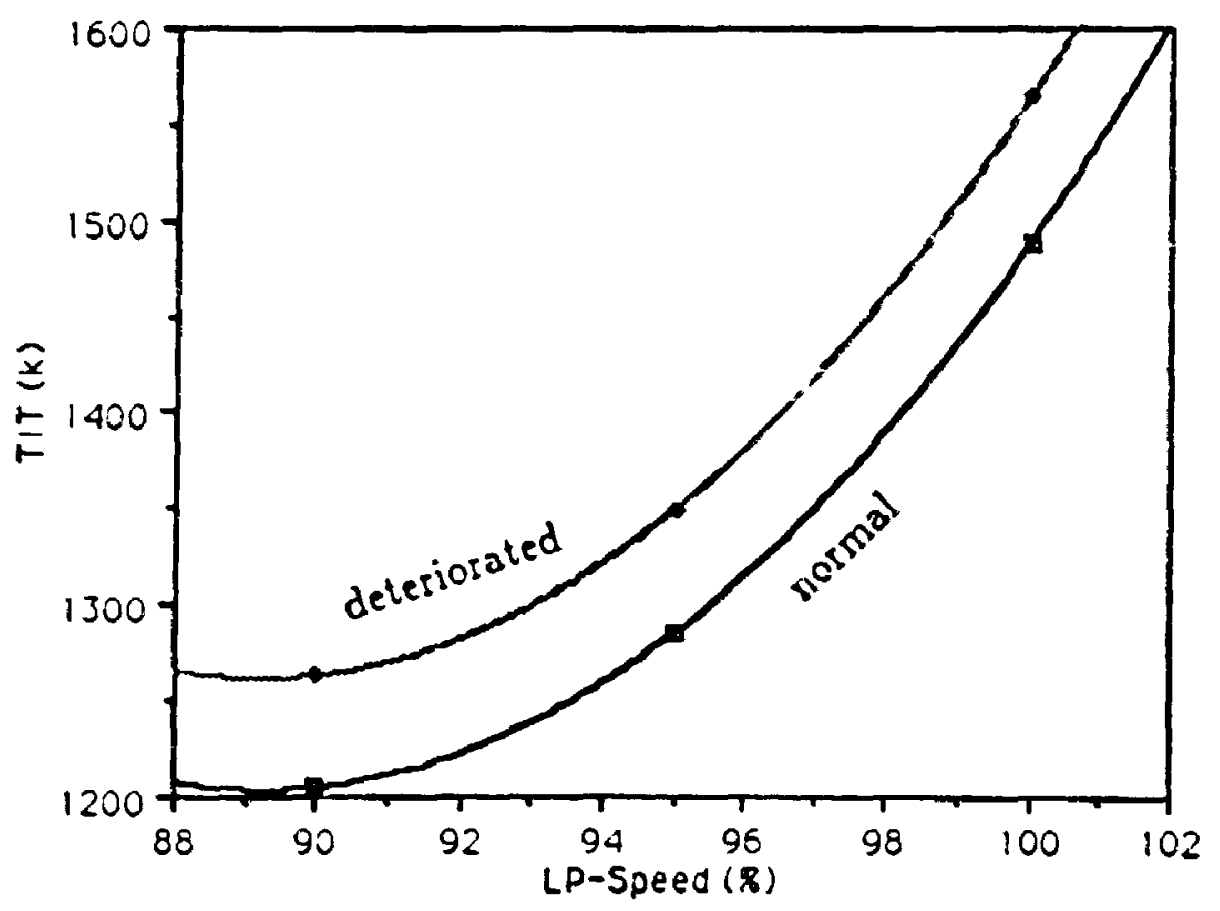

Fig 38--1: LPC Mechanical Damage 
Deterioration 1: Fuel Flow vs LP-Speed

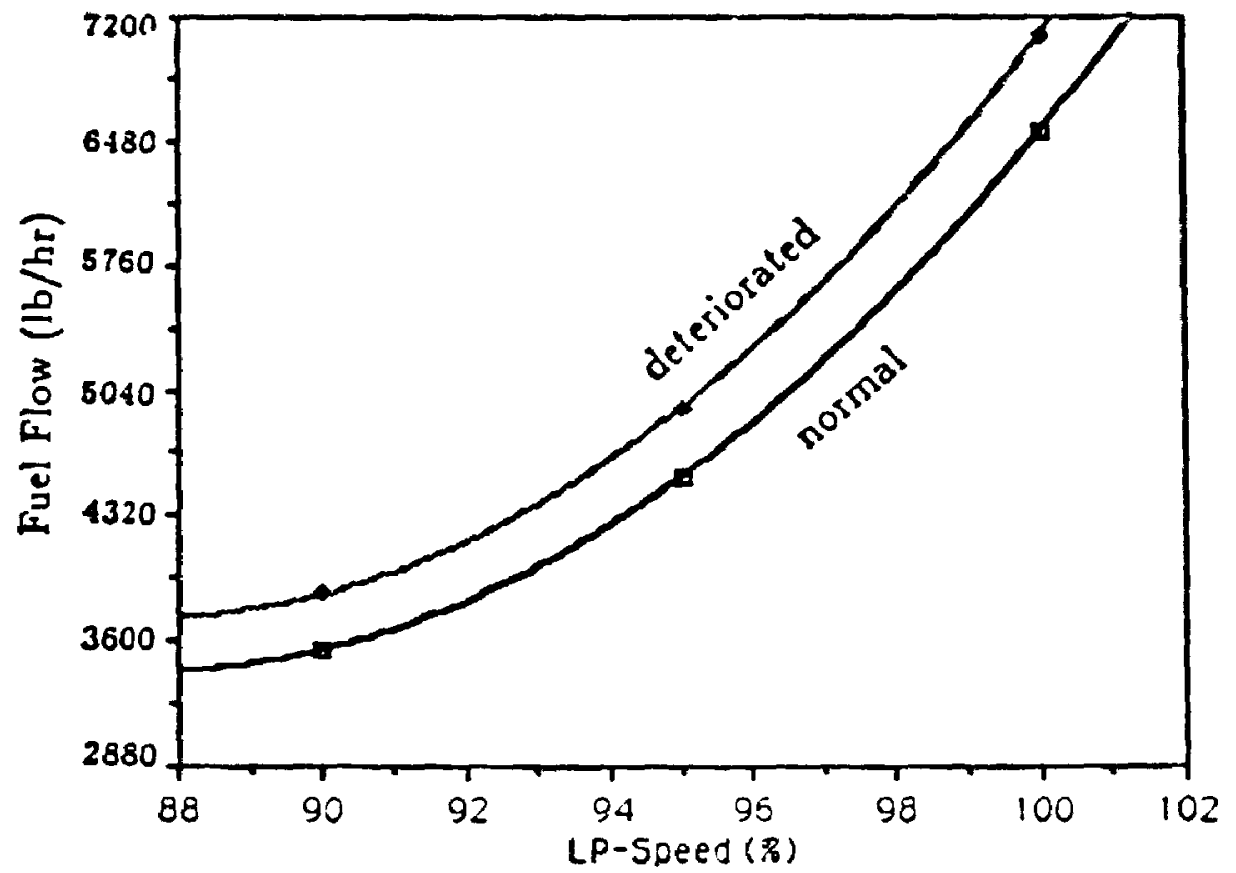

Deterioration 1: Wout VS LP-Speed

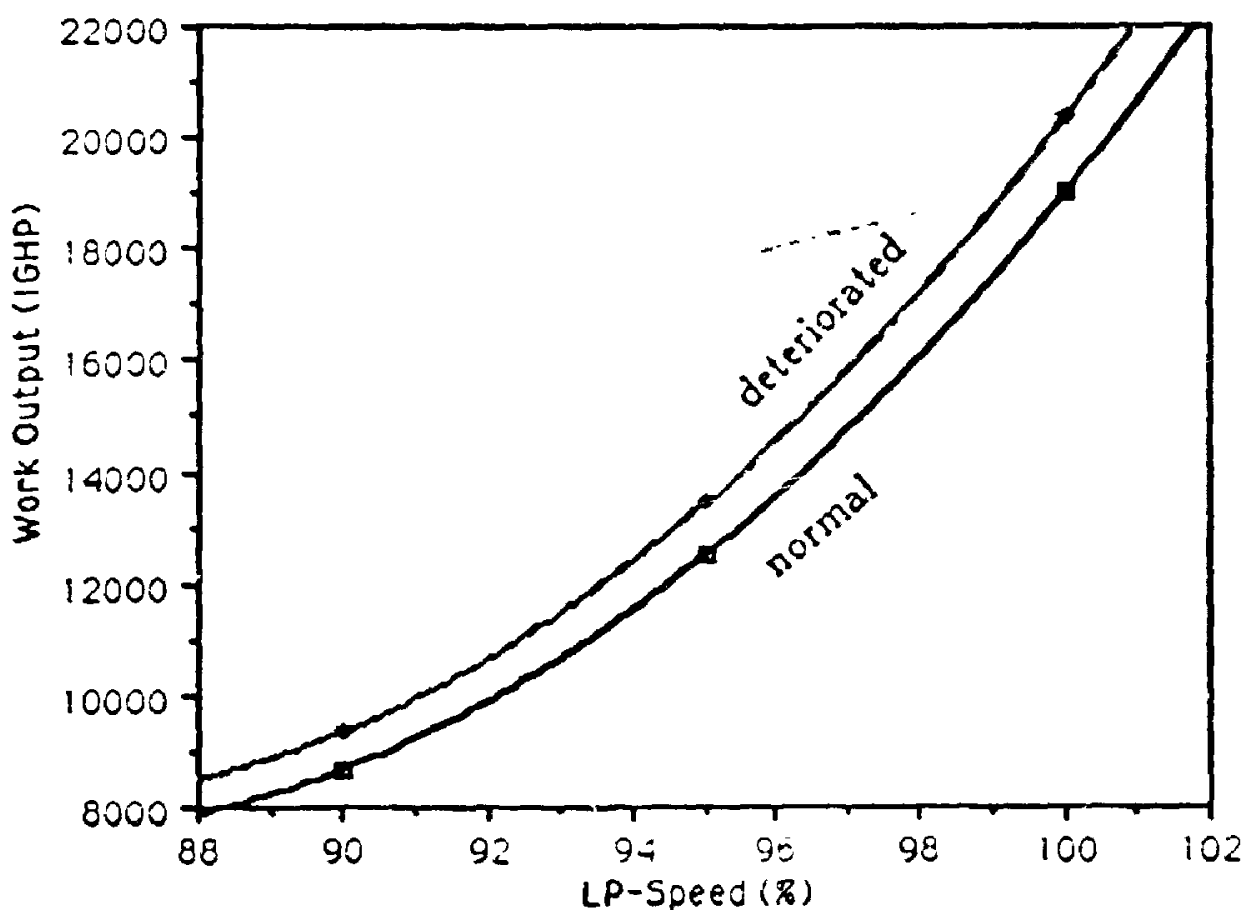

Fig 38--2 LPC Mechanical Damage 
Deterioration 2: OPR VS LP-Speed

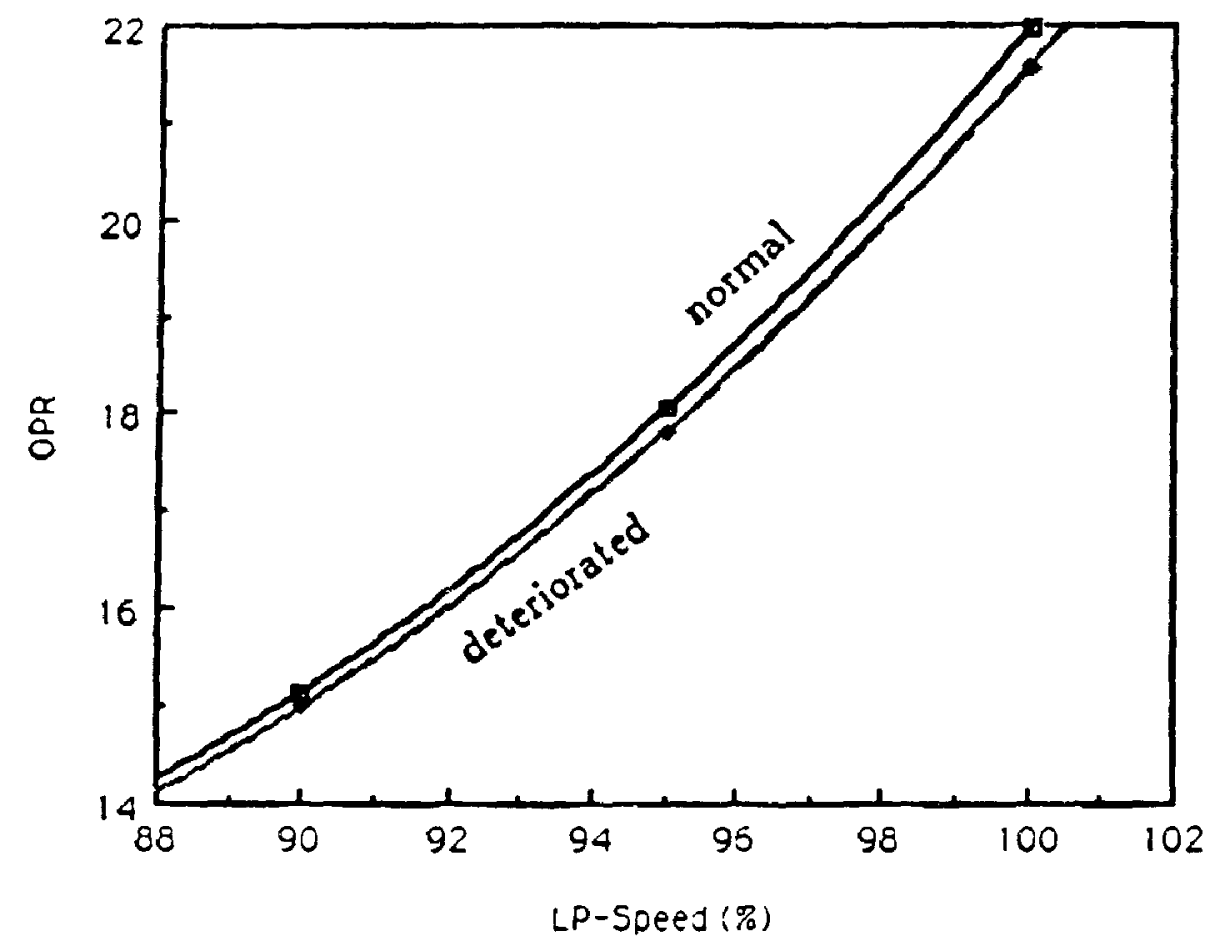

Deterioration 2: TIT VS LP-Speed

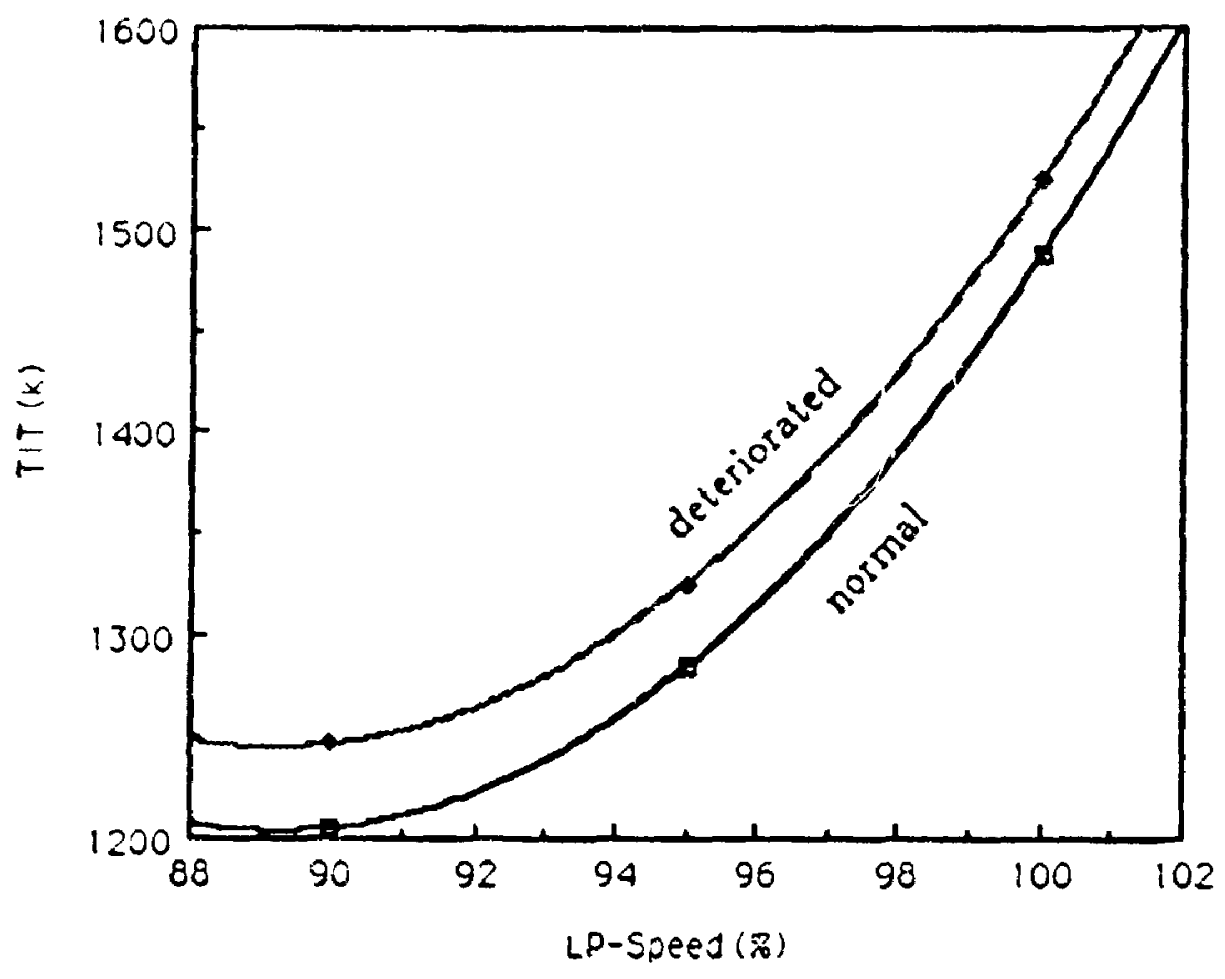

Fig 39--1: Compressor Excessive Leakage 
Deterioration 2: Fuel Flow vs LP-Speed

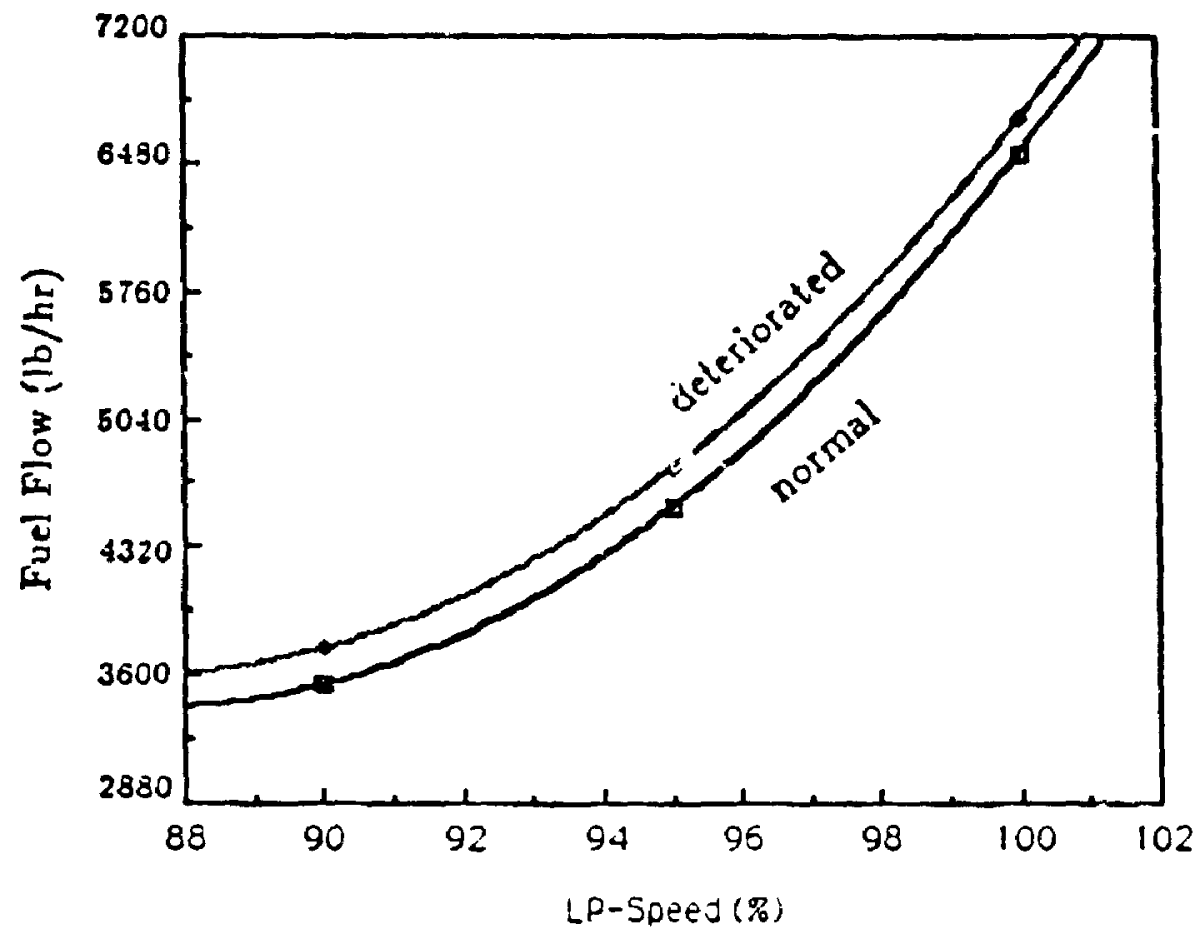

Deterioration 2: Wout vS LP-Speed

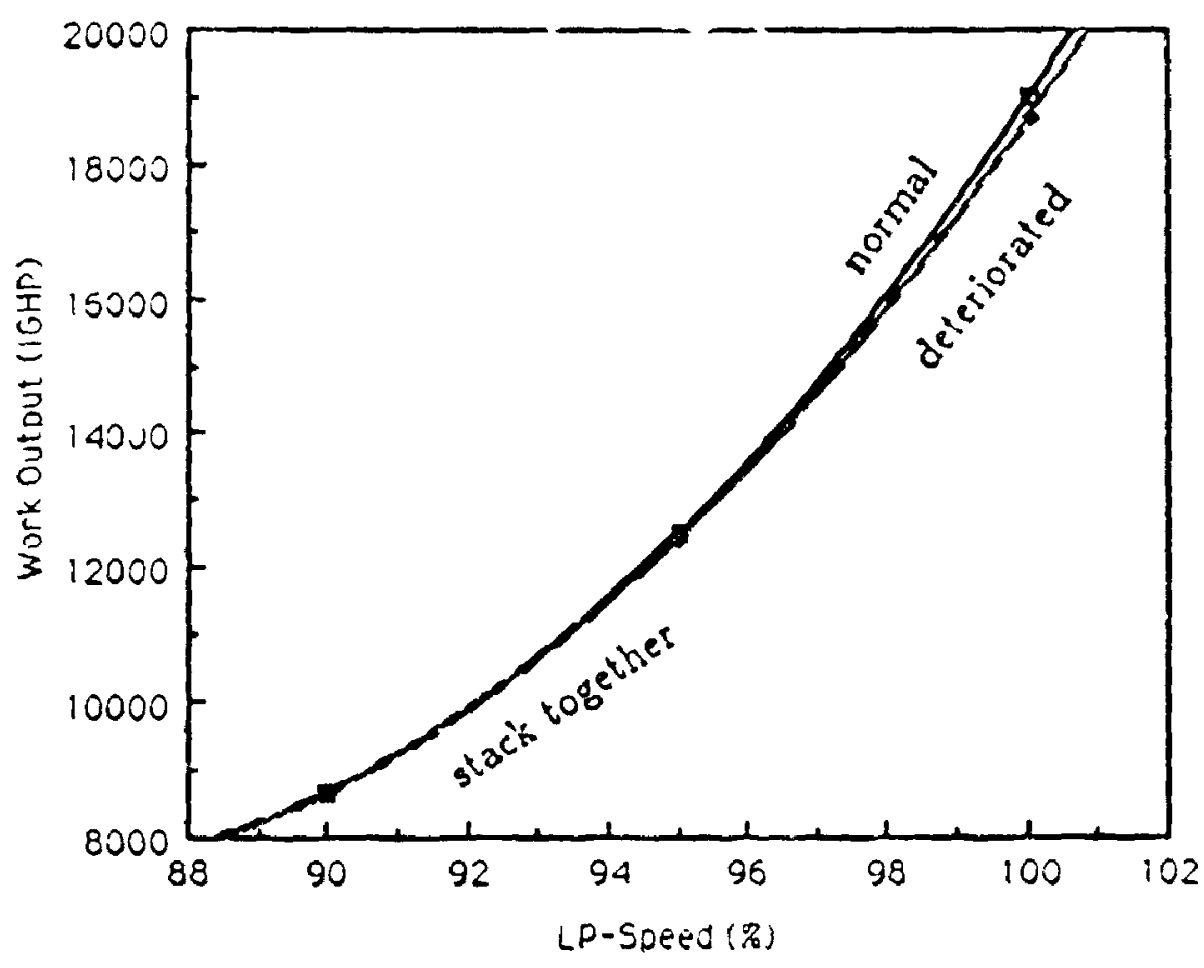

Fig 39--2. Compressor Excessive Leakage 
Deterioration 3: OPR VS LP-Speed

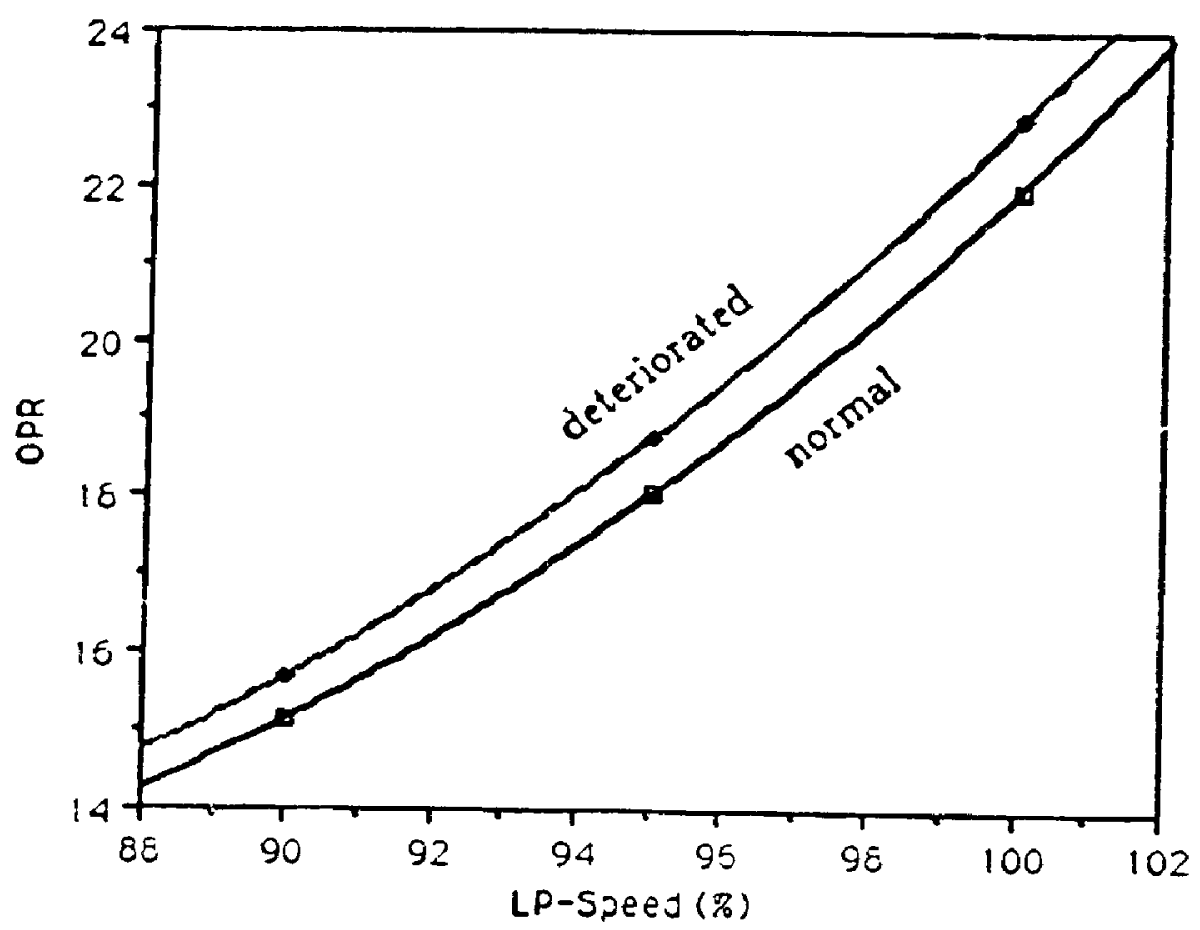

Deterioration 3: TIT VS LP-Speed

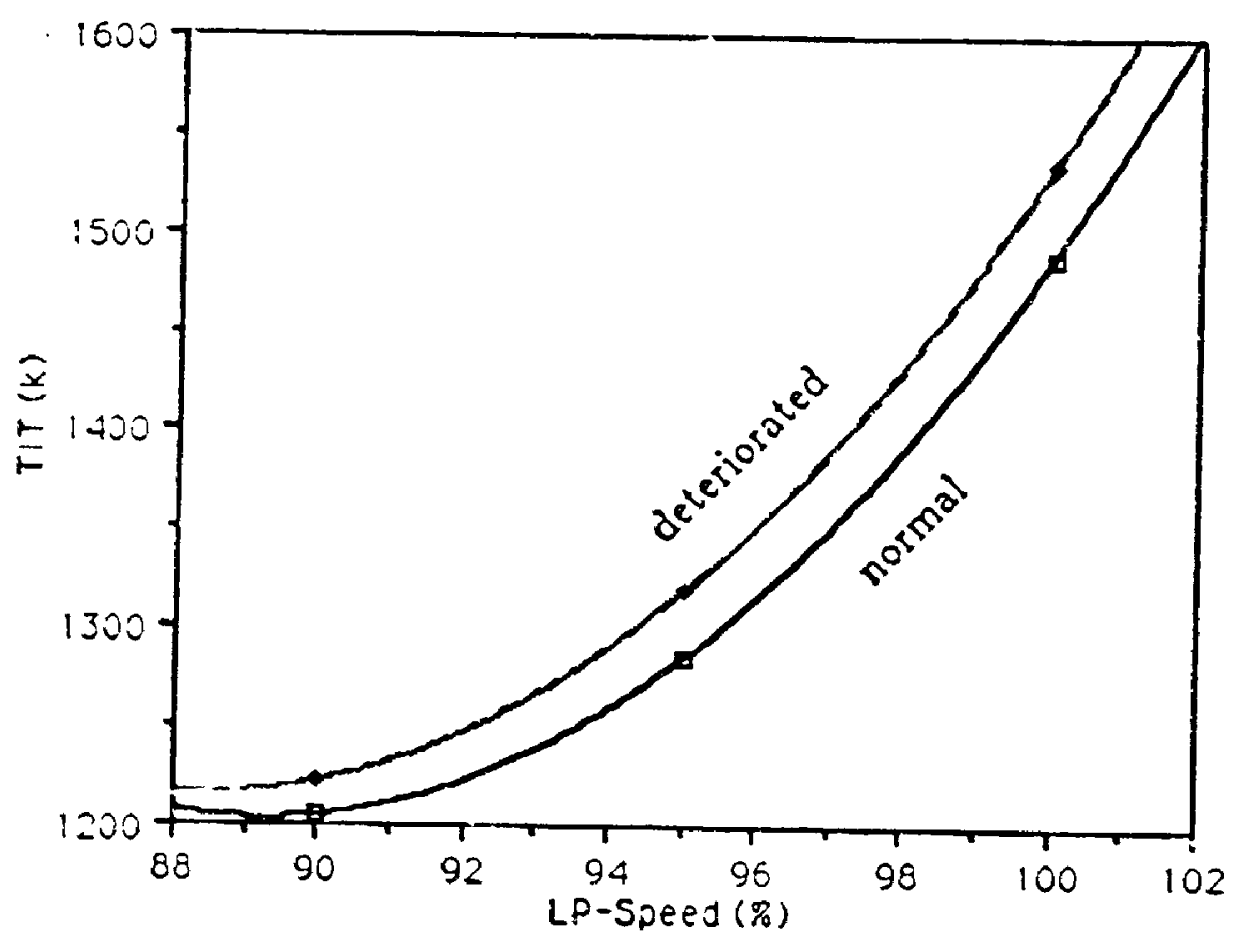

Fig 40--1: HPT Mechanical Damage 
Deterioration 3: Fuel Flow vS LP-Speed

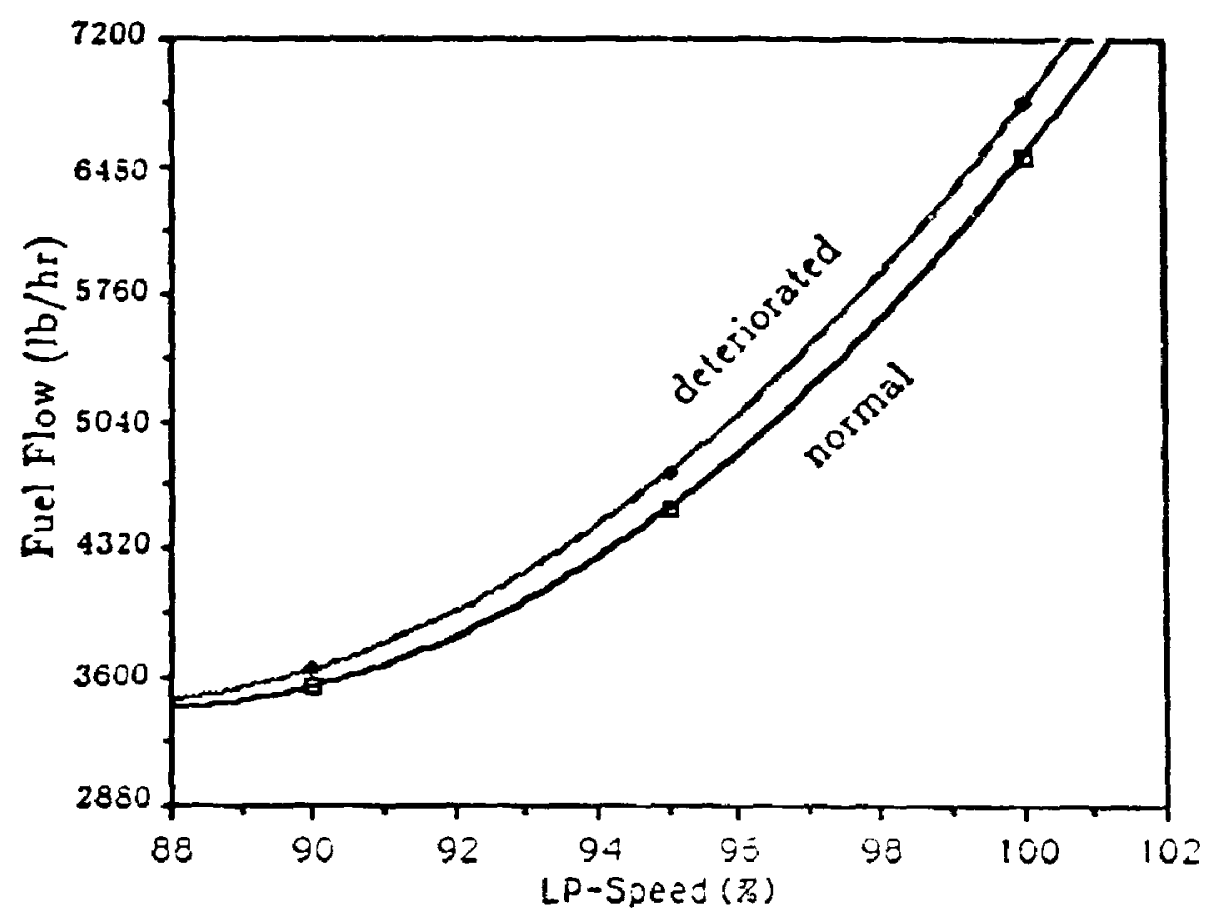

Deterioration 3: Wout vS LP-Speed

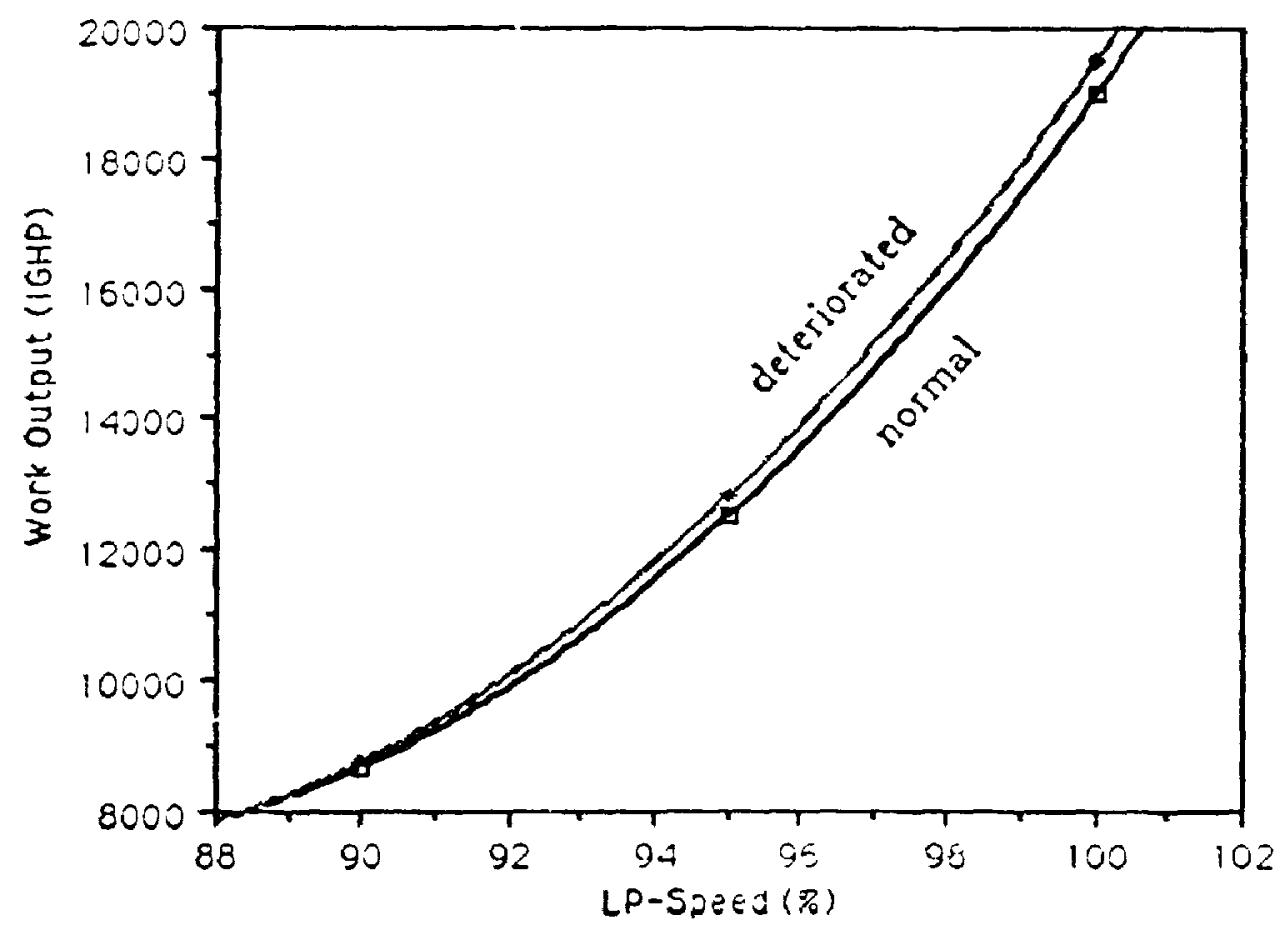

Fig 40--2 HPT Mecnanical Damage 
Deterioration 4: OPR VS LP-Speed

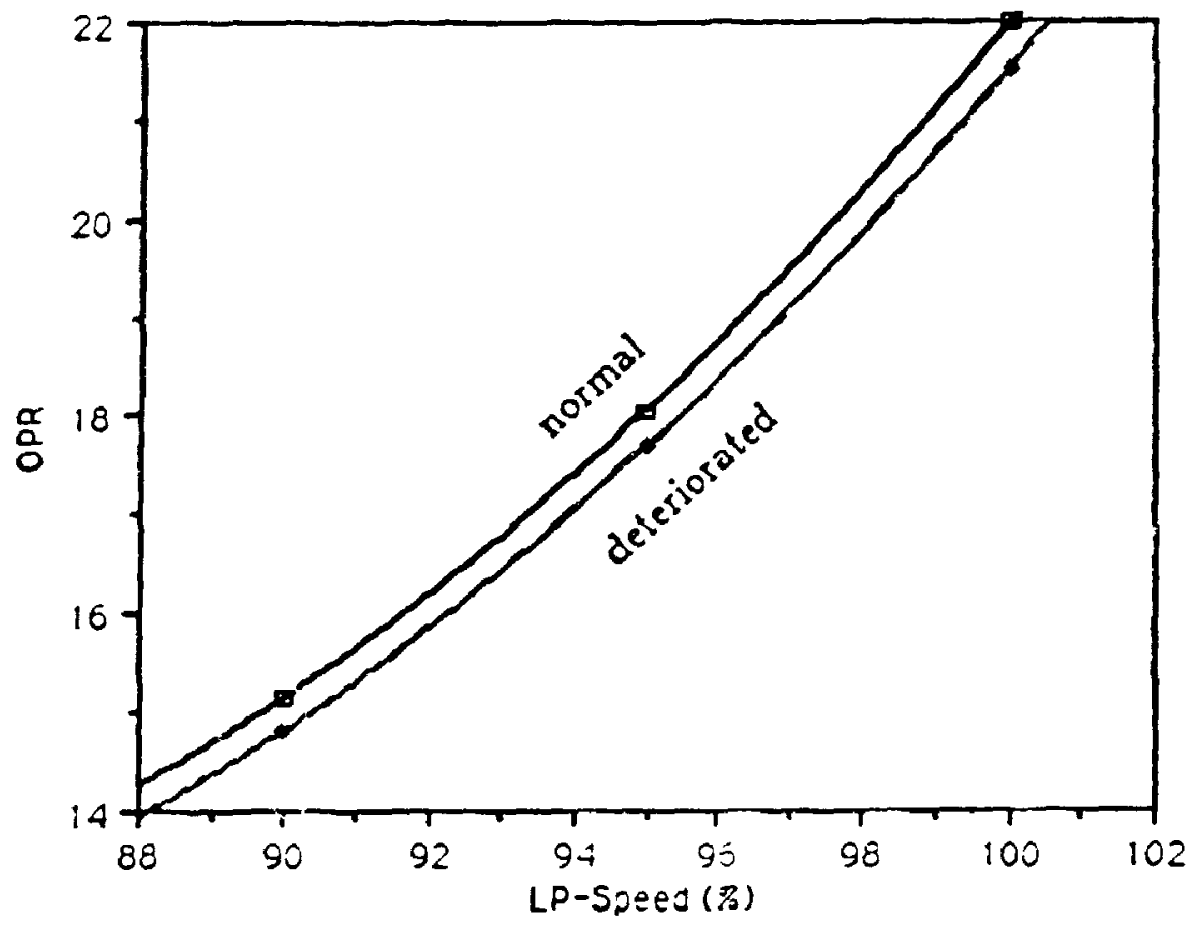

Deterioration 4: TIT VS LP-Speed

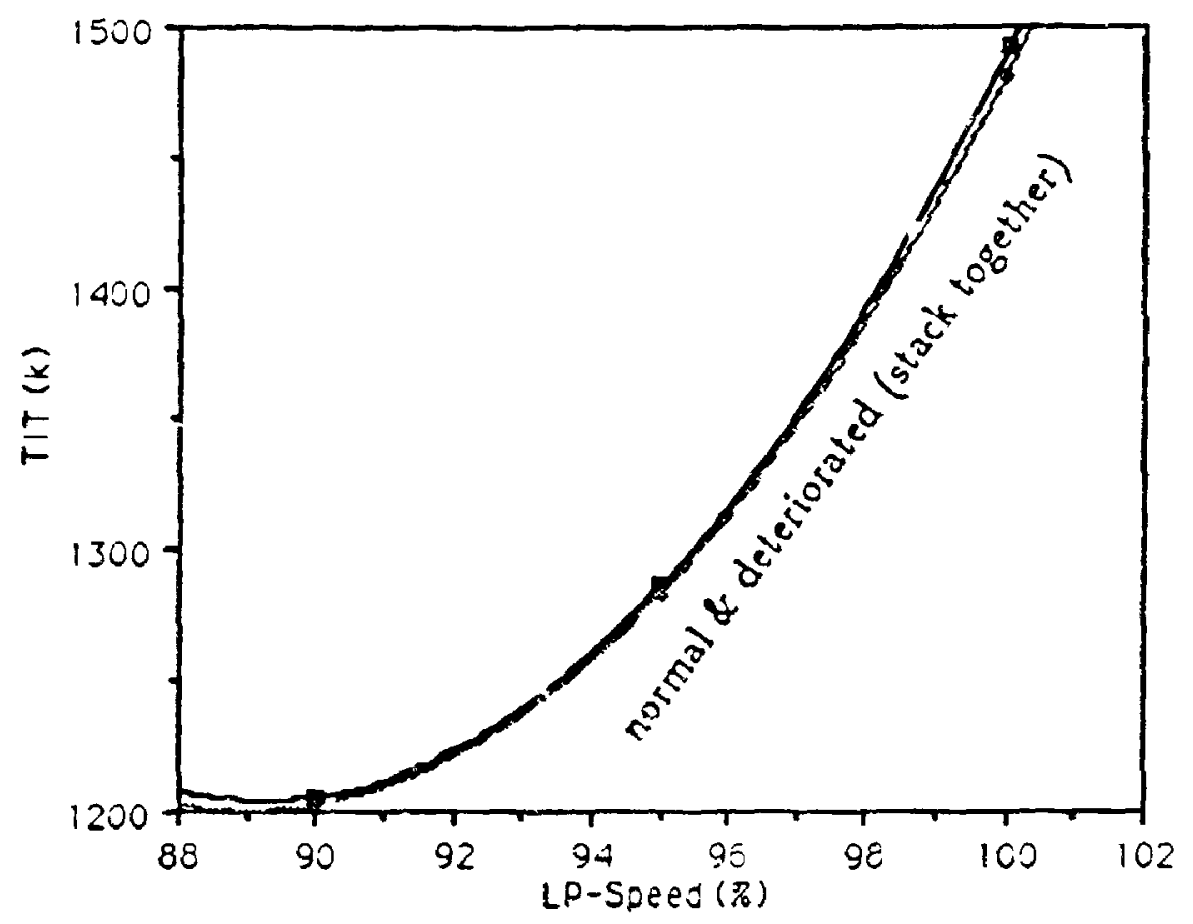

Fig. 41--1. HFT ERosion 
Deterioration 4: Fuel Flow vs LP-Speed

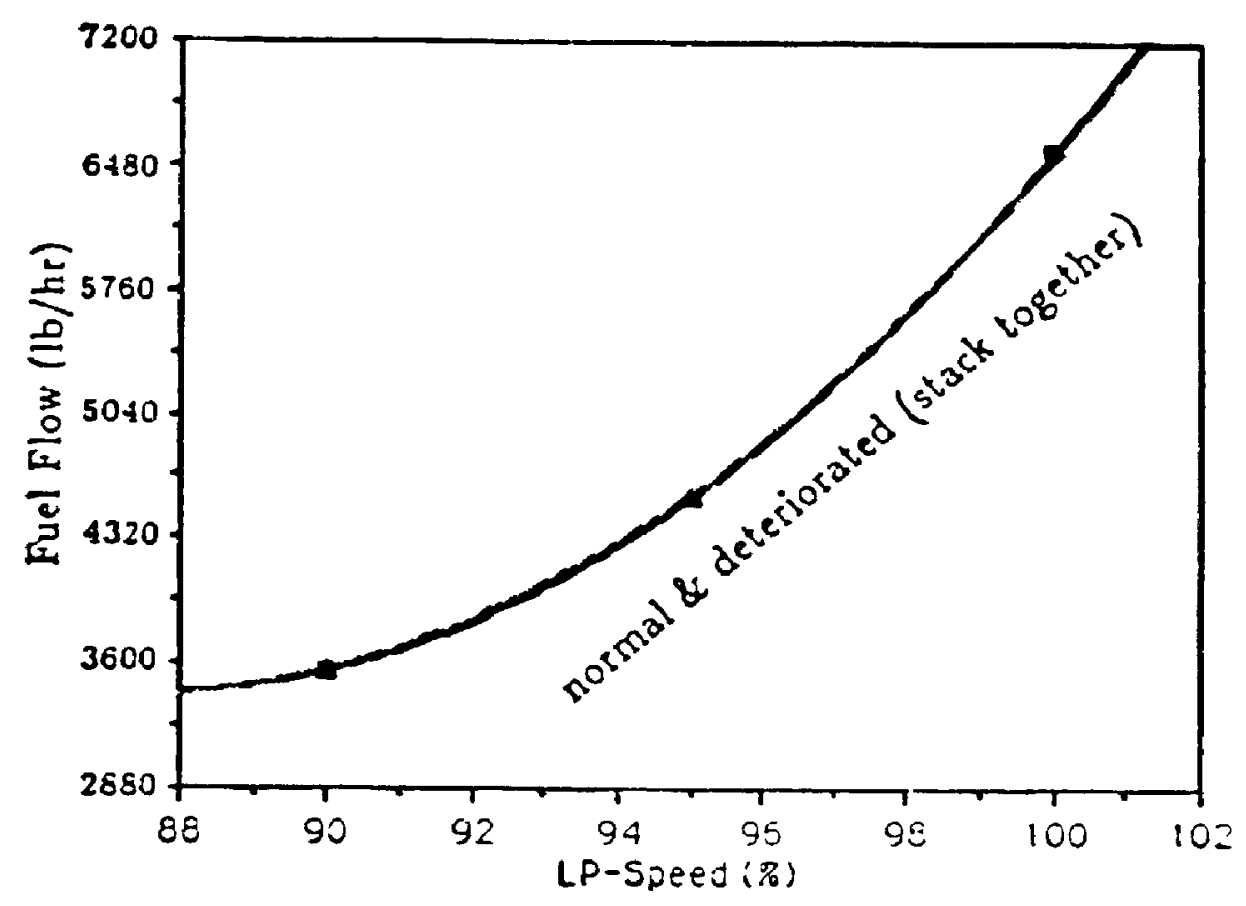

Deterioration 4. Wout vS LP-Speed

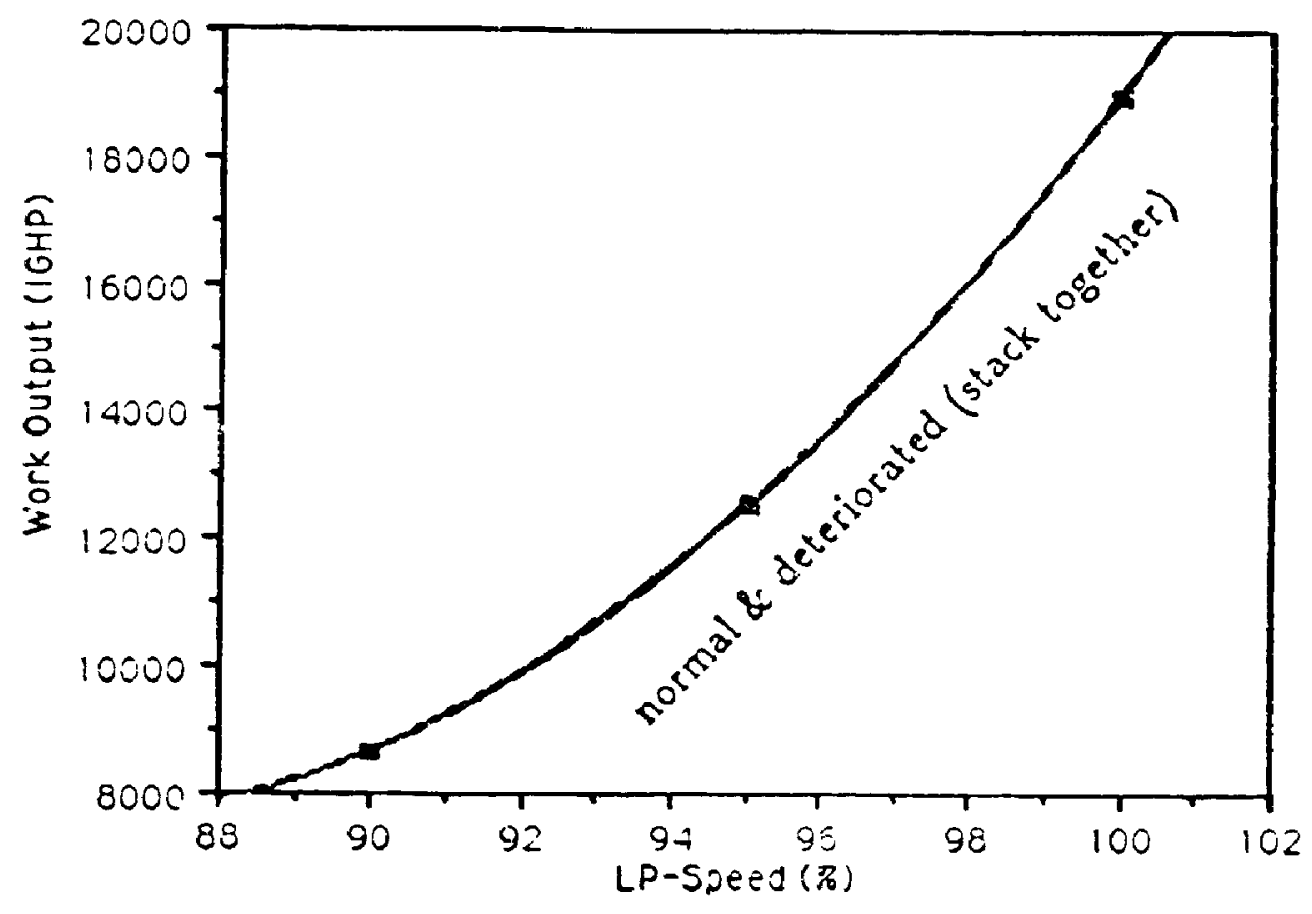

Fig. $4 !--2$. HPT Erosion 
Deterioration 5: OPR VS LP-Speed

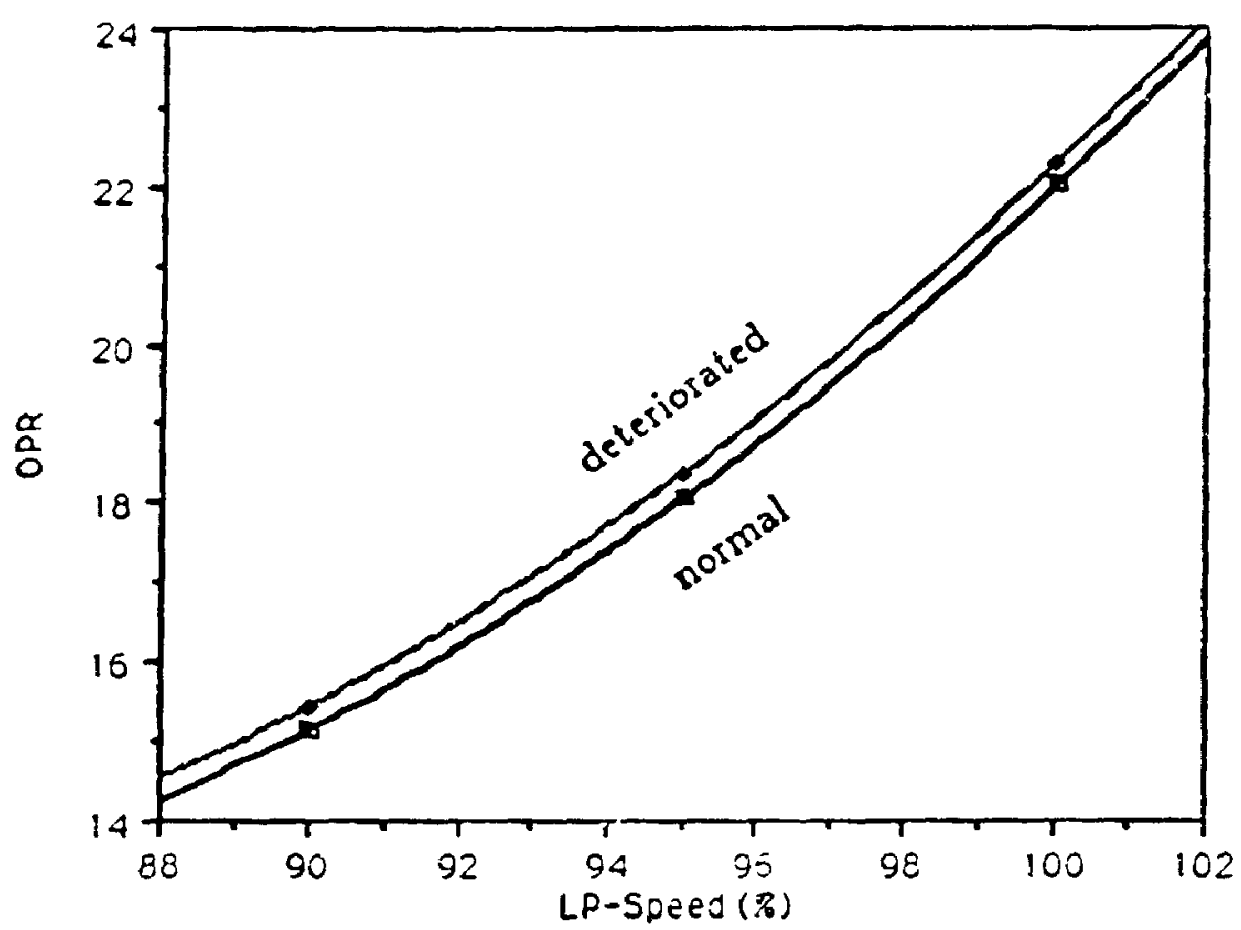

Deterioration 5: TIT VS LP-Speed

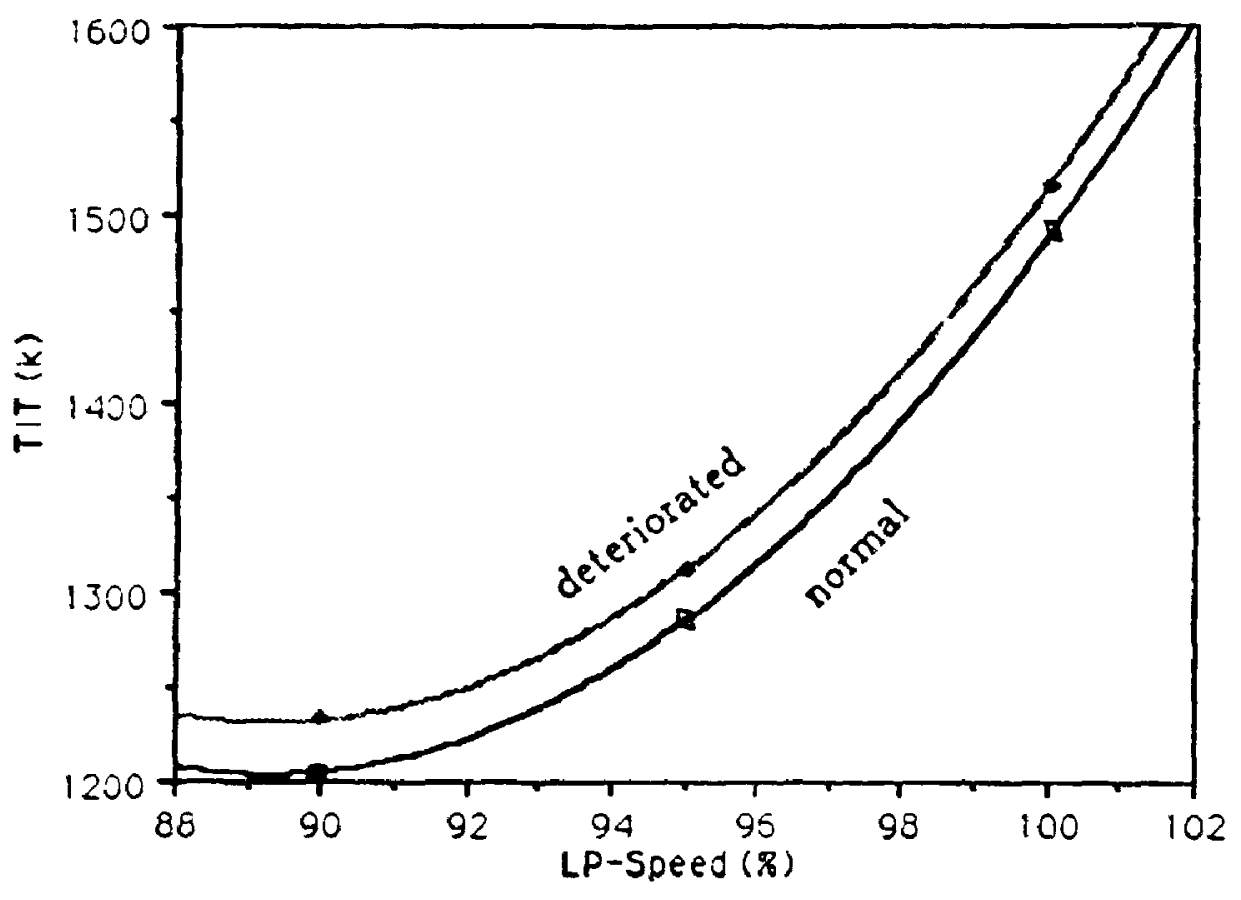

Fig 42--1. LPT Erosion 
Deterioration 5: Fuel Flow vs LP-Speed

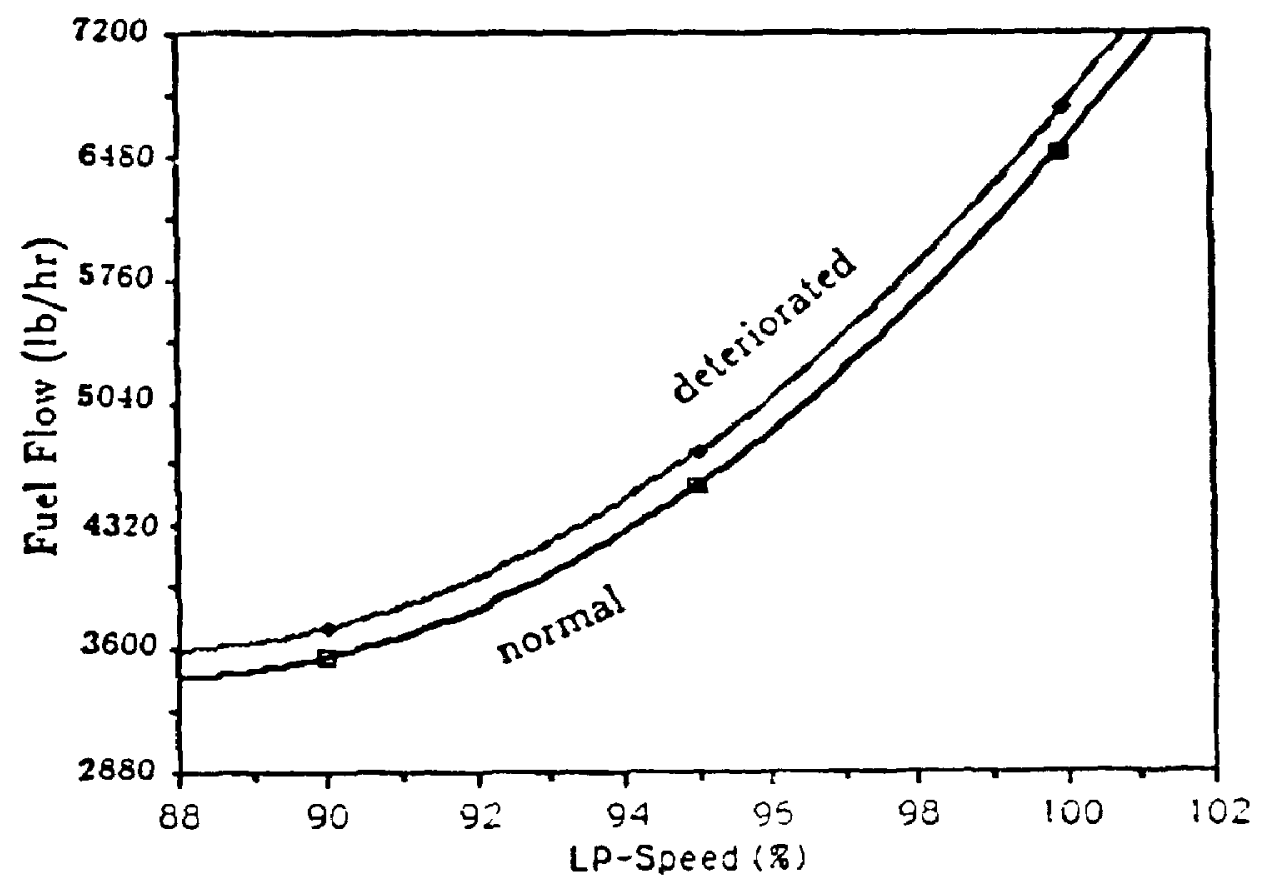

Deterioration 5. Wout vs LP-Speed

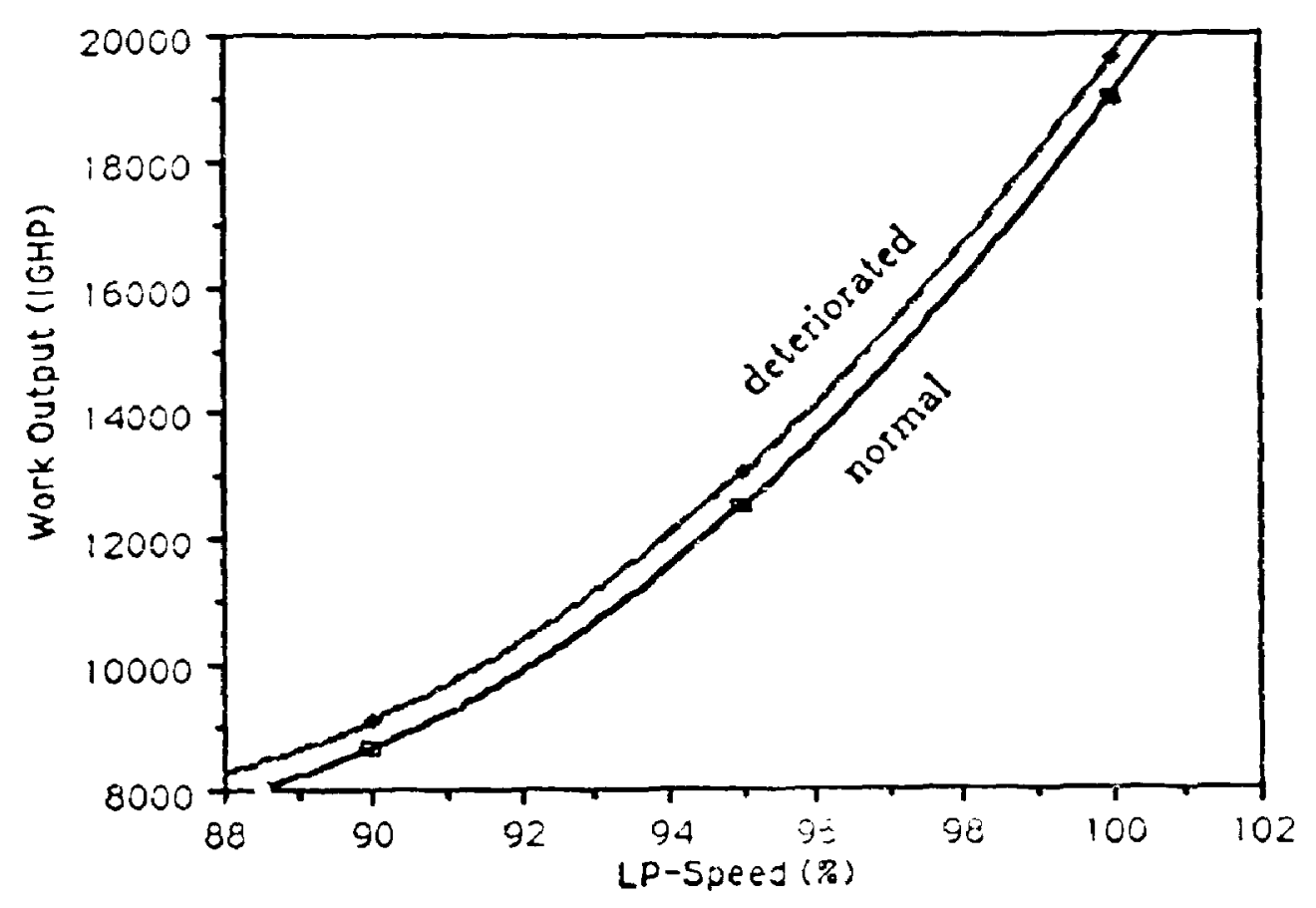

FIg 42--2 LFTEROSion 


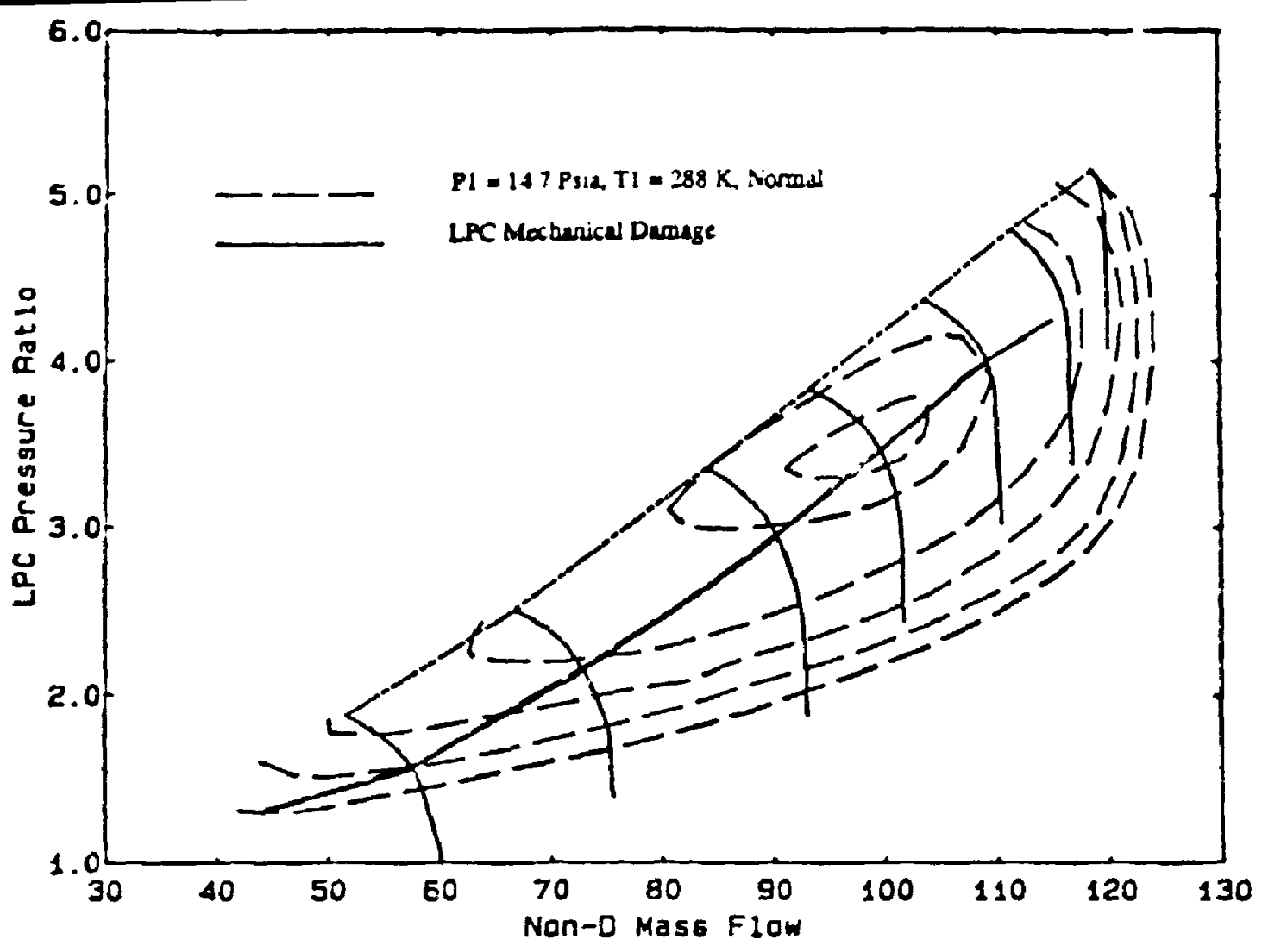

Fig. 43 (1) Effect of LPC Mechanical Damage on LPC Running Line

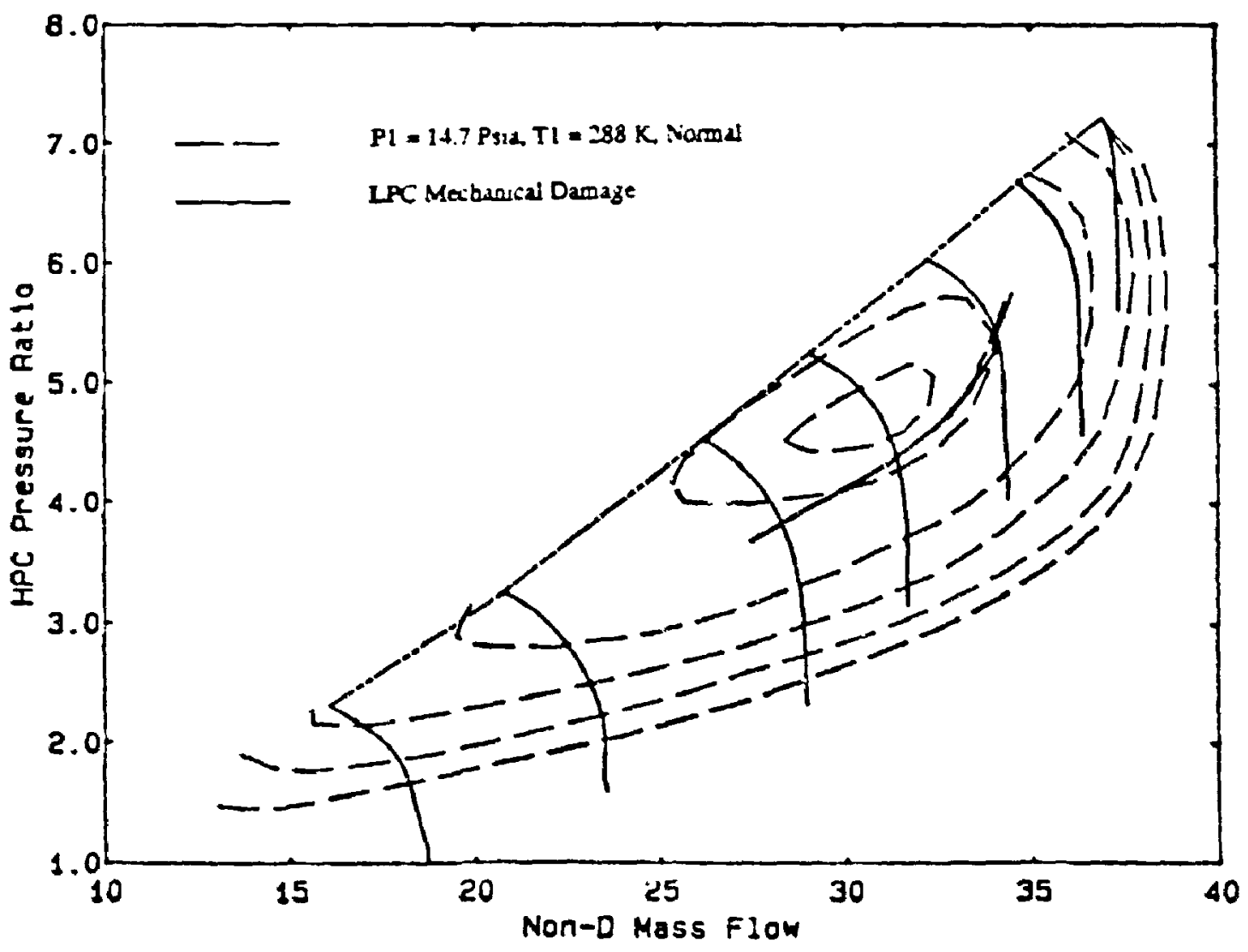

Fig. 4 (1) Effect of LPC Mechanical Damage on HPC Running Line 


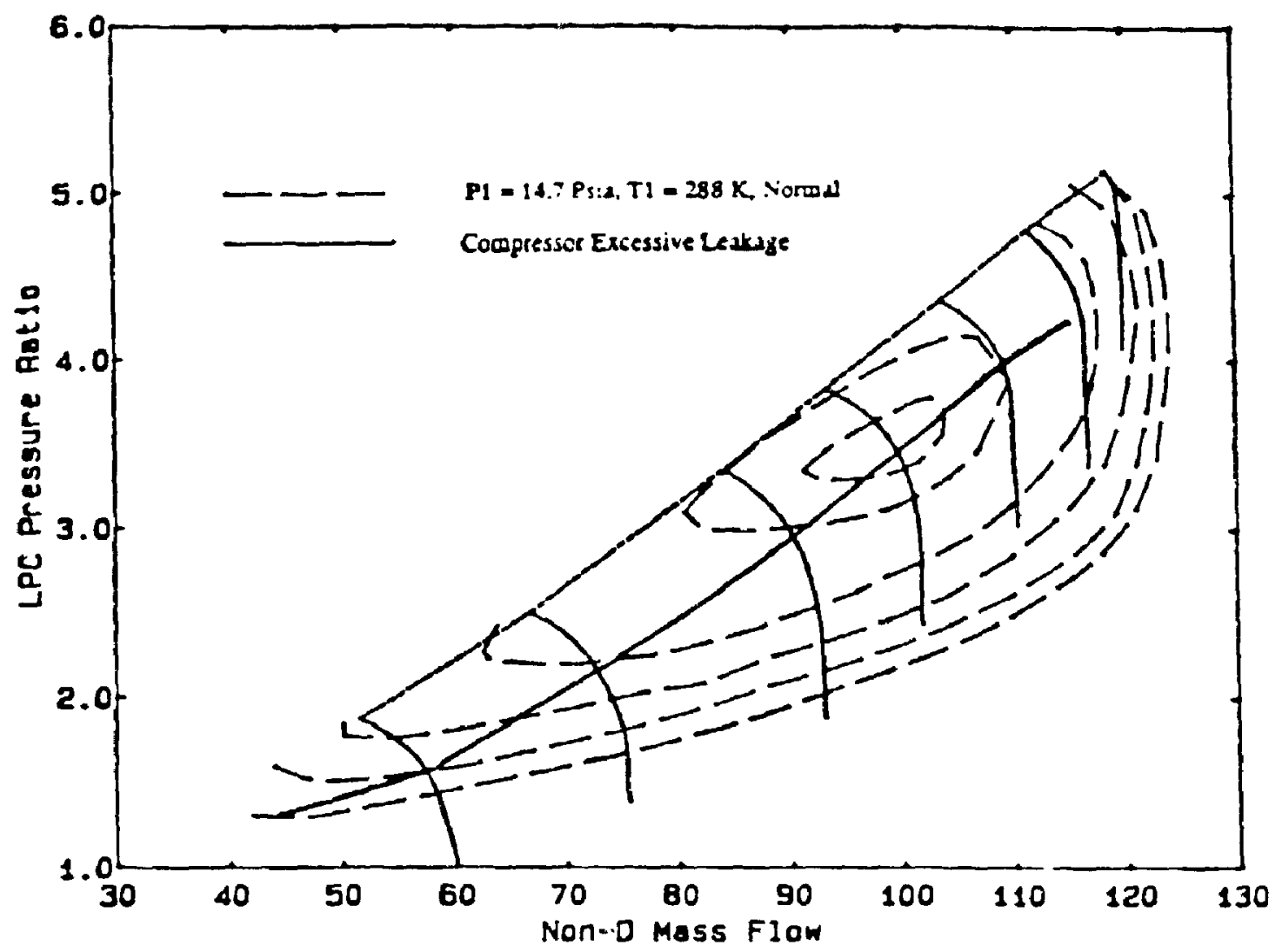

Fig. 43 (2) Effect of Compressor Excessive Leakage on LPC Running Line

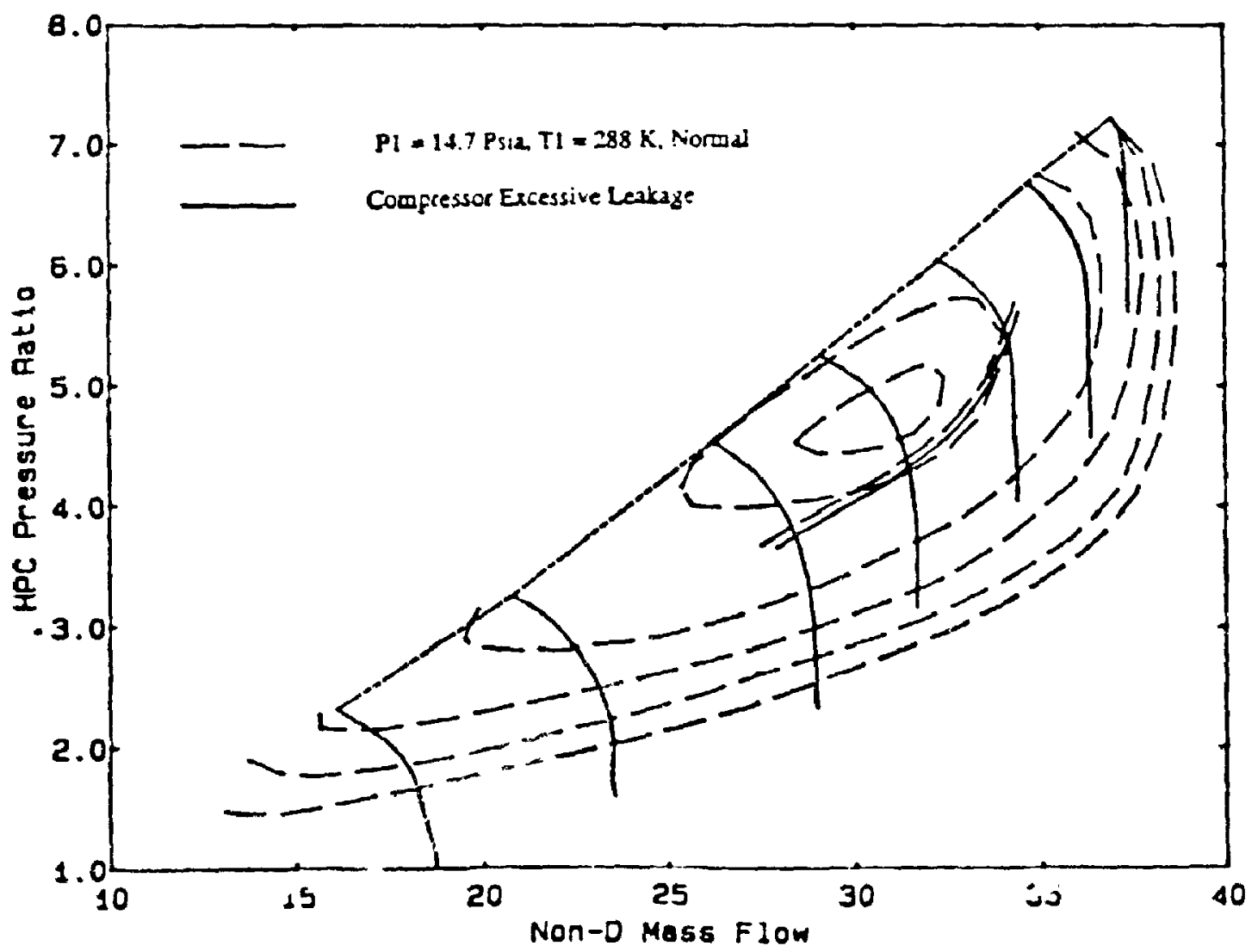

Fig. 44 (z) Effect of Compressor Excessive Leakage on HPC Running Line 


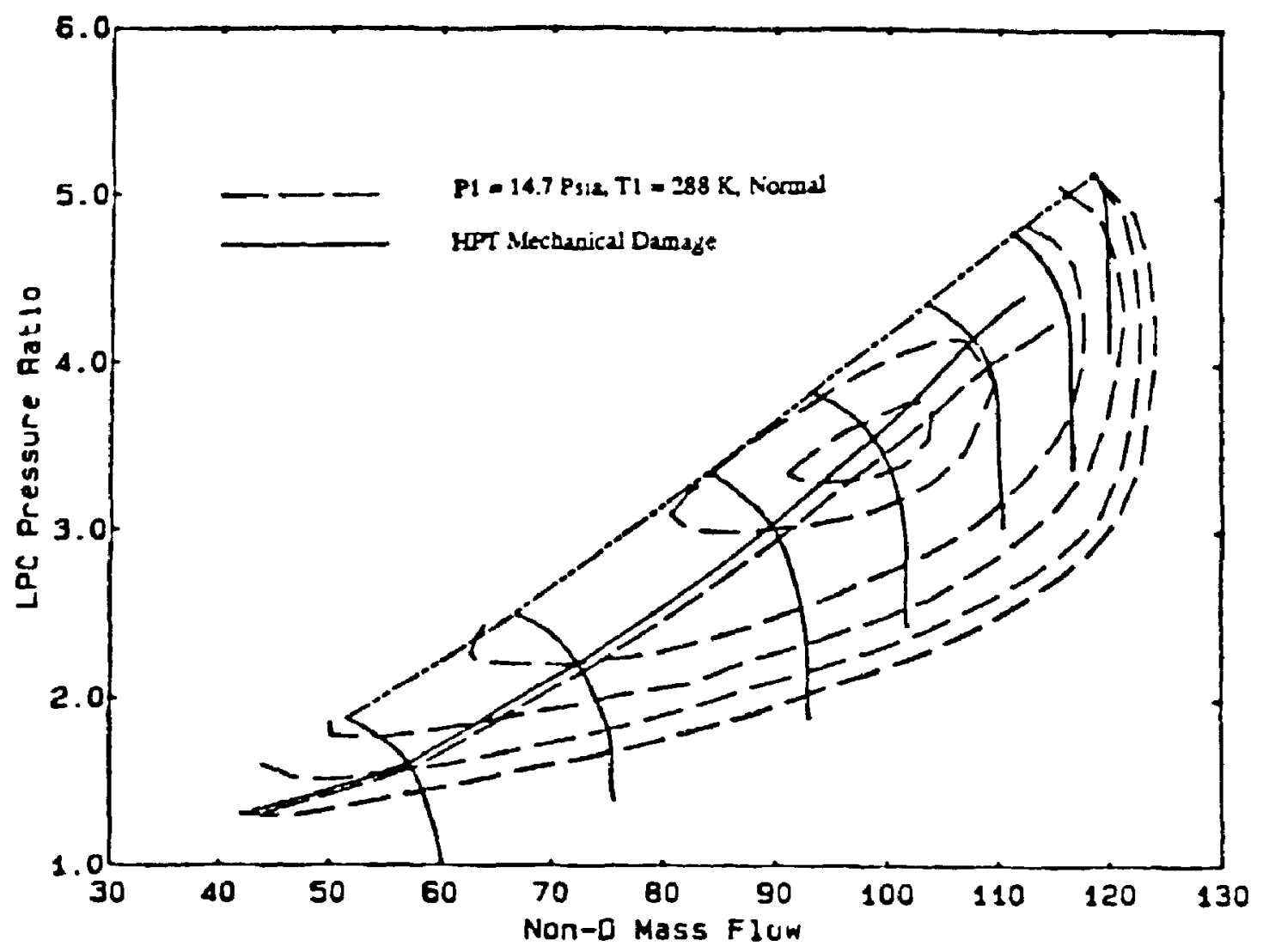

Fig. 43 (3) Effect of HPT Mechanical Damage on LPC Running Line

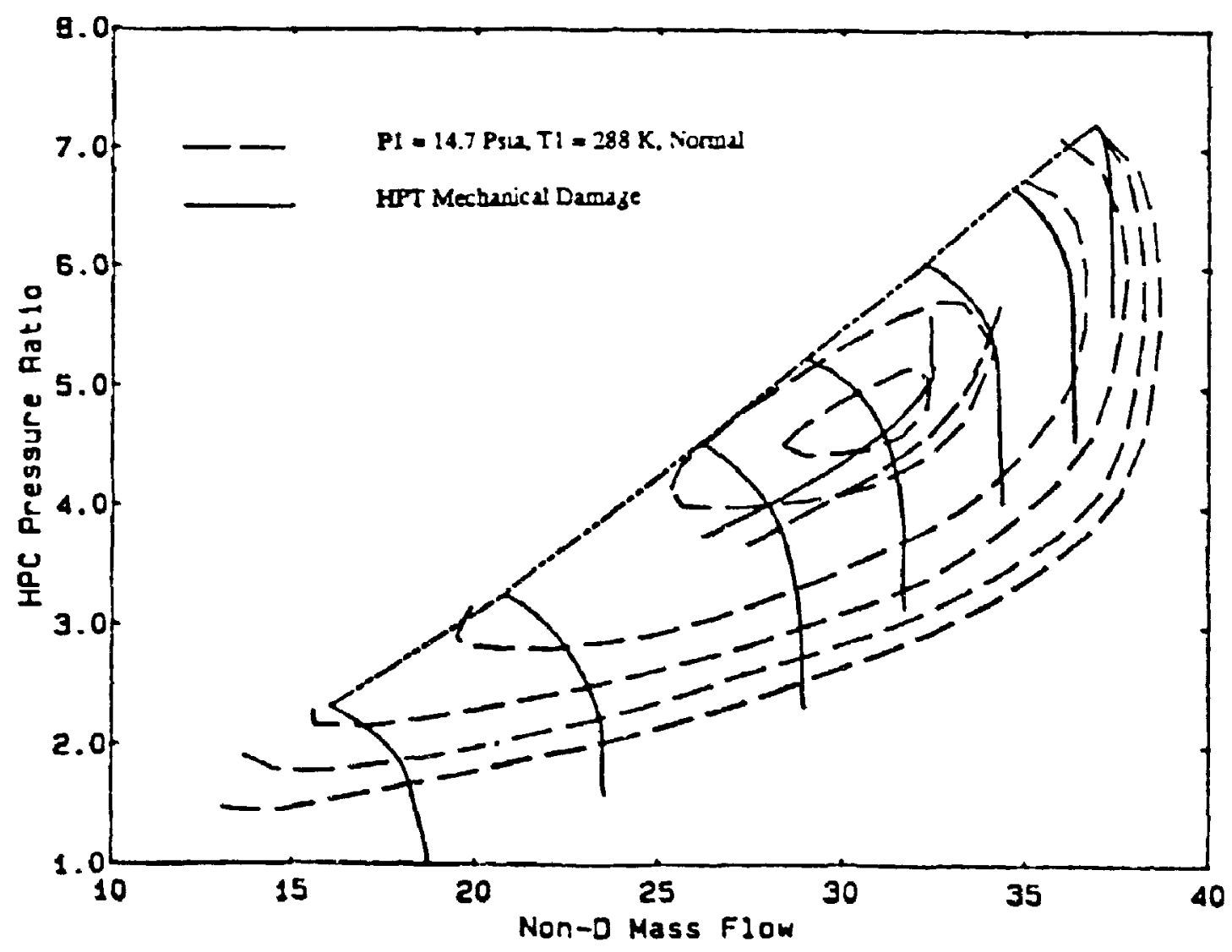

Fig. 4 (3) Effect of HPT Mechanical Damage on HPC Running Line 


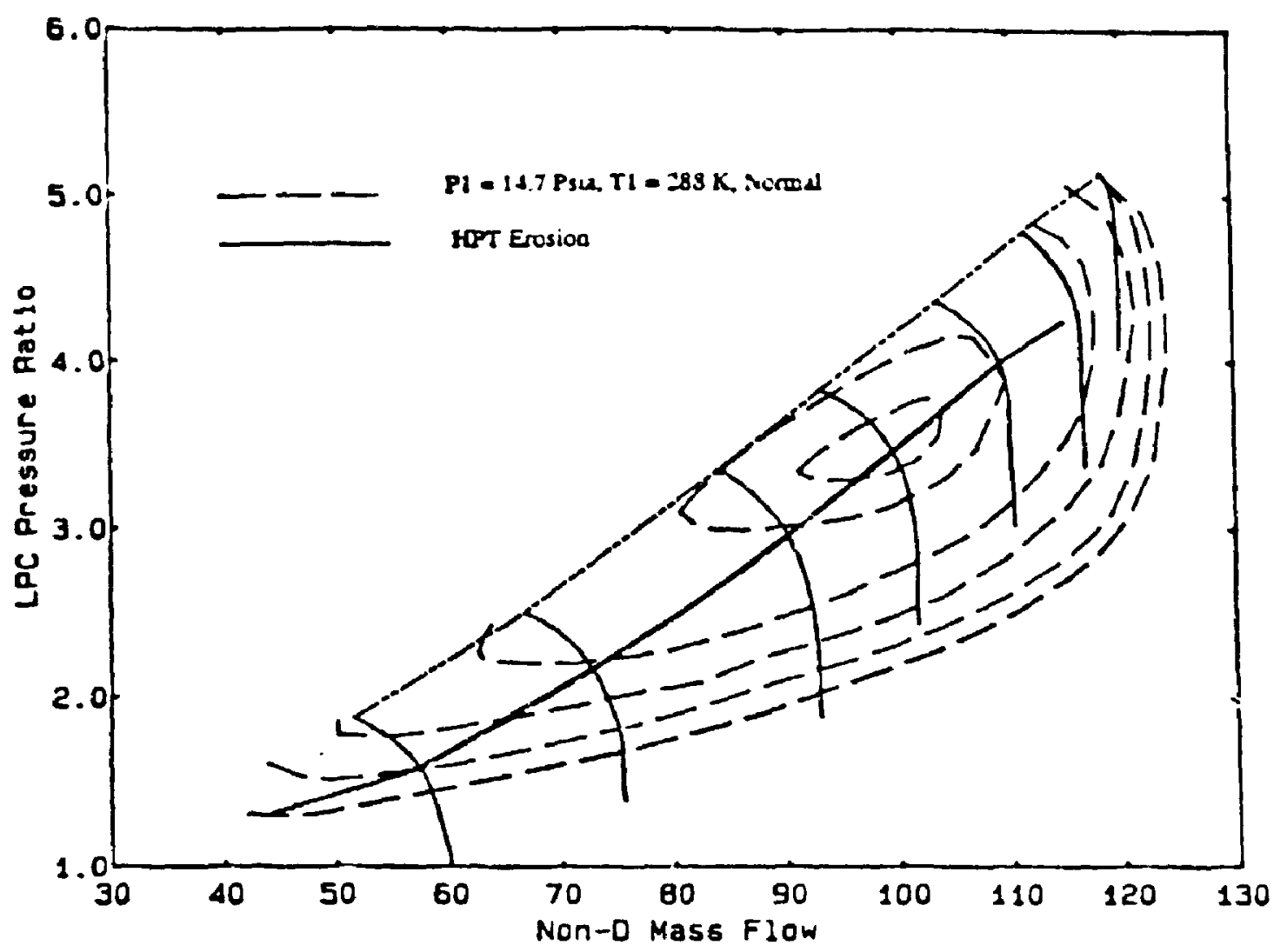

Fig. 43 (4) Effect of HPT Erosion on LPC Running Line

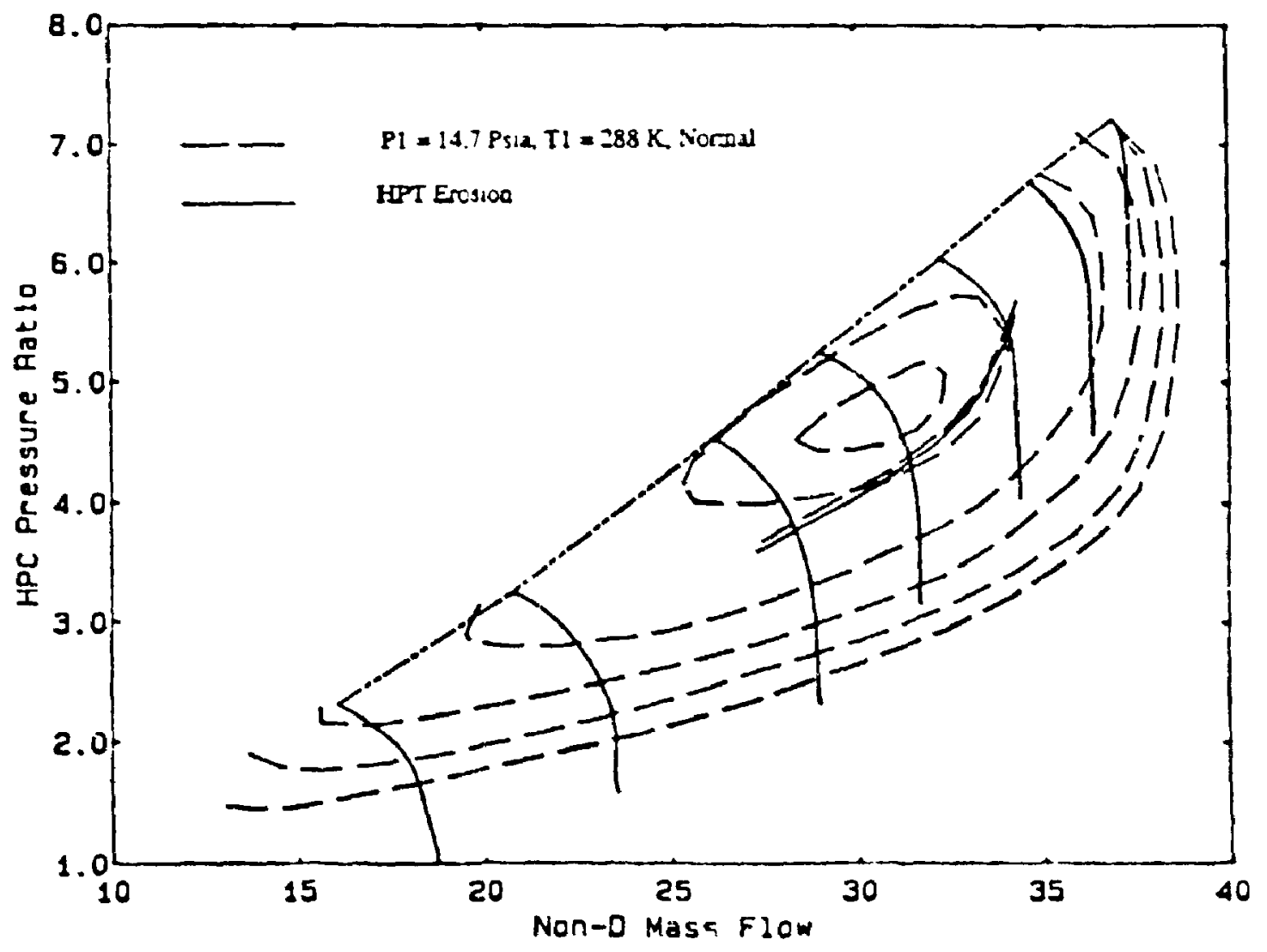

Fig. 44 (4) Effect of HPT Erosion on HPC Running Line 


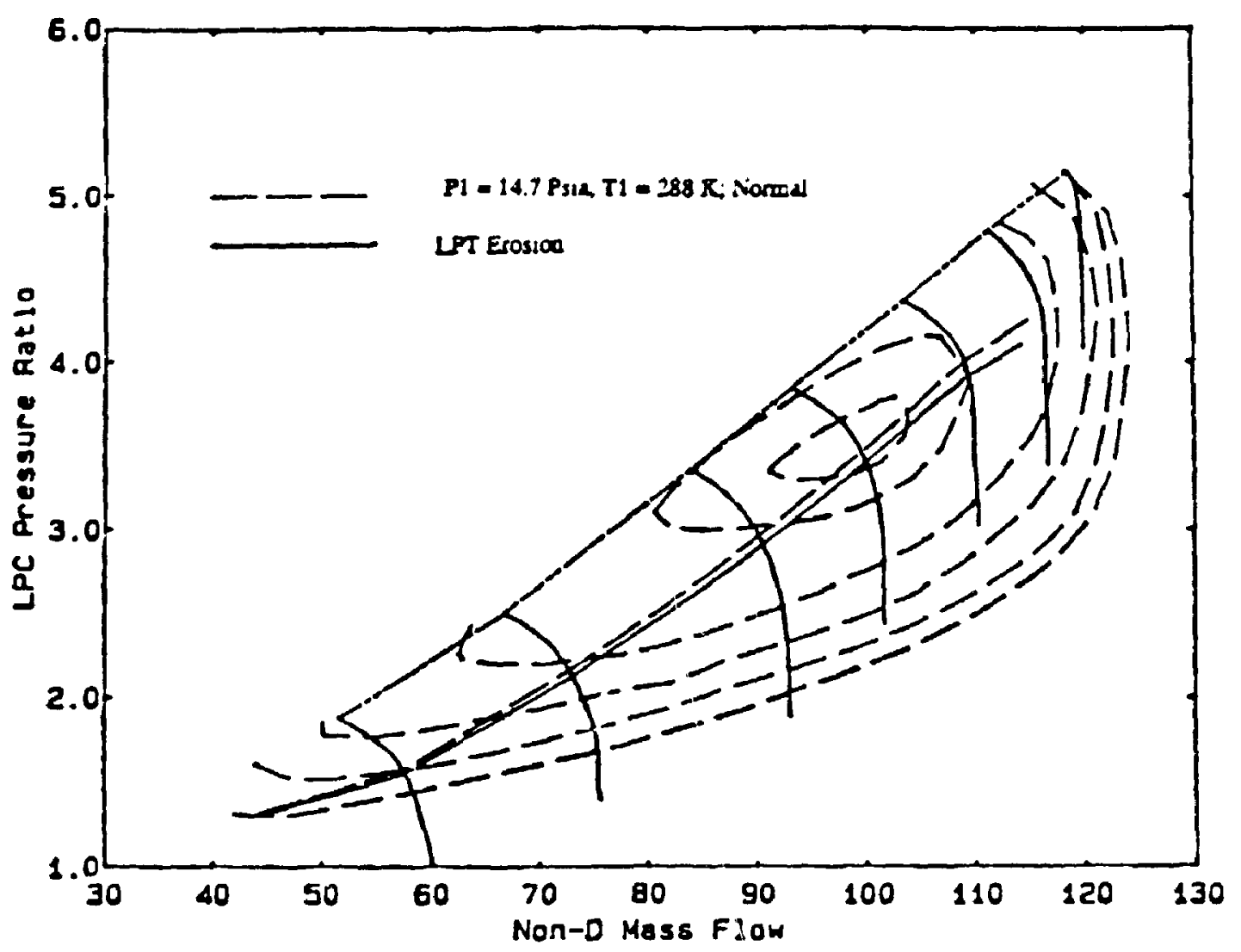

Fig. 43 (5) Effect of LPT Erosion wn LPC Running Line

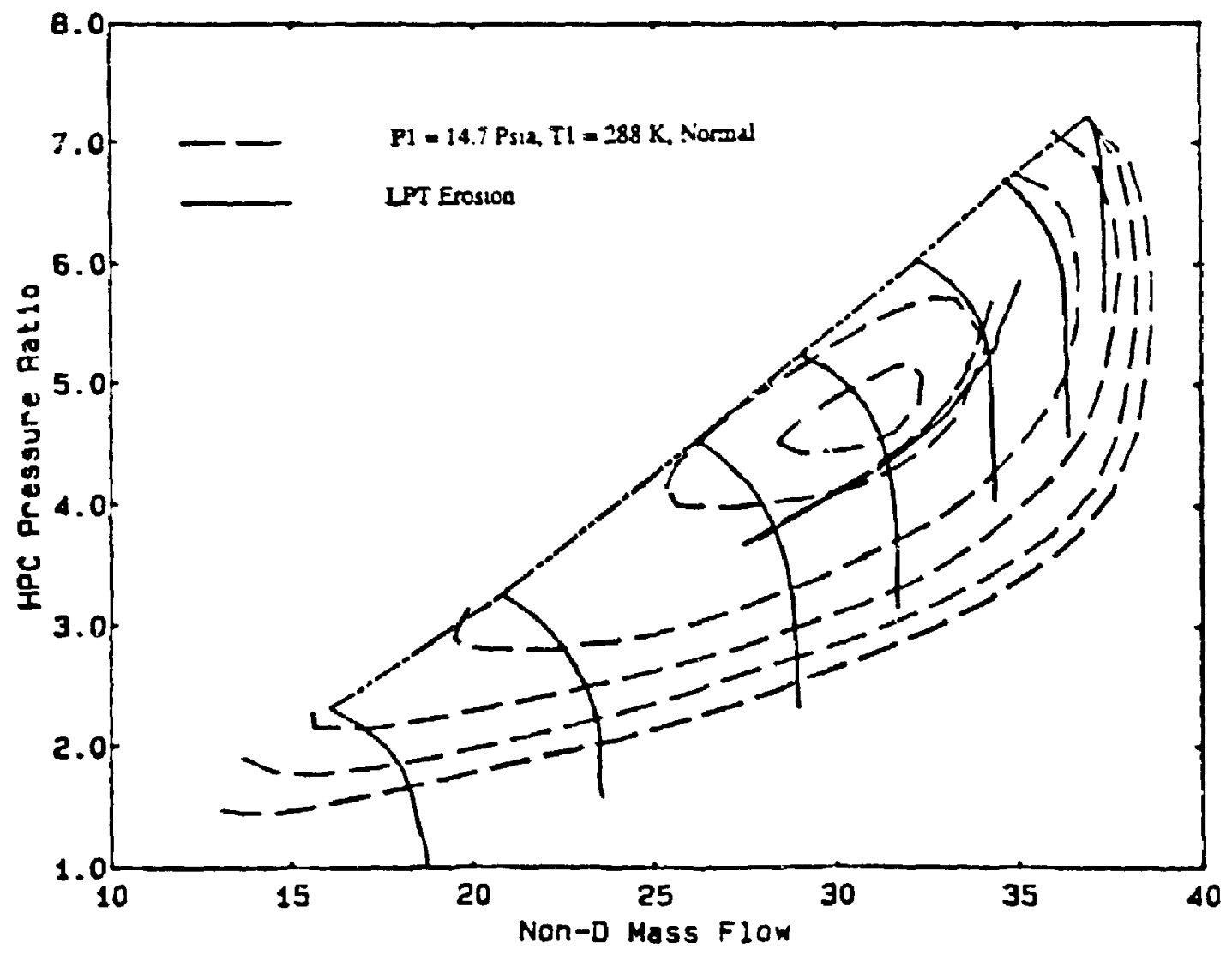

Fig. 44 (5) Effect of LPT Erosion on HPC Running Line 


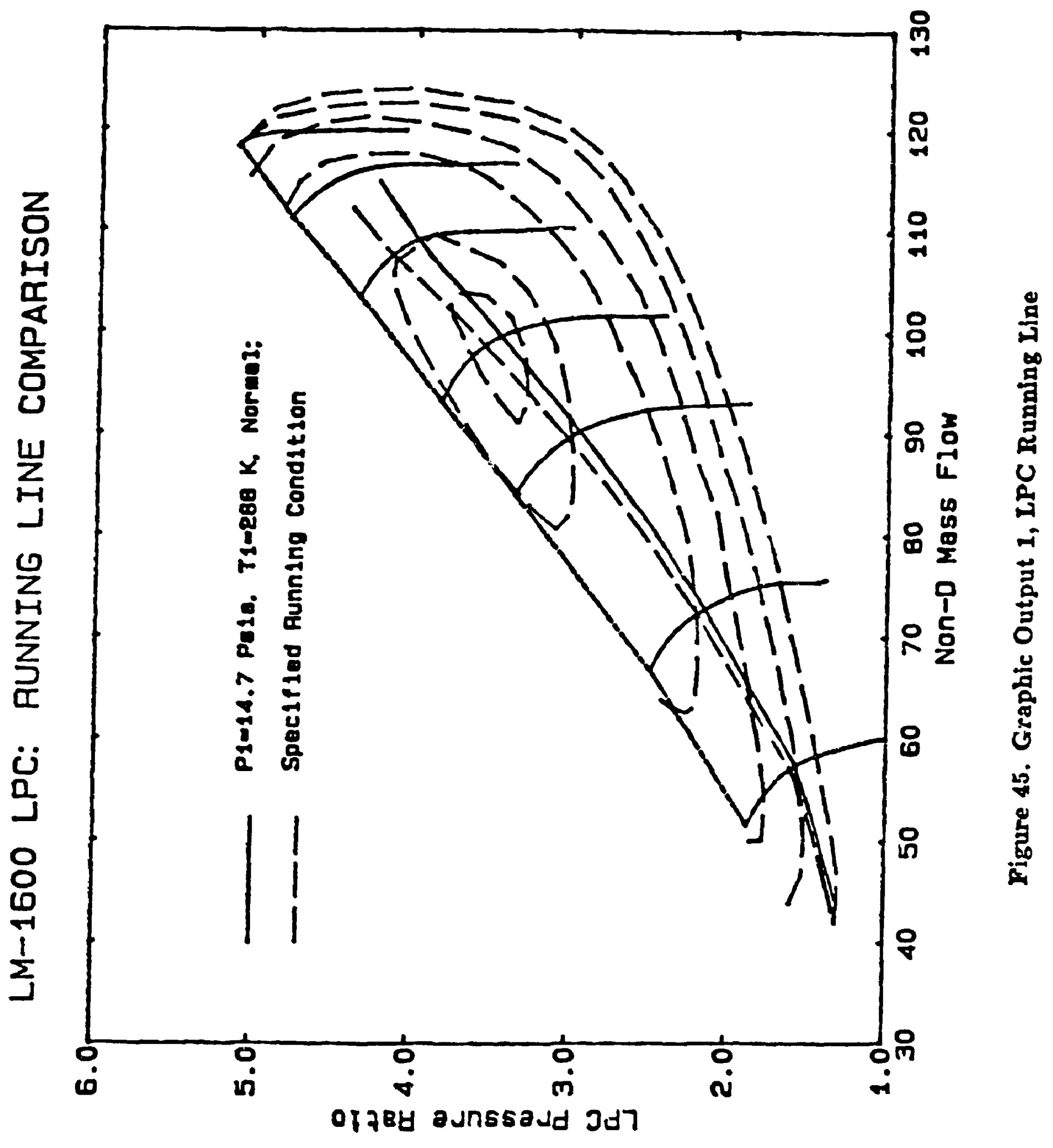




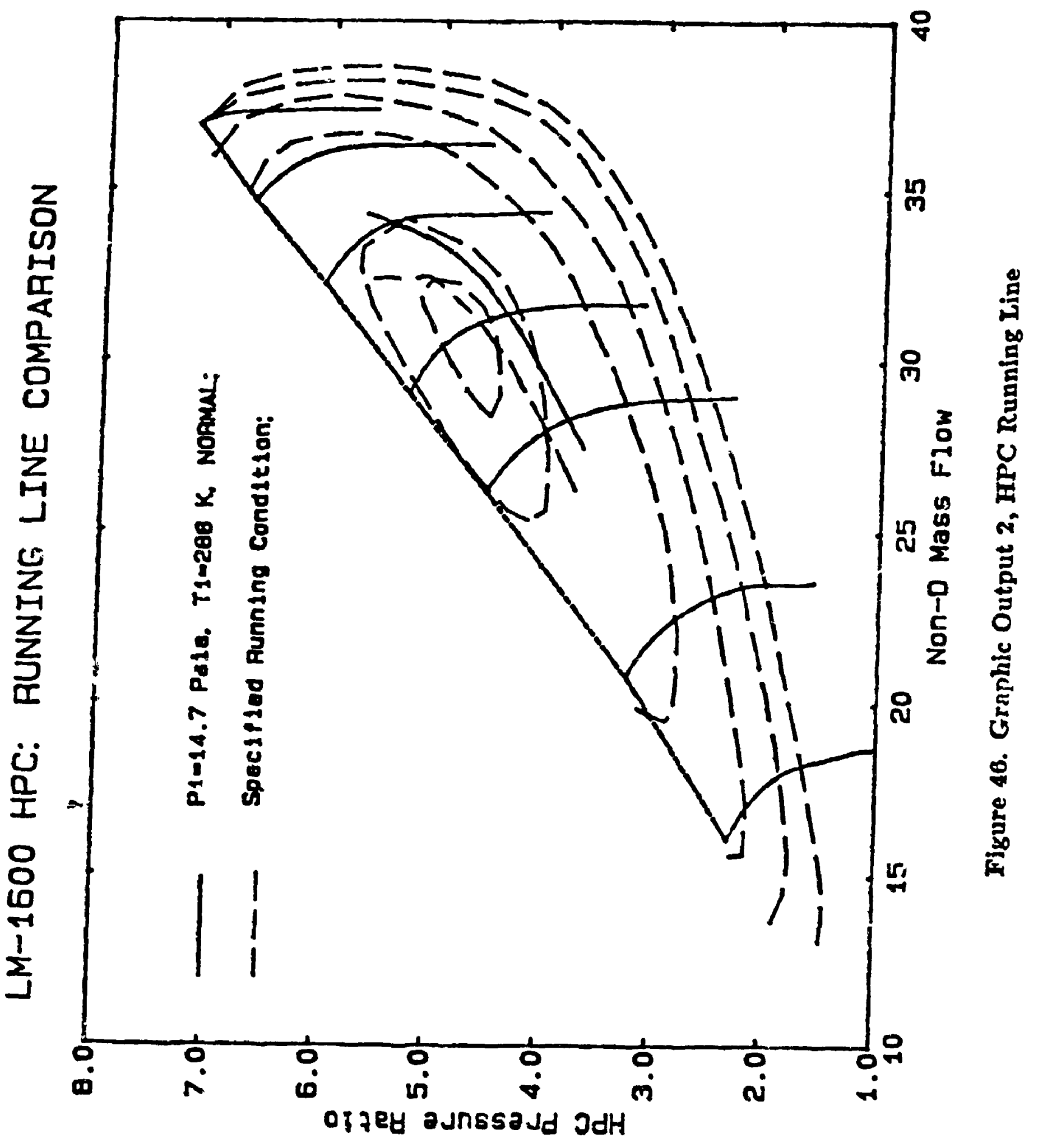




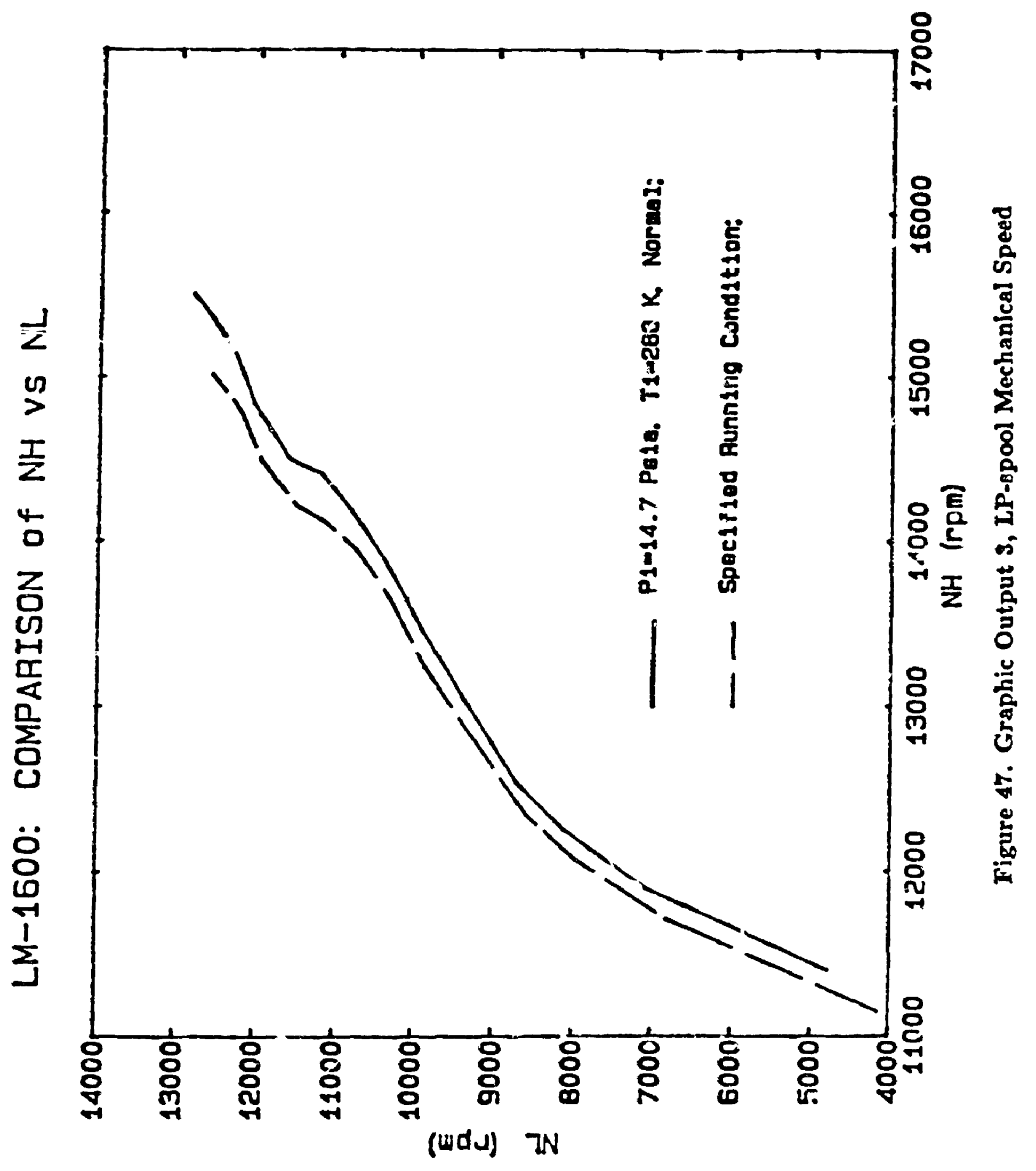



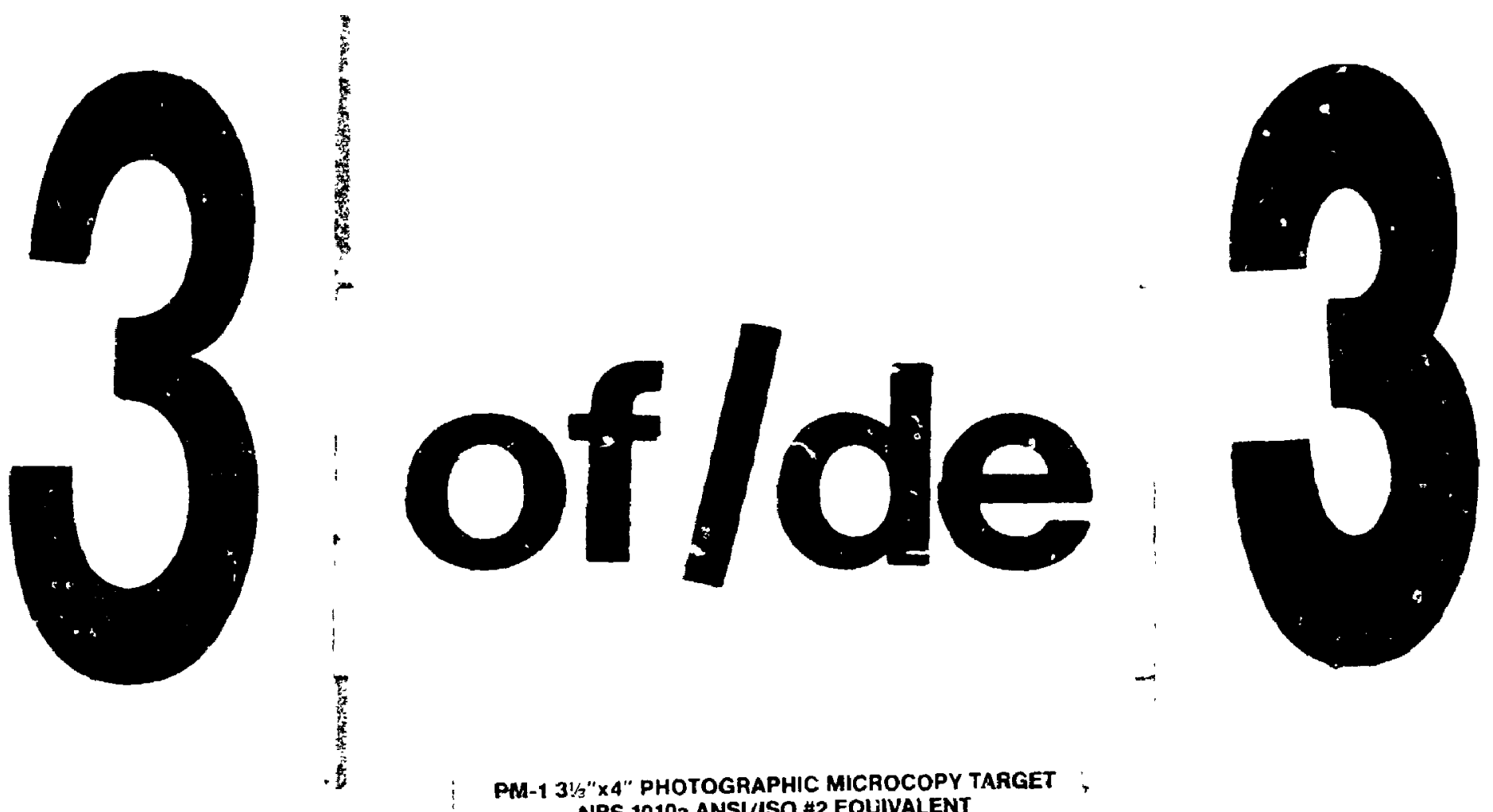

PM-1 31/3" $\times 4 "$ PHOTOGRAPHIC MICROCOPY TARGET ; NBS 1010a ANSI/ISO \#2 EQUIVALENT

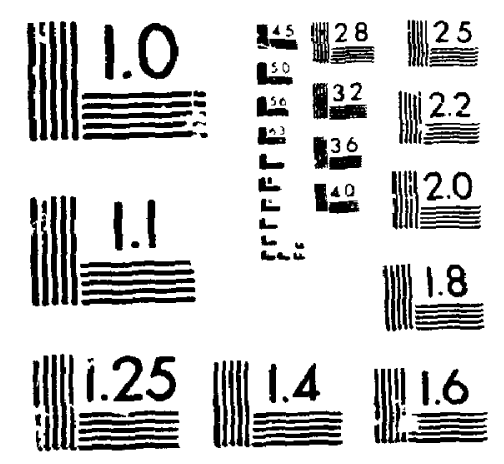




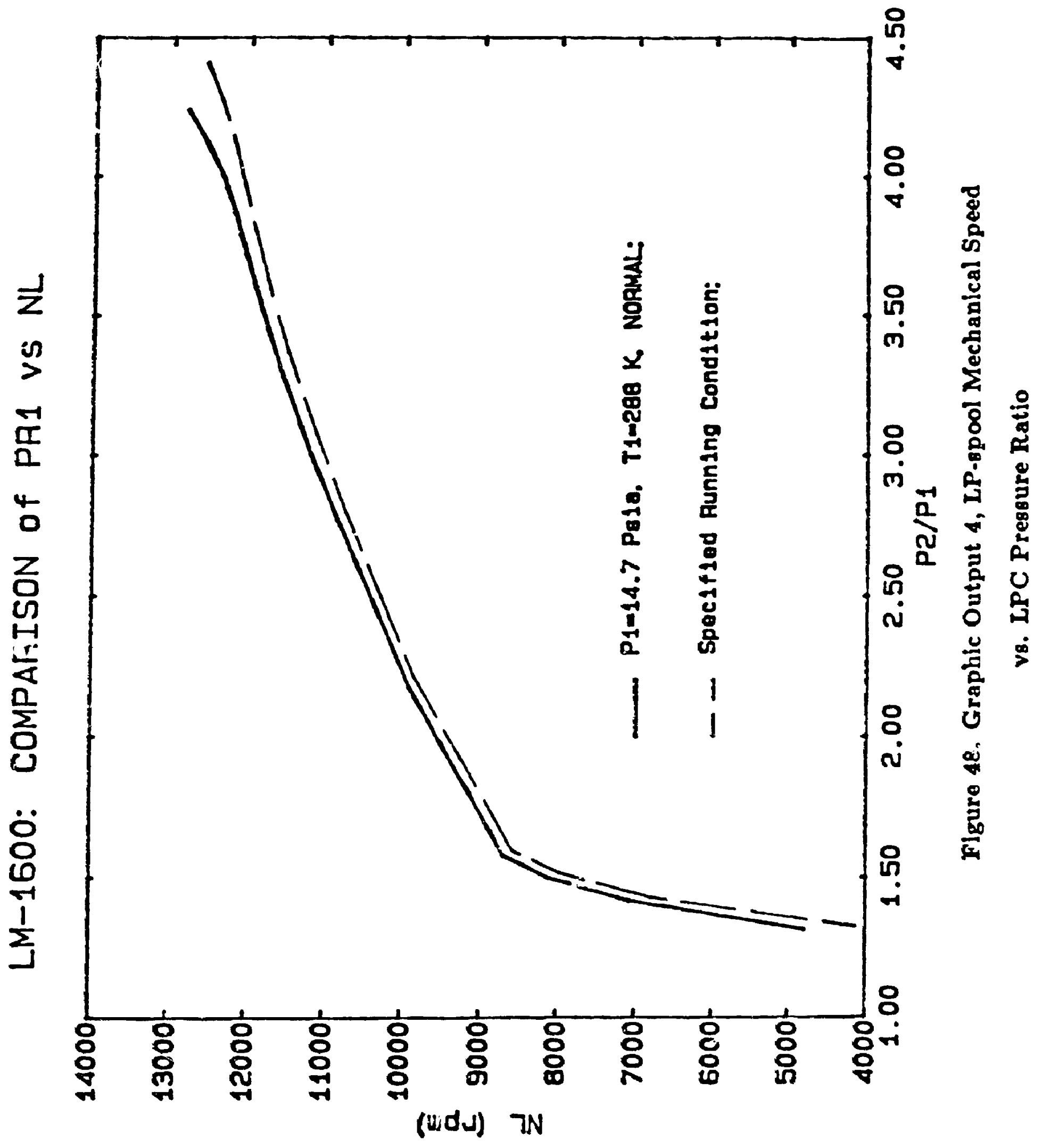




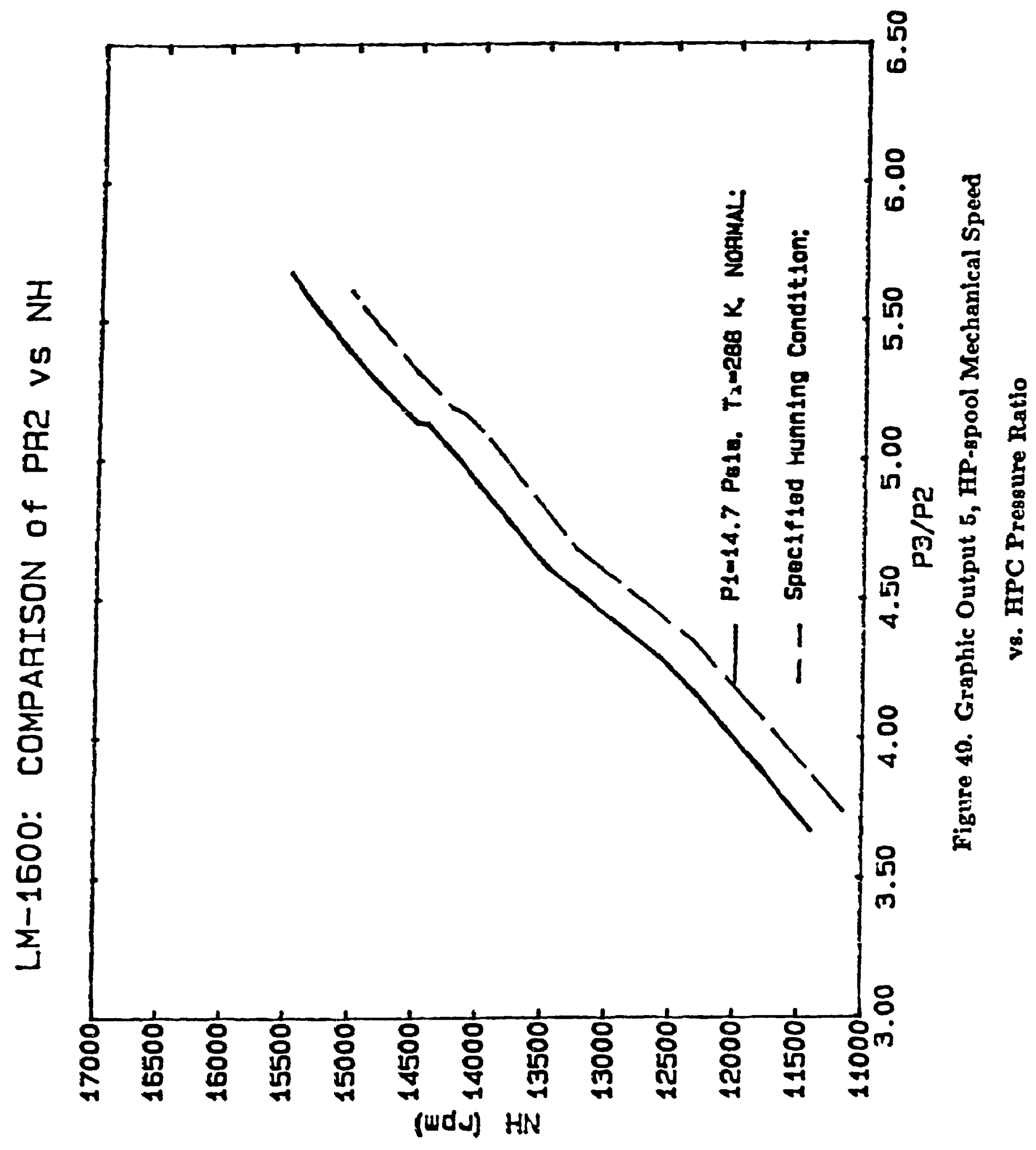




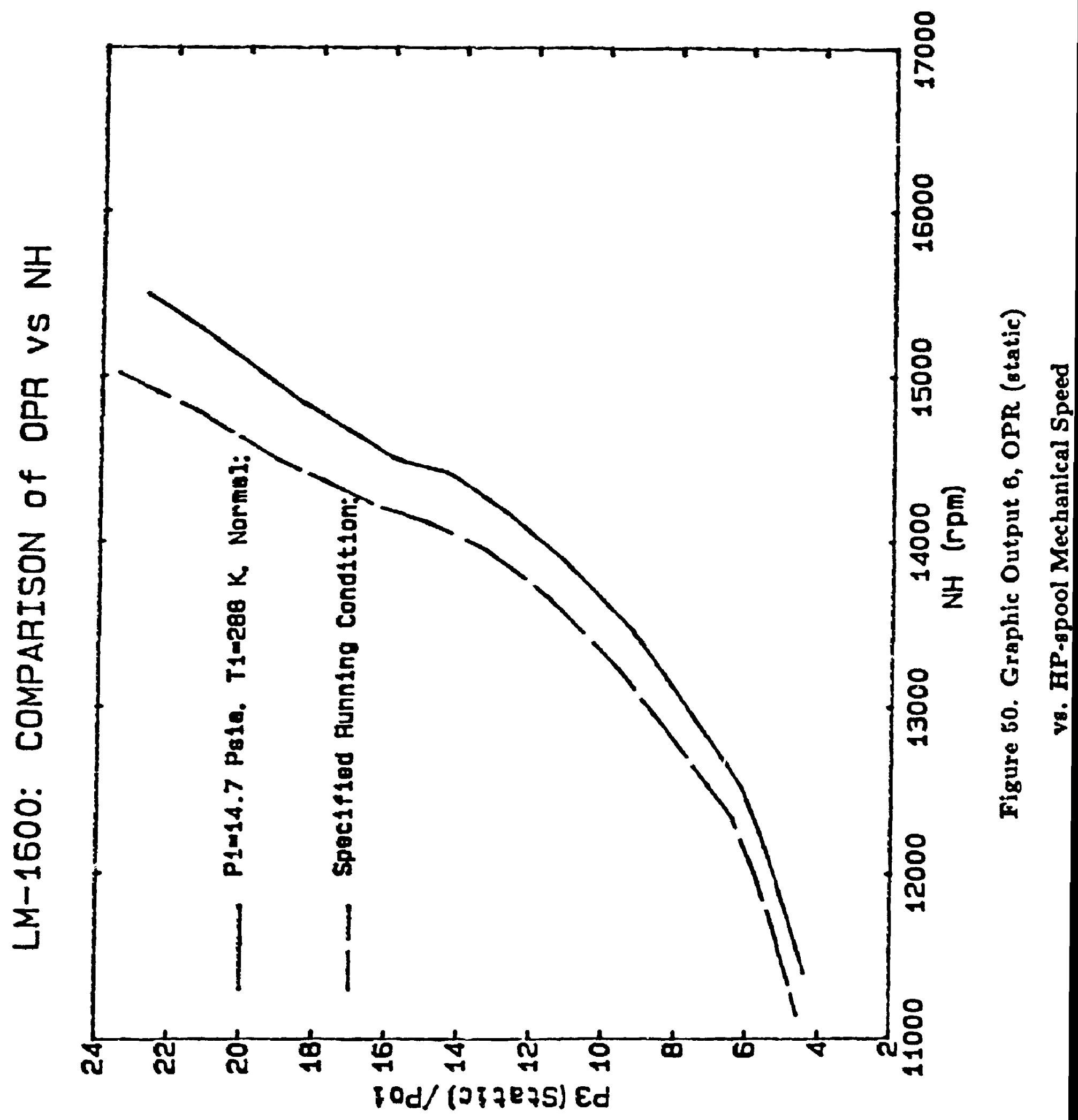




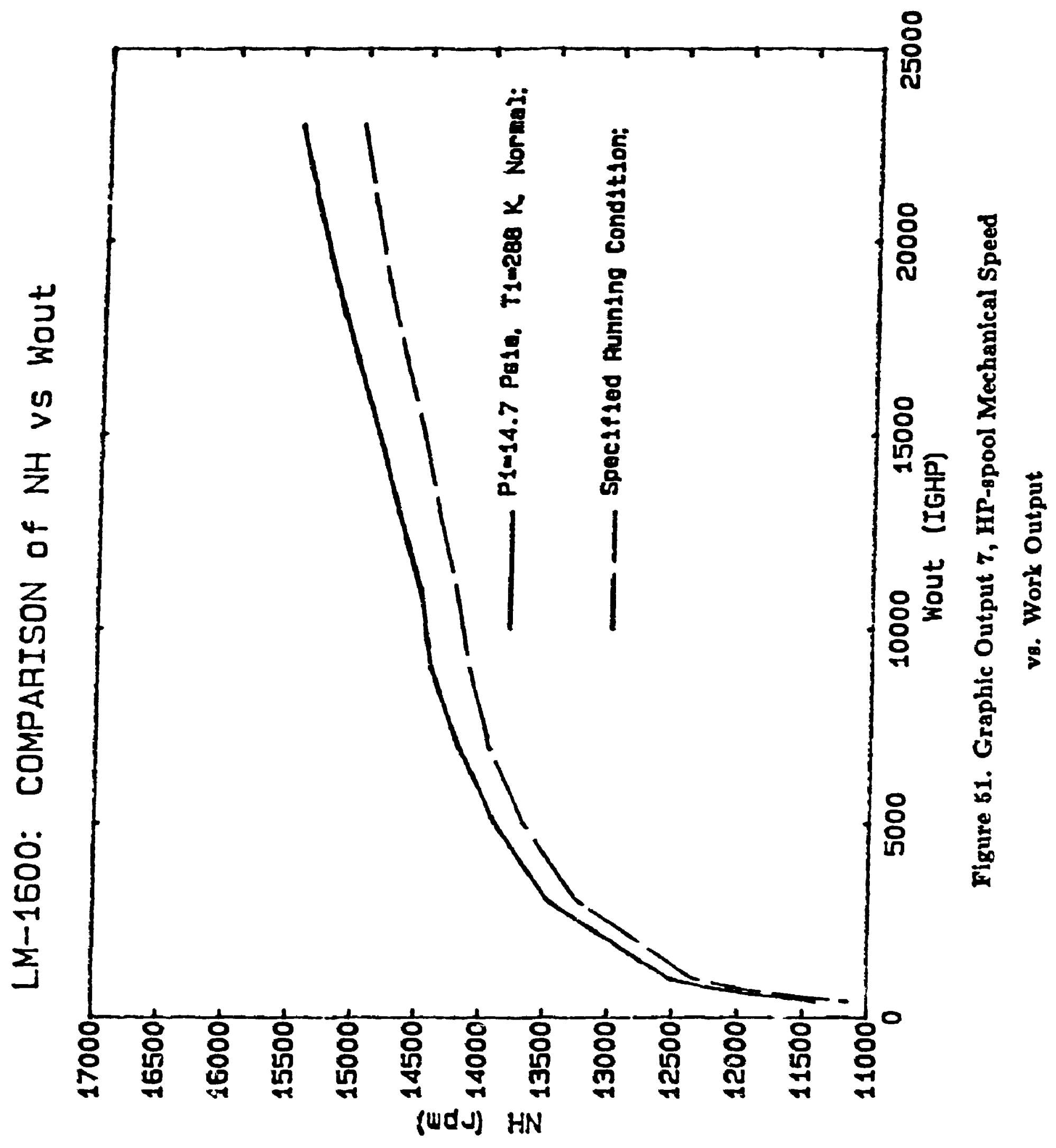




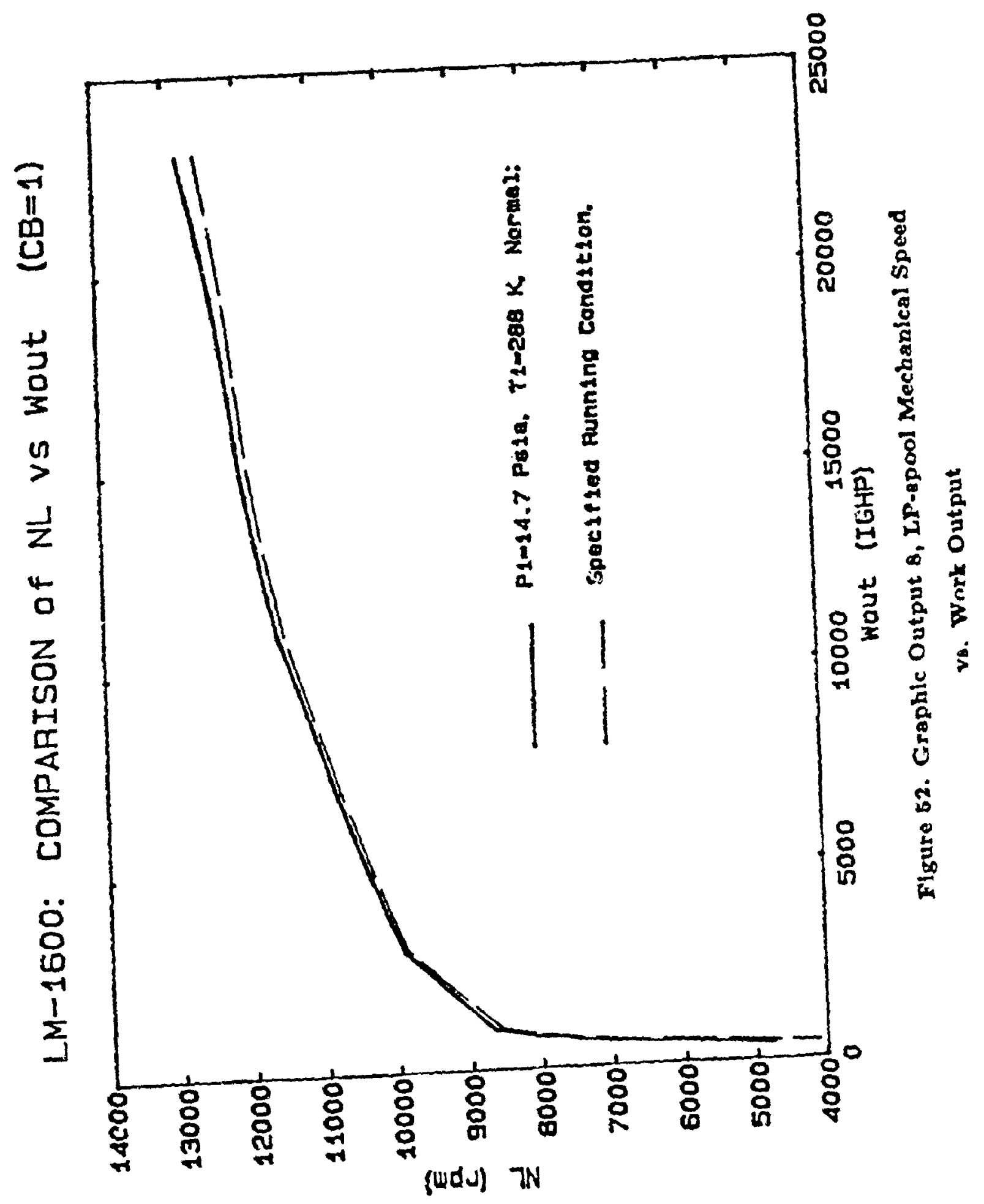




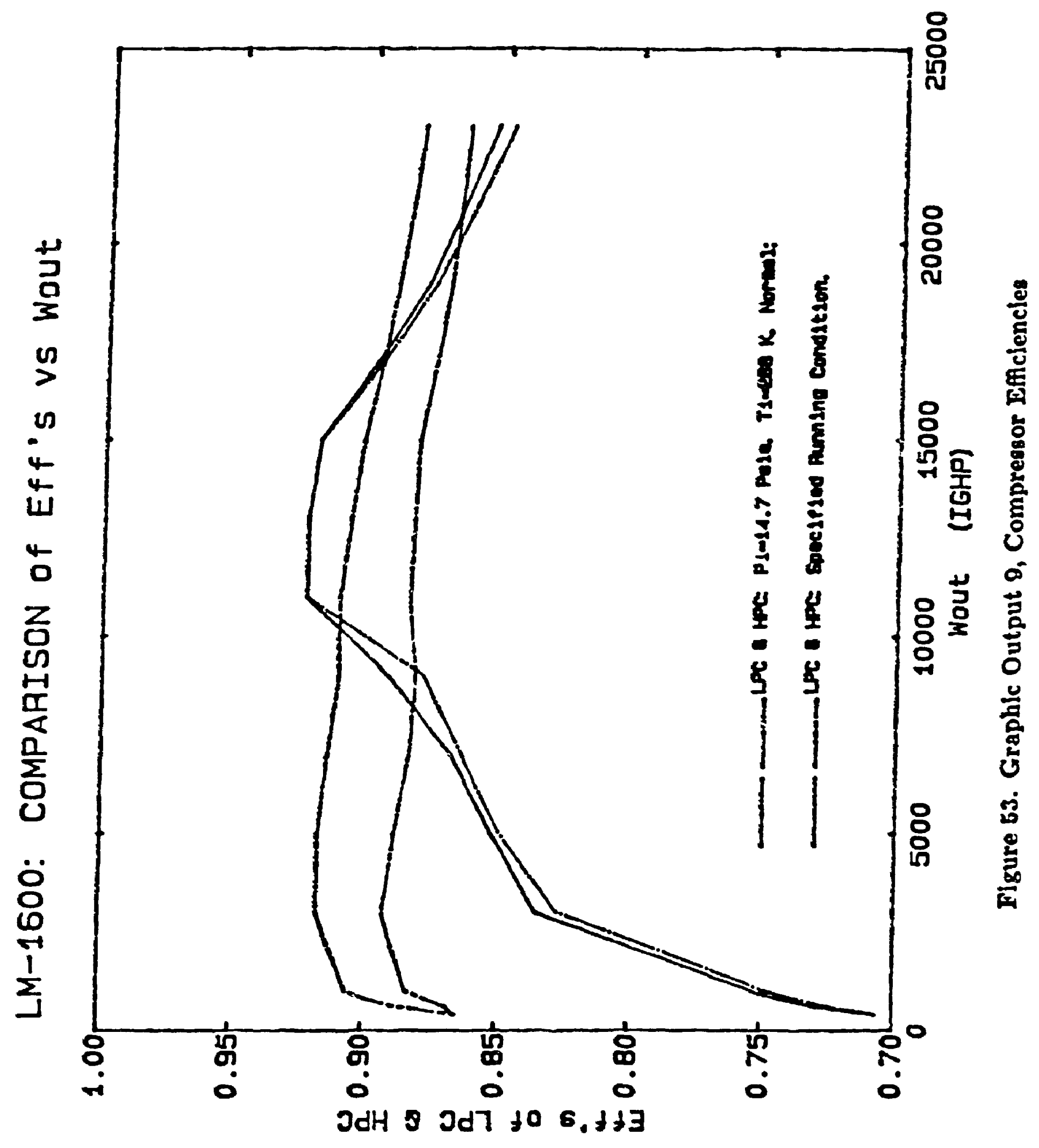




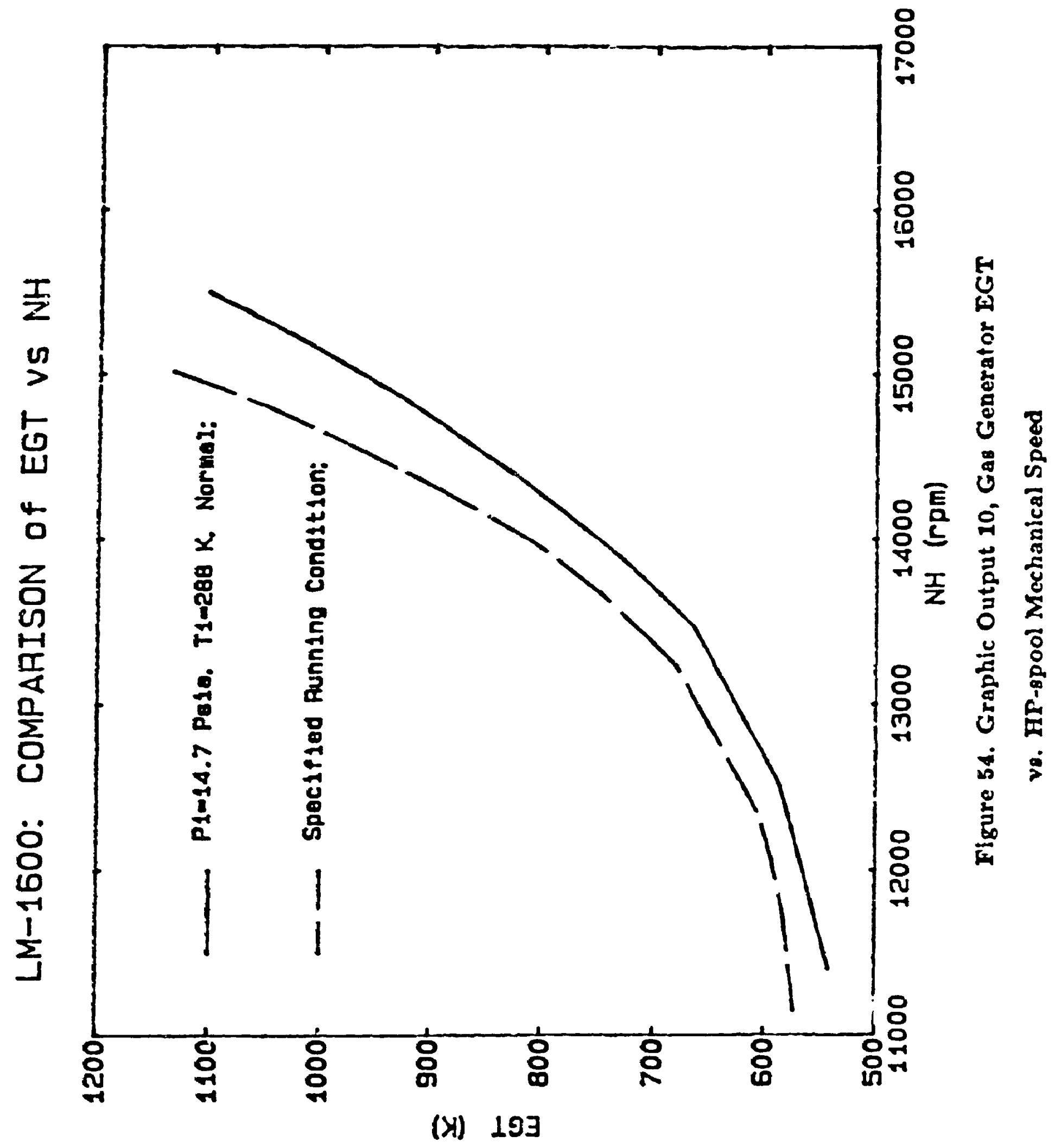




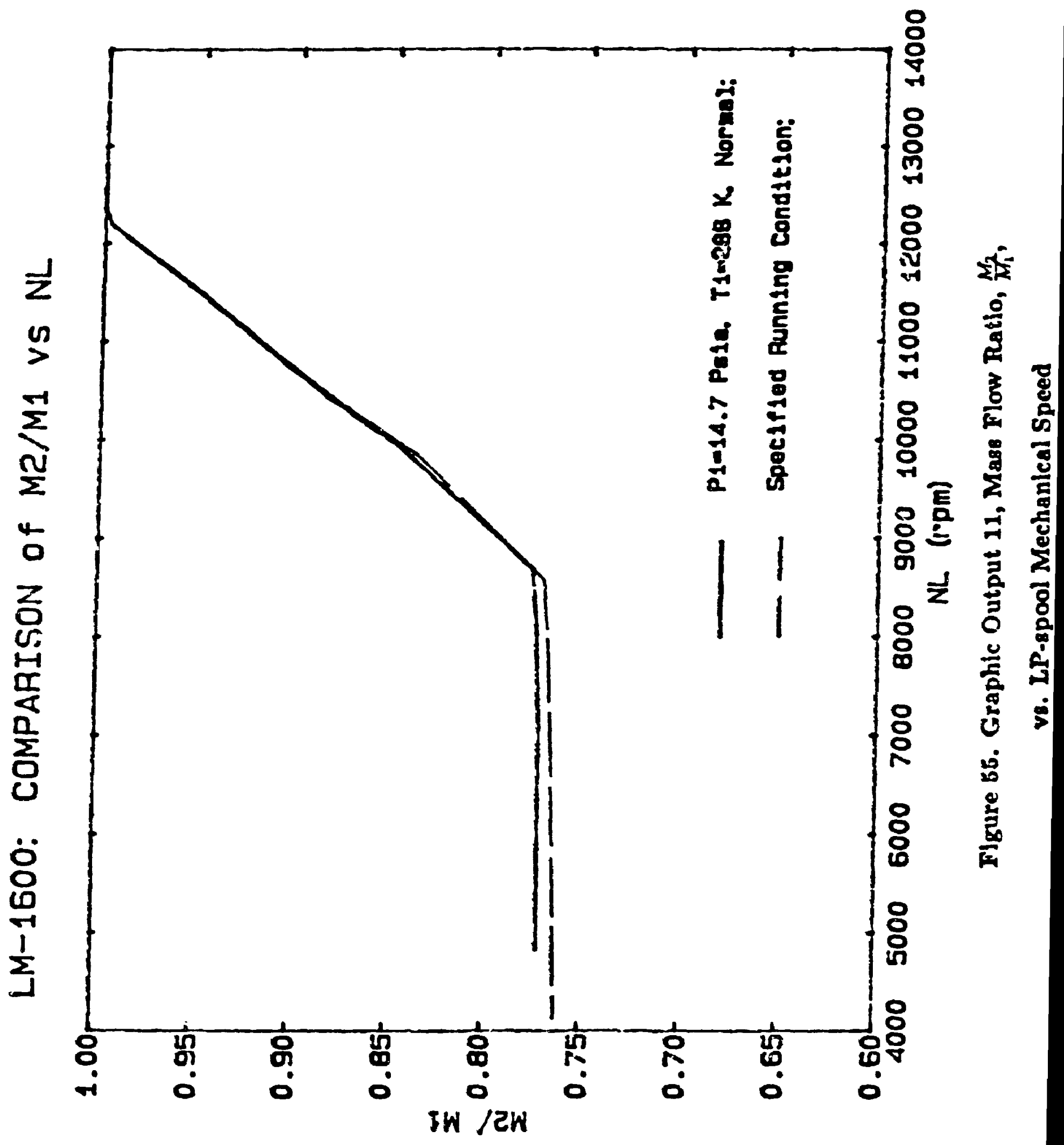




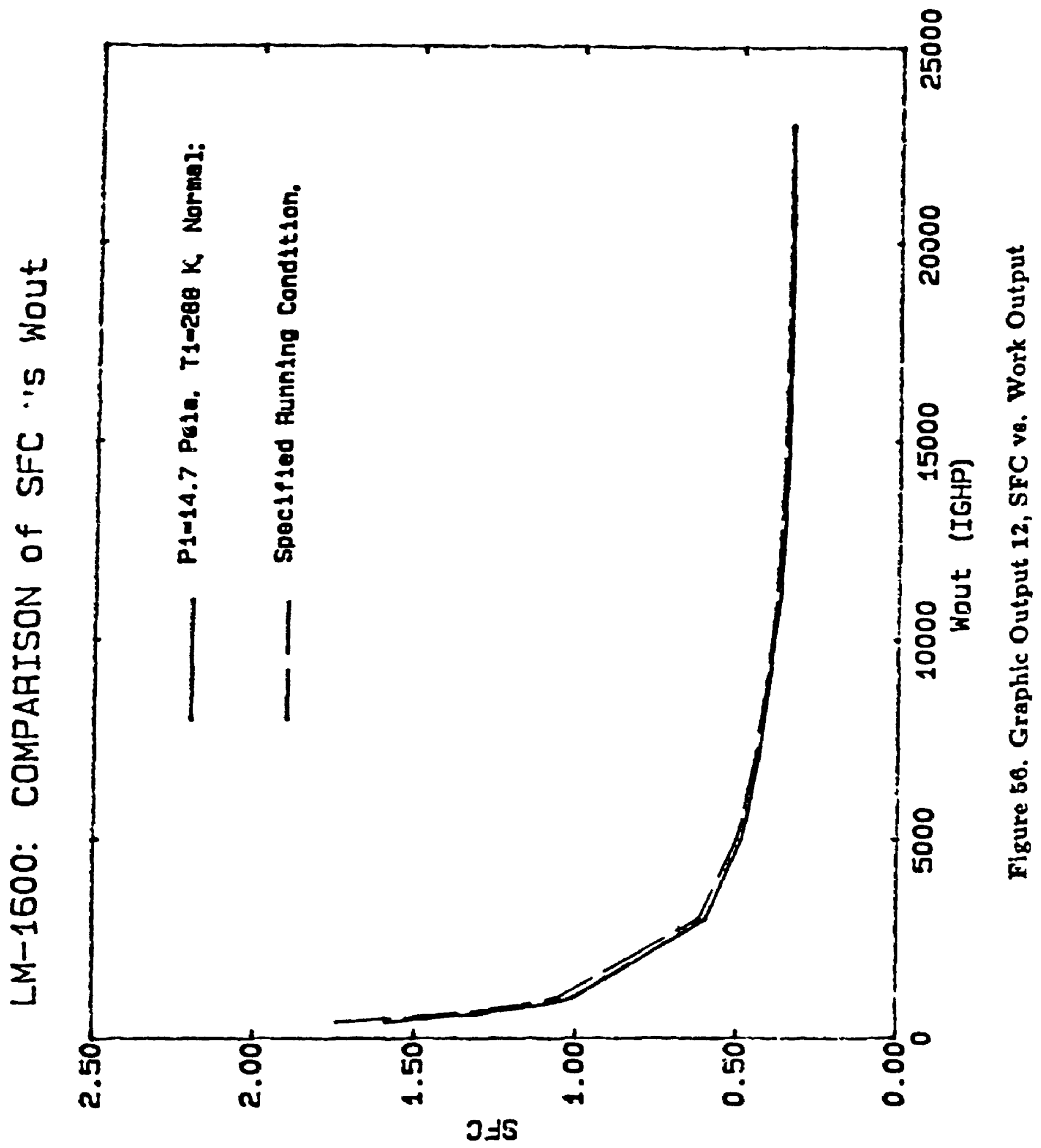



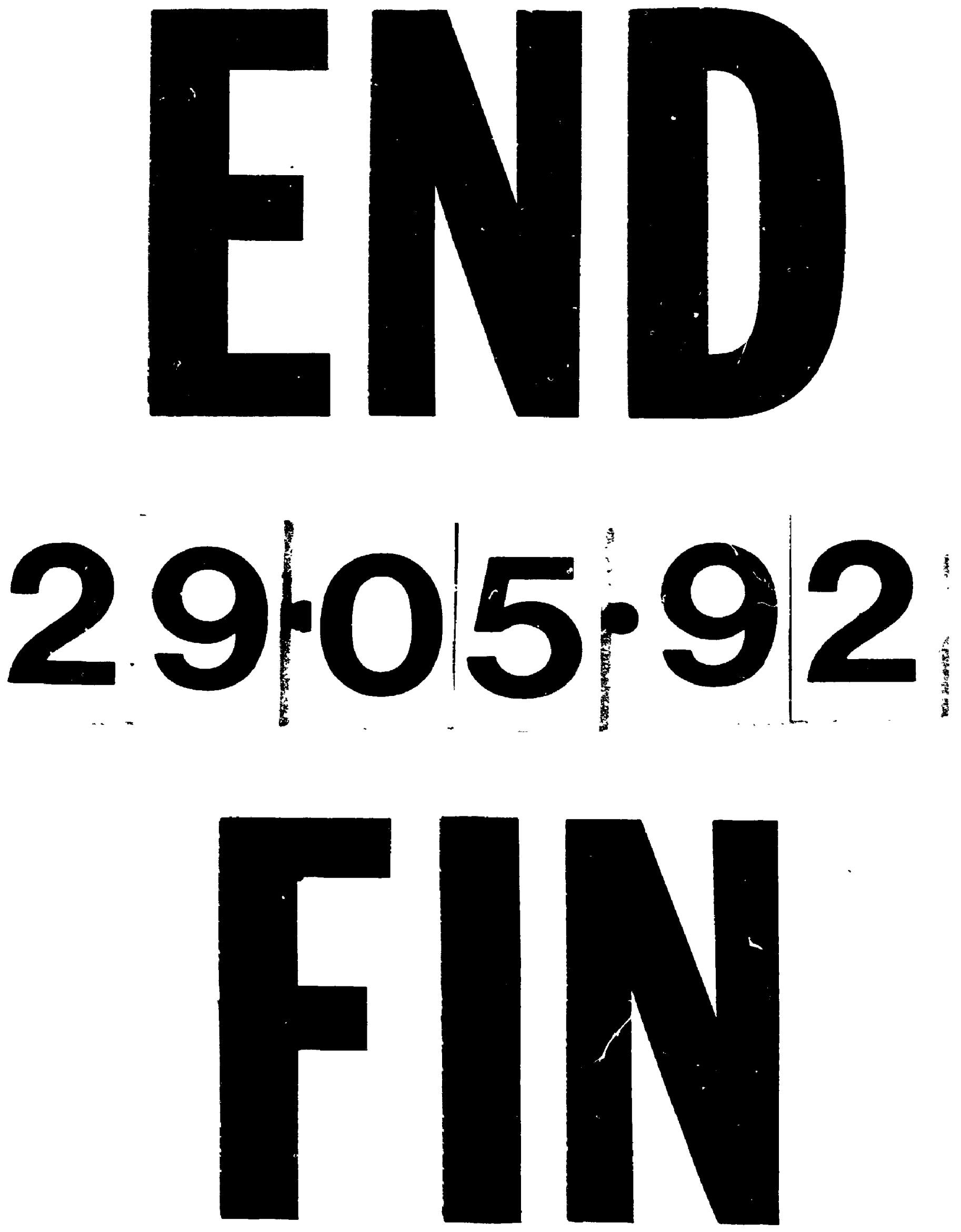